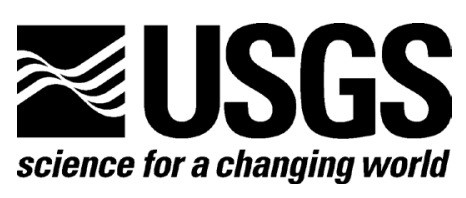

\title{
HAWAIIAN VOLCANO OBSERVATORY 1984 Annual Administrative Report
}

INTRODUCTORY NOTE BY THOMAS L. WRIGHT AND JENNIFER S. NAKATA

COMPILED BY JENNIFER S. NAKATA

\author{
SUMMARY 84 \\ SEISMIC DATA, JANUARY TO DECEMBER 1984 \\ BY JENNIFER S. NAKATA, ROBERT Y. KOYANAgi, \\ Alvin H. TOMORI, AND WilfREd R. TANigawa \\ CHRONOLOGICAL SUMMARY \\ BY THOMAS L. WRIGHT
}

OPEN-FILE REPORT 2007-1344

U.S. DEPARTMENT OF THE INTERIOR

U.S. GEOLOGICAL SURVEY 


\section{U.S. Department of the Interior DIRK KEMPTHORNE, Secretary \\ U.S. Geological Survey Mark D. Myers, Director}

\section{U.S. Geological Survey, Reston, Virginia 2007}

For product and ordering information:

World Wide Web: http://www.usgs.gov/pubprod

Telephone: 1-888-ASK-USGS

For more information on the USGS - the Federal source for science about the Earth, its natural and living resources, natural hazards, and the environment: World Wide Web: http://www.usgs.gov

Telephone: 1-888-ASK-USGS

Any use of trade, product, or firm names is for descriptive purposes only and does not imply endorsement by the U.S. Government.

Although this report is in the public domain, permission must be secured from the individual copyright owners to reproduce any copyrighted material contained within this report. 


\section{INTRODUCTORY NOTE}

The Hawaiian Volcano Observatory Summaries have been published in the current format since 1956. The Quarterly Summaries (1956 through 1973) and the Annual Summaries (1974 through 1985) were originally published as Administrative Reports. These reports have been compiled and published as U.S. Geological Survey Open-File Reports. The quarterly reports have been combined and published as one annual summary. All the summaries from 1956 to the present are now available as .pdf files at http://www.usgs.gov/pubprod.

The earthquake summary data are presented as a listing of origin time, depth, magnitude, and other location parameters. Network instrumentation, field station sites, and location algorithms are described. Tilt and other deformation data are included until Summary 77, January to December 1977. From 1978, the seismic and deformation data are published separately, due to differing schedules of data reduction.

There are eight quarters - from the fourth quarter of 1959 to the third quarter of 1961 — that were never published. Two of these ( $4^{\text {th }}$ quarter $1959,1^{\text {st }}$ quarter 1960) have now been published, using handwritten notes of Jerry Eaton (HVO seismologist at the time) and his colleagues. The seismic records for the remaining six summaries went back to California in 1961 with Jerry Eaton. Other responsibilities intervened, and the seismic summaries were never prepared.

\section{Chronology}

The following Kìlauea eruption chronology covers the two recent reports and the six missing quarters:

\begin{tabular}{|l|l|l|l|}
\hline Location & Beginning Date & Ending Date & Comment \\
\hline Kìlauea Iki crater (Kîlauea's summit) & $11 / 14 / 1959$ & $12 / 20 / 1959$ & 19 eruptive episodes \\
\hline Kapoho (lower east rift zone) & $1 / 13 / 1960$ & $2 / 18 / 1960$ & 4 eruption stages \\
\hline Halemaumau (Kīlauea's summit) & $2 / 24 / 1961$ & $2 / 24 / 1961$ & $\begin{array}{l}\text { Intermittent activity during } \\
\text { uninterrupted inflation fol- } \\
\text { lowing the 1960 eruption }\end{array}$ \\
\hline Halemaumau (Kīlauea's summit) & $3 / 22 / 1961$ & $3 / 25 / 1961$ & Same as above. \\
\hline Halemaumau (Kīlauea's summit) & $7 / 10 / 1961$ & $7 / 17 / 1961$ & Same as above. \\
\hline Heiheiahulu (middle east rift zone) & $9 / 22 / 1961$ & $9 / 25 / 1961$ & $\begin{array}{l}\text { First historical east rift erup- } \\
\text { tion at this location }\end{array}$ \\
\hline
\end{tabular}

The 1959-1960 eruptions were among two of the most spectacular Kilauea eruptions. The HVO staff was kept busy with acquisition of unusually high quantities of instrumental data and observations of the two sequences, which were separated by less than one month. Even with a year's interval before the beginning of the summit-east rift sequence in 1961, the staff never caught up, and the seismic records were set aside for later study.

A total of 1,672 earthquakes-1,106 for 1960 and 566 for 1961-are part of HVO's cataloged database. The annual listings have been appended to the $1^{\text {st }}$ Quarter Report of 1960 and to the $4^{\text {th }}$ Quarter Report for 1961 . The number of earthquakes is probably low, biased toward the larger magnitudes. The entire HVO catalog, including 1960 and 1961, is accessible from the ANSS CATALOG SEARCH site at http://www.ncedc.org/anss/catalog-search. 
UNITED STATES DEPARTMENT OF THE INTERIOR

GEOLOGICAL SURVEY

HAWAIIAN VOLCANO OBSERVATORY

SUMMARY 84

SEISMIC DATA, JANUARY TO DECEMBER 1984

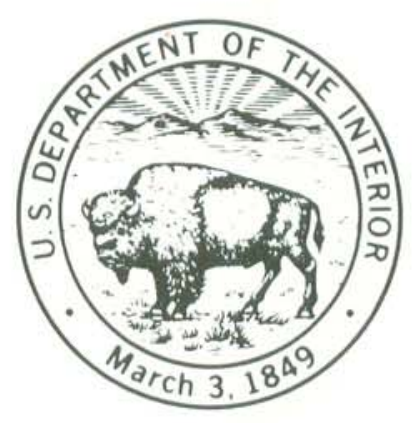

This report (map) is preliminary and has not been reviewed for conformity with U.S.Geological Survey editorial standards (and stratigraphic nomenclature). Any use of trade names is for descriptive purposes only and does not imply endorsement by the U.S.G.S.

Menlo Park, California 


\author{
UNITED STATES \\ DEPARTMENT OF THE INTERIOR \\ GEOLOGICAL SURVEY \\ HAWAIIAN VOLCANO OBSERVATORY \\ SUMMARY 84 \\ SEISMIC DATA, JANUARY TO DECEMBER 1984
}

BY

JENNIFER S. NAKATA, ROBERT Y. KOYANAGI, ALVIN H. TOMORI, WILFRED R. TANIGAWA CHRONOLOGICAL SUMMARY

BY

THOMAS L. WRIGHT

\title{
OBSERVATORY STAFF
}

\section{GEOLOGY}

TONI D. ABADIE * C. CHRISTINA HELIKER + JOHN R. HOFFMANN + GEORGE E. ULRICH + THOMAS L. WRIGHT + (SCIENTIST-IN-CHARGE)

GEOPHYSICS

CRAIG C. CRISSINGER ROBERT W. DECKER * (SCIENTIST-IN-CHARGE)

JOHN J. DVORAK KENNETH T. HONMA GARY Y. HONZAKI DALLAS B. JACKSON GEORGE KOJIMA ROBERT Y. KOYANAGI ALLAN J. LARGO JENNIFER S. NAKATA ARNOLD T. OKAMURA GARY S. PUNIWAI WILFRED R. TANIGAWA ALVIN H. TOMORI

\section{GEOCHEMISTRY}

L. PAUL GREENLAND

REGINALD T. OKAMURA (CHIEF OF OPERATIONS)

J. B. STOKES
SUPPORT

T. JANE DIXON

THOMAS T. ENGLISH

DOROTHY C. FOOTER

JOHN C. FORBES

J. D. GRIGGS

RONALD Y. HANATANI

MARIAN M. KAGIMOTO

MAURICE K. SAKO

PAULINE N. TAMURA

AKIRA YAMAMOTO

KENNETH M. YAMASHITA *

JOAN M. YOSHIOKA

\section{AFFILIATES}

JANE BUCHANAN-BANKS (GEOLOGIST)

NORMAN B. BANKS (GEOLOGIST)

JAMES KAUAHIKAWA (GEOPHYSICIST)

JOHN P. LOCKWOOD (GEOLOGIST)

RICHARD B. MOORE (GEOLOGIST)

CHRISTINA A. NEAL (GEOLOGIST)

MARJORIE SUMMERS

VICKI B. TAYLOR

FRANK A. TRUSDELL

EDWARD W. WOLFE (GEOLOGIST)

+ Arrived during 1984
* Left during 1984 


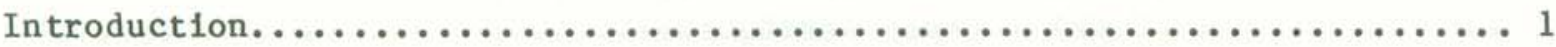

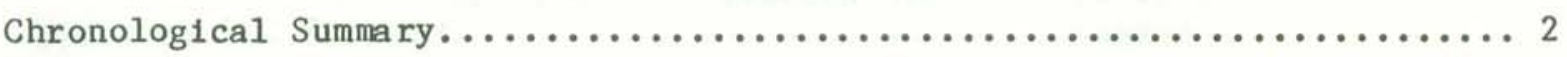

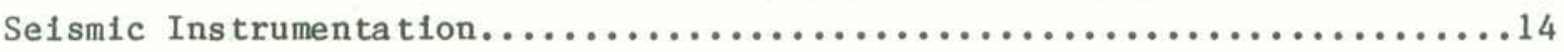

Figure 1 Map of Hawa11 showing geographic and geologic

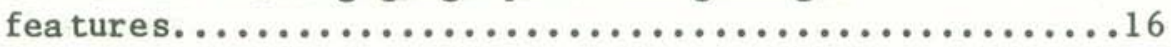

Figure 2 Map of Hawail showing active seismic stations.............

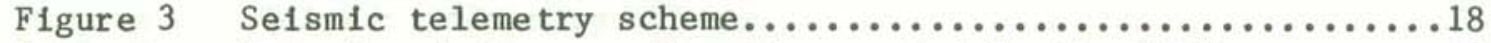

Table 1 Seismic stations on Hawail operated by the USGS.........19

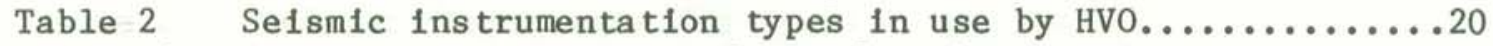

Figure 4 System response curve of the four basic seismo-

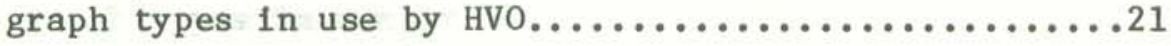

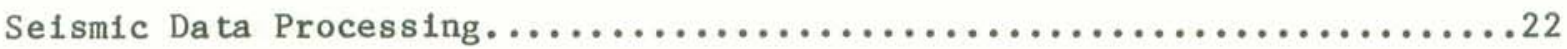

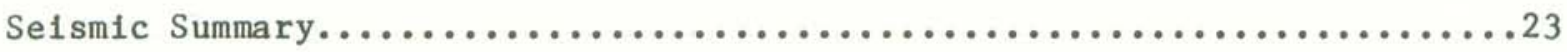

Table 3 Number of earthquakes and minutes of tremor recorded on seismographs around Kilauea

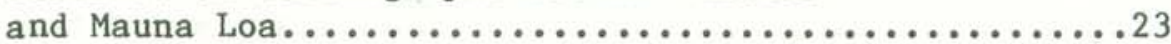

Table 4 Coordinates of named regions used for classifying

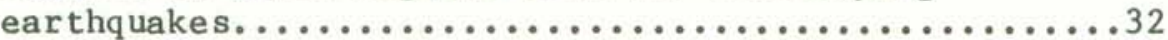

Figure 5 Earthquake classification map, Kilauea, depths $0-5 \mathrm{~km} . \ldots 34$

Figure 6 Earthquake classification map, Kilauea, depths 5-13 km...35

Figure 7 Earthquake classification map, Hawa11, depths $0-13 \mathrm{~km} . \ldots 36$

Figure 8 Earthquake classification map, Hawa11, depths 13-99 km....37

Figure 9 Hawalian Islands Earthquake Locations, depths 0-60 km,

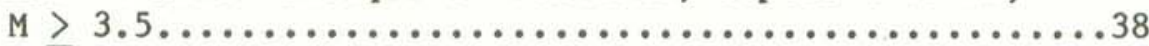

Figure 10 Hawa11 Island Earthquake Locations, depths $0-5 \mathrm{~km}$,

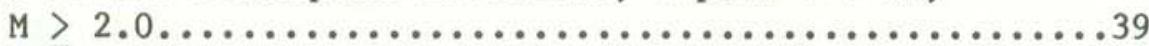

Figure 11 Hawai1 Island Earthquake Locations, depths $5.1-13 \mathrm{~km}$,

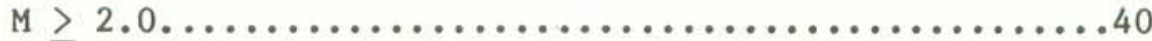

Figure 12 Hawa11 Island Earthquake Locations, depths 13.1-60 km,

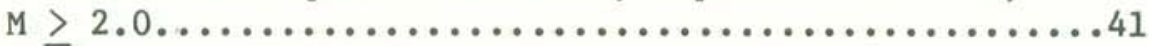

Figure 13 Kilauea Summit Earthquake Locations, depths 0-5 km,

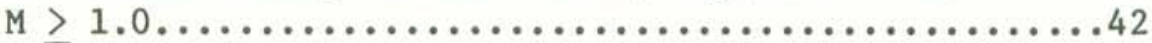

Figure 14 Kilauea Summit Earthquake Locations, depths 5.1-13 km,

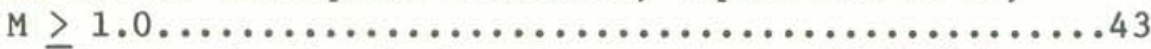

Figure 15 Kilauea Summit Earthquake Locations, depths $13.1-60 \mathrm{~km}$,

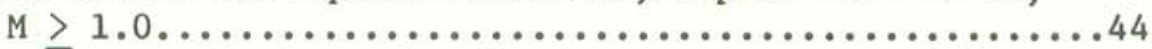

Figure 16 Kilauea South Flank Earthquake Locations, depths $0-5 \mathrm{~km}$,

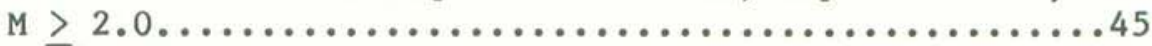

Figure 17 Kilauea South Flank Earthquake Locations, depths 5.1-13

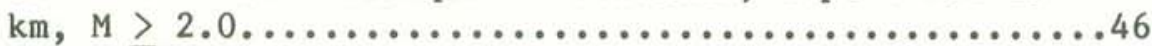

Figure 18 Kilauea South Flank Earthquake Locations, depths 13.1-60

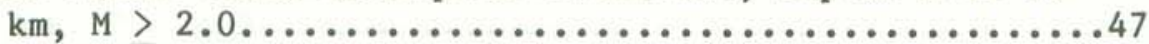

Figure 19 Mauna Loa Sümit Earthquake Locations, depths $0-5 \mathrm{~km}$,

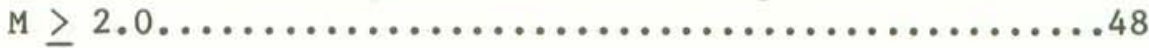


Figure 20 Mauna Loa Summit Earthquake Locations, depths $5.1-13 \mathrm{~km}$,

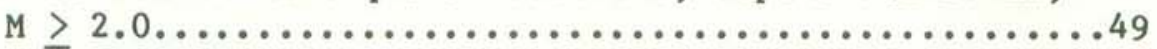

Figure 21 Mauna Loa Summit Earthquake Locations, depths 13.1-60

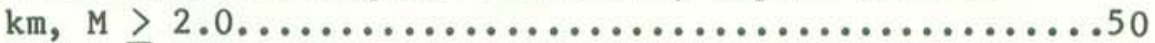

Figure 22 Hawail Island Earthquake Locations, depths 0-60 km,

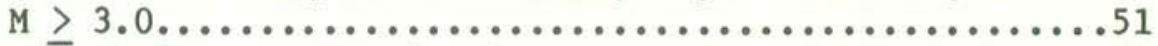

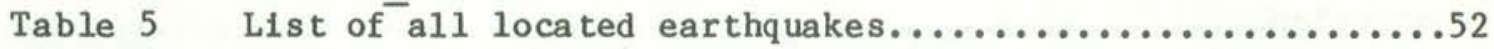

Table 6 List of located earthquakes of magnitude 3.0 or

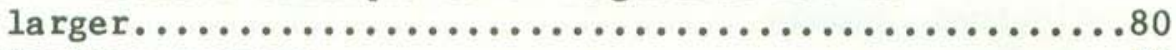

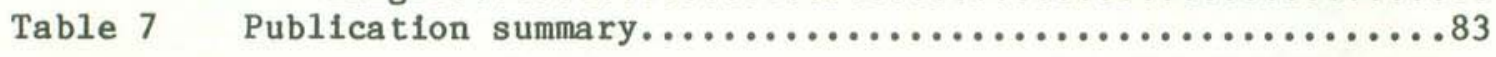




\section{INTRODUCTION}

The Hawalian Volcano Observatory (HVO) summary presents data gathered during the year together with a chronological narrative describing the volcanic events and significant observatory related activities. The seismic summary is offered without interpretation as a source of preliminary data. The seismic summary is complete in the sense that all data routinely gathered by the observatory are included. The emphasis in collection of tilt and deformation data has shifted from quarterly measurements at a few water-tube tilt stations ("wet" tilt) to a larger number of continuously recording borehole tiltmeters, repeated measurements at numerous spirit-level tilt stations ("dry" tilt), and surveying of level and trilateration networks. Because of the large quantity of deformation data now gathered and differing schedules of data reduction, the seismic and deformation summaries will be published separately.

The HVO summaries have been published in various formats since 1956. Summaries prior to 1974 were issued quarterly, but cost, convenience of preparation and distribution, and the large quantities of data dictated an annual format beginning with summary 74 for the year 1974. Summary 74 includes an extensive description of the seismic instrumentation, calibration and processing used in recent years. The present summary includes enough background information on the seismic network and processing to use the data and understand the essentials of how 1 t was gathered.

A report tabulating the instrumentation, calibration and recording history of each seismic station in the network by Klein and Koyanagi is available as a USGS open file report ("Hawailan Volcano Observatory Seismic Network History 1950-79," U.S.G.S. open file report 80-302, 1980). It is designed as a reference for users of seismograms and phase data, and so includes and expands the information in the station table in this summary. 
Chronolog1cal Summary - 1984

Events in 1984 featured a long-awalted eruption of Mauna Loa, continued monthly activity at Kilauea's Pu'u $0^{\prime} \mathrm{O}$ vent, approval and funding, after 8 years, for construction of a new HVO facility, and changes in staff, including the arrival of a new scientist-in-charge.

The Mauna Loa eruption, March 25-April 15, was clearly the high point of the year. A forecast published in 1976 correctly anticipated its location (northeast rift zone between 2800 and 3000 meters altitude) but missed the time by 6 years. Another forecast published in 1983 anticipating an eruption "during the next two years" proved correct. A summary description of the eruption is given by Lockwood and others (1985). Table C-1 summarizes significant data for the eruption and Figure C-1, taken from Lockwood and others (1985), shows the extent of flows. The eruption was notable for 1 ts well developed lava channel which delivered lava very efficiently to the lower slopes of the volcano (slightly below 1000 meters elevation) where it posed a threat to the city of Hilo (Fig. C-1). Hilo was spared, however, by a repeated breakdown of the main channel at an altitude of about $2000 \mathrm{~m}$. This process, a natural lava diversion, was observed and documented for the first time during this eruption. It appears that the diversion results from "aging" of the channel whereby an initially smooth channel surface characterized by laminar flow is increasingly disrupted by erosion of the enclosing levees. Pieces of the levee eventually become sufficlently large and/or numerous that channel blockage results, the flow overspills the levee, and the old channel is abandoned (see Lipman and Banks, in press). The chemistry of the Mauna Loa lavas was extremely uniform (Fig. C-2), and similar to that erupted in 1975, both being olivine-poor and slightly fractionated. Evidently the top of a much larger reservoir of magma was tapped. Temperatures of the lava remained constant, consistent with the absence of chemical change, but the crystallinity increased dramatically during the eruption, evidence that the lava was initially undercooled (Lipman and Banks, in press). Increase in viscosity associated with higher crystallinity may also have contributed to the aging of the lava channels (see above).

Deformation data were obtained for the first time to cover a complete deflation of Mauna Loa. A total of over 100 microradians of deflation was recorded at one site, which occurred in response to $63 \mathrm{~cm}$ of vertical subsidence measured by leveling surveys. A recording tiltmeter showed 40 microradians of rapid uplift in the hour before the outbreak, reflecting dike emplacement. Horizontal changes are also large but are complicated by a combination of expansion of certain lines in response to dike emplacement and contraction of all lines in response to removal of magma at depth. Modeling of the Mauna Loa inflation and deflation showed a magma storage area located south of the caldera at a depth of about $3 \mathrm{~km}$, very similar to the geometry of the Kilauea summit magma storage reservoir (Decker and others, 1983). This conclusion is consistent with similar sizes of the summit calderas of Kilauea and Mauna Loa, and demonstrates that the magma chambers of the Hawalian shields move up with the growth of the shield, retaining a constant relationship to the upper surface of the volcano (Ryan, in press).

Kilauea, in contrast to Mauna Loa's single eruption, was in eruption 16 different times from Pu'u $\mathrm{O}^{\prime} \mathrm{O}$ on the east rift zone for an aggregate eruption length of 15 days and an aggregate volume of $149 \times 10^{6} \mathrm{~m}^{3}$ (Table C-2; Fig. C-3; Fig. 
C-4). The average lava production for each volcano was similar, about $10^{7} \mathrm{~m}^{3}$ of lava per day of eruption. The chemical composition (Fig. C-2) was more uniform than that of the first year of eruption, attesting to a more open summit-rift connection. The $\mathrm{Pu}$ 'u $0^{\prime} \mathrm{o}$ cone grew from a height of $78 \mathrm{~m}$ at the end of 1983 to $175 \mathrm{~m}$ at the end of 1984. The lavas spread mainly northeast and southeast from $\mathrm{Pu} \mathrm{u}^{\prime} \mathrm{O}^{\prime} \mathrm{O}$ and threatened, but did not destroy, buildings in the Royal Gardens subdivision, which was hit several times by eruptions from Pu'u 0'o in 1983. The longest eruptive episode of 1984, episode 18, lasted 60 hours and sent a flow to within $1 \mathrm{~km}$ of the ocean (Fig. C-3). Three isolated dwellings were overrun near the terminus of the flow in the vicinity of Kapaahu. The previous episode (17) took place during the Mauna Loa eruption. Neither eruption had an obvious effect on the eruptive behavior of the other, or on the shallow seismicity and summit tilt patterns associated with the two eruptions. This confirmed the essential independence of the shallow plumbing of the two volcanoes, long inferred from the consistently different chemical composition of lavas from the two volcanoes (Fig. C-2).

Systematic gravity measurements were made on both volcanoes in 1984 (Johnson, in press). A summit monitor at Kilauea, comparing gravity at a stable base station with gravity at a station near the center of inflation-deflation, demonstrates that the gravity signal is dominated by mass changes which exceed the changes expected from uplift or subsidence alone (Johnson, in press). The interpretation suggests that magma filling the summit reservoir is compressed (becomes denser) as the reservolr fills and, conversely, expands (becomes less dense) as pressure is relieved during transport to the east rift zone. Gravity data also helps to reconcile the discrepency between horizontal and vertical changes found in modeling the Kllauea magma reservolr. Cross-caldera horizontal changes are always larger than expected from modeling of vertical changes and gravity data supports the idea that the summit reservoir is slowly expanding in the direction of the mobile south flank of the volcano. Apart from the short term changes in gravity, the long term gravity signature at Kilauea hints at continuing development of a Bouguer anomaly related most probably to solidification of intrusions at depth and accumulation of excess olivine in the lower parts of the storage complex. Gravity measurements at Mauna Loa show a somewhat different story. Gravity changes during the large deflation associated with the Mauna Loa eruption closely tracked the elevation changes. During reinflation, however, the gravity changes are small and opposite in size compared to those expected from the uplift. Hence, a greater volume and/or denser magma is coming into the storage system than would be predicted by the surface deformation measurements.

Gas geochemistry really came into 1 ts own at HVO in 1984. The first good measurements of eruptive gases $\left(\mathrm{H}_{2} \mathrm{O}, \mathrm{H}_{2}, \mathrm{CO}_{2}, \mathrm{CO}, \mathrm{SO}_{2}, \mathrm{H}_{2} \mathrm{~S}, \mathrm{HCl}\right.$, HF $)$ at Mauna Loa, combined with continuous monitoring of gas composition at Kilauea summit and near the eruptive site has yielded the following conclusions, documented by Greenland (1n press) and by Greenland and others (1985).

(1) volatiles arriving at Kilauea summit are enriched in $\mathrm{CO}_{2}$, the least soluble component; from measurements of gas composition and emission flux at fumaroles the content of volatiles in equilibrium with mantle-derived magma may be calculated (Table C-3).

2. Much $\mathrm{CO}_{2}$ is 1 ost during summit storage and therefore magma available for eruption on the rift zone is relatively $\mathrm{SO}_{2}-$ and $\mathrm{H}_{2} \mathrm{O}-\mathrm{rich}$ (Table C-3). 
3. Residual gases left in the lava are dominated by $\mathrm{H}_{2} \mathrm{O}$, the most soluble constituent (Table $\mathrm{C}-3$ ).

4. $\mathrm{H}_{2} \mathrm{O}$ content of analyzed eruptive gases varies widely, partly due to contamination by meteoric water, but also by disequilibrium exsolution accompanying magma transfer (i.e. the exsolution of gases by vesiculation and diffusive transfer is too slow to keep pace with decreasing pressure accompanying rapid rise rates of magma in the eruptive conduit). $\mathrm{CO}_{2}$ and $\mathrm{SO}_{2}$ contents, however, maintain equilibrium at rapidly decreasing pressures and $\mathrm{H}_{2} \mathrm{O}$ contents can be adjusted consistent with this.

5. Gases associated with the Mauna Loa eruption were depleted in $\mathrm{CO}_{2}$, much like the Kilauea rift eruption. This is consistent with the magma chemistry which suggests eruption of the top of a large body of stored magma. It remains to be seen whether a $\mathrm{CO}_{2}$-rich component can be seen during the reinflation of Mauna Loa. Mauna Loa gases differ from Kilauea in their halogen (F;C1) content and ratio, matching subtle differences in the residual halogen contents of Kilauea and Mauna Loa rocks.

The mass of volatiles released by Kilauea magma is measured by COSPEC and can be correlated with magma supply rates. There was a step increase in total volatile mass at the onset of the present Kilauea eruption which agrees with estimates of increased magma supply relative to earlier periods (eg. 1969-1974 Mauna Ulu eruption).

Plans for a new HVO facility became reality in 1984 when Congress passed a bill that included a supplemental appropriation of $\$ 5,000,000$ for the National Park Service construction budget. The plans covered by the budget include 3 stages (1) construction of a new HVO building; (2) renovation of the existing geochemistry wing for HVO use, and (3) renovation of the library-office wing for Park Service use as an on-site interpretation facility. When finished in 1986, this will fulfill much of the original vision of Thomas A. Jaggar, including integration of HVO monitoring and Park Service interpretation.

Changes in staff occurred, including the arrival of a new scientist-in-charge. Bob Decker's going away party was noteworthy as Mauna Loa erupted barely an hour after the party. Tom Wright arrived to take his place about a week before the end of the eruption. Other staff changes are summarized below.

Leavings
\begin{tabular}{lll} 
Name & Position & Going To \\
\hline
\end{tabular}

\begin{tabular}{lc}
\multicolumn{3}{c}{ Arrivings } \\
\hline Name & Position Arrived From \\
\hline
\end{tabular}

\begin{tabular}{|c|c|c|c|c|c|}
\hline $\begin{array}{l}\text { Toni Duggan } \\
\text { Ken }\end{array}$ & Geology & Gradua te School & $\begin{array}{c}\text { Christina } \\
\text { Heliker }\end{array}$ & Geology & CVO \\
\hline Yamashita & Deformation & cVo & George Ulrich & Geology & Flagstaff \\
\hline Ed Wolfe & Geology & $\begin{array}{l}\text { Big Island Map } \\
\text { Project }\end{array}$ & John Hoffmann & Geology & $\begin{array}{l}\text { NAGT }^{1} \\
\text { Program }\end{array}$ \\
\hline Ina Neal & Geology & $\begin{array}{l}\text { Big Island Map } \\
\text { Project }\end{array}$ & & & \\
\hline
\end{tabular}

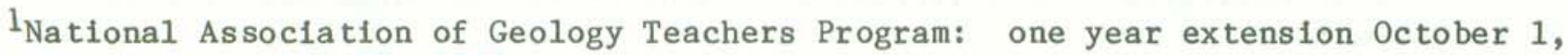
1984 - September 30, 1985. 
Student help in 1984 included the following:

John Hof fmann]

Jim Hutton ] - National Association of Geology Teachers

Teresa Atwill]

Bill Chadwick] - Graduate Students University of California at Santa Barbara

Pauline Tamura]

Joan Yoshioka ] - Federal Junior Fellows

Sandra Zane ]

Brian Moniz ]

Keone Ah Chong

Kaipo Ah Chong

Kent Kikuchi

Charlotte Forbes]

Terry Ignacio ] - Minority Program in the Earth Sciences

Shyanne Nelson]

Tom Tioaquin ] - Alu Like (Native American Program)

Many of the staff took a technical writing course, offerred by Shipley Associates. As a result, we have more persons helping prepare the monthly reports than before. George Kojima and Bob Koyanagi went to Indonesia in August 1984 to evaluate the radio-telemetered seismic network at Merapi Volcano Observatory. This is part of a long term collaboration between the U. S. Geological Survey and the Indonesian government. They were successful in continuing to train Indonesian volcanologists in the use of modern seismic instrumentation and interpretation of Indonesian seismicity.

All in all, 1984 was a productive year for HVO. The challenge of studying eruptions accessible only by helicopter was met with fiscal help in the form of a supplemental appropriation from Congress and, during the Mauna Loa eruption, with observing and monitoring help from HVO alumni and other visiting USGS scientists. 
Table C-1. Summary data, 1984 eruption of Mauna Loa 1

Duration: March 25, 1984 - April 15, 1984

Area covered: $48 \mathrm{~km}^{2}$

Volume ${ }^{2}$ : $.22 \mathrm{~km}^{3}$

Eruption rate (March 26-April 7): $10 \times 10^{6} \mathrm{~m}^{3} /$ day

Channel velocity (April 2-April 7, 8200' elevation): $19 \mathrm{~km} / \mathrm{hr}$

Temperature (maximum reliable readings): $1140 \pm 5^{\circ} \mathrm{C}$

${ }^{1}$ Data from Lockwood et. al. (1985); Lipman and Banks (in press)

${ }^{2}$ Not corrected for vesicularity. 
Table c-2.

SEQ ${ }^{1}$ SEQ 1 EPISODE EPISODE EPISODE EPISODE DAYS EPISODE DEFLAT DEFLAT DEFLAT DEFLAT DEFLAT DEFLAT DEFLAT DEFLAT TILT TOT.TLT UR/HR

$\begin{array}{lllrrrrrrr}\text { EPISODE } & \begin{array}{l}\text { START } \\ \text { DATE }\end{array} & \begin{array}{l}\text { END } \\ \text { DATE }\end{array} & \begin{array}{r}\text { START } \\ \text { M/DA/YR }\end{array} & \begin{array}{r}\text { START } \\ \text { HOUR }\end{array} & \begin{array}{r}\text { END } \\ \text { MMDA } / \text { YR }\end{array} & \begin{array}{c}\text { END } \\ \text { HOUR }\end{array} & \begin{array}{r}\text { REPOSE } \\ \text { PRIOR }\end{array} & \begin{array}{r}\text { LENGTH } \\ \text { HOURS }\end{array} \\ -13 & -383 & 385 & -1 / 20 / 84 & 17: 24 & 1 / 22 / 84 & 11: 23 & 50 & 42 \\ 14 & 393 & 394 & 1 / 30 / 84 & 17: 45 & 1 / 31 / 84 & 13: 18 & 8 & 19 \\ 15 & 408 & 409 & 2 / 14 / 84 & 19: 40 & 2 / 15 / 84 & 15: 01 & 14 & 19 \\ 16 & 426 & 427 & 3 / 03 / 84 & 14: 50 & 3 / 04 / 84 & 22: 31 & 17 & 32 \\ 17 & 453 & 454 & 3 / 30 / 84 & 04: 48 & 3 / 31 / 84 & 03: 24 & 26 & 23 \\ 18 & 472 & 475 & 4 / 18 / 84 & 18: 00 & 4 / 21 / 84 & 05: 33 & 18 & 60 \\ 19 & 500 & 502 & 5 / 16 / 84 & 05: 00 & 5 / 18 / 84 & 00: 50 & 25 & 44 \\ 20 & 522 & 523 & 6 / 07 / 84 & 21: 04 & 6 / 08 / 84 & 06: 25 & 20 & 9 \\ 21 & 545 & 545 & 6 / 30 / 84 & 10: 28 & 6 / 30 / 84 & 18: 27 & 22 & 8 \\ 22 & 553 & 554 & 7 / 08 / 84 & 19: 30 & 7 / 09 / 84 & 10: 17 & 8 & 15 \\ 23 & 573 & 574 & 7 / 28 / 84 & 12: 00 & 7 / 29 / 84 & 05: 40 & 19 & 18 \\ 24 & 595 & 596 & 8 / 19 / 84 & 21: 52 & 8 / 20 / 84 & 17: 25 & 21 & 20 \\ 25 & 626 & 627 & 9 / 19 / 84 & 16: 04 & 9 / 20 / 84 & 05: 32 & 30 & 13.5 \\ 26 & 670 & 670 & 11 / 2 / 84 & 11: 40 & 11 / 2 / 84 & 16: 36 & 42 & 5 \\ 27 & 688 & 688 & 11 / 20 / 84 & 00: 05 & 11 / 20 / 84 & 10: 06 & 18 & 10 \\ 28 & 701 & 702 & 12 / 3 / 84 & 19: 05 & 12 / 4 / 84 & 09: 41 & 13 & 14.5\end{array}$

\begin{tabular}{r} 
LENGTH \\
HOURS \\
\hline 43 \\
24 \\
44 \\
33 \\
51 \\
67.5 \\
51 \\
29 \\
39 \\
28 \\
24 \\
22 \\
19 \\
16 \\
15.5 \\
19
\end{tabular}

\begin{tabular}{lll} 
START & START & S \\
M/DA/YR & HOUR & I \\
\hline $1 / 20 / 84$ & $21: 00$ & 3 \\
$1 / 30 / 84$ & $19: 00$ & 3 \\
$2 / 14 / 84$ & $01: 00$ & 3 \\
$3 / 3 / 84$ & $16: 00$ & 3 \\
$3 / 29 / 84$ & $04: 00$ & 3 \\
$4 / 18 / 84$ & $10: 00$ & 3 \\
$5 / 15 / 84$ & $05: 00$ & 3 \\
$6 / 07 / 84$ & $11: 00$ & 3 \\
$6 / 29 / 84$ & $12: 00$ & 3 \\
$7 / 08 / 84$ & $12: 00$ & 3 \\
$7 / 28 / 84$ & $10: 30$ & 3 \\
$8 / 19 / 84$ & $22: 00$ & 3 \\
$9 / 19 / 84$ & $14: 00$ & 3 \\
$11 / 2 / 84$ & $11: 00$ & 3 \\
$11 / 20 / 84$ & $00: 36$ & 3 \\
$12 / 3 / 84$ & $19: 04$ & 3
\end{tabular}
D.ASM M/DA/YR HOUR ID.ASM HOURS PRIOR MIC.RAD DEFLAT

$31.50 \quad 1 / 22 / 84 \quad 16: 00 \quad 320.40$ $\begin{array}{lll}6.16 & 9.91 & 41\end{array}$ $317.32 \quad 3 / 31 / 84 \quad 07: 00 \quad 306.83 \quad 51-10.47014 .790 .49-21$

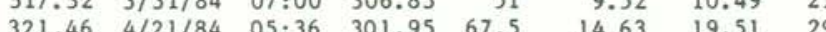
$314.665 / 17 / 84 \quad 08: 00 \quad 308.92 \quad 51 \quad 12.715 .74$ $314.665 / 17 / 84$ 08:00 308.92 $29 \quad 12.710 .740$ $315.36 \quad 7 / 01 / 84 \quad 03: 00 \quad 306.01 \quad 3907.56 \quad 9.35$

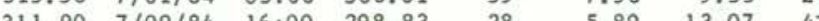

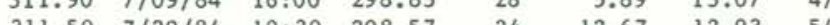
$309.08 \quad 7 / 29 / 84$ 10:30 298.57 $24 \quad 12.67 \quad 12.93 \quad 54$

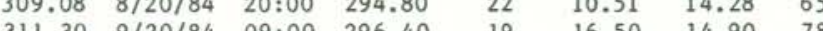

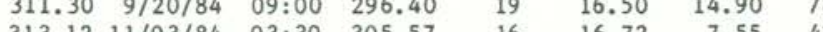

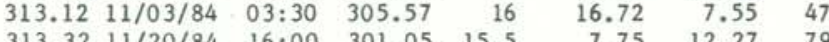

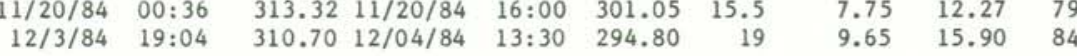

\begin{tabular}{|c|c|c|c|c|c|c|c|c|c|c|c|c|}
\hline EPISODE & $\begin{array}{l}\text { AREA } \\
\text { SQ KM }\end{array}$ & $\begin{array}{l}\text { VOLUME } \\
\text { MILL. } \\
\text { CU.MTRS }\end{array}$ & $\begin{array}{l}\text { RATE } \\
1000 \mathrm{CU} \\
\text { MTRS/HR }\end{array}$ & $\begin{array}{c}\text { AVG } \\
\text { THICK } \\
\text { MTRS } \\
\end{array}$ & $\begin{array}{c}\mathrm{SO}_{2} \\
\text { EMISS. } \\
\text { TONS/HR }\end{array}$ & $\begin{array}{l}\text { RATE } \\
1000 \text { CU MTRS } \\
\text { PER MICRORAD }\end{array}$ & $\begin{array}{l}\text { TREMOR } \\
\text { START } \\
\text { M/DA/YR }\end{array}$ & $\begin{array}{r}\text { TREM } \\
\text { START } \\
\text { HOUR }\end{array}$ & $\begin{array}{c}\text { TREM } \\
\text { END } \\
\text { M/DA/YR }\end{array}$ & $\begin{array}{r}\text { TREM } \\
\text { END } \\
\text { HOUR }\end{array}$ & $\begin{array}{l}\text { TR.ST. } \\
\text { SEQ. } \\
\text { DATE }\end{array}$ & $\begin{array}{l}\text { TR. END } \\
\text { SEQ } \\
\text { DATE }\end{array}$ \\
\hline 13 & 2.6 & 10 & 238 & 3.00 & ND & 901 & $1 / 20 / 84$ & $14: 00$ & $1 / 22 / 84$ & $11: 19$ & 383 & 385 \\
\hline 14 & 2.1 & 6 & 316 & 2.95 & $>490$ & 605 & $1 / 30 / 84$ & $18: 10$ & $1 / 31 / 84$ & $13: 19$ & 393 & 394 \\
\hline 15 & 2.2 & 8 & 421 & 3.64 & 1100 & 690 & $2 / 14 / 84$ & $19: 46$ & $2 / 15 / 84$ & $15: 01$ & 408 & 409 \\
\hline 16 & 3.2 & 12 & 375 & 3.72 & 750 & 812 & $3 / 03 / 84$ & $14: 35$ & $3 / 04 / 84$ & $22: 29$ & 426 & 427 \\
\hline 17 & 3.0 & 10 & 435 & 3.20 & $>380$ & 953 & $3 / 30 / 84$ & $05: 10$ & $3 / 31 / 84$ & $03: 16$ & 453 & 454 \\
\hline 18 & 6.6 & 24 & 400 & 3.88 & ND & 1230 & $4 / 18 / 84$ & $14: 00$ & $4 / 21 / 84$ & $05: 35$ & 472 & 475 \\
\hline 19 & 1.4 & 2 & 45 & 1.63 & ND & 348 & $5 / 16 / 84$ & $08: 16$ & $5 / 17 / 84$ & $18: 00$ & 500 & 501 \\
\hline 20 & 1.6 & 4 & 444 & 2.56 & ND & 388 & $6 / 07 / 84$ & $21: 20$ & $6 / 08 / 84$ & $06: 27$ & 522 & 523 \\
\hline 21 & 1.92 & 5.7 & 713 & 2.97 & 1200 & 610 & $6 / 30 / 84$ & $10: 28$ & $6 / 30 / 84$ & $18: 27$ & 545 & 545 \\
\hline 22 & 2.69 & 7.7 & 513 & 2.86 & ND & 589 & $7 / 08 / 84$ & $18: 59$ & $7 / 09 / 84$ & $10: 17$ & 553 & 554 \\
\hline 23 & 3.28 & 9.5 & 528 & 2.90 & ND & 735 & $7 / 28 / 84$ & $11: 58$ & $7 / 29 / 84$ & $05: 43$ & 573 & 574 \\
\hline 24 & 3.73 & 11.6 & 580 & 3.11 & ND & 812 & $8 / 19 / 84$ & $21: 25$ & $8 / 20 / 84$ & $17: 21$ & 595 & 596 \\
\hline 25 & 3.33 & 11.1 & 822 & 3.33 & ND & 745 & $9 / 19 / 84$ & $15: 30$ & $9 / 20 / 84$ & $05: 32$ & 625 & 626 \\
\hline 26 & 1.64 & 6.6 & 1320 & 4.02 & ND & 874 & $11 / 02 / 8$ & $11: 40$ & $11 / 02 / 84$ & $16: 34$ & 670 & 670 \\
\hline 27 & 2.30 & 8.4 & 840 & 3.65 & ND & 685 & $11 / 20 / 8$ & $00: 05$ & $11 / 20 / 84$ & $10: 08$ & 688 & 688 \\
\hline 28 & 3.62 & 12.4 & 855 & 3.43 & 1300 & 780 & $12 / 03 / 8$ & $19: 05$ & $12 / 04 / 84$ & $09: 41$ & 701 & 702 \\
\hline
\end{tabular}

$1_{\text {Days since January } 2,1983}$ 
Table C-3. $\mathrm{H}_{2} \mathrm{O}-\mathrm{CO}_{2}-\mathrm{S}$ budget in Kilauea magma

$\begin{array}{ccc}\mathrm{H}_{2} \mathrm{O} \text { (wt. \%) } & \mathrm{CO}_{2} \text { (wt. \%) } & \text { S (wt. \%) } \\ .32 & .32 & .09 \\ .28 & .03 & .08 \\ .05 & & .02\end{array}$

Magma arriving from mantle

Magma after storage and before eruption

Residual volatiles in erupted magma
.05

$<.01$ 


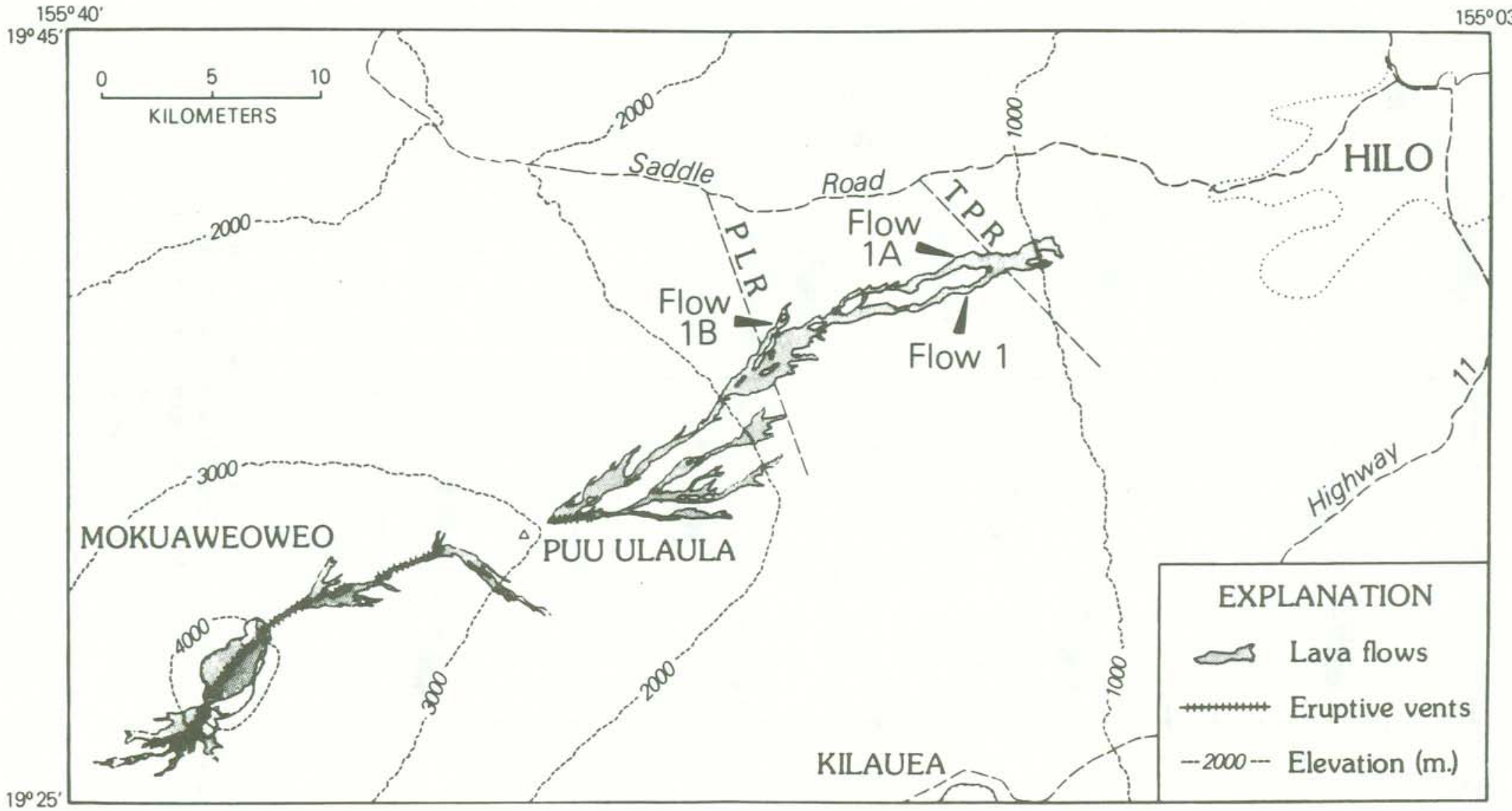

Figure C-1. Distribution of lava flows, 1984 eruption of Mauna Loa volcano (from Lockwood and others, 1985, Fig. 5). 

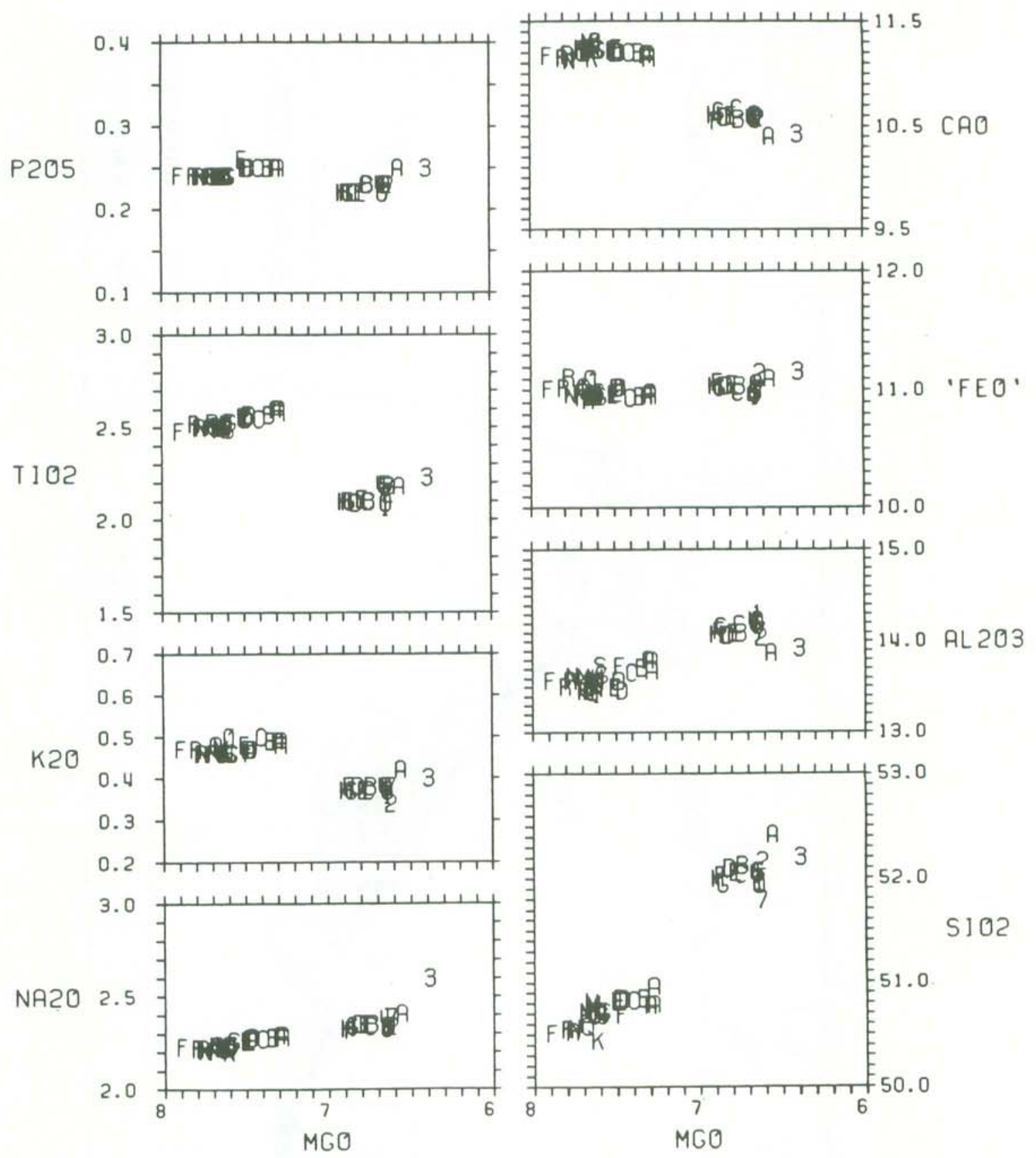

Figure C-2. MgO variation diagrams showing contrasting chemistry of Kilauea (left-hand group) and Mauna Loa (right-hand group) during 1984. Mauna Loa group shows similarity of1984 (letters A-H) and 1975 (numerals 0-7) compositions. The spread of Kilauea data is due principally to differences in olivine content of different eruptive episodes. 


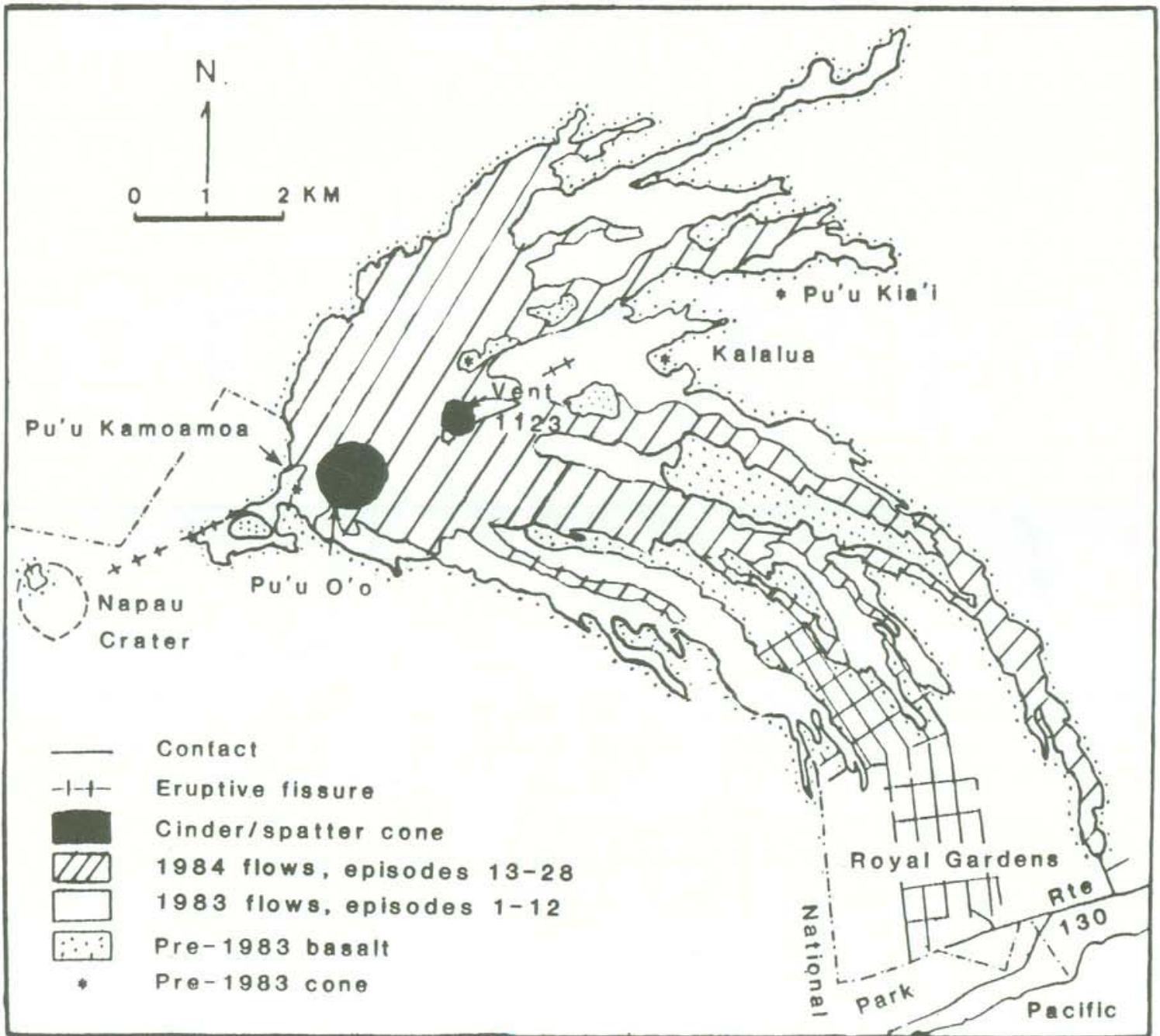

Figure C-3. Distribution of lava flows erupted from Pu'u O'o in 1984. 


\section{SUMMARY - KILAUEA}

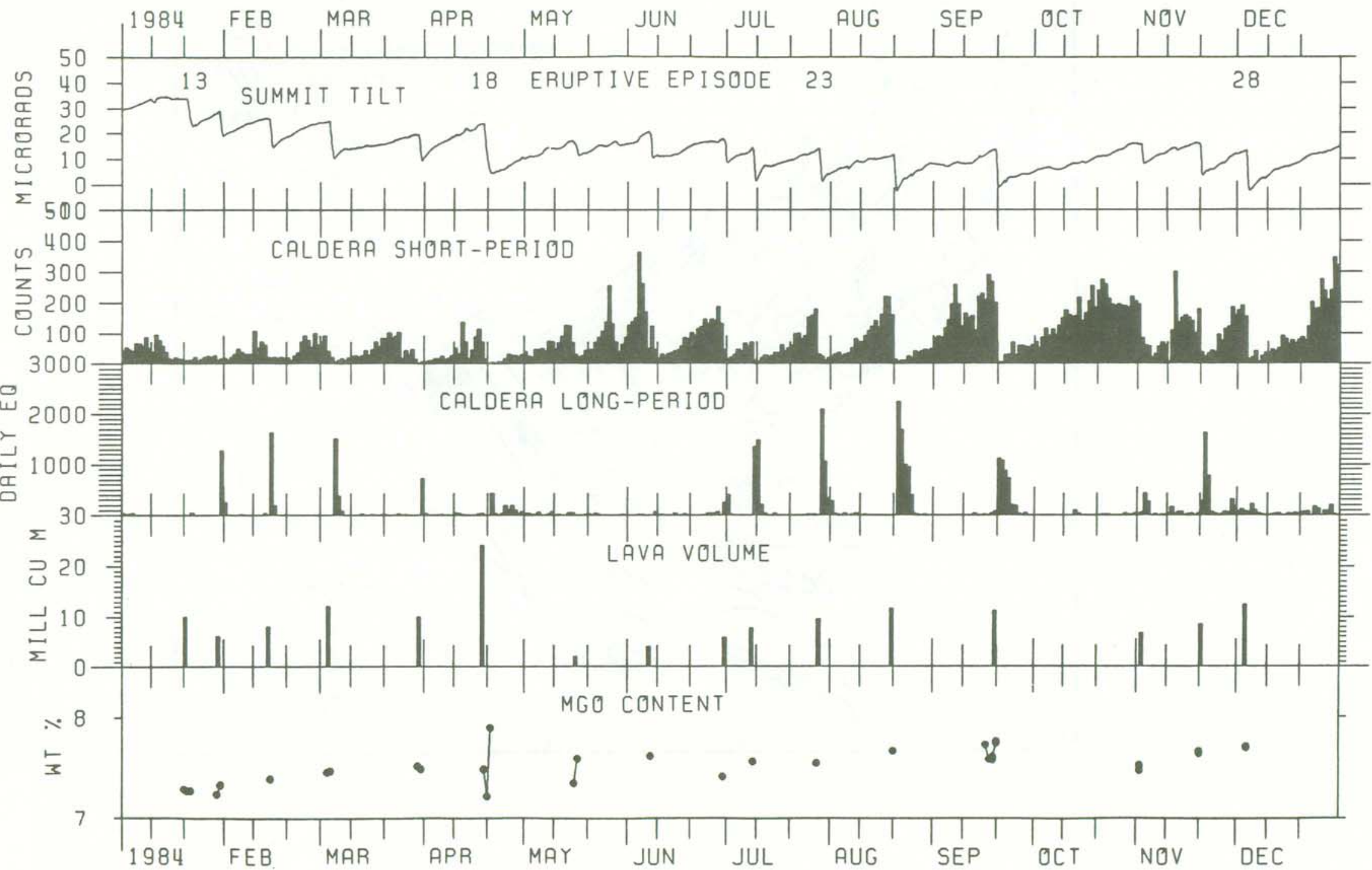

Figure C-4. Summary of selected geologic, geodetic, and seismic data associated with Kilauea eruptive episodes in 1984. 
Decker, R.W., Koyanagi, R.Y., Dvorak, J.J., Lockwood, J.P., Okamura, A.T., Yamashita, K.M., and Tanigawa, W.R., 1983, Seismicity and surface deformation of Mauna Loa volcano, Hawa11: EOS, v. 64, p. 545-547.

Greenland, L.P., 1986, Composition of eruptive gases from Mauna Loa volcano, Hawa11: U.S. Geological Survey Professional Paper 1350.

Greenland, L.P., Rose, W.I., and Stokes, J.B., 1985, An estimate of gas emissions and magmatic gas content from Kilauea volcano: Geochimi. et Cosmochim. Acta, v. 49, p. 125-129.

Johnson, D.J., 1986, Elastic and inelastic magma storage in Kilauea volcano, Hawail: U.S. Geological Survey Professional Paper 1350.

Lipman, P.W., and Banks, N.G., 1986, A'a flow dynamics, Mauna Loa, 1984: U.S. Geological Survey Professional Paper 1350.

Lockwood, J.P., Banks, N.G., English, T.T., Greenland, L.P., Jackson, D.B., Johnson, D.J., Koyanag1, R.Y., Okamura, A.T., and Rhodes, J.M., 1985, The 1984 eruption of Mauna Loa volcano, Hawa11: EOS, v. 66, p. 169171 .

Ryan, M., 1986, The elasticity and expansivity of Hawalian olivine tholeifte and 1 ts role in the stability and structural evolution of intrashield magma reservoirs and rift systems: (I) Emperiments to $1223 \mathrm{~K}$; (II) Mixing models for computation; and (III) The pressure dependence of contractancy: U.S. Geological Survey Professional Paper 1350. 


\section{SEISMIC INSTRUMENTATION}

The network. The Hawailan Volcano Observatory maintains an extensive telemetering seismometer network on the island of Hawail. In 1984 the seismometer network consisted of 50 stations; two are low-gain multicomponent stations (optical), twelve are three-component, and 36 are vertical only. The coverage is most complete on and around Kilauea Volcano. With the exception of self-contained systems at Uwekahuna and Hilo stations, all seismometer signals from the short period network are telemetered to the observatory for recording.

Figure 1 is a map of selected geographic and geologic features, Figure 2 shows the seismic stations which were operated on the Island of Hawaif during the year, and Figure 3 indicates the telemetry scheme for the respective seismic stations. Table 1 lists all sefsmic stations operated by the U.S. Geological Survey in Hawaii during 1984. Listed are names, three- and four-letter codes, coordinates in degrees and minutes, elevation in meters, and other data as described below pertaining to each station. In addition to seismometers listed in Table 1, a horizontal seismometer of Type 3 and a long-period, three-component set of Press-Ewing seismometers are operated in the Uwekahuna vault, all recorded on photographic paper.

Instrumentation and recording. Each telemetered station has a voltage controlled oscillator (VCO) for FM multiplex transmission to HVO via either hardwire or radio. These telemetering stations are now all of Type 1 , the OEVE standard system used in USGS seismic networks (see Table 2 for details). After discrimination at the receiver, the analog signals from 36 stations are recorded on two Develocorders using $16 \mathrm{~mm}$ microfilm. FM signals from the telemetering network are also recorded directly on one-inch magnetic tape. Selected larger events are copied on to condensed FM library tapes which are currently archived in Menlo Park and archived in digital form at HVO as part of the routine location processing. The type of recording used for each station (in addition to magnetic tape for the telemetered stations) is coded in Table 1 as follows: D Develocorder film, S - smoked paper drum, P - photographic paper, H - Helicorder paper.

In addition to the standard stations, optical drum seismographs are maintained at Uwekahuna (HVO), Hilo, Maui, and on Oahu (Honolulu station operated by the Pacific Tsunami Warning Center). The less sensitive optical records are used primarily for amplitude measurements for magnitude calculations to supplement readings from the high-gain stations. The paper records as well as the $16 \mathrm{~mm}$ Develocorder microfilm are archived at HVO.

In la te August, 1982, Rex Allen and Jim Ellis from the U.S. Geological Survey's Office of Earthquake Studies came to HVO to install their seismic monitoring system uniquely designed to automate picking of P-phase onset times, measuring coda lengths, and determinining preliminary epicenters for adequately recorded earthquakes. The Hawail picker is configured to monitor a maximum of 80 stations, but is currently wired to $45 \mathrm{stations.} \mathrm{Attached} \mathrm{to} \mathrm{the} \mathrm{picker} \mathrm{are}$ a Datasouth Printer and an Apple II microcomputer. The picker output is logged on a disk and printed out on chart paper within three minutes after the earthquake occurs. The data collected on disk is tranferred to the Eclipse, the main computer system, on a daily basis using a second Apple II. The earthquake records are filtered and run through our standard location program (HYPOINVERSE). Summary listings and epicenter plots are prepared on chart 
paper, and the phase data are archived on tape. The picker output is routinely compared with readings from our standard format.

Seismograph response and calibration. Displacement response curves for the four short-period seismograph types in use are given in Figure 4. Types three and four are electro-mechanical systems recorded on paper records. The Type 1 curve gives the displacement magnification of the standard OEVE system from ground motion at the seismometer to the seismic trace as seen on a $20 \mathrm{x}$ Develocorder film viewer. The curves plot the unit response which should be multiplied by a constant but known factor (CAL, Table 1) to get the response for an individual station. Individual CAL factors for Type 1 seismographs are equal to the peak-to-peak amplitude measured in $\mathrm{mm}$ on the $20 \mathrm{X}$ Develocorder viewer of a 10 microvolt $5 \mathrm{~Hz}$ signal introduced to the preamp/VCO in place of the geophone. Calibration is normally done each time a station is visited. CAL factors range from about 1 to 8, averaging about 4. A detalled history of CAL factors and other data is given in F.W. Klein and R.Y. Koyanagi, Hawailan Volcano Observatory Seismic Network History 1950-79, U.S. Geological Survey Open File Report 80-302, 1980. 


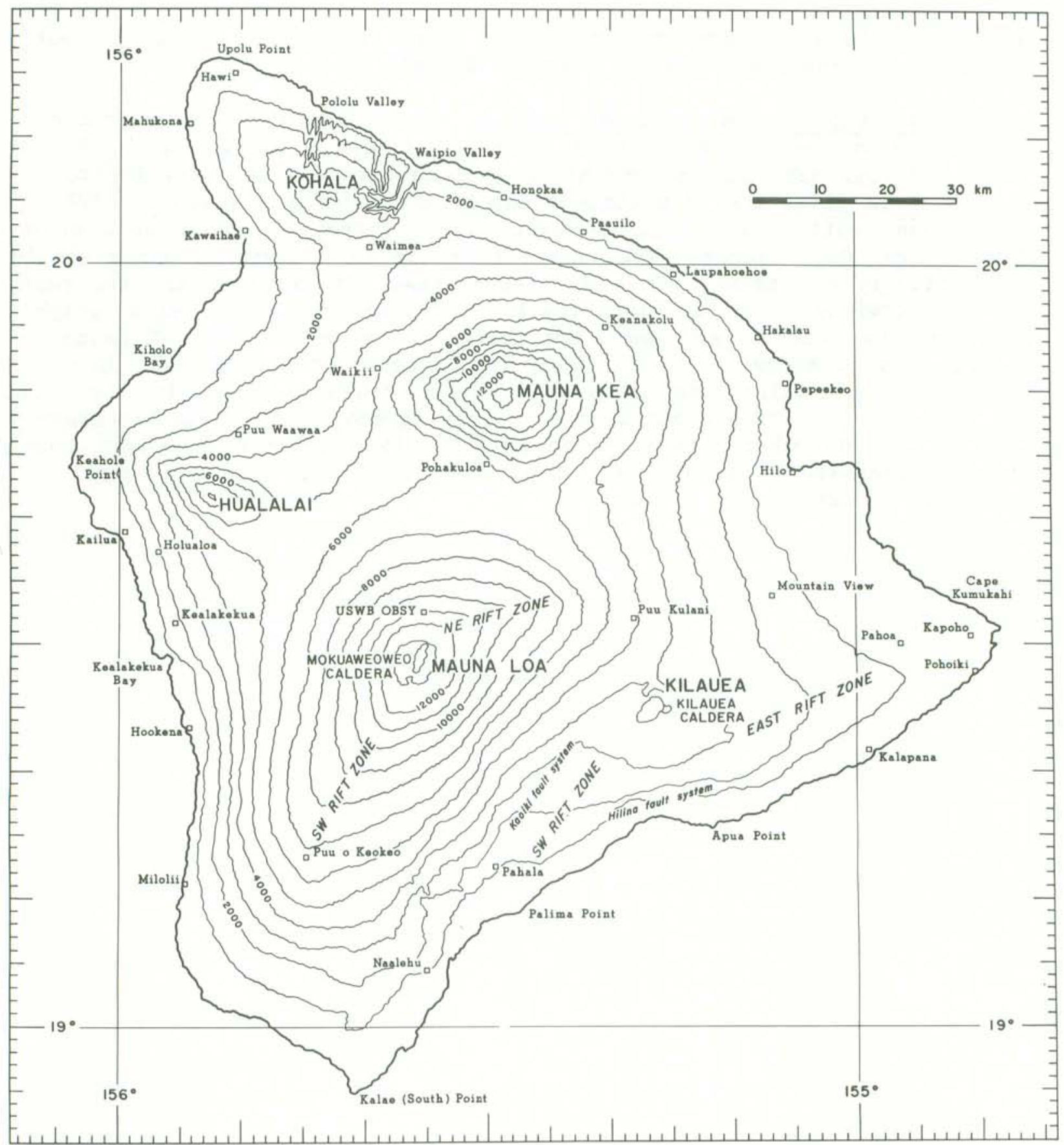

Figure 1. Map of the island of Hawail showing principal settlements and selected geographic and geologic features. 


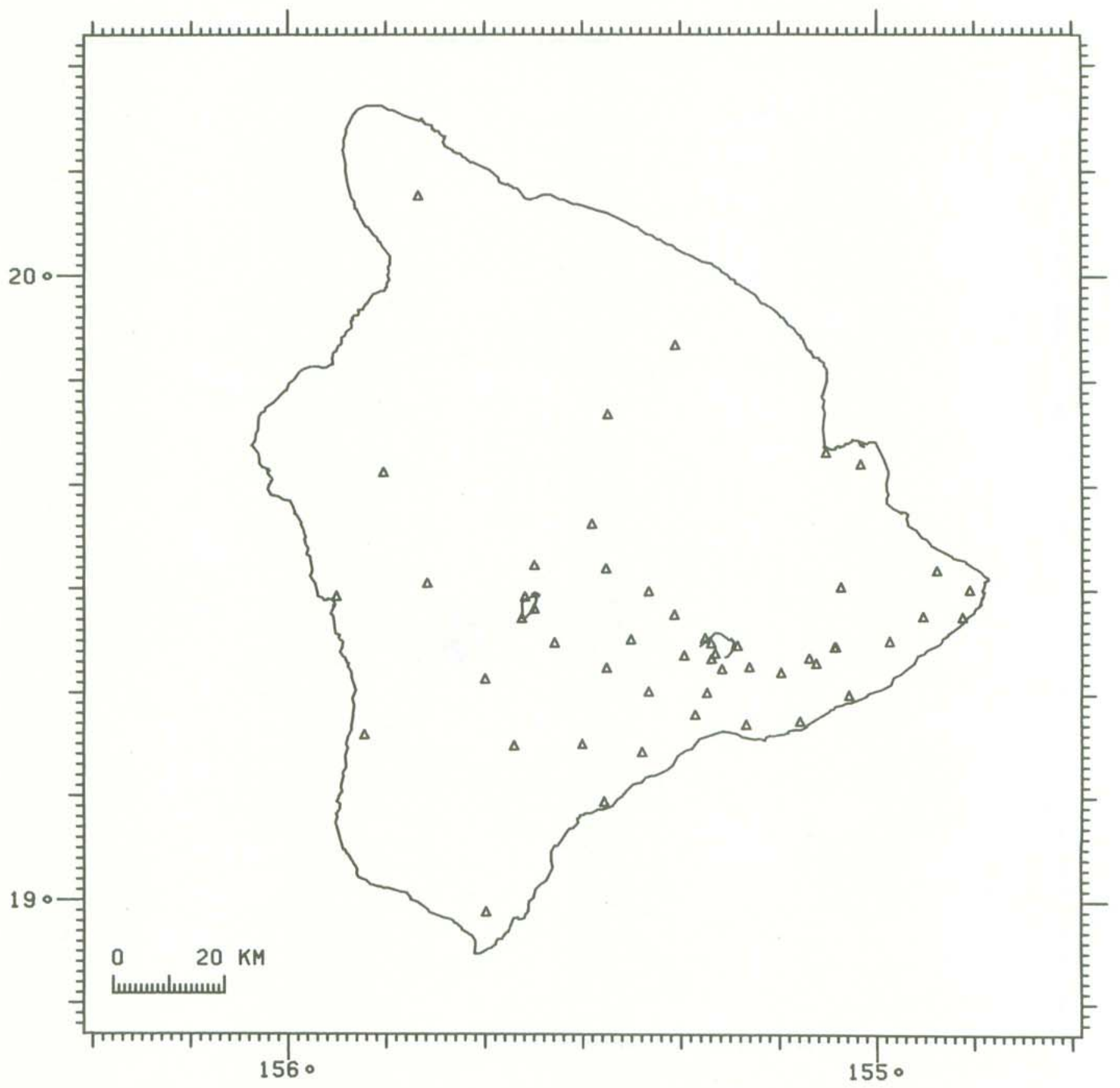

Figure 2. Map of the island of Hawail showing seismic stations operational during 1984. 


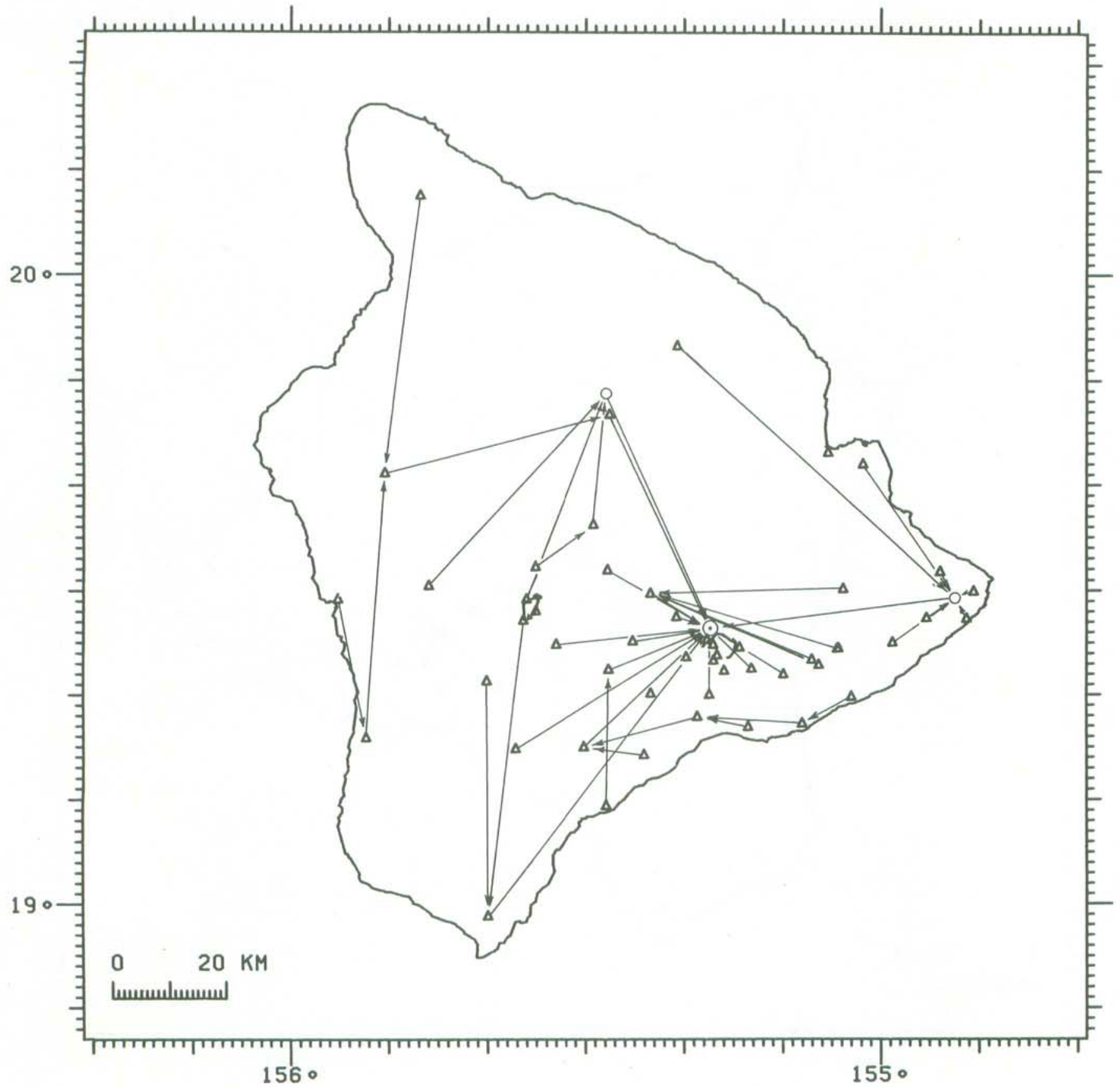

Figure 3. Map of the 1sland of Hawa11 showing the telemetry scheme for the Hawailan Volcano Observatory seismic network.

Legend $\quad \Delta$ Seismometer location

- Repeater Station 
Table 1. Seismometer stations in Hawail operated by the U.S. Geological Survey, 1984.

\begin{tabular}{|c|c|c|c|c|c|c|c|c|c|c|c|c|}
\hline \multirow[t]{2}{*}{ STATION NAME } & \multirow{2}{*}{\multicolumn{2}{|c|}{ CODE }} & \multicolumn{2}{|c|}{ - LAT . . } & \multicolumn{2}{|c|}{$\cdots$ LON $\cdots$} & \multirow{2}{*}{$\begin{array}{r}\text { ELEV } \\
\text { (M) }\end{array}$} & \multirow{2}{*}{$\begin{array}{c}\text { DELAY } \\
1\end{array}$} & \multirow{2}{*}{$\begin{array}{c}\text { DELAY } \\
2\end{array}$} & \multirow[t]{2}{*}{ CAL } & \multirow{2}{*}{$\begin{array}{l}\text { SEIS } \\
\text { TYPE }\end{array}$} & \multirow{2}{*}{$\begin{array}{l}\text { OPTIC } \\
\text { RECORD }\end{array}$} \\
\hline & & & D & M & D & M & & & & & & \\
\hline 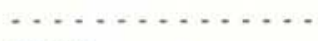 & & - & $\ldots$ & $\ldots$ & 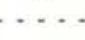 & 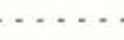 & & & & & & \\
\hline AHUA & $\mathrm{AHU}$ & $\mathrm{z}$ & 19 & 22.40 & 155 & 15.90 & 1070 & -0.10 & -0.13 & 2.1 & E4 & SD \\
\hline AHUA & AHUE & $E$ & 19 & 22.40 & 155 & 15.90 & 1070 & -0.10 & -0.13 & 1.0 & E4 & \\
\hline AHUA & AHUN & $\mathrm{N}$ & 19 & 22.40 & 155 & 15.90 & 1070 & .0 .10 & .0 .13 & 1.0 & E4 & \\
\hline A INAPO & AIN & $\mathrm{z}$ & 19 & 22.50 & 155 & 27.62 & 1524 & 0.13 & 0.17 & 5.5 & L 4 & D \\
\hline AINAPO & AINE & $E$ & 19 & 22.50 & 155 & 27.62 & 1524 & 0.13 & 0.17 & 1.0 & L4 & \\
\hline AINAPO & AINN & $\mathrm{N}$ & 19 & 22.50 & 155 & 27.62 & 1524 & 0.13 & 0.17 & 1.0 & L4 & \\
\hline CAPTAIN COOK & CAC & $\mathrm{Z}$ & 19 & 29.29 & 155 & 55.09 & 323 & 0.00 & -0.16 & 1.1 & L 4 & D \\
\hline CONE PEAK & CPK & $\mathrm{Z}$ & 19 & 23.70 & 155 & 19.70 & 1038 & -0.26 & .0 .07 & 6.0 & L4 & \\
\hline DANDELION & DAN & $\mathrm{z}$ & 19 & 21.42 & 155 & 40.04 & 3003 & -0.27 & 0.03 & 7.0 & L4 & $D$ \\
\hline DESERT & DES & $\mathrm{z}$ & 19 & 20.20 & 155 & 23.30 & 815 & -0.29 & -0.13 & 3.0 & E4 & $S D$ \\
\hline ESCAPE ROAD & ESR & $\mathrm{Z}$ & 19 & 24.68 & 155 & 14.33 & 1177 & -0.17 & -0.19 & 2.2 & L4 & D \\
\hline HAWAI IAN BEACHE & ES HAB & $\mathrm{Z}$ & 19 & 31.89 & 154 & 53.89 & 92 & -0.09 & -0.24 & 1.0 & L4 & \\
\hline HALEAKALA, MAUI & I HAE & $\mathrm{E}$ & 20 & 46.00 & 156 & 15.00 & 2090 & 0.00 & 0.00 & 1.0 & W & $\mathrm{P}$ \\
\hline HALEAKALA， MAUI & HAL & $\mathrm{Z}$ & 20 & 46.00 & 156 & 15.00 & 2090 & 0.00 & 0.00 & 0.7 & $\mathrm{HI}$ & $\mathrm{P}$ \\
\hline HALEAKALA， MAUI & HAN & $\mathrm{N}$ & 20 & 46.00 & 156 & 15.00 & 2090 & 0.00 & 0.00 & 1.0 & w & P \\
\hline HILO & HIE & $\mathrm{E}$ & 19 & 43.20 & 155 & 5.30 & 20 & 0.54 & 0.30 & 1.0 & W & $\mathrm{P}$ \\
\hline HILO & HIL & $\mathrm{z}$ & 19 & 43.20 & 155 & 5.30 & 20 & 0.54 & 0.30 & 1.0 & $\mathrm{HI}$ & $\mathrm{P}$ \\
\hline HILO & HIN & $\mathrm{N}$ & 19 & 43.20 & 155 & 5.30 & 20 & 0.54 & 0.30 & 1.0 & w & $\mathrm{P}$ \\
\hline HILINA PALI & HLP & $\mathrm{Z}$ & 19 & 17.96 & 155 & 18.63 & 707 & 0.02 & 0.07 & 2.6 & L4 & D \\
\hline HONOLULU, OAHU & HON & $\mathrm{z}$ & 21 & 19.30 & 158 & 0.50 & 2 & 0.00 & 0.00 & 0.0 & $\mathrm{HI}$ & $\mathrm{P}$ \\
\hline HALE POHAKU & HPU & $\mathrm{Z}$ & 19 & 46.85 & 155 & 27.50 & 3396 & 0.31 & 0.17 & 3.3 & L4 & D \\
\hline HUMUULA SHEEP S & ST HSS & $\mathrm{Z}$ & 19 & 36.31 & 155 & 29.13 & 2445 & 0.20 & 0.35 & 5.3 & L4 & D \\
\hline HUMUULA SHEEP S & STHSSE & $\mathrm{E}$ & 19 & 36.31 & 155 & 29.13 & 2445 & 0.20 & 0.35 & 1.0 & L & \\
\hline HUMUULA SHEEP S & STHSSN & $\mathrm{N}$ & 19 & 36.31 & 155 & 29.13 & 2445 & 0.20 & 0.35 & 1.0 & L & \\
\hline HOT CAVES & HTC & $\mathrm{Z}$ & 19 & 14.33 & 155 & 24.02 & 381 & -0.16 & -0.07 & 0.0 & E4 & \\
\hline HUALALAI & HUA & $\mathrm{z}$ & 19 & 41.25 & 155 & 50.32 & 2189 & 0.67 & 0.38 & 3.0 & L 4 & D \\
\hline HEIHEIAHULU & HUL & $\mathrm{z}$ & 19 & 25.13 & 154 & 58.72 & 369 & -0.17 & -0.16 & 1.6 & E4 & DS \\
\hline HEIHEI AHULU & HULE & E & 19 & 25.13 & 154 & 58.72 & 369 & -0.17 & -0.16 & 1.0 & E 4 & \\
\hline HEIHEIAHULU & HULN & $\mathrm{N}$ & 19 & 25.13 & 154 & 58.72 & 369 & -0.17 & -0.16 & 1.0 & L4 & \\
\hline KAAPUNA & KAA & $\mathrm{z}$ & 19 & 15.98 & 155 & 52.28 & 524 & -0.12 & -0.01 & 3.5 & L4 & D \\
\hline KAENA POINT & KAE & $\mathrm{z}$ & 19 & 17.35 & 155 & 7.95 & 37 & -0.01 & 0.06 & 1.4 & L 4 & D \\
\hline KAHAUALEA & $\mathrm{KAH}$ & $\mathrm{z}$ & 19 & 24.58 & 155 & 4.36 & 625 & -0.25 & -0.30 & 0.0 & L 4 & $\mathrm{DH}$ \\
\hline KAOIKI FAULTS & KFA & 2 & 19 & 25.26 & 155 & 25.14 & 1579 & 0.13 & 0.17 & 0.0 & $E$ & \\
\hline KAHUKU & $\mathrm{KHU}$ & 2 & 19 & 14.90 & 155 & 37.10 & 1939 & 0.03 & .0 .03 & 2.7 & E4 & D \\
\hline KANEKI I & K I I & $\mathrm{z}$ & 19 & 30.56 & 155 & 45.90 & 1841 & 0.15 & 0.37 & 2.9 & L 4 & D \\
\hline KANEK I I & KIIE & $E$ & 19 & 30.56 & 155 & 45.90 & 1841 & 0.15 & 0.37 & 1.0 & L & \\
\hline KANEK I I & KIIN & $\mathrm{N}$ & 19 & 30.56 & 155 & 45.90 & 1841 & 0.15 & 0.37 & 1.0 & L & \\
\hline KEANAKOLU & KKU & $\mathrm{z}$ & 19 & 53.39 & 155 & 20.58 & 1863 & 0.68 & 0.24 & 3.3 & L 4 & D \\
\hline PUU KALIU & KLU & $\mathrm{z}$ & 19 & 27.48 & 154 & 55.26 & 271 & -0.17 & -0.30 & 2.9 & L4 & D \\
\hline KAMOAMOA & KMM & $\mathrm{z}$ & 19 & 23.47 & 155 & 6.98 & 750 & -0.25 & -0.30 & 2.4 & L 4 & $\mathrm{DH}$ \\
\hline KAMOAMOA EAST-W & NEKMME & $\mathrm{E}$ & 19 & 23.47 & 155 & 6.98 & 750 & -0.25 & -0.30 & 1.0 & L 4 & \\
\hline KAMOAMOA NORTH - & - SKMMN & $\mathrm{N}$ & 19 & 23.47 & 155 & 6.98 & 750 & -0.25 & -0.30 & 1.0 & L 4 & \\
\hline KOHALA & $\mathrm{KOH}$ & $\mathrm{z}$ & 20 & 7.69 & 155 & 46.77 & 1166 & -0.03 & -0.17 & 1.5 & L 4 & D \\
\hline KOHALA & КОНЕ & $\mathrm{E}$ & 20 & 7.69 & 155 & 46.77 & 1166 & -0.03 & -0.17 & 2.2 & L 4 & \\
\hline KOHALA & KOHN & $\mathrm{N}$ & 20 & 7.69 & 155 & 46.77 & 1166 & -0.03 & -0.17 & 2.2 & L 4 & \\
\hline KI PUKA NENE & KPN & $\mathrm{z}$ & 19 & 20.10 & 155 & 17.40 & 924 & -0.11 & -0.08 & 3.5 & E4 & D \\
\hline KAРОHO & KPO & $\mathrm{z}$ & 19 & 30.02 & 154 & 50.51 & 134 & -0.09 & -0.24 & 2.5 & L4 & D \\
\hline MAUNA LOA & MLO & $\mathrm{z}$ & 19 & 29.80 & 155 & 23.30 & 2010 & 0.03 & 0.08 & 5.8 & L4 & SD \\
\hline MAUNA LOA & MLOE & $\mathrm{E}$ & 19 & 29.80 & 155 & 23.30 & 2010 & 0.03 & 0.08 & 0.0 & L 4 & \\
\hline MAUNA LOA & MLON & $\mathrm{N}$ & 19 & 29.80 & 155 & 23.30 & 2010 & 0.03 & 0.08 & 1.5 & L 4 & \\
\hline MAUNA LOA $X$ & MLX & $\mathrm{z}$ & 19 & 27.60 & 155 & 20.70 & 1475 & 0.06 & 0.15 & 3.0 & L 4 & \\
\hline MOKUAWEOWEO & MOK & $\mathrm{z}$ & 19 & 29.28 & 155 & 35.98 & 4104 & 0.15 & 0.16 & 5.5 & L 4 & D \\
\hline MAKAOPUHI & MPR & $\mathrm{z}$ & 19 & 22.07 & 155 & 9.85 & 881 & -0.17 & -0.20 & 4.2 & L 4 & D \\
\hline MOUNTAIN VIEW & MTV & $\mathrm{z}$ & 19 & 30.25 & 155 & 3.75 & 409 & -0.02 & 0.01 & 5.0 & E4 & D \\
\hline NATIONAL GUARD & NAG & $\mathrm{z}$ & 19 & 42.12 & 155 & 1.72 & 18 & 0.54 & 0.30 & 3.2 & E4 & D \\
\hline NORTH PIT & NPT & $\mathrm{z}$ & 19 & 24.90 & 155 & 17.00 & 1115 & -0.30 & -0.18 & 3.0 & E4 & SD \\
\hline NORTH PIT & NPTE & $\mathrm{E}$ & 19 & 24.90 & 155 & 17.00 & 1115 & -0.30 & -0.18 & 0.0 & E4 & \\
\hline NORTH PIT & NPTN & $\mathrm{N}$ & 19 & 24.90 & 155 & 17.00 & 1115 & -0.30 & .0 .18 & 0.0 & E4 & \\
\hline OUTLET & OTL & $\mathrm{z}$ & 19 & 23.38 & 155 & 16.94 & 1038 & -0.19 & -0.18 & 4.9 & L 4 & \\
\hline
\end{tabular}


Table 1 (continued)

\begin{tabular}{|c|c|c|c|c|c|c|c|c|c|c|c|}
\hline AUAHI & PAU & $\mathrm{z}$ & 19 & 22.62 & 155 & 13.10 & 994 & -0.21 & .0 .24 & 2.4 & L4 4 \\
\hline PAUAHI & PAUE & $\mathrm{E}$ & 19 & 22.62 & 1 & 13.10 & 994 & -0.21 & -0.24 & 0.0 & L4 \\
\hline PAUAH I & PAUN & $\mathrm{N}$ & 19 & 22.62 & 155 & 13.10 & 994 & -0.21 & -0.24 & 0.0 & L4 \\
\hline PUU ULAULA & PLA & $\mathrm{z}$ & 19 & 32.00 & & 27.67 & 2992 & -0.03 & 0.13 & 5.4 & L \\
\hline POHOIKI & POI & $\mathrm{z}$ & 10 & 27.42 & & 51.22 & 16 & -0.09 & -0.24 & 0. & L 4 \\
\hline POLIOKEAWE PALI & POL & $\mathrm{z}$ & 9 & 17.02 & 5 & 13.47 & 169 & -0. & 0.03 & 2.8 & 4 \\
\hline PUU PILI & PPL & 7 & 9 & 9.50 & & .87 & 5 & -0 & -0 & 7 & 4 \\
\hline RIM & RIM & $\mathrm{Z}$ & 9 & 23.90 & & .60 & 1128 & -0.21 & .0 .13 & 0.0 & 4 \\
\hline SOUTH POINT & SPT & $\mathrm{z}$ & 8 & 58.91 & & 39.92 & 244 & -0.17 & -0.22 & 2.8 & 4 \\
\hline SOUTH POINT & SPTE & $E$ & 8 & 1 & & & & -0. & -0.22 & U. & 4 \\
\hline SOUTH POINT & SPTN & $\mathrm{N}$ & 0 & & & & & -0.17 & -0.22 & & L 4 \\
\hline SOUTHWEST RIFT & SWR & $\mathrm{z}$ & 9 & & & & 048 & & & & E4 \\
\hline TRAIL & TRA & $\mathrm{Z}$ & & 1 & & & 7 & & 0 & 0 & L4 \\
\hline UWEKAHUNA & UEE & $E$ & & $2 !$ & & 1 & 10 & -0 & 0.00 & 2.0 & $\varepsilon$ \\
\hline UWEKAHUNA & UEN & $\mathrm{N}$ & & 25 & & 0 & 1240 & -0.21 & 0.00 & 2.0 & $E$ \\
\hline UWEKAHUNA & UEZ & $\mathrm{z}$ & & 25 & & 0 & 1240 & -0.21 & 0.00 & 2.0 & $E$ \\
\hline WAIKI I & WAI & $\mathrm{Z}$ & & 51.58 & & 39.60 & 1433 & 0.20 & 0.35 & 0.0 & L 4 \\
\hline WAHAULA & WHA & $\mathrm{z}$ & 19 & 19.90 & & 2.92 & 29 & -0.10 & -0.04 & 1.5 & E4 \\
\hline WILKES CAMP & WIL & $\mathrm{z}$ & 19 & 28.15 & & 35.02 & 4037 & 0.22 & 0.17 & 2.6 & E4 \\
\hline WILKES CAMP EASTh & WILE & $E$ & & & & & 4037 & & 0.17 & 0.0 & L \\
\hline WILKES CAMP NORTh & WI LN & $\mathrm{N}$ & & & & & & .22 & 0.17 & 0.0 & L \\
\hline WEATHER OBSERVAT & WOB & $\mathrm{z}$ & & & & & 3396 & & 0.00 & 0.0 & E4 \\
\hline WOOD VALLEY & woo & $z$ & 19 & 15.08 & & 30.12 & 909 & .0 .15 & -0.06 & 4.6 & E4 \\
\hline
\end{tabular}

Table 2. Seismic Instrumentation Types

The codes in parentheses refer to the seismometer types listed in Table 1 .

Type 1. (Codes E, L, and 4) Consists of:

a) Geophone - Electrotech EV-17 (E) or Mark Products L4C (L) 1.0 sec. period moving magnet vertical component seismometer or horizontal component adjusted for an output of 0.5 volts/ $\mathrm{cm} / \mathrm{sec}$. and 0.8 critically damped.

b) Preamp/VCO - USGS/OEVE Mode1 J302 or J402 (4) voltage controlled oscillator. Three db points for bandpass filter at $0.1 \mathrm{~Hz}$ and $30 \mathrm{~Hz}$. Signals are transmitted on audio FM carrier over cable or FM radio link to HVO.

Type 3. (Code H1) Consists of:

Electrotech EV-17 or observatory-built $0.8 \mathrm{sec}$. perlod moving coll seismometer with HVO-built solid state seismic preamplifier, galvanometer driver, and $2 \mathrm{~Hz}$ galvanometer. Peak magnification approximately 40,000 at $4 \mathrm{~Hz}$.

Type 4. (Code S) Consists of:

Sprengnether short period vertical and horizontal seismometers (E-W) with 1.5 sec. galvanometers, coupling factor $=0.25,2 \mathrm{X}$ critically damped. Peak magnification approximately $1500 \mathrm{X}$ at $2 \mathrm{~Hz}$.

Type 2 instruments have been discontinued.

Code (W) is a Wood-Anderson torsion seismograph.

Code (MW) is a horizontal component seismograph based on a Type 1 system modified to a Wood-Anderson response.

Codes (TE) and (S5) are experimental seismometers. 


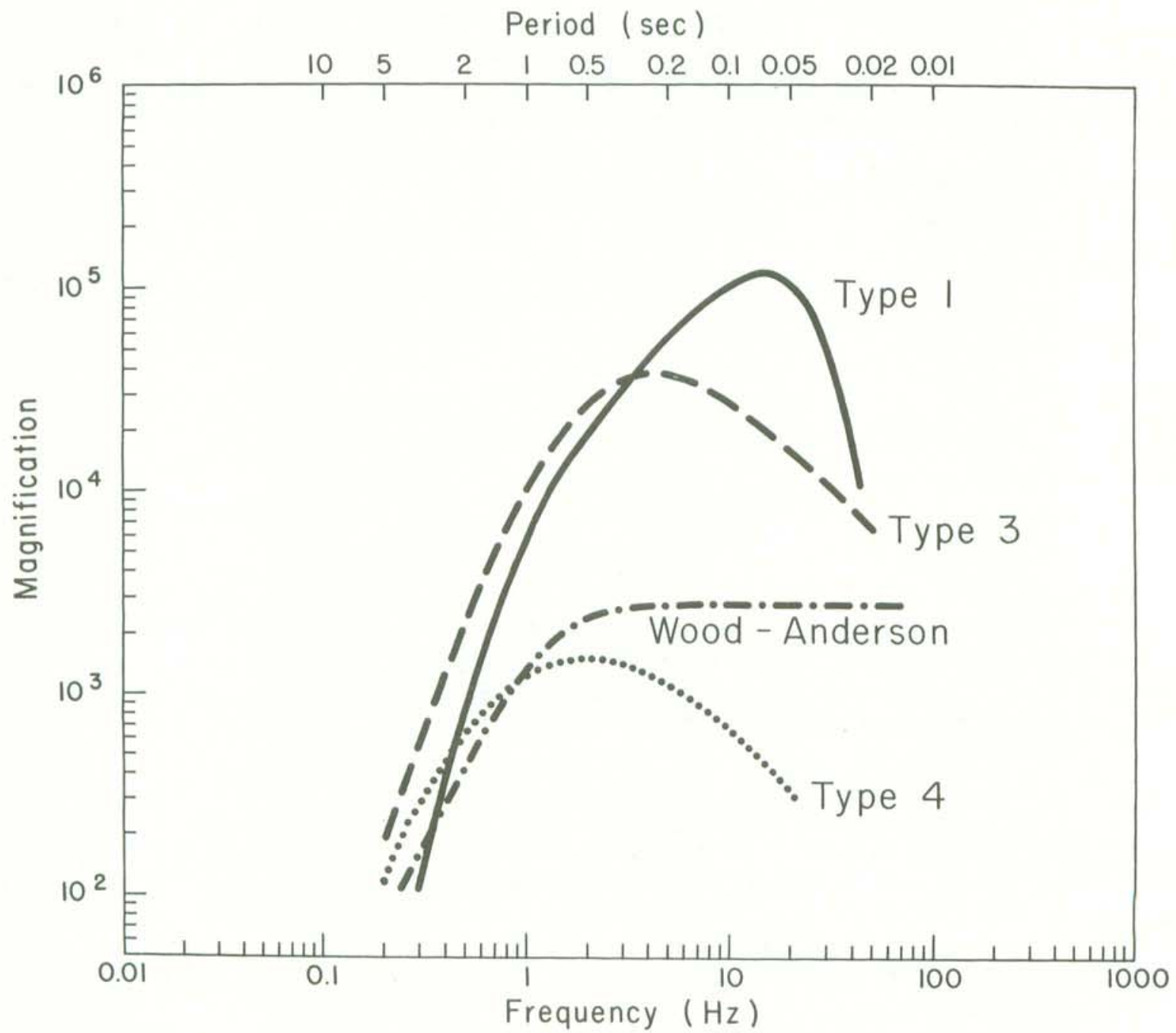

Figure 4. System response curves for the Wood-Anderson torsion seismograph and for the 3 different types of seismometers in use by the Hawallan Volcano observatory. Types 3 and 4 are electro-mechanical seismographs recorded optically on photographic paper. Type 1 is the standard OEVE seismometer recorded on Develocorder film and magnetic tape. The curve for Type 1 includes response of the geophone, all electronics including telemetry, Develocorder galvanometer, and projection of $\mathrm{film}$ by a $20 \mathrm{X}$ viewer. The curves plot the unit response which should be multiplied by a constant but known factor (CAL), to get the reponse for an individual station. 


\section{SEISMIC DATA PROCESSING}

Develocorder films are scanned on a da1ly basis for earthquakes, and coda durations are measured for magnitude determination. Events are digitized, timed, and located on the Eclipse computer at HVO. Computer locations are made using the program HYPOINVERSE (Klein, F.W., Hypocenter location program HYPOINVERSE, U.S.G.S. Open file report 78-694, 1978), and problem events are reread and rerun. Magnetic tape copies of all arrival time and output summary data are kept in Menlo Park and HVO.

The crustal model used is specified by velocities at 4 depth points. Velocity at any depth is given by linear interpolation between points and uses a homogeneous half-space below.

\begin{tabular}{cc}
$\begin{array}{cc}\text { VELOCITY } \\
(\mathrm{km} / \mathrm{sec})\end{array}$ & DEPTH $(\mathrm{km})$ \\
\hline 1.9 & 0.0 \\
6.5 & 4.6 \\
6.9 & 15.0 \\
8.3 & 16.5
\end{tabular}

Two empirical sets of station delays or corrections were used in the locations, and are given in Table 1. Delay model 1 is used for events on Kilauea and 1 ts south flank, and delay model 2 applies to the rest of the island and of fshore earthquakes. The delay models are in fact separated by a circle of radius $34 \mathrm{~km}$ centered at $19^{\circ} 22^{\prime} \mathrm{N}$ and $155^{\circ} 10^{\prime} \mathrm{W}$.

Magnitudes for most events were computed using both recorded amplitudes on low gain or Wood-Anderson stations, and signal or coda duration on selected short-period vertical stations. Amplitudes read from other than Wood-Anderson instruments are corrected to an equivalent Wood-Anderson amplitude using the curves of Figure 3 and CAL factors. Amplitude magnitudes larger than 2.5 are generally based on the Wood-Anderson instruments in Hilo or Type 4 seismographs at Uwekahuna. Smaller events may occasionally include amplitude readings from stations AHU, OTL, PPL, KHU, or WIL.

Duration magnitudes are determined from the length of signal in seconds read from the Develocorder viewer. This time, also called the "F-P time" is measured from the first $P$ arrival to the point where the earthquake signal has decayed nearly to the noise level. A bilinear relation is an appropriate fit to the data sample and is used to compute all duration magnitudes. Duration times are only read from Type 1 selsmographs. Because duration magnitudes are relatively insensitive to station response and can be determined using the high-gain short-period stations, it is felt that duration magnitudes are more accurate and complete at the lower magnitudes (below 2).

The equations used in magnitude determination are:

$$
\begin{array}{lll}
\text { duration }<210 \mathrm{sec} & M=-5.2+3.89 \log (\mathrm{F}-\mathrm{P})+.013 \mathrm{z}+.0037 \mathrm{D} \\
\text { duration }>210 \mathrm{sec} & M=-.905+2.026 \log (\mathrm{F}-\mathrm{P})+.013 \mathrm{z}+.0037 \mathrm{D}
\end{array}
$$

where $z$ and $D$ are the depth and epicentral distance $\mathrm{in} \mathrm{km}$, respectively. 


\section{SEISMIC SUMMARY}

The emphasis in both station coverage and detailed data analysis is on the highly active south half of the Island of Hawail. Hundreds of earthquakes too small to locate are classified generally and counted dally. The set of wellrecorded earthquakes located in the Hawail Island region is nearly complete above magnitude 2.0. Many smaller events are located in the densely instrumented Kilauea area. Substantial effort is made to locate earthquakes elsewhere within the Hawailan Archipelago. Such coverage cannot be as complete as on the south flank, but nearly all events above magnitude 4.0 are located with limited precision.

Data presented in the seismic summary is in four parts. Table 3 gives duration of harmonic tremor and numbers of earthquakes (most too small to locate) from several source reglons around Kilauea and Mauna Loa. The source region is determined visually from signal character and pattern of arrival times at key stations. Maps showing computer located hypocenters are given in Figures 9-22. The location maps are of different scales and provide hypocenters with magnitude thresholds set at $1.0,2.0,3.0$ and 3.5 varied according to region.

The list of computer locations constitutes the bulk of this summary, and is given in Table 5. Each earthquake in the list is assigned a three-letter code based on 1 ts location and depth. Figures 5-8 are maps of the regions used to assign the location codes. The latitude and longitude $11 \mathrm{mits}$ of rectangular regions are listed in Table 4. When the listed coordinates imply an overlap, precedence is given according to Figures 5-8. Table 6 relists the events in Table 5 for which either duration or amplitude magnitude is 3.0 or larger. This list includes many of the earthquakes felt in Hawail.

Table 3. Number of earthquakes and minutes of tremor recorded on seismographs around Kilauea and Mauna Loa.

Earthquake categories are:

1) Kilauea summit, short period caldera: shallow earthquakes beneath the caldera.

2) Kilauea summit, long period caldera: earthquakes characterized by low frequency signatures, of ten originating $5-10 \mathrm{~km}$ below the caldera.

3) Kilauea summit $30 \mathrm{~km}$ : deep earthquakes about $30 \mathrm{~km}$ beneath the summit region.

4) Kaoik1 and southwest rift: earthquakes beneath the southwest rift of Kilauea, western parts of the Koae faults and adjacent Kaolki fault system.

5) Upper east rift: earthquakes in the upper and middle east rift zone, the adjacent parts of the south flank, and eastern parts of the Koae faults.

6) Lower east rift: earthquakes in the lower east rift zone and adjacent parts of the south flank.

7) Offshore PPL: earthquakes from offshore areas south of the Puu Pili station, including Loihi seamount.

8) Mauna Loa long period: low frequency events near Mauna Loa summit.

9) Mauna Loa short period: shallow earthquakes in the Mauna Loa caldera region.

Tremor is separated into four categories: shallow, intermediate and deep Kilauea, and Mauna Loa. Depth is inferred on the basis of relative amplitudes on seismographs. 
RILAUEA SUMMIT

KILAUEA FLANK

MAUNA LOA TREMOR (MINUTES)

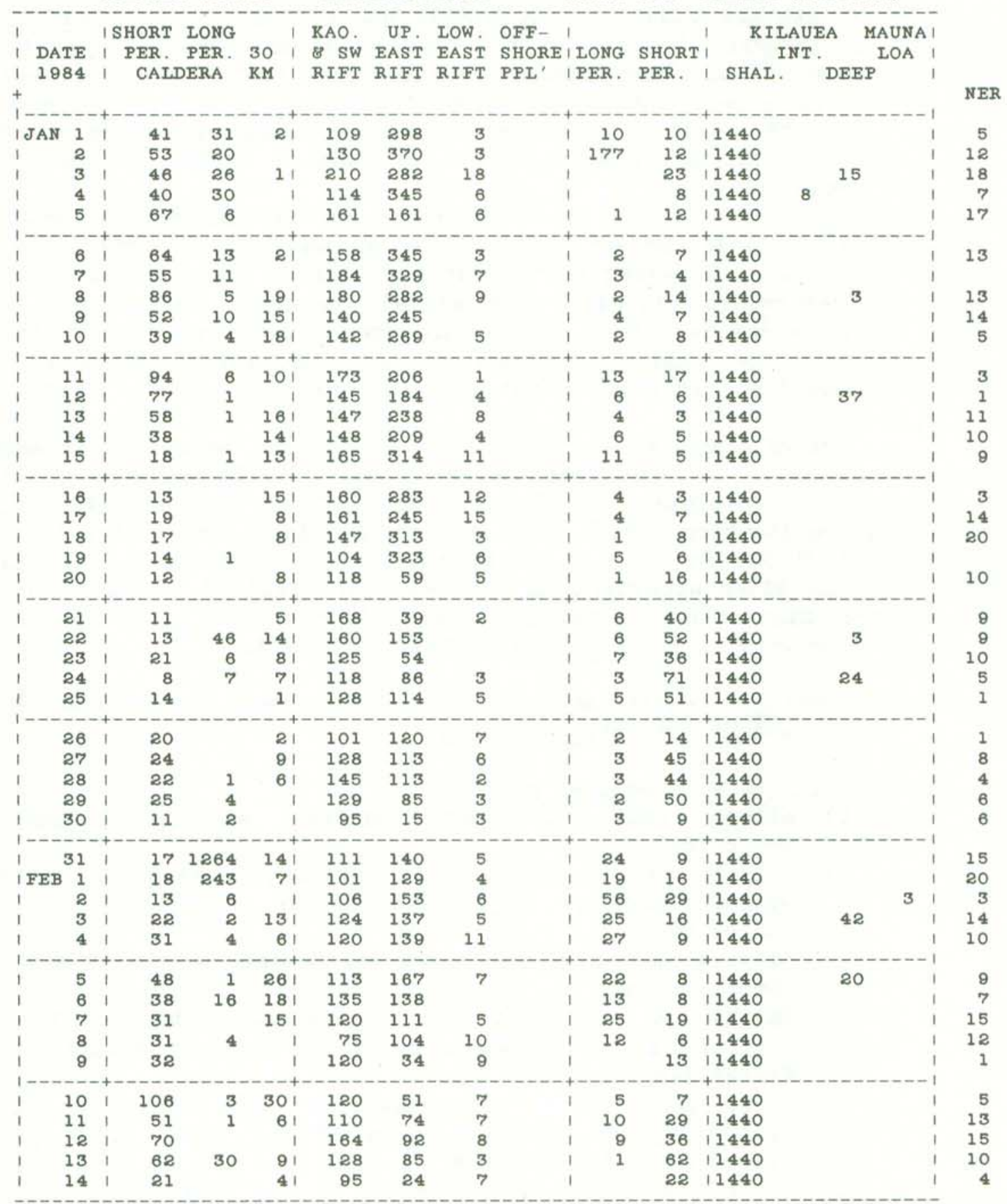




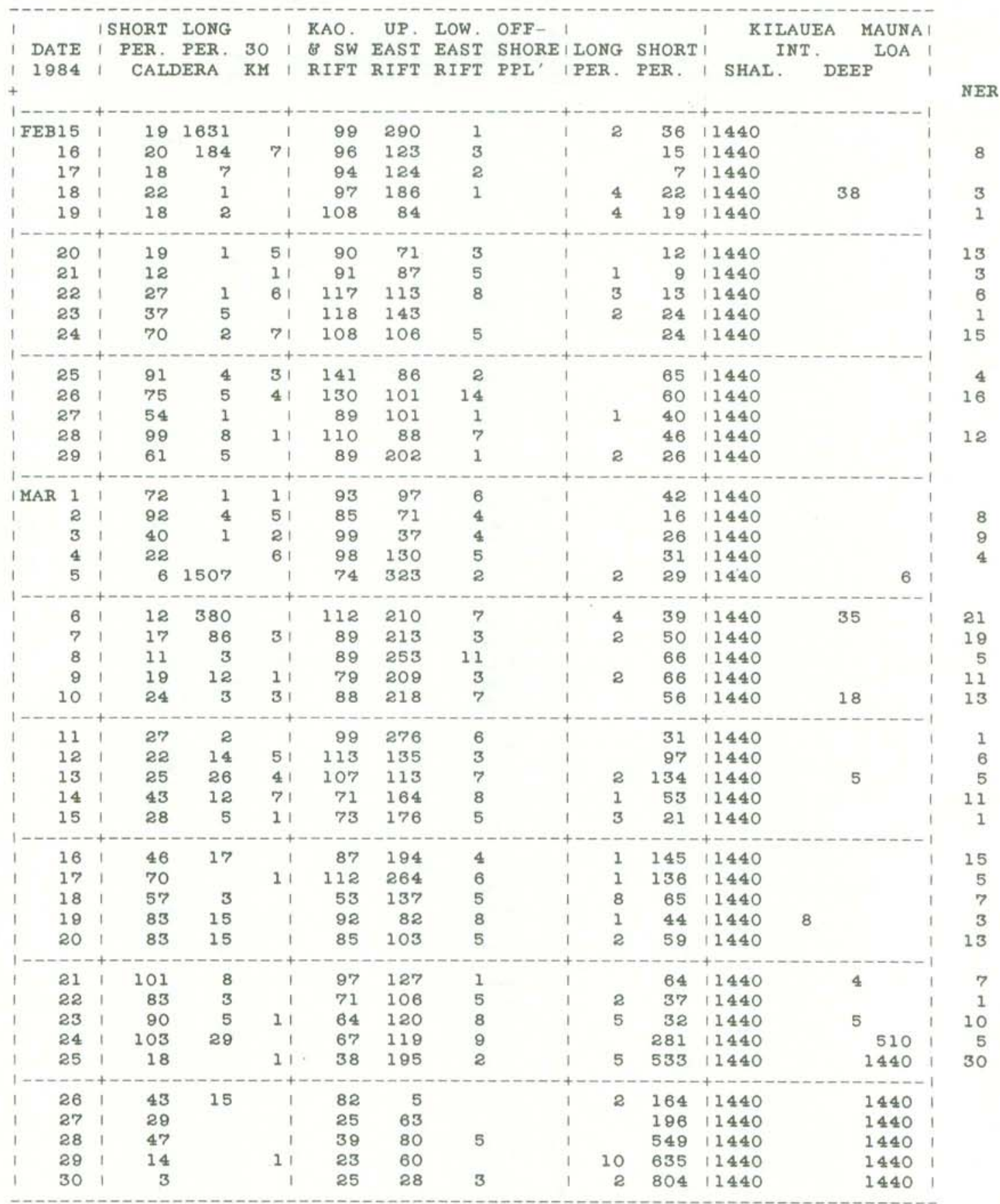


RILAUEA SUMMIT KILAUEA FLANK MAUNA LOA TREMOR (MINUTES)

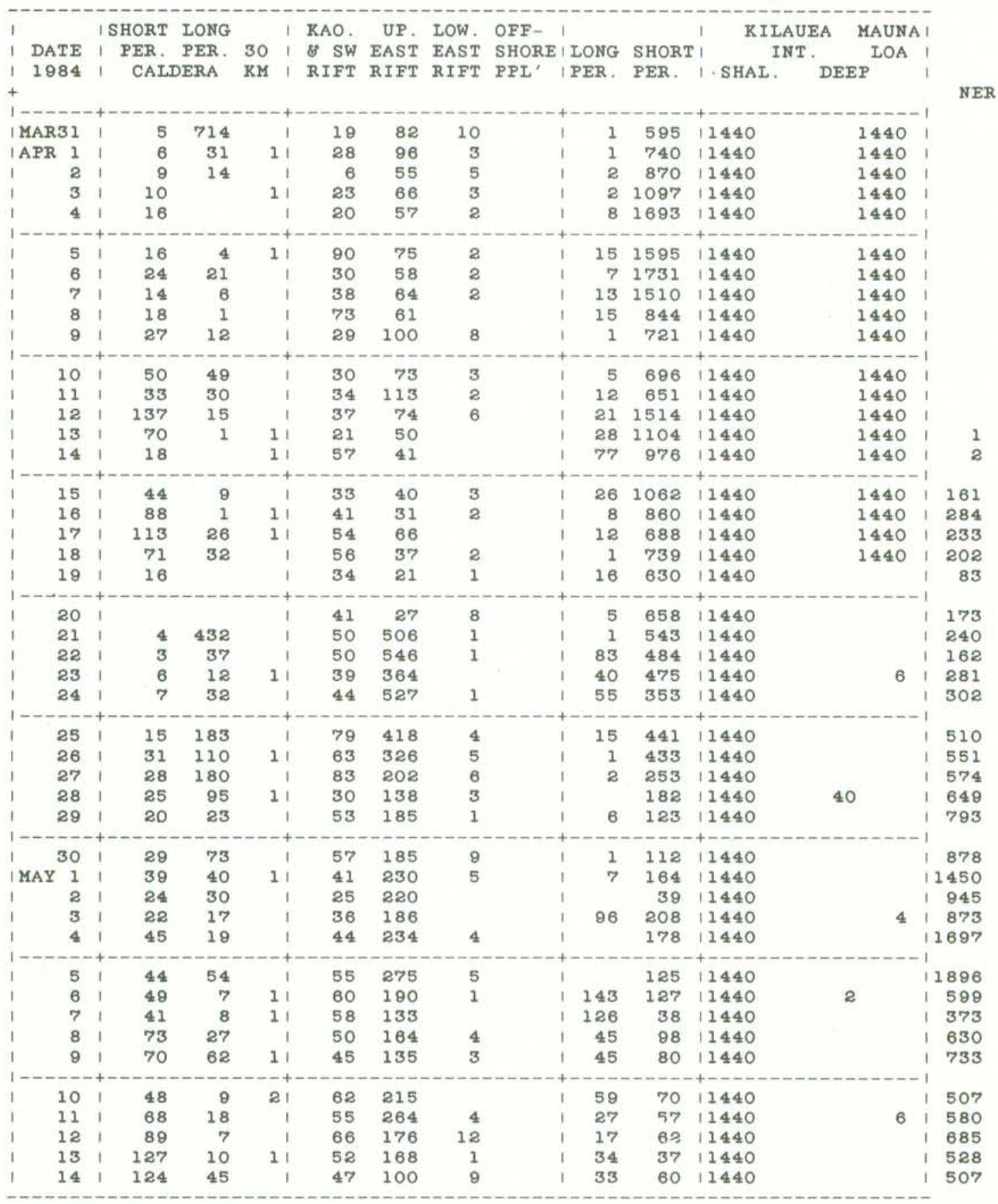




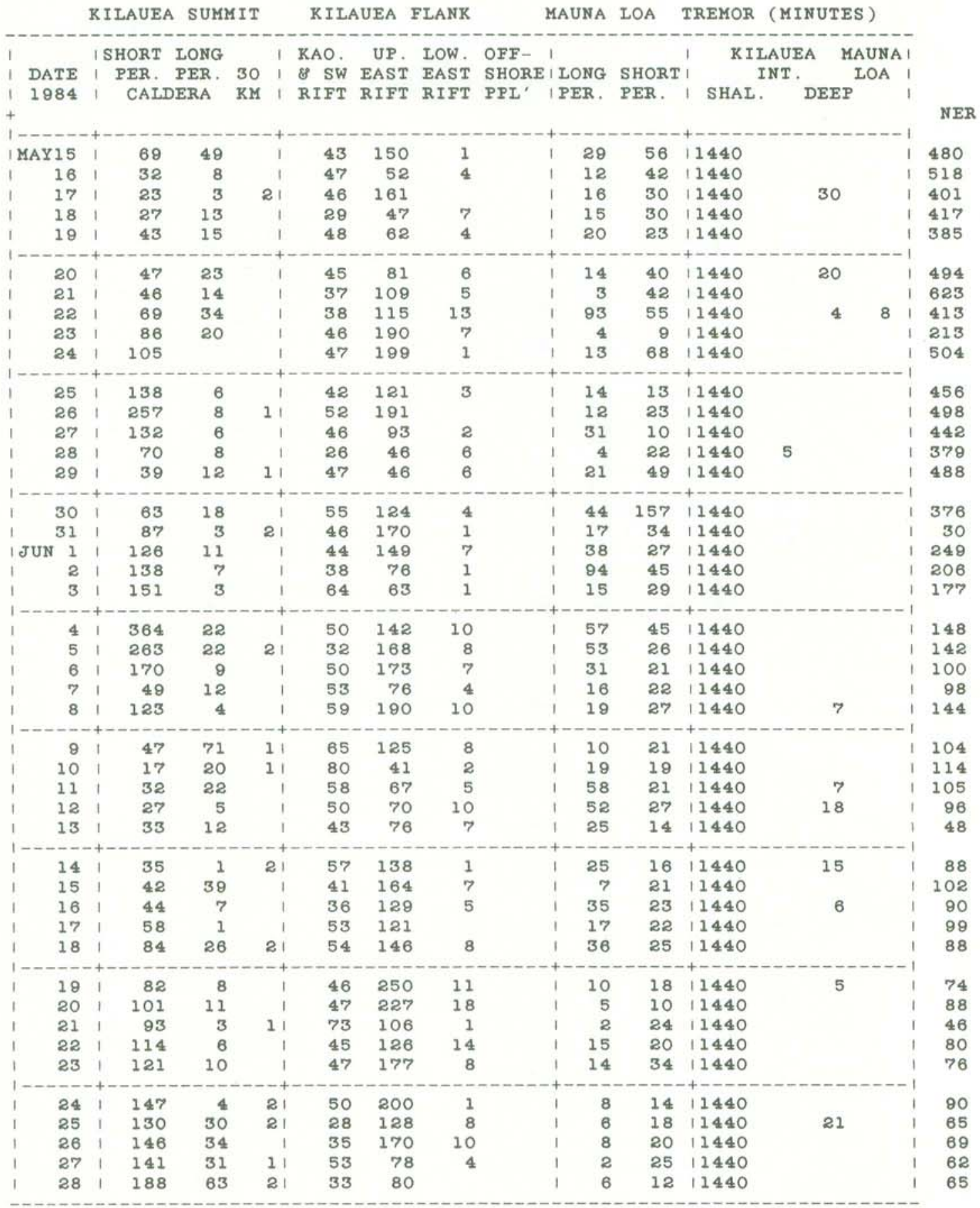




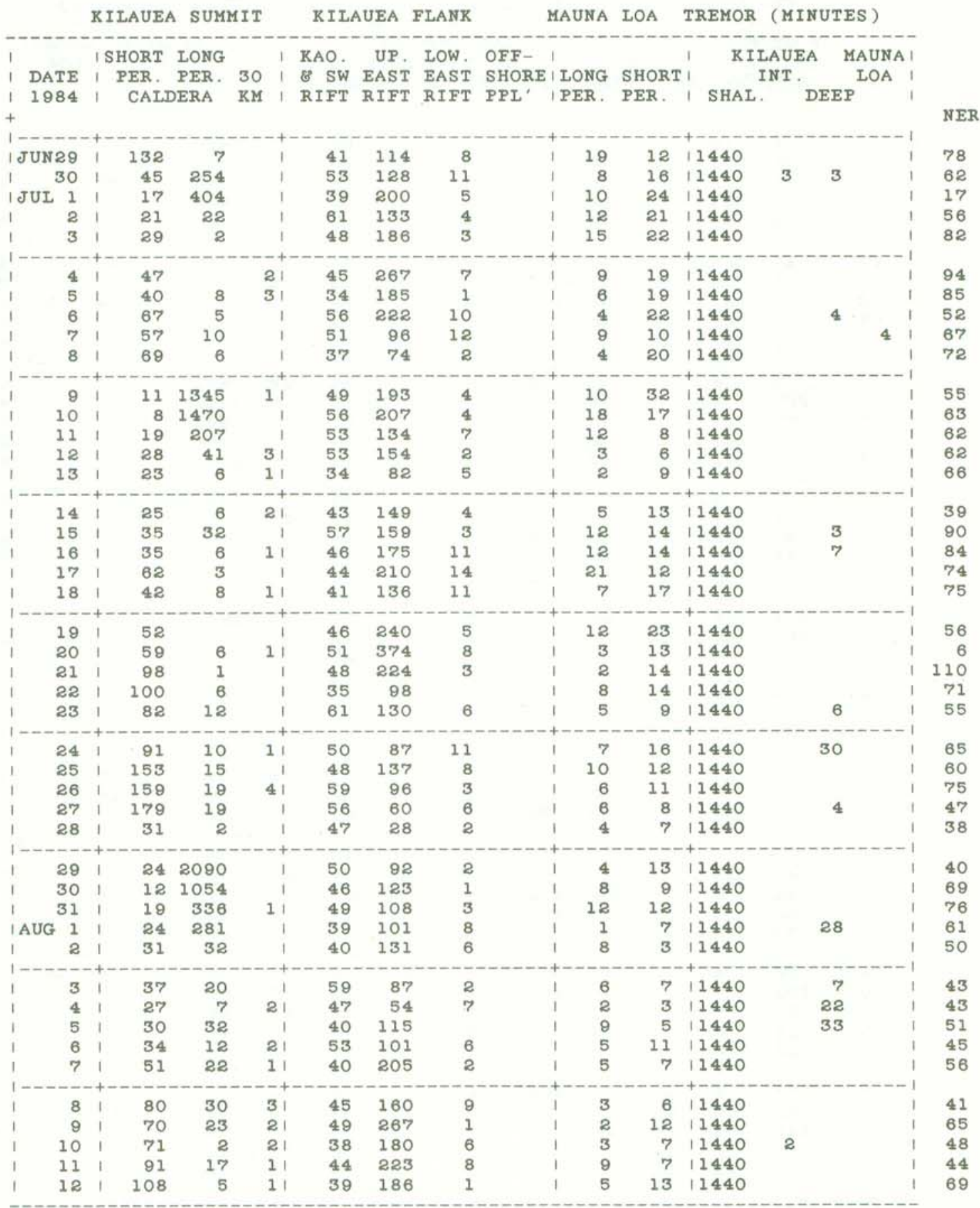




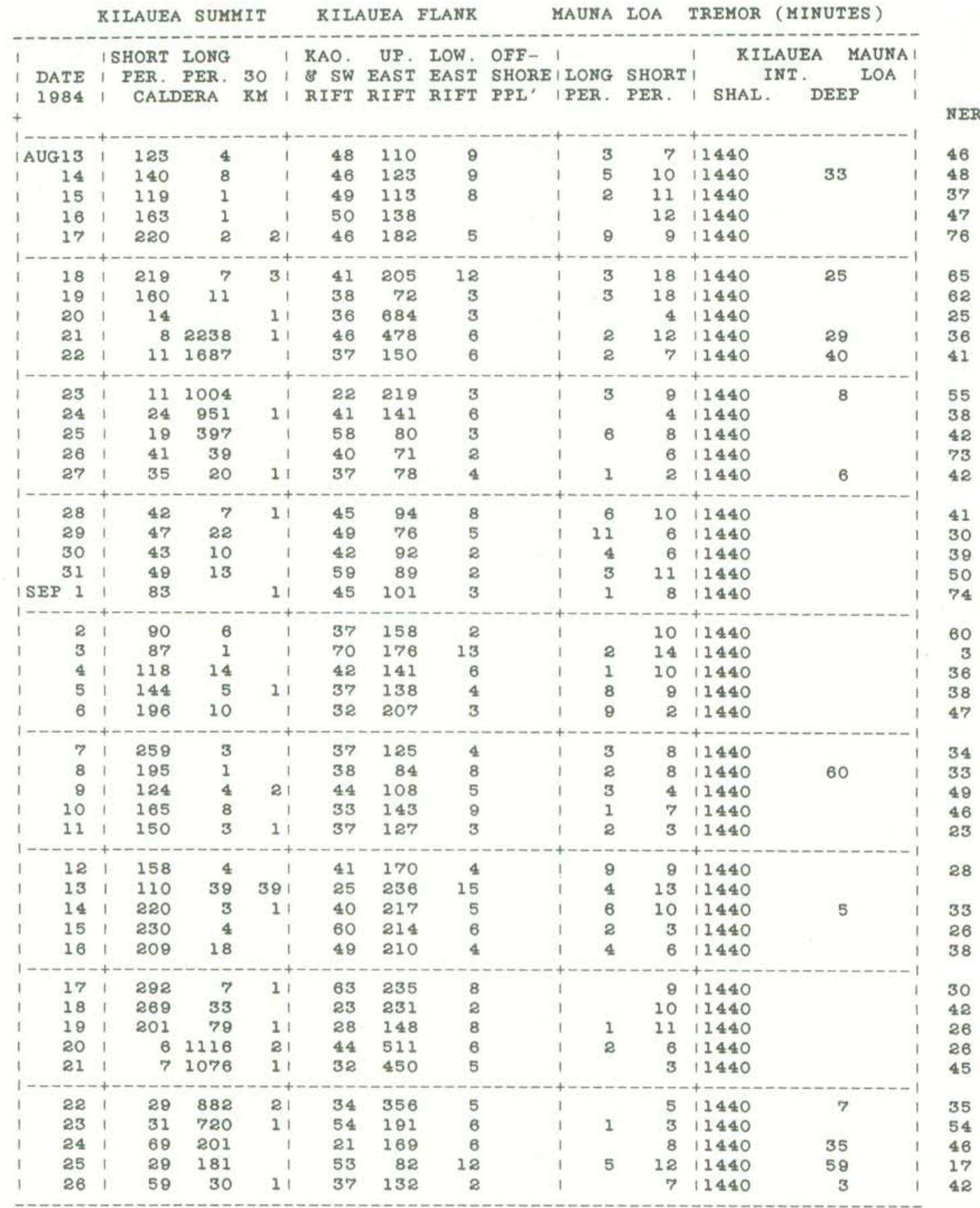


KILAUEA SUMMIT

KILAUEA FLANK

MAUNA LOA TREMOR (MINUTES)

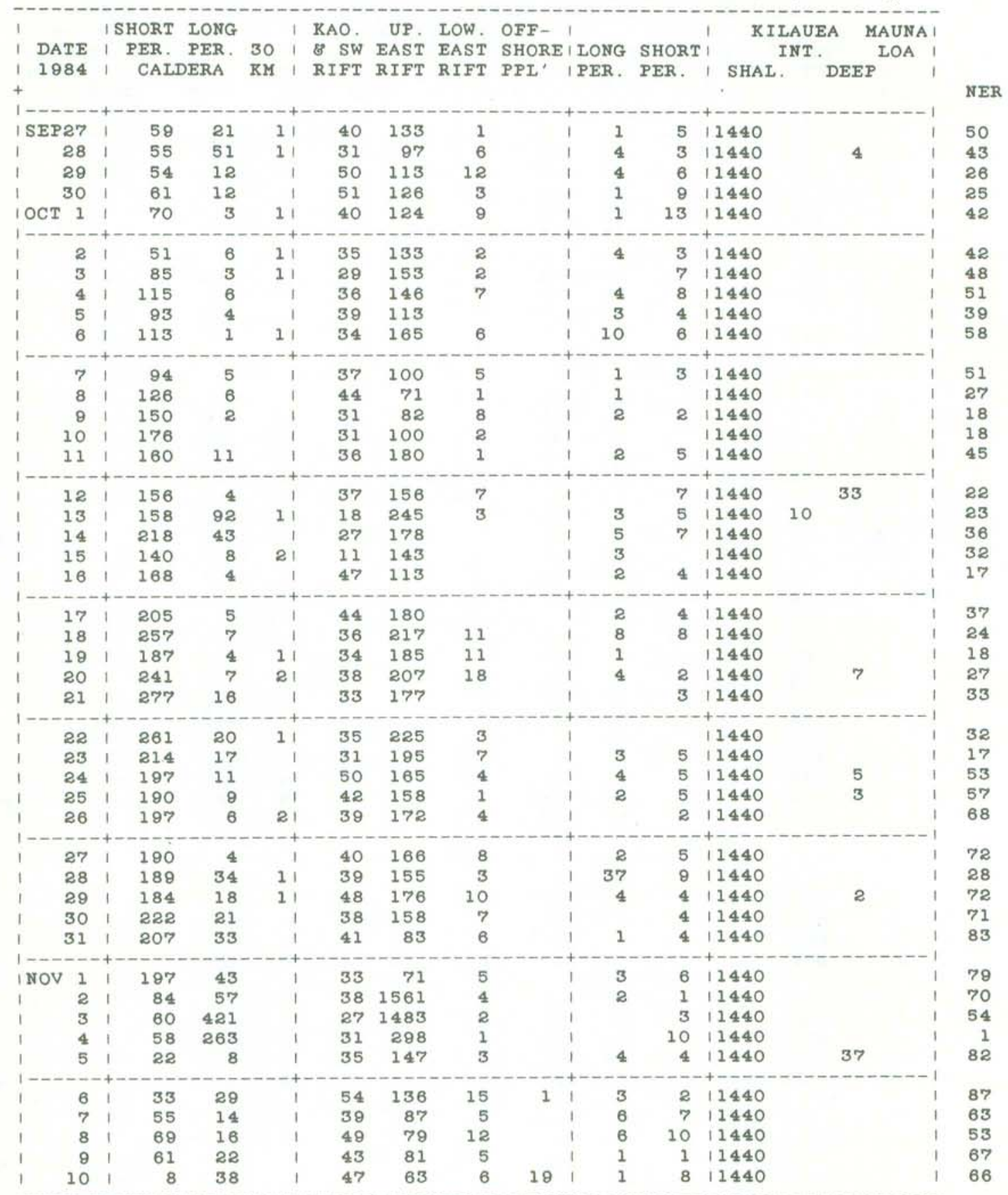




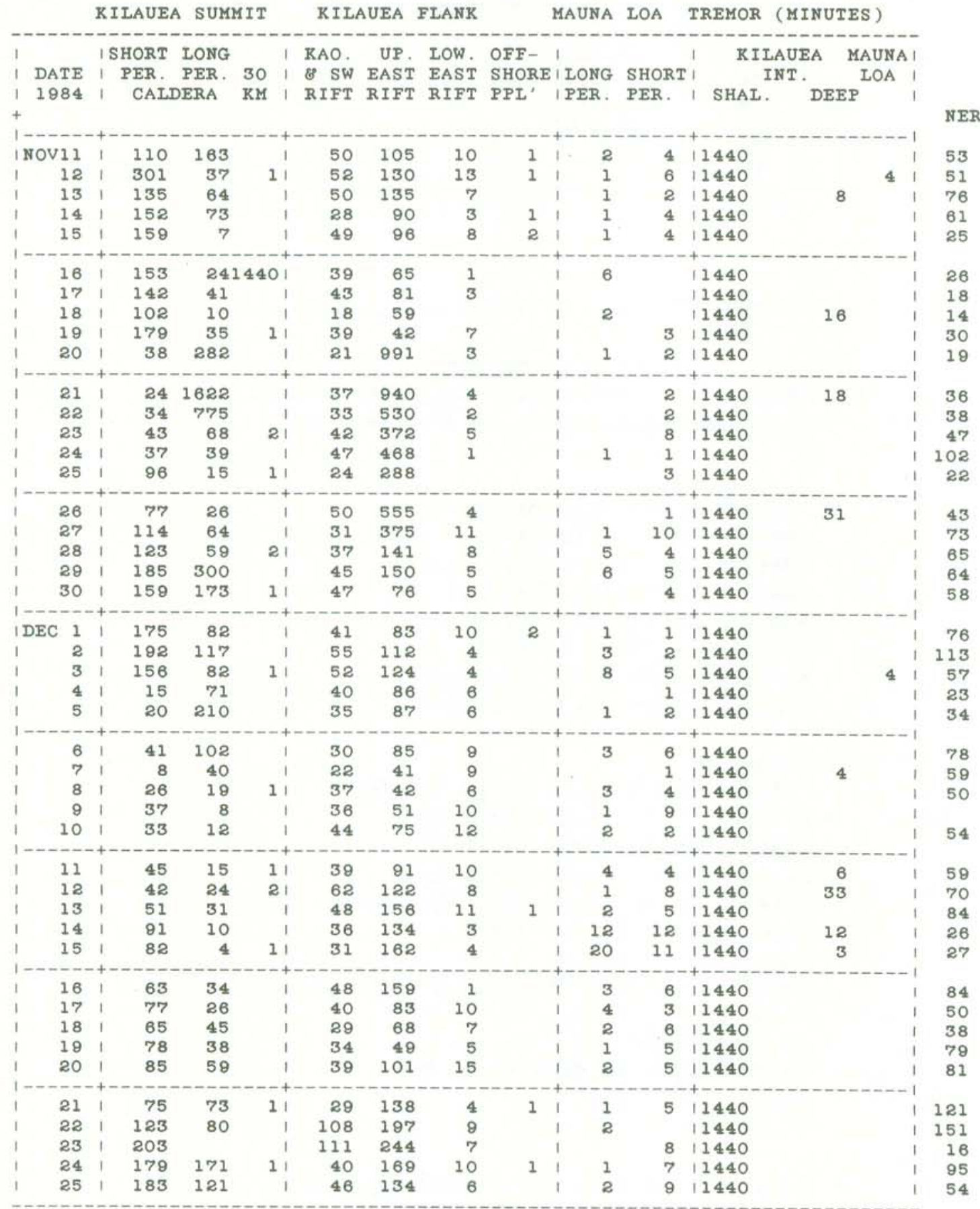




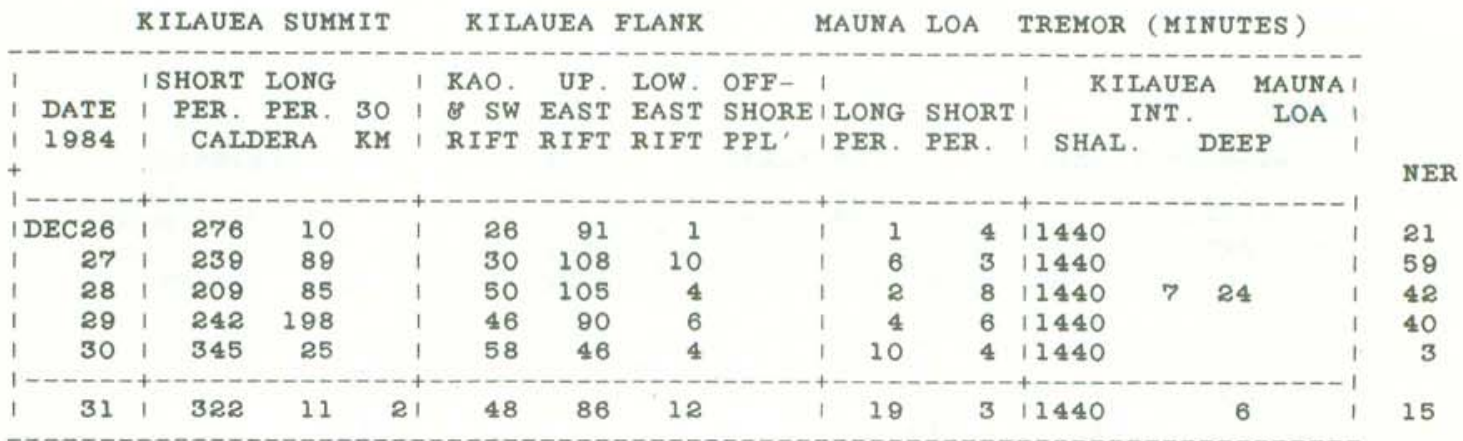

Table 4. Coordinates of named earthquake regions.

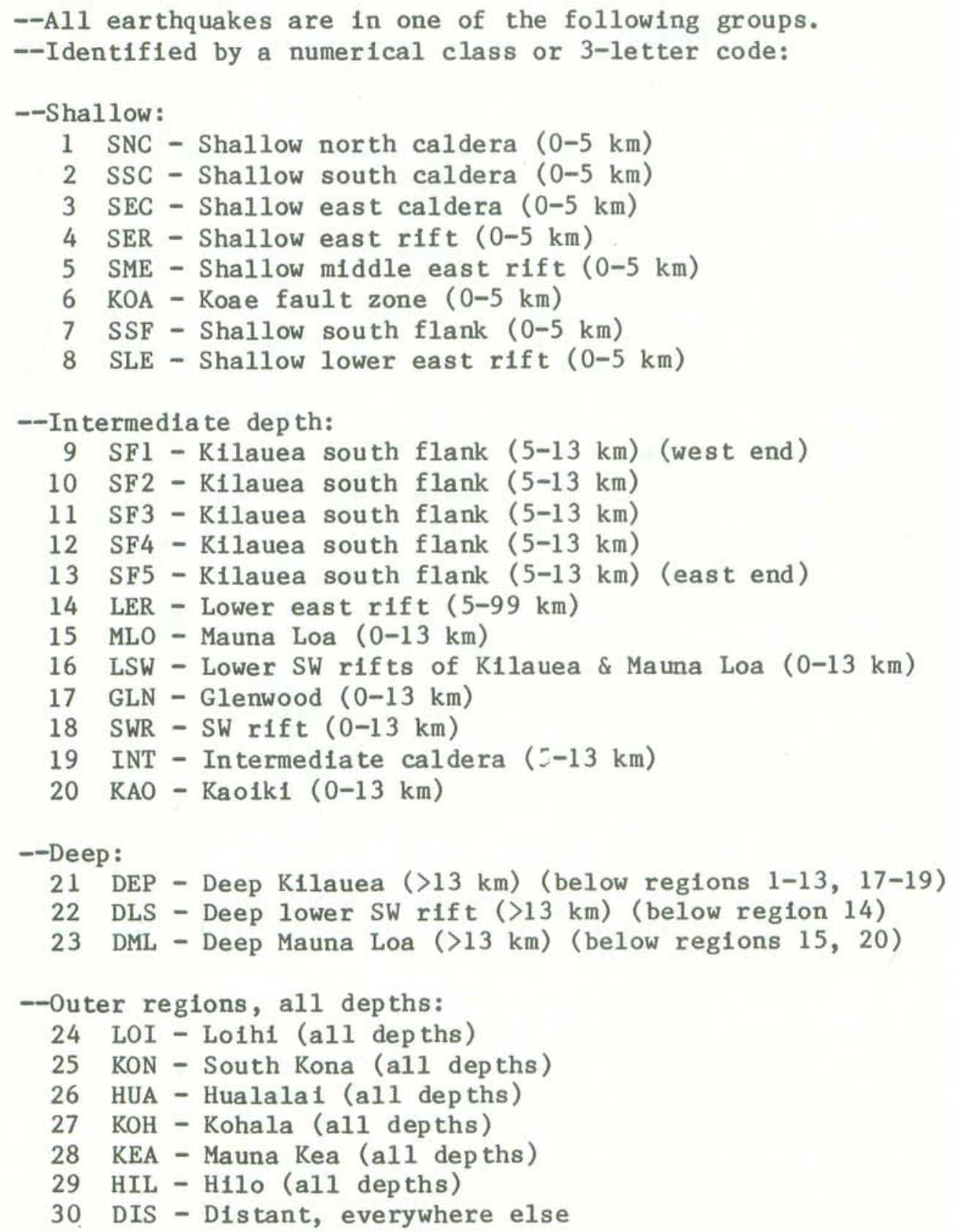


Table 4 (continued)

--The latitude and longitude limits of the regions are given below.

--When the coordinates imply an overlap, precedence is given as in the maps.

\begin{tabular}{|c|c|c|c|c|}
\hline No. & Code & N. Lat. & S.Lat. & W. Lon. \\
\hline 1 & SNC & 1928 & 1924.5 & 15519 \\
\hline 2 & SSC & 1924.5 & 1922 & 15519 \\
\hline 3 & SEC & 1924.5 & 1922 & 15516.5 \\
\hline 4 & SER & 1926 & 1920.5 & $155 \quad 14$ \\
\hline 5 & SME & 1926 & --- & 15507.2 \\
\hline 6 & KOA & 1922 & 1920.5 & $\begin{array}{ll}155 & 17\end{array}$ \\
\hline 7 & SSF & -- & 1910 & $\begin{array}{lll}155 & 17\end{array}$ \\
\hline 8 & SLE & 1932 & 1916 & 15500 \\
\hline 9 & SF1 & 1922 & 1910 & $\begin{array}{lll}155 & 17\end{array}$ \\
\hline 10 & SF2 & 1926 & 1910 & 15514.5 \\
\hline 11 & SF3 & 1926 & 1910 & 15512.3 \\
\hline 12 & SF4 & 1926 & 1910 & $\begin{array}{lll}155 & 09.1\end{array}$ \\
\hline 13 & SF5 & 1926 & 1910 & 15505.3 \\
\hline 14 & LER & 1932 & 1916 & 15500 \\
\hline 15 & MLO & 1935 & 1919 & 15535 \\
\hline 16 & LSW & $19 \quad 19$ & 1840 & 15543 \\
\hline 17 & GLN & 1935 & 1926 & $155 \quad 19$ \\
\hline 18 & SWR & 1922 & 1910 & 15525 \\
\hline 19 & INT & 1928 & 1922 & $\begin{array}{ll}155 & 19\end{array}$ \\
\hline 20 & KAO & 1930 & 1919 & $155 \quad 32$ \\
\hline 21 & DEP & 1935 & 1910 & 15525 \\
\hline 22 & DLS & 1919 & 1840 & $155 \quad 43$ \\
\hline 23 & DML & 1935 & 1919 & $155 \quad 35$ \\
\hline 24 & LOI & 1910 & 1840 & 15525 \\
\hline 25 & KON & 1939 & 1900 & 15620 \\
\hline 26 & HUA & 1955 & 1939 & 15620 \\
\hline 27 & $\mathrm{KOH}$ & 2025 & 1955 & 15620 \\
\hline 28 & KEA & 2025 & 1935 & 15534 \\
\hline 29 & HIL & 1947 & 1932 & 15509 \\
\hline
\end{tabular}

E. Lon. 15514

15516.5

15514

15507.2

15500

15514

15500

15440

15514.5

$\begin{array}{lll}155 & 12.3\end{array}$

$\begin{array}{lll}155 & 09.1\end{array}$

$\begin{array}{lll}155 & 05.3\end{array}$

15500

15440

15519

15525

15500

$\begin{array}{lll}155 & 17\end{array}$

15514

$155 \quad 19$

15500

15525

15519

15500

15543

15543

15534

15440

15440 
Figure 5. Earthquake classification, shallow $0-5 \mathrm{~km}$ deep Kilauea and east flank Mauna Loa.

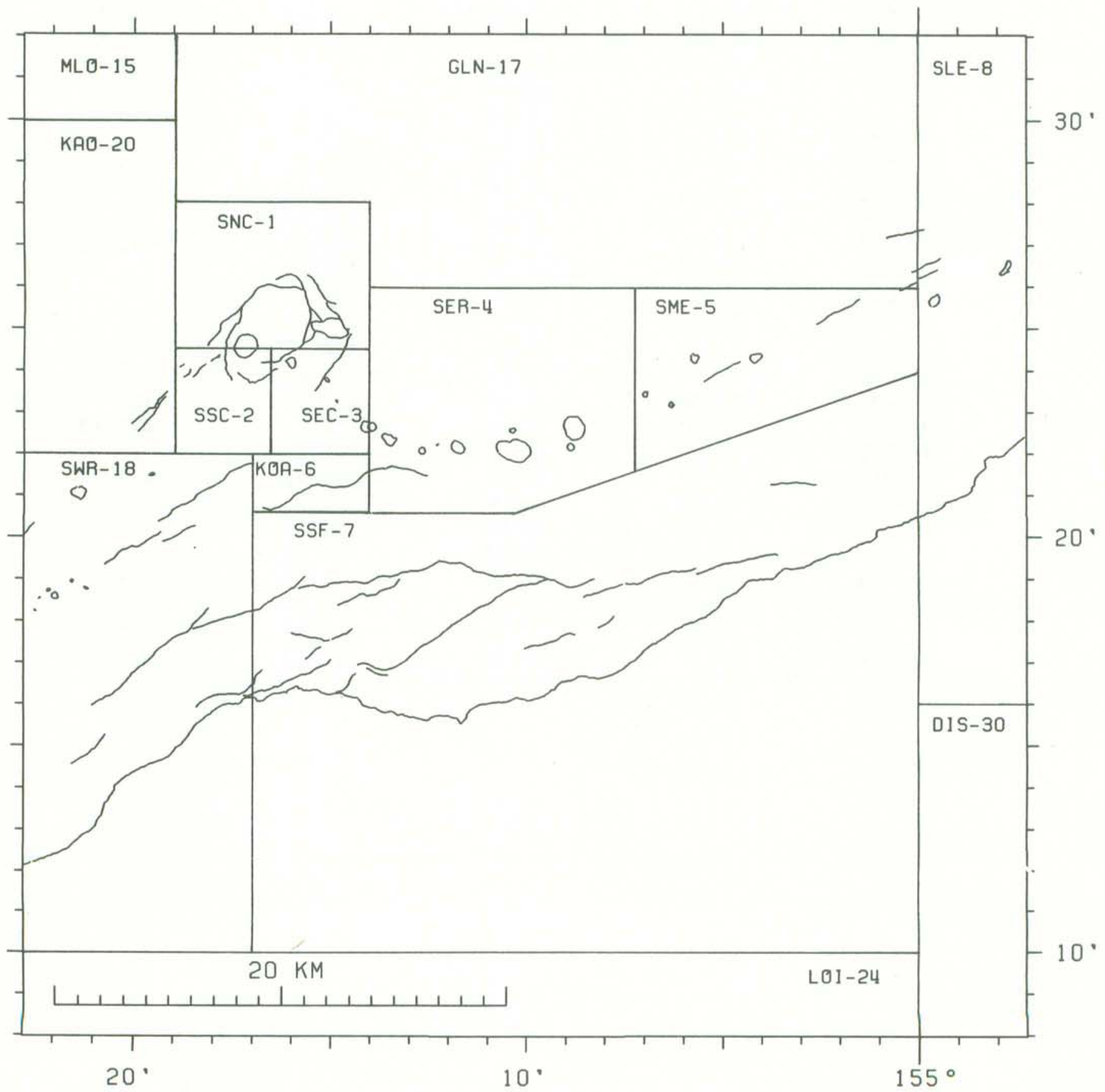


Figure 6. Earthquake classification, intermediate 5 - $13 \mathrm{~km}$ deep Kilauea and east flank Mauna Loa.

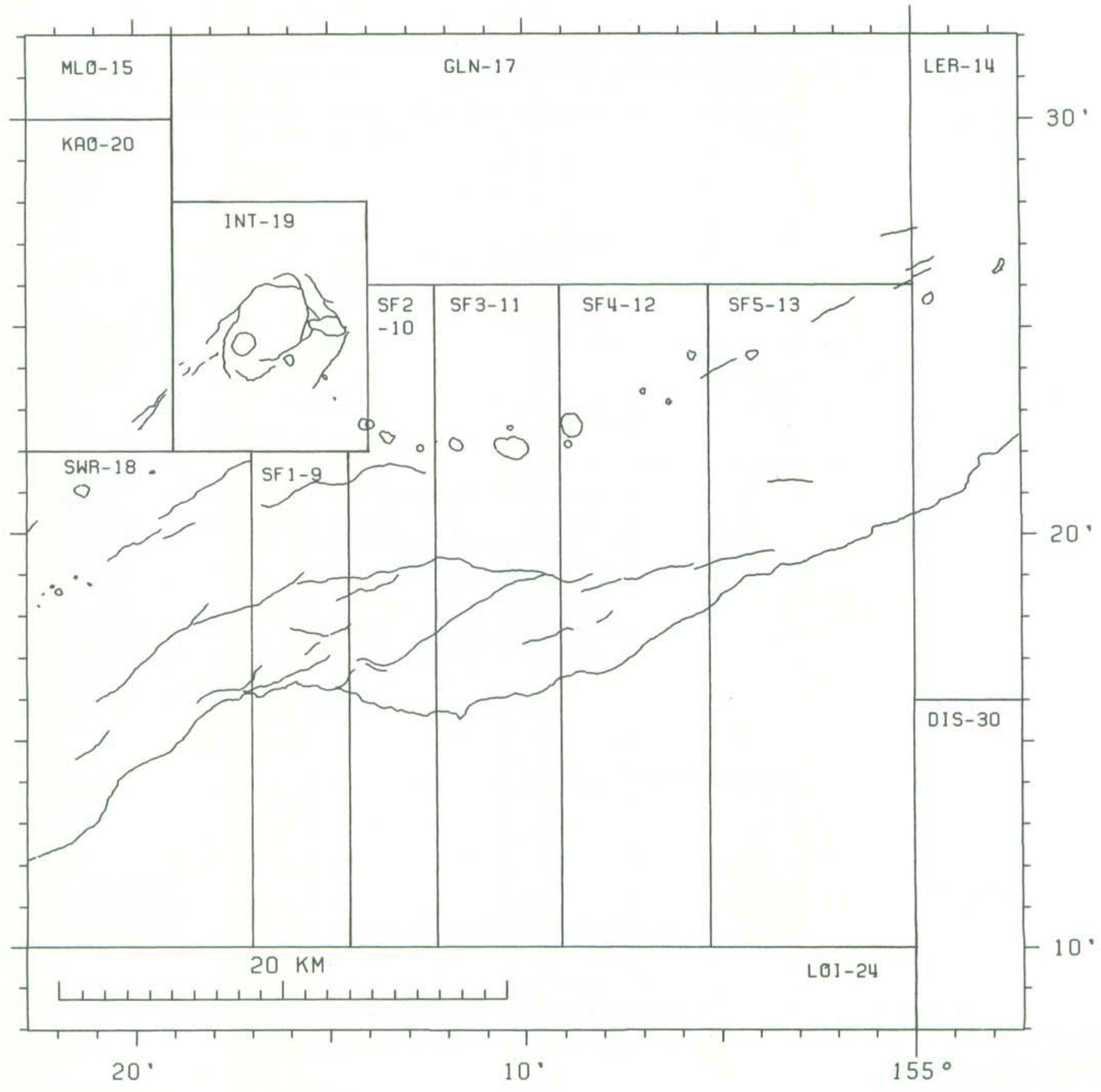


Figure 7. Earthquake classification, crustal $0-13 \mathrm{~km}$ deep island of Hawaii.

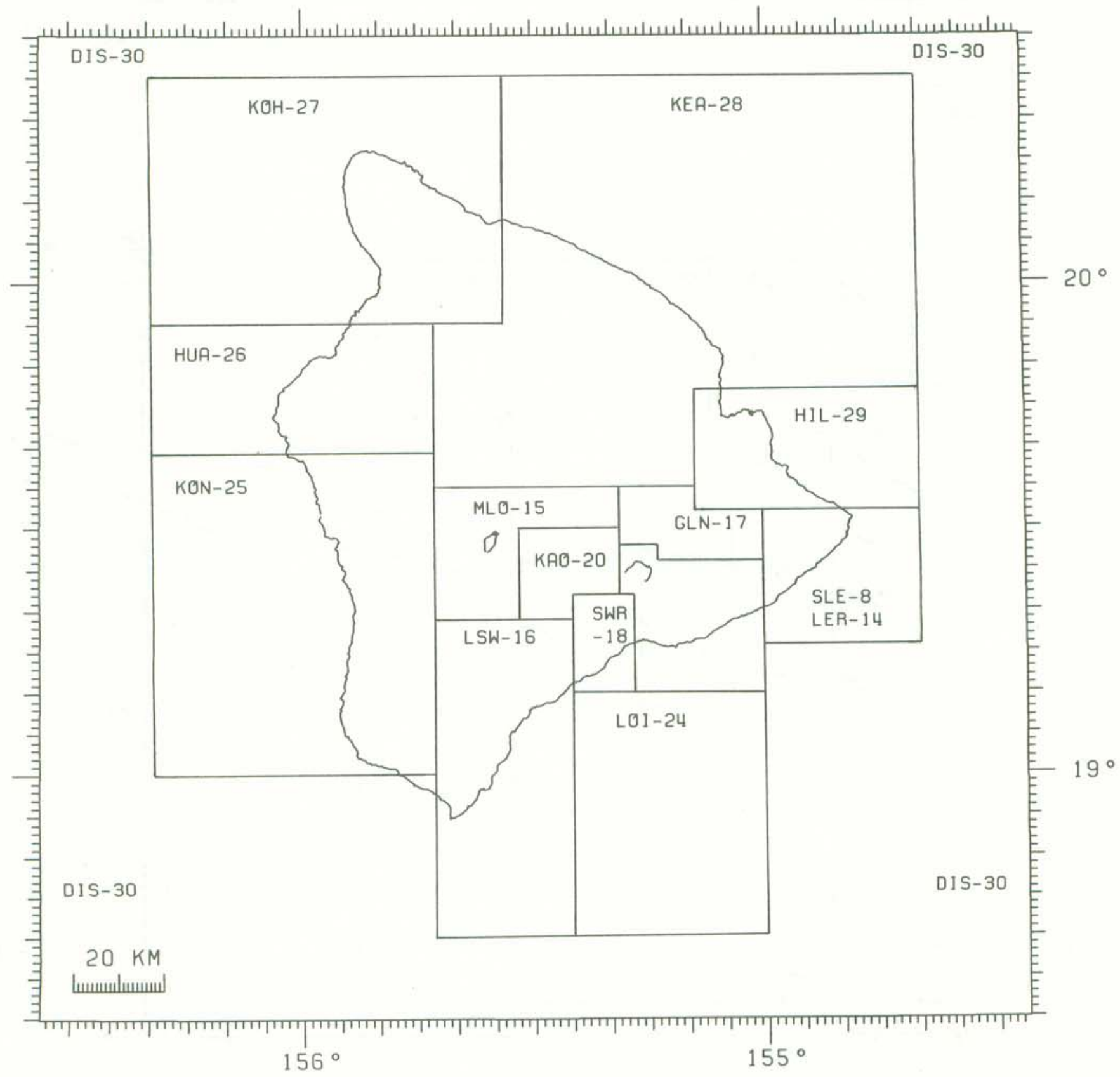


Figure 8. Earthquake classification, mantle greater than $13 \mathrm{~km}$ deep island of Hawaii.

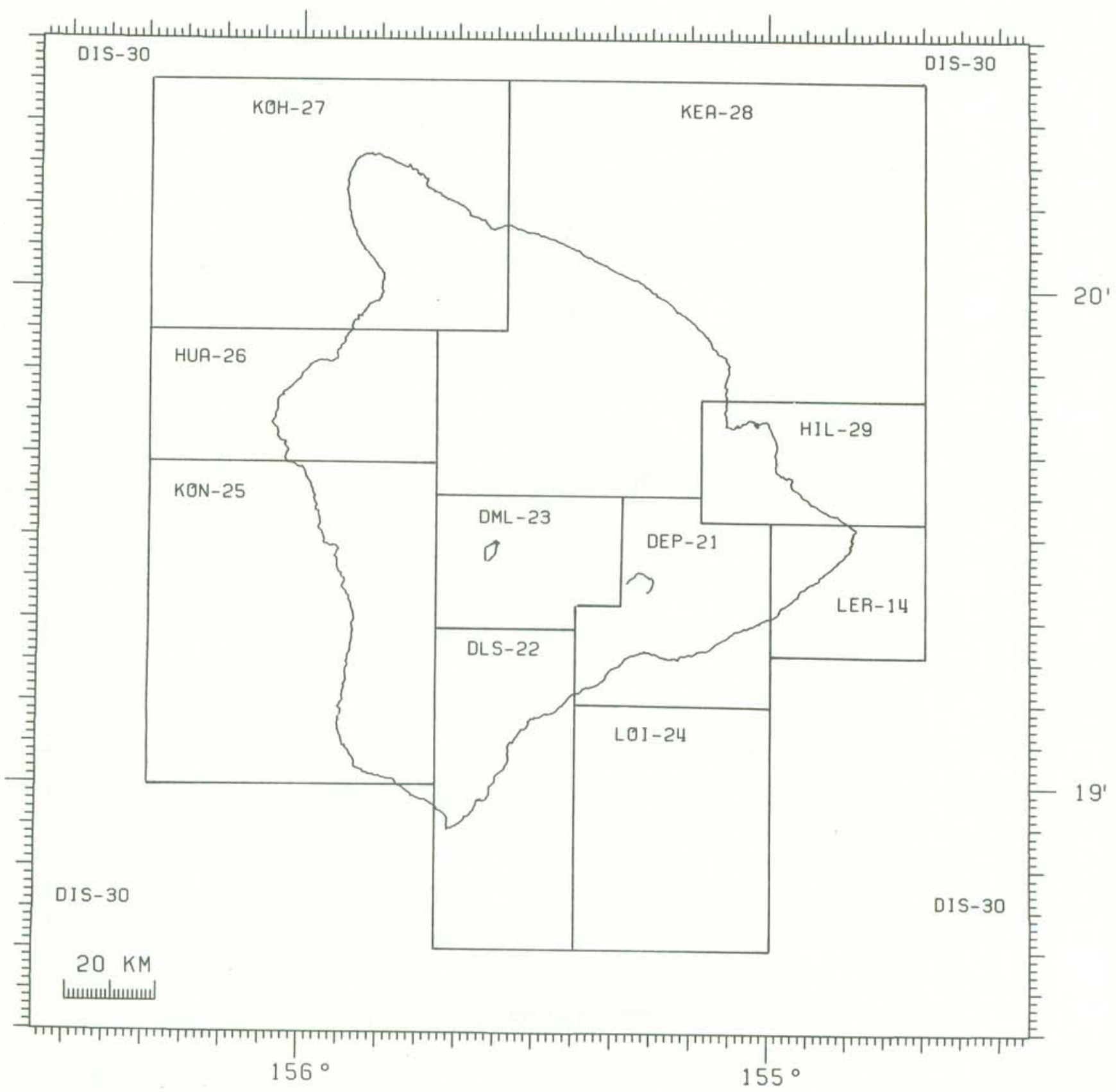


Figure 9

1984 HAWAIIAN ISLANDS EARTHQUAKE LOCATIONS

0-60 KM DEPTHS, $M>=3.5$

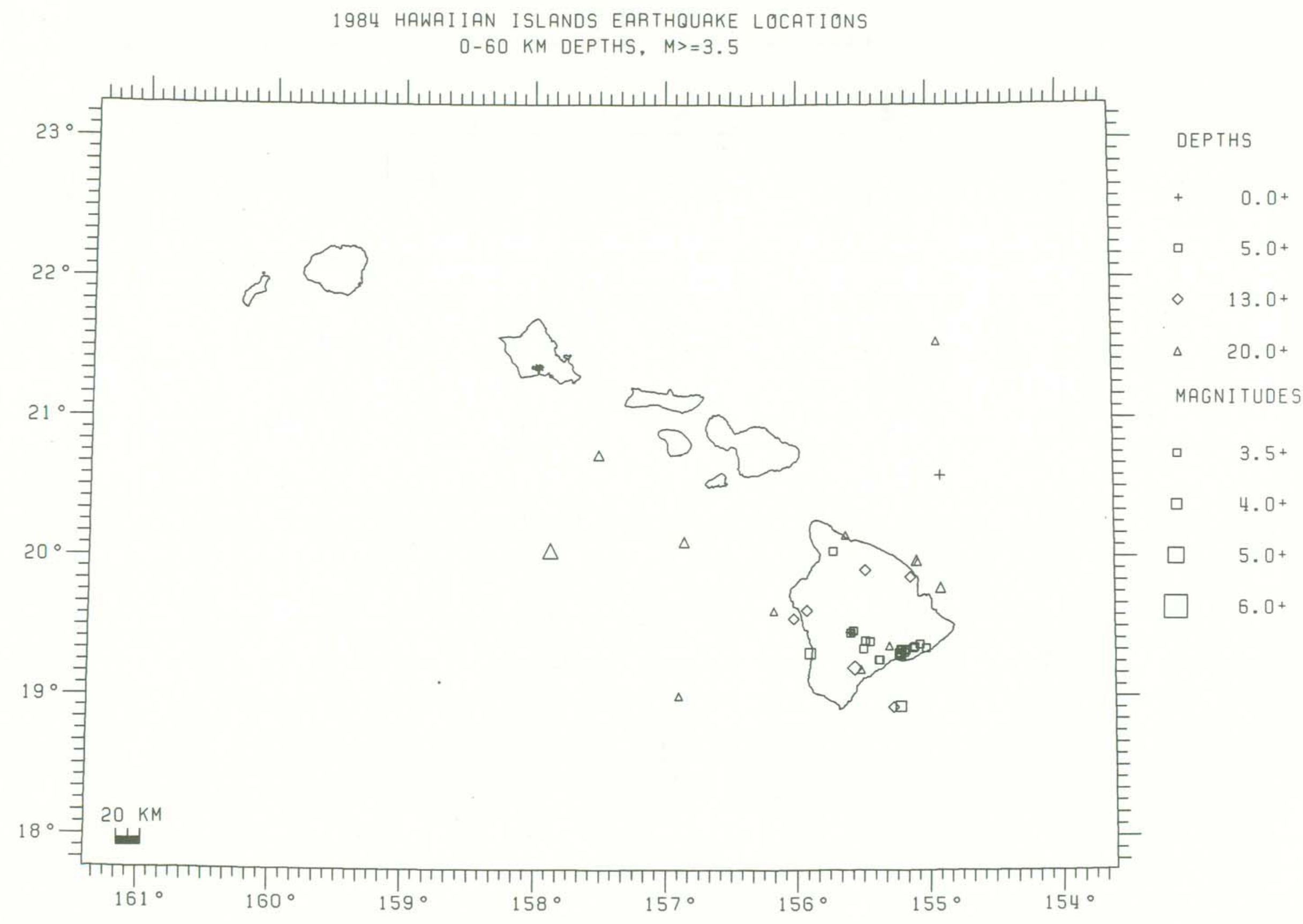


Figure 10

$$
\begin{aligned}
& 1984 \text { HAWAII ISLAND EARTHQUAKE LOCATIONS } \\
& 0-5.0 \mathrm{KM} \text { DEPTHS, M>=2 }
\end{aligned}
$$

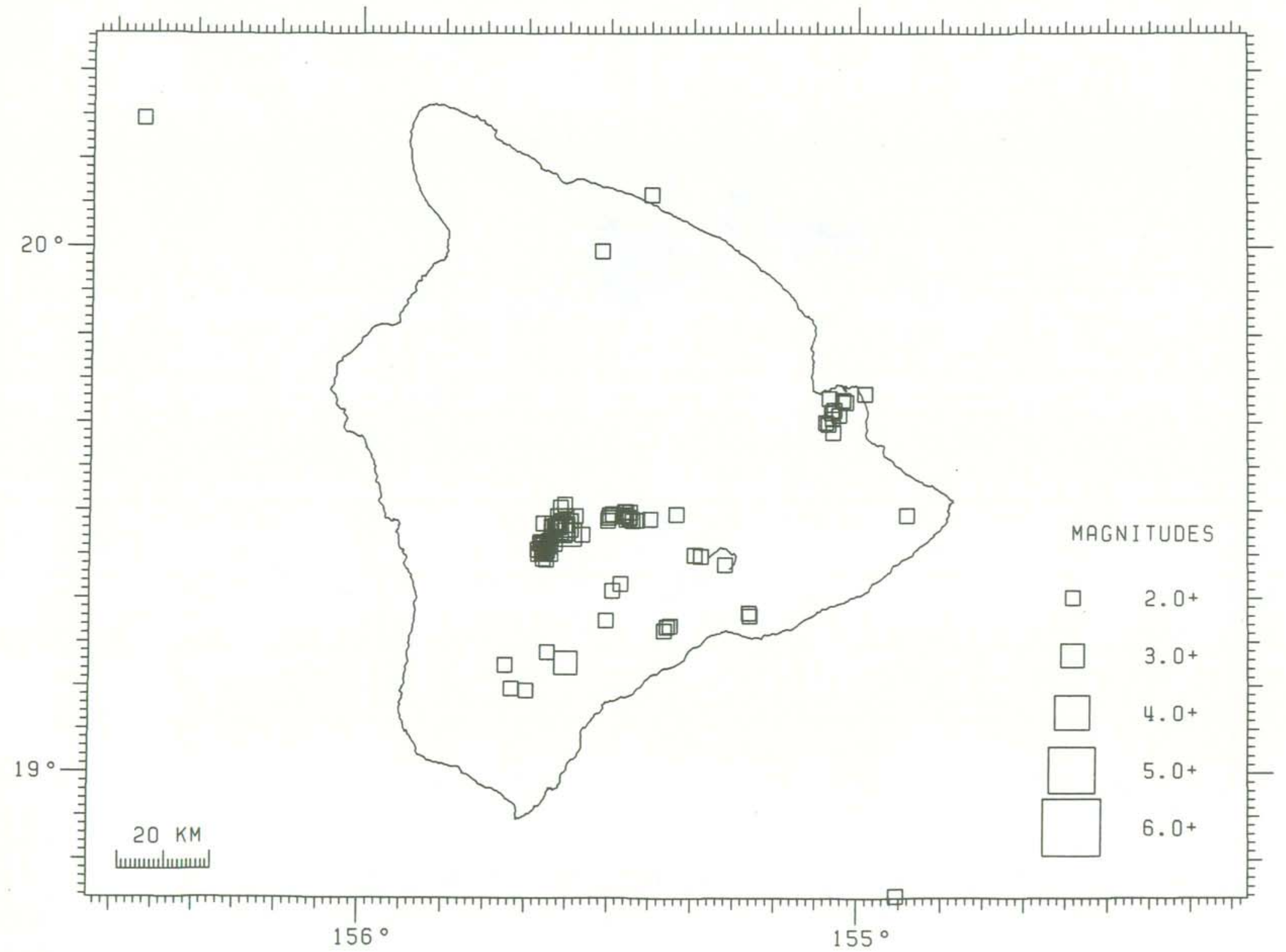


Figure 11

1984 HAWAII ISLAND EARTHQUAKE LOCATIONS

5. 1-13.0 KM DEPTHS, $M>=2$

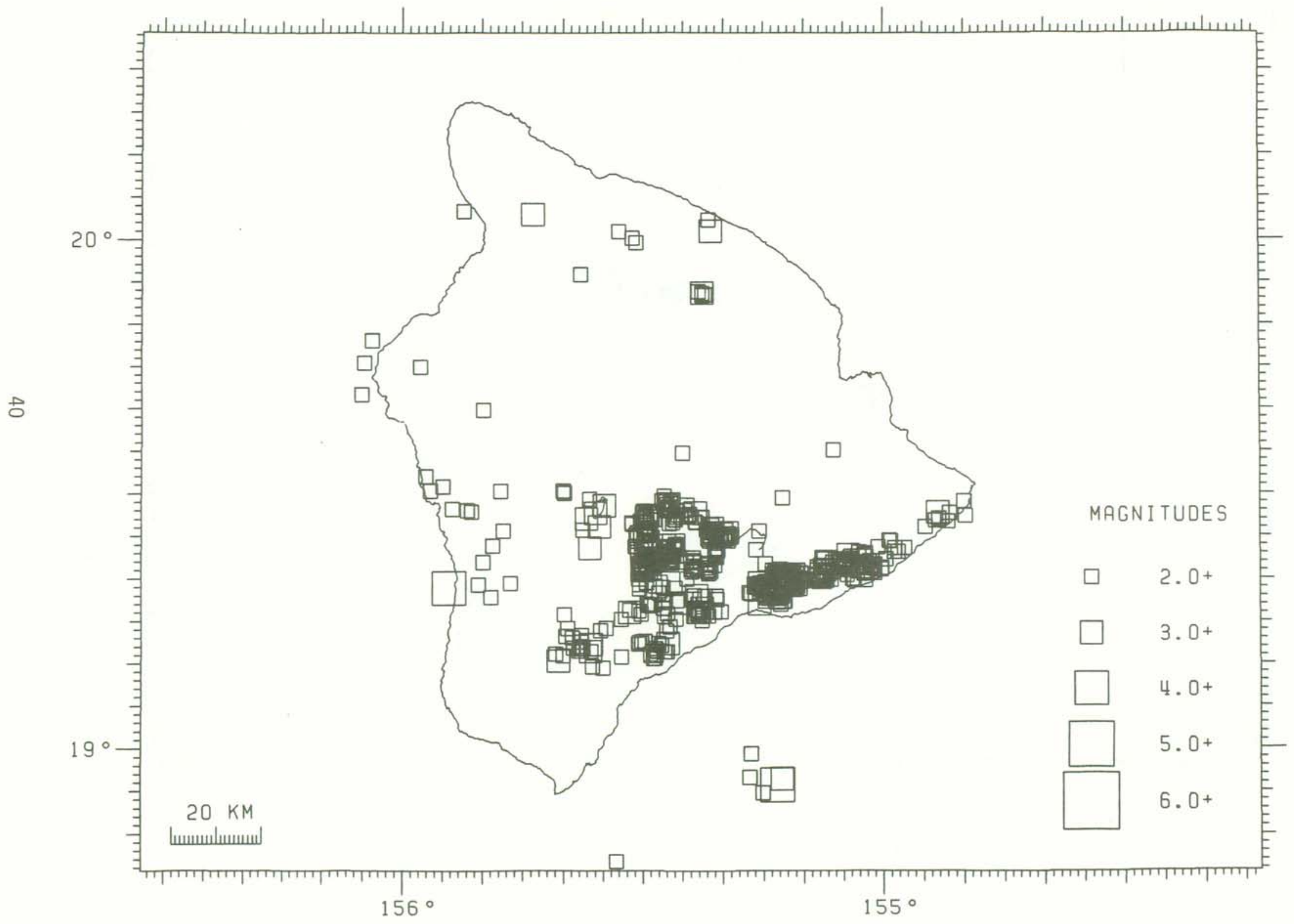


Figure 12

1984 HAWAII ISLAND EARTHQUAKE LOCATIONS

13.1-60.0 KM DEPTHS, $M>=2$

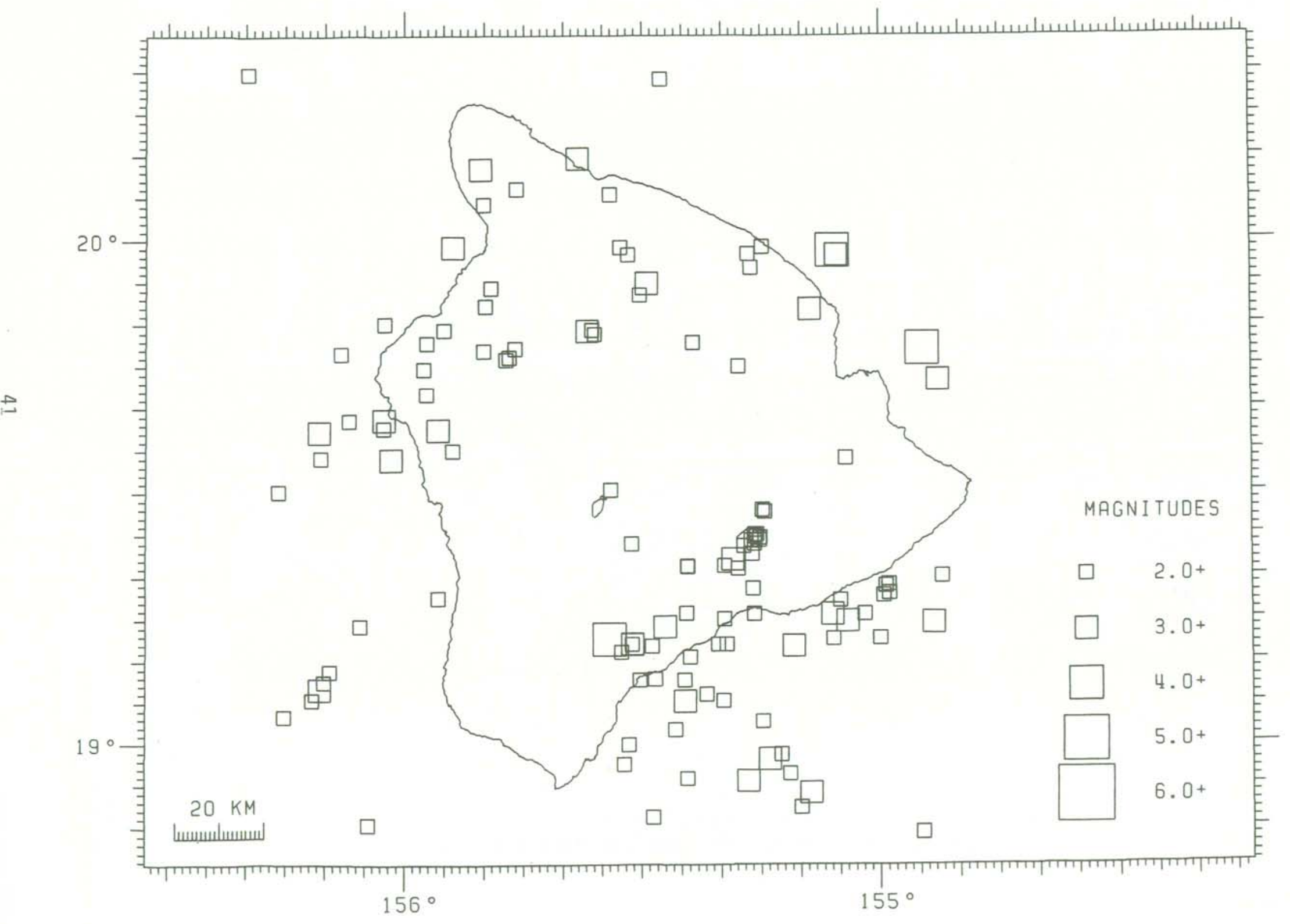


Figure 13

1984 KILAUEA SUMMIT EARTHQUAKE LOCATIONS

$0-5.0 \mathrm{KM}$ DEPTHS, $M>=1.0$

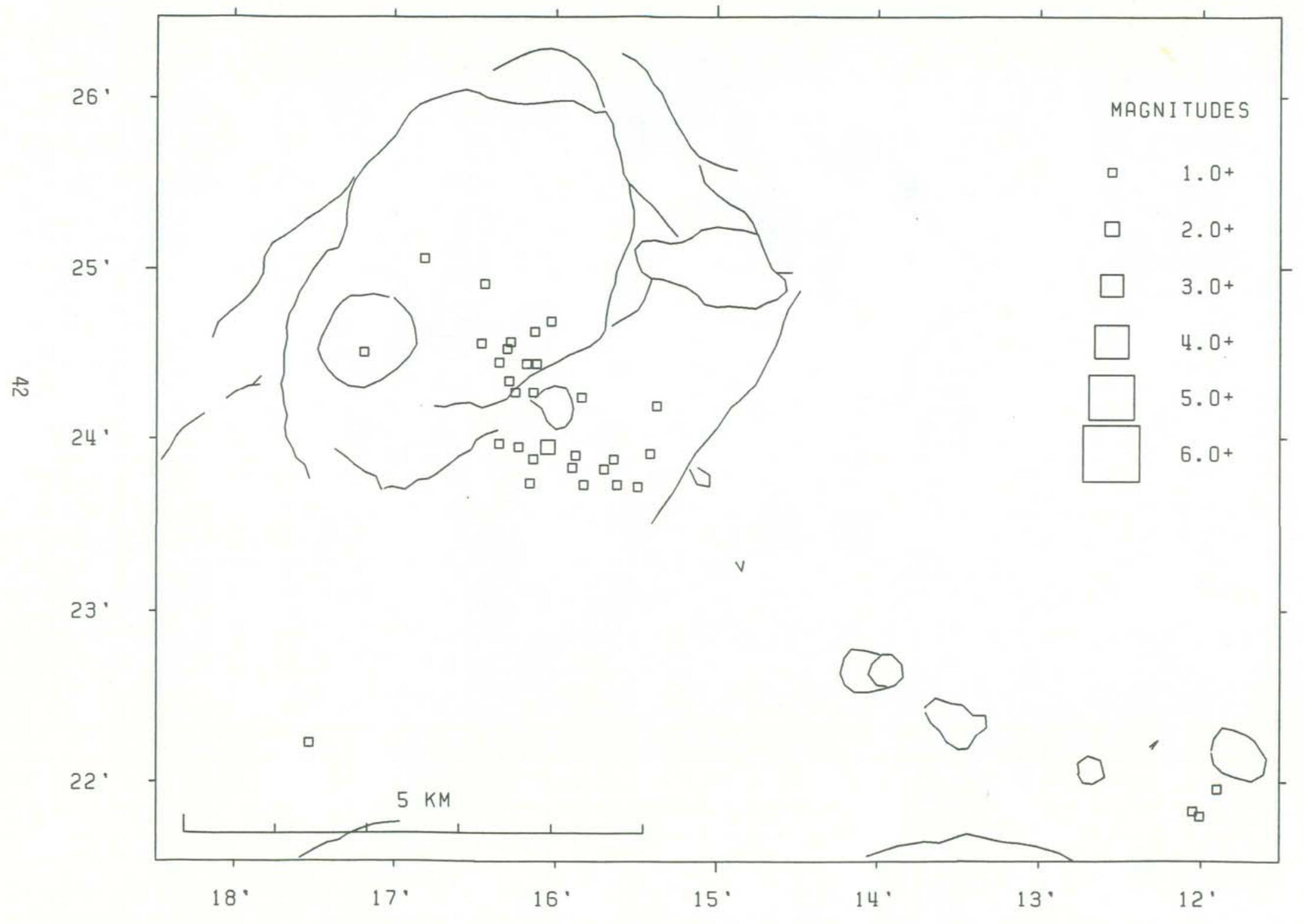


Figure 14

1984 KILAUEA SUMMIT EARTHQUAKE LOCATIONS

5. 1-13.0 KM DEPTHS, $M>=1.0$

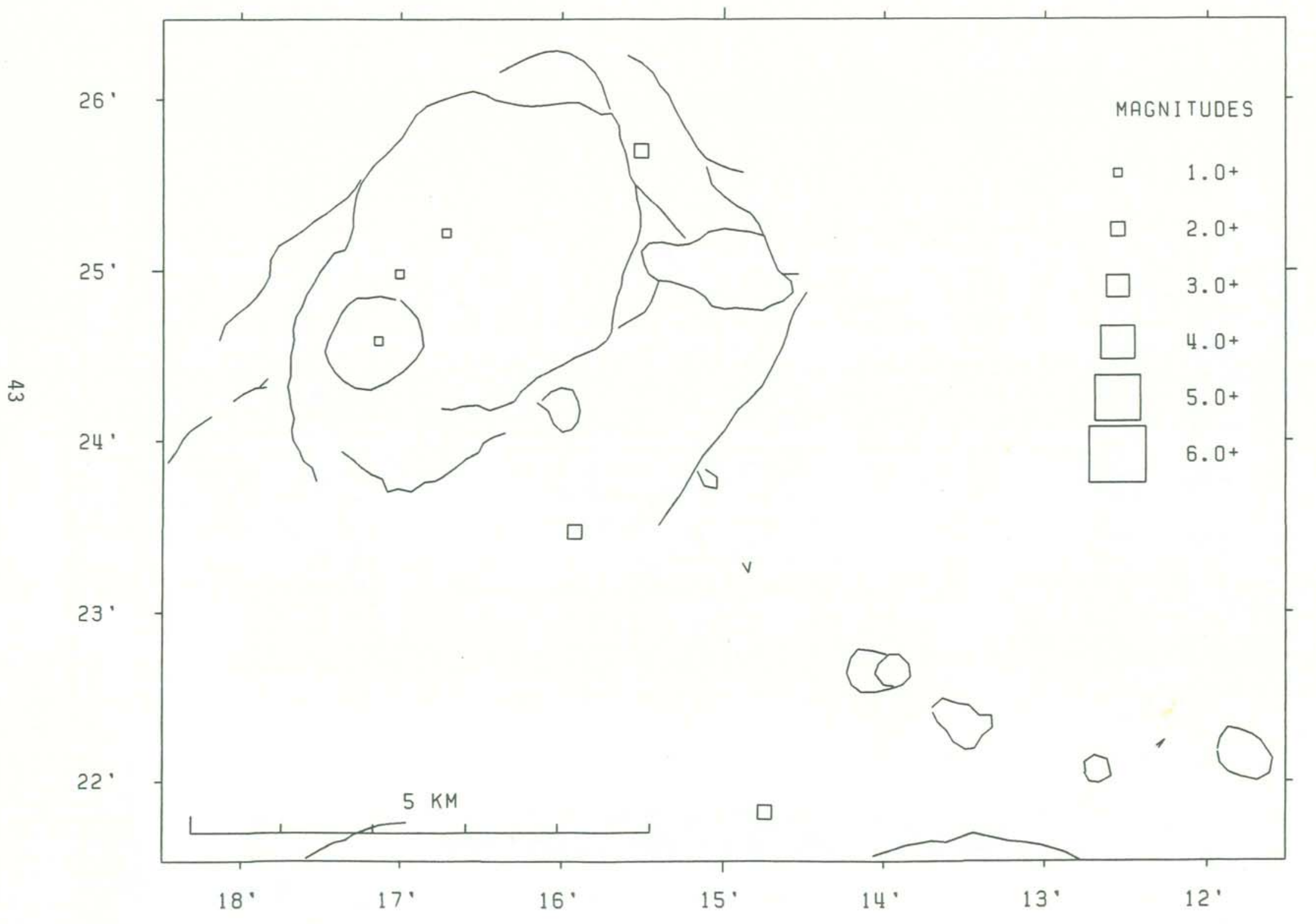


Figure 15

1984 KILAUEA SUMMIT EARTHQUAKE LOCATIONS

13. 1-60.0 KM DEPTHS, $M>=1.0$

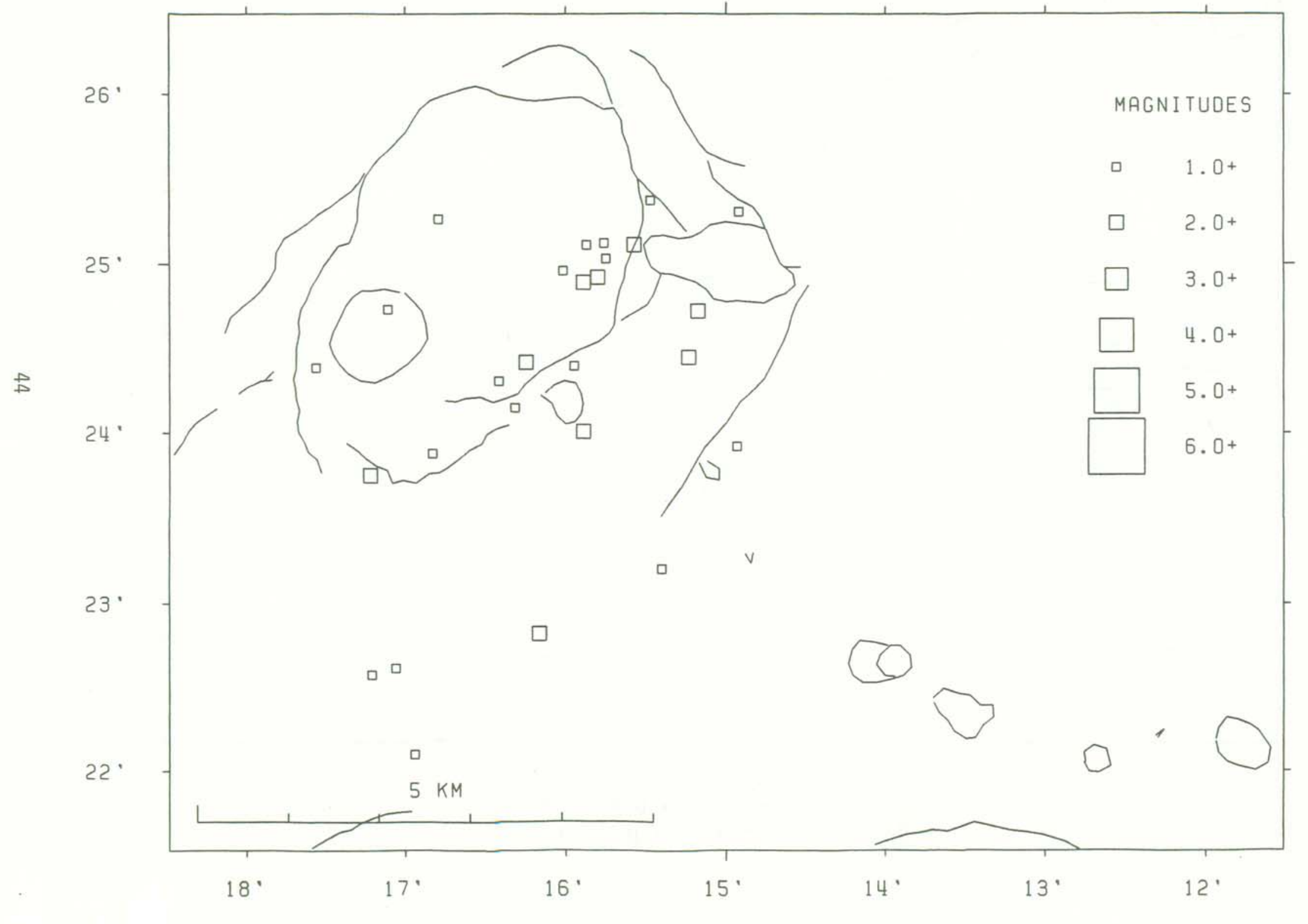


Figure 16

1984 KILAUEA SOUTH FLANK EARTHQUAKE LOCATIONS

$0-5.0 \mathrm{KM}$ DEPTHS, $M>=2.0$

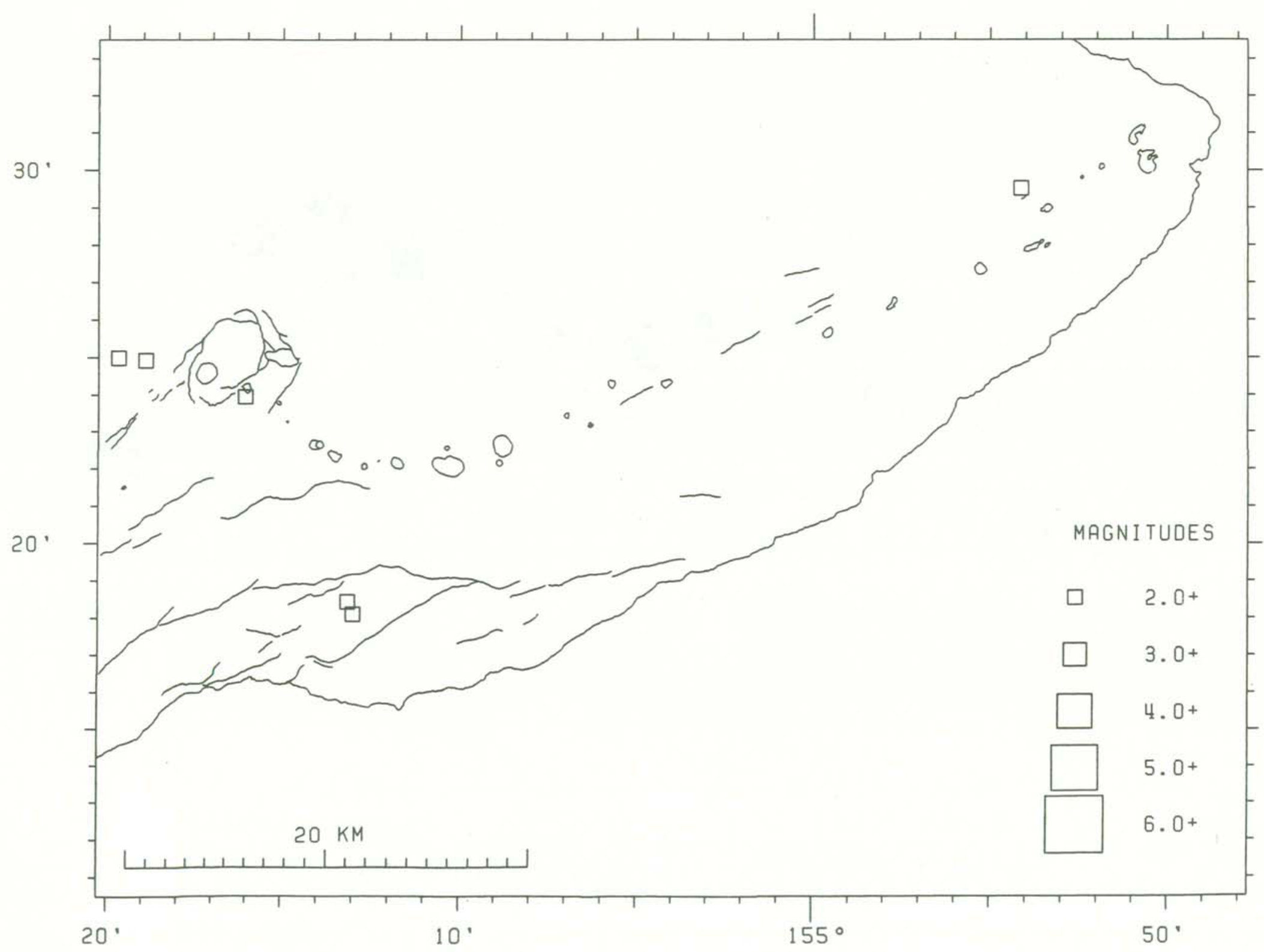


Figure 17

1984 KILAUEA SOUTH FLANK EARTHQUAKE LOCATIONS

5. $1-13.0$ KM DEPTHS, $M>=2.0$

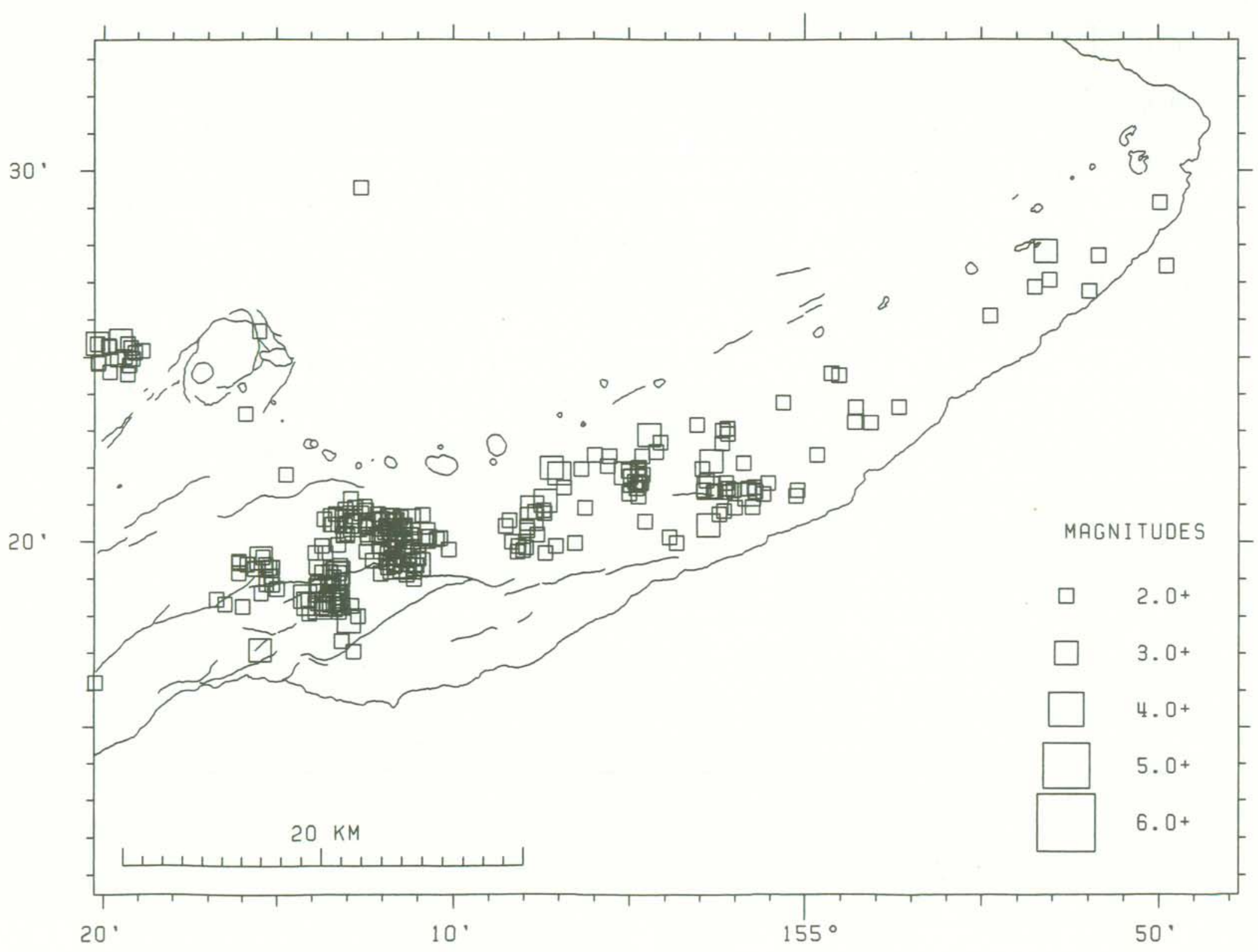


Figure 18

1984 KILAUEA SOUTH FLANK EARTHQUAKE LOCATIONS

13. 1-60.0 KM DEPTHS, $M>=2.0$

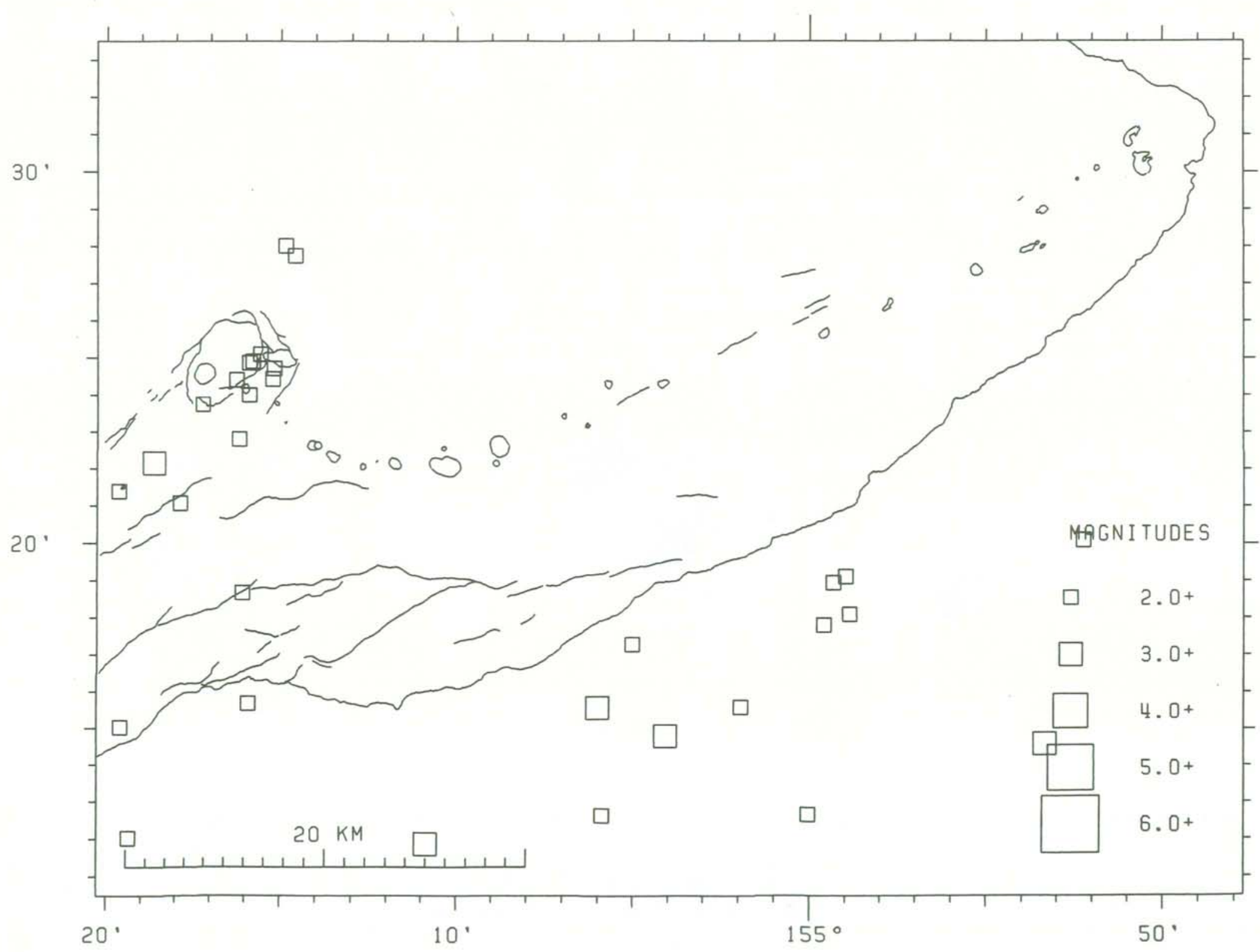


Figure 19

1984 MAUNA LOA SUMMIT EARTHQUAKE LOCATIONS

$0-5.0 \mathrm{KM}$ DEPTHS, $M>=2.0$

$\infty$

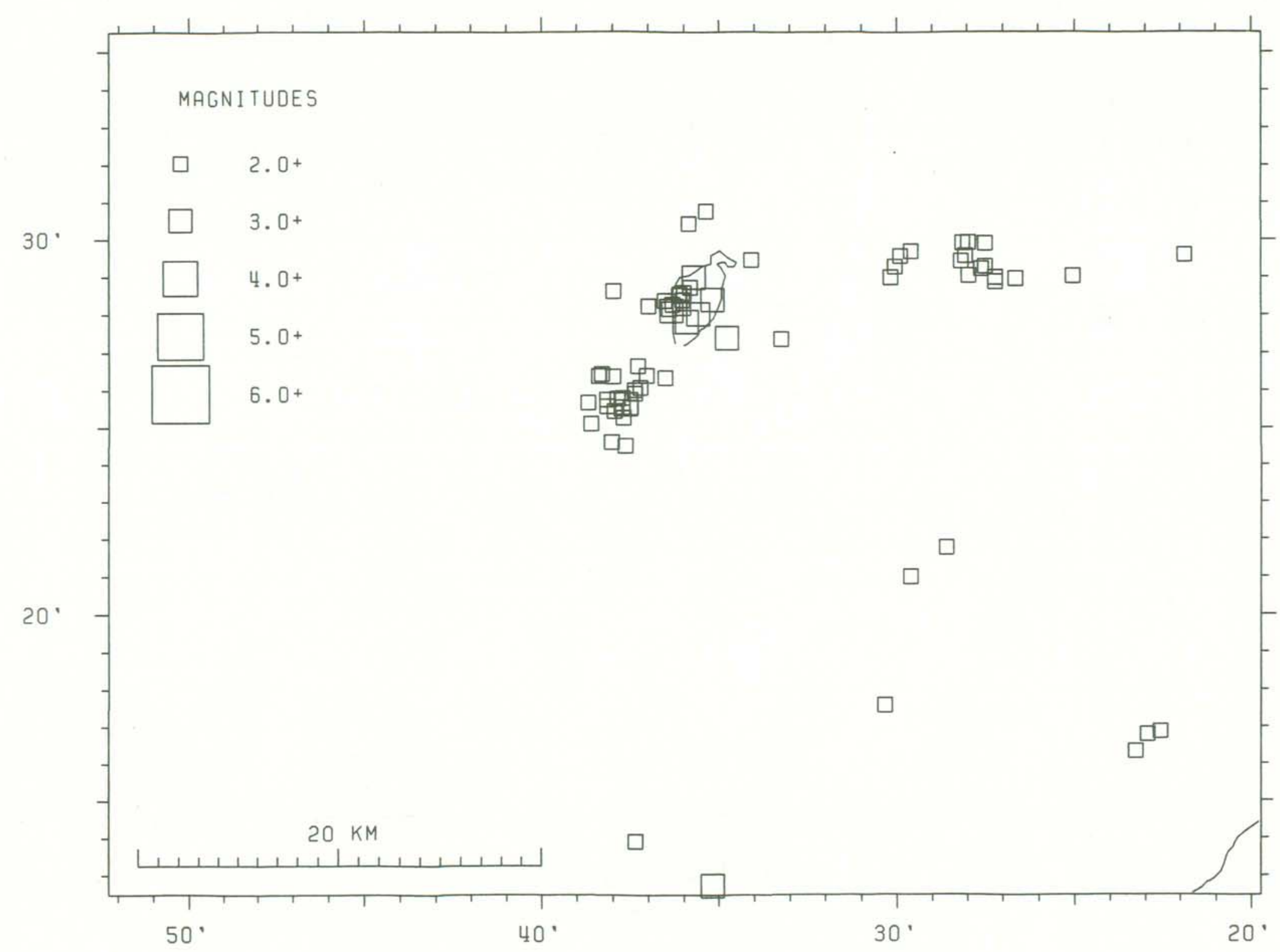


Figure 20

1984 MAUNA LOA SUMMIT EARTHQUAKE LOCATIONS

$5.1-13.0 \mathrm{KM}$ DEPTHS, $M>=2.0$

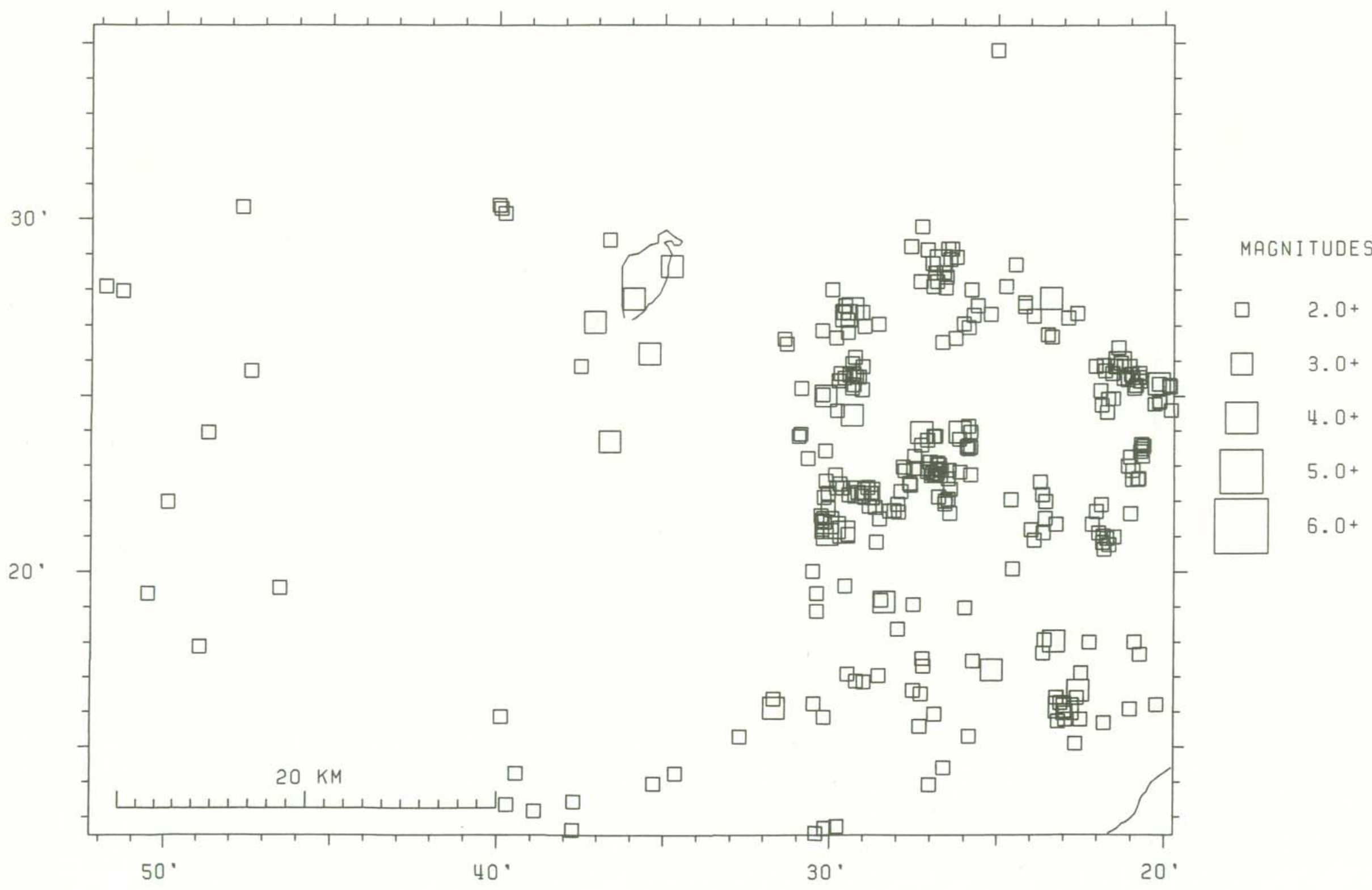


Figure 21

1984 MAUNA LOA SUMMIT EARTHQUAKE LOCATIONS

13. 1-60.0 KM DEPTHS, $M>=2.0$

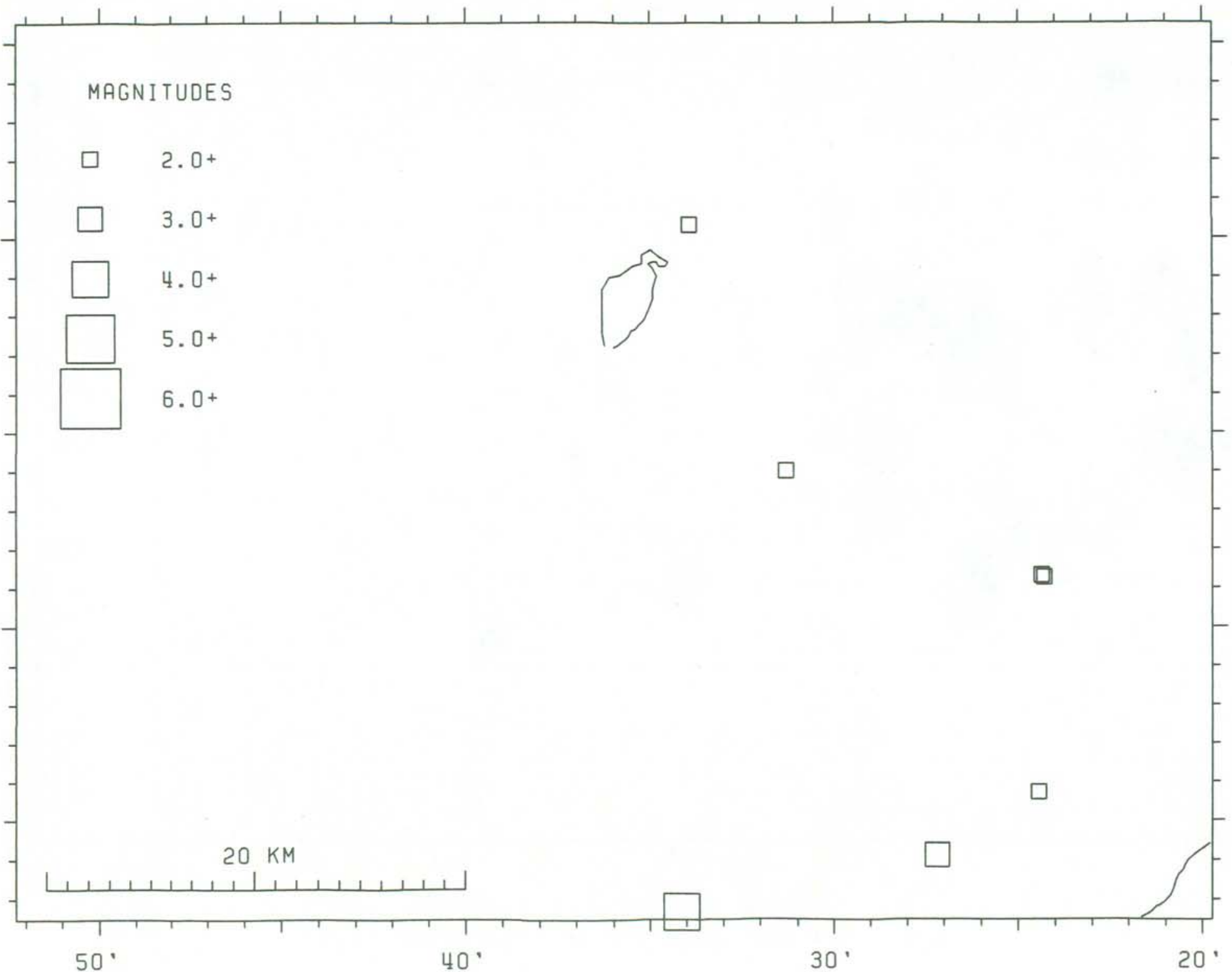


Figure 22

1984 HAWAII ISLAND EARTHQUAKE LOCATIONS

$0-60$ KM DEPTHS, $M>=3.0$

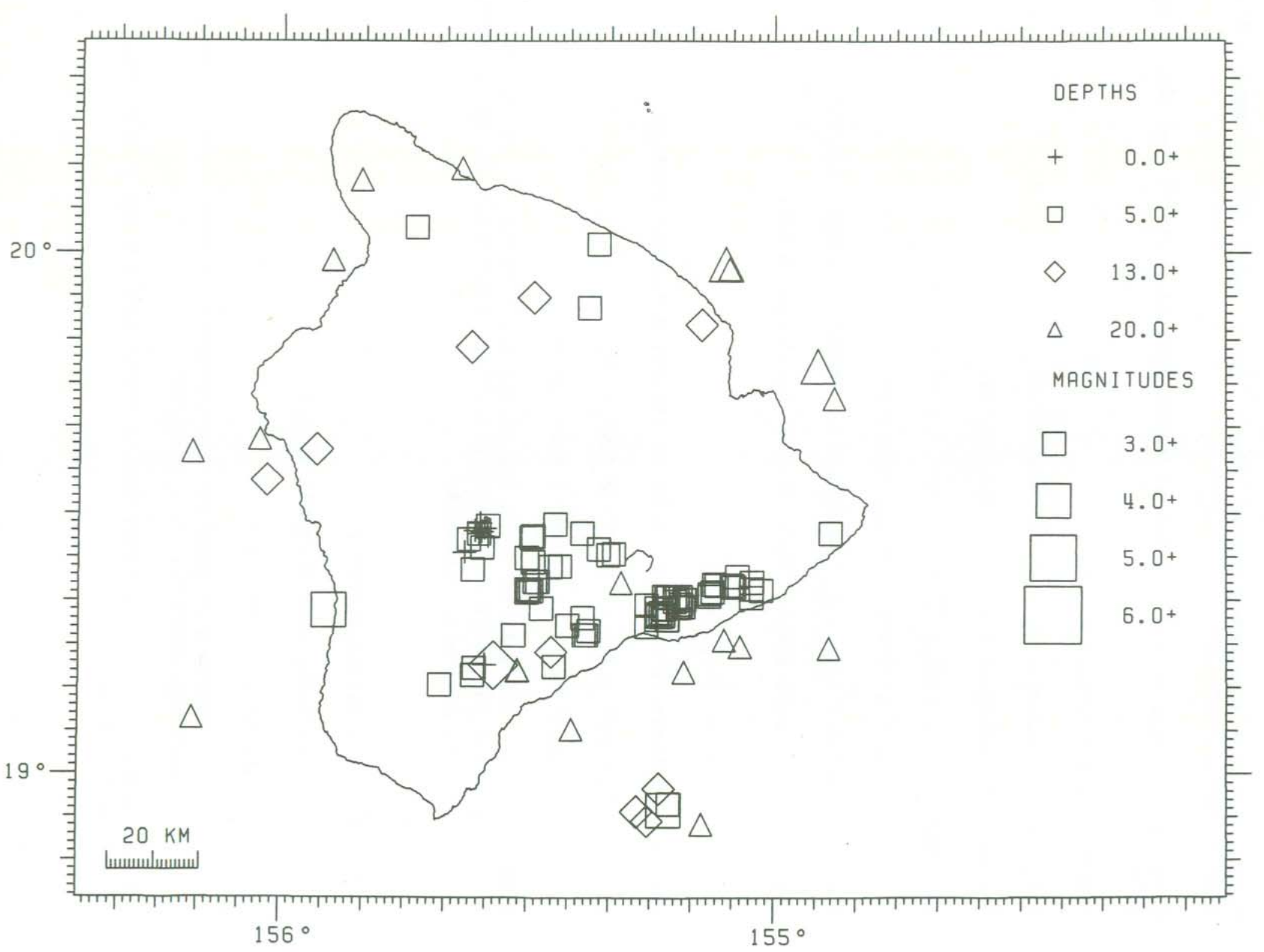


Table 5 is a chronological listing of successfully located earthquakes. For each event the following data are presented:

Origin time in Hawailan Standard Time: date, hour (HR), minute (MN), and second (SEC).

Epicenter in degrees and minutes of north latitude (LAT N) and west long1tude (LON W).

DEPTH - Depth of focus in $\mathrm{km}$.

AMP MAG - Amplitude magnitude, if determined.

DUR MAG - Duration magnitude, if determined.

NR - Number of arrivals ( $P$ or $S$ ) used for solution.

NS - Number of S arrivals used for solution.

GAP DEG - Largest azimuthal separation in degrees between stations.

RMS SEC - Root mean square error of time residuals in sec.

$$
\mathrm{RMS}=\left(\sum \mathrm{R}_{1}^{2} / \mathrm{NR}\right)^{1 / 2}
$$

MIN DIS - Epicentral distance in $\mathrm{km}$ to the third nearest station.

ERH $\mathrm{km}$ - Standard error of the epicenter in $\mathrm{km}$.

ERZ $\mathrm{km}$ - Standard error of depth of focus in $\mathrm{km}$.

REMK - Remarks, three letter code for geographic location of event. See Figures 5-8 for location of mnemonic code. Additional one letter codes have the following meanings:

$\mathrm{F}-\mathrm{felt}$

L - long period character

T - associated with harmonic tremor

B - quarry or other blast

* - the location program had a convergence problem, which usually means that the depth may be unreliable.

Table 5 lists all events located during 1984. Table 6 1ists only events of magnitude 3.0 or larger. 


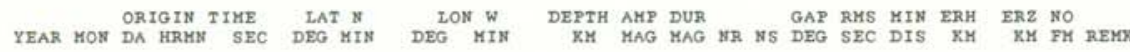

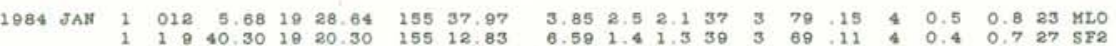

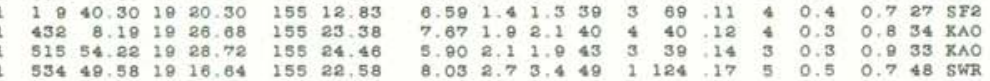

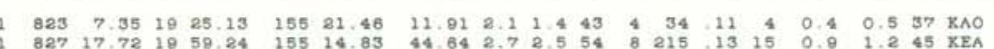

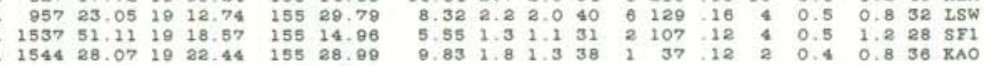

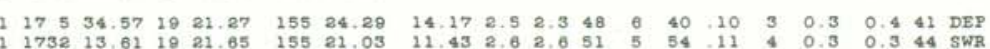

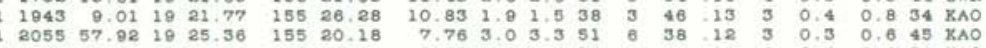

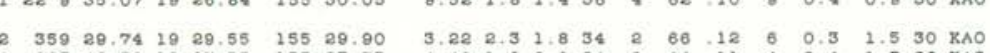

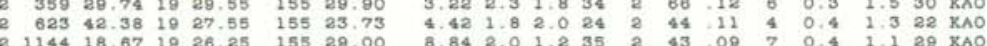

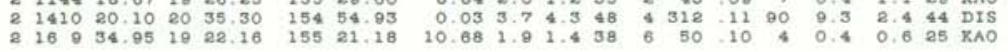

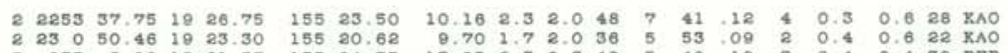

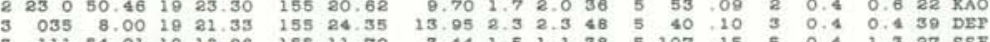

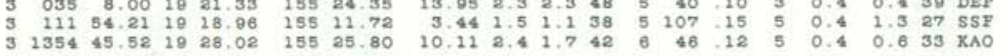

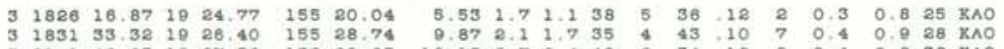

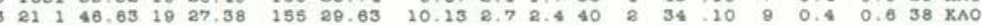

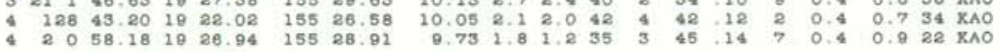

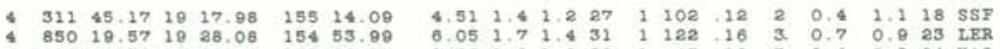

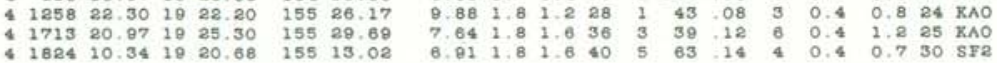

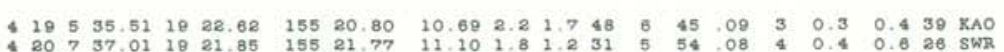

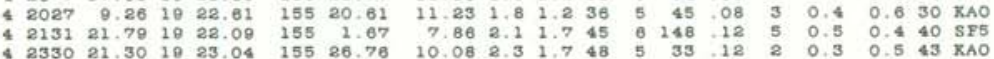

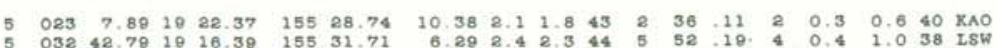

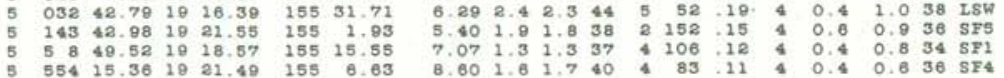

1984 HVO EARTHQUARE SUMMARY LIST PAGE 2

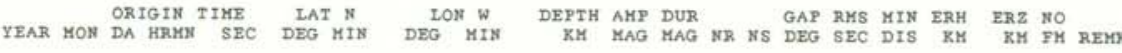

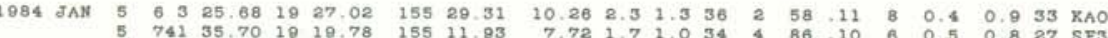

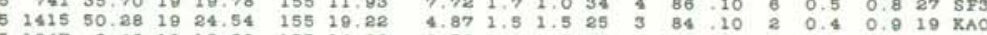

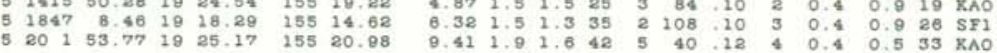

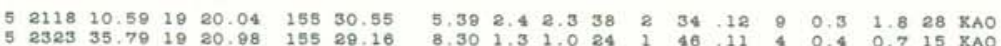

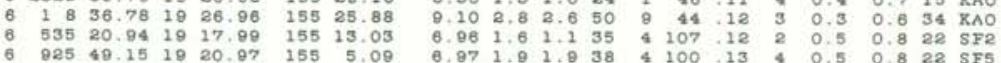

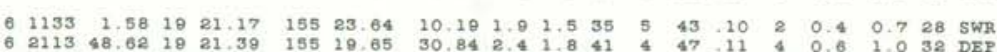

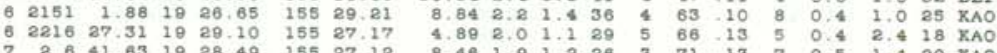

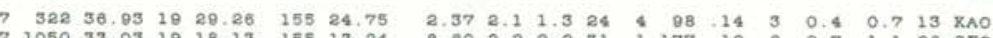

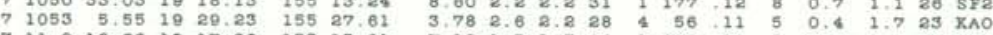

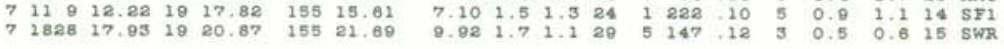

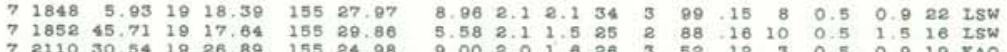

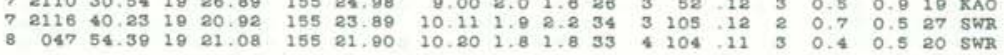
$\begin{array}{llllllllllllllllllll}8 & 1 & 3 & 41.87 & 19 & 27.39 & 155 & 29.06 & 10.46 & 2.4 & 2.1 & 31 & 3 & 60 & 11 & 8 & 0.4 & 0.6 & 25 & \text { KAO } \\ 8 & 115 & 1.94 & 18 & 22.88 & 155 & 27.77 & 10.28 & 2.4 & 2.5 & 31 & 3 & 68 & 10 & 1 & 0.4 & 0.5 & 27 & \text { KAO }\end{array}$

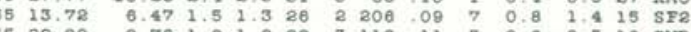

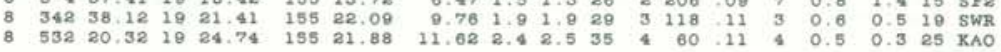

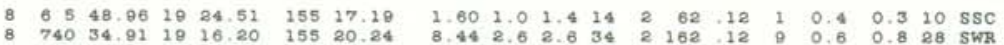

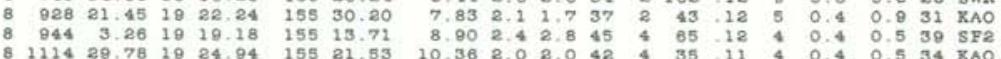

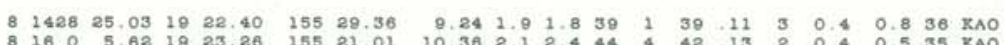

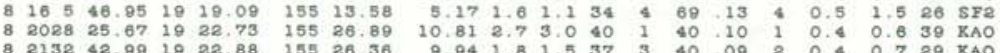

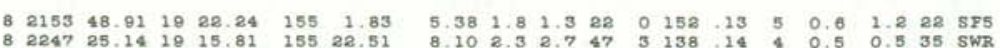

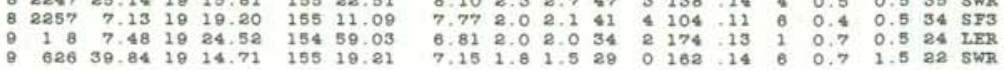




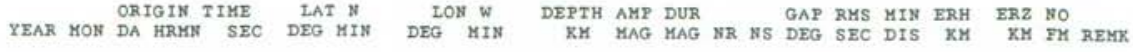

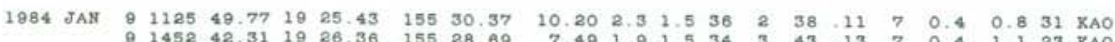

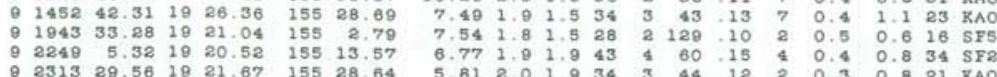

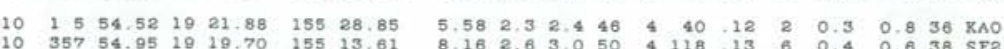

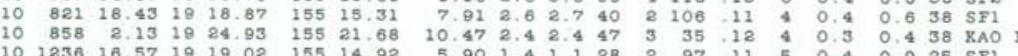

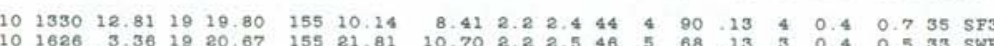

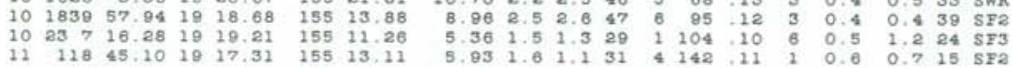

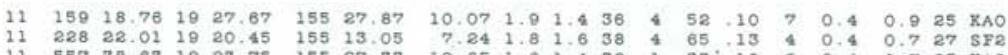

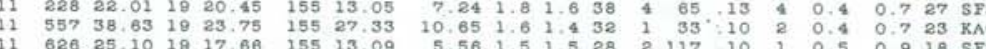

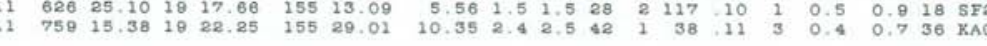

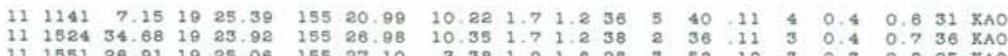

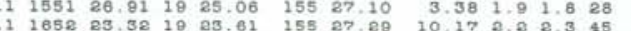

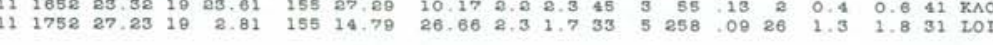

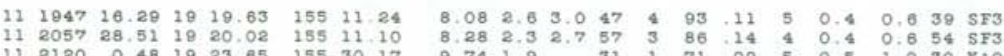

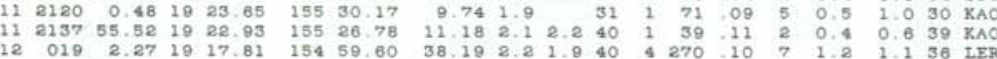

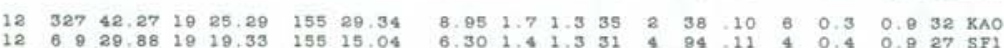

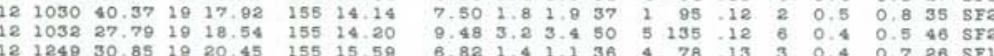

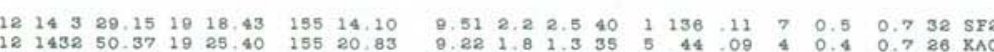

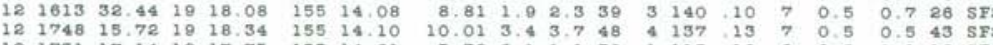

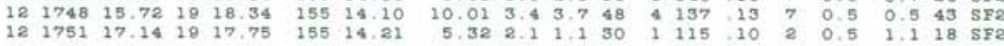

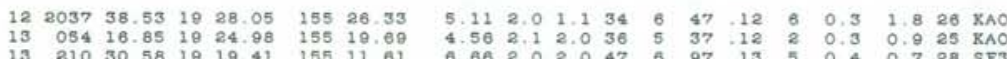

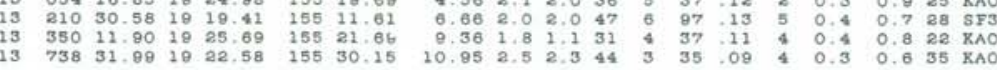

1984 HVO EARTHQUARE SUMMARY LIST

PAgE

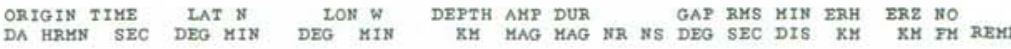

YEAR KON DN

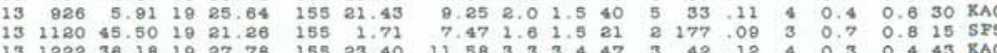

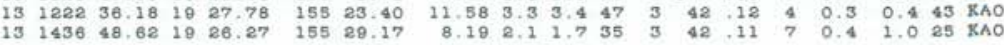

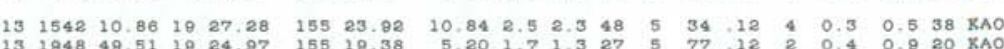

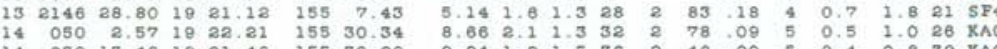

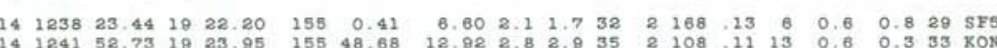

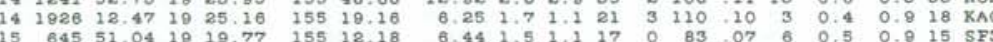

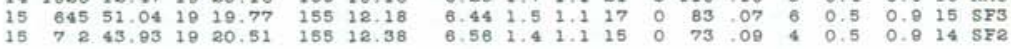
$\begin{array}{rrrrrrrrrrrrrrrrrrrrr}15 & 1256 & 3.05 & 19 & 19.36 & 155 & 12.23 & 6.31 & 1.6 & 1.6 & 26 & 2 & 91 & 08 & 5 & 0.4 & 1.0 & 24 & \text { SF3 } \\ 15 & 1655 & 38 & 06 & 10 & 21.67 & 155 & 0.52 & 8.04 & 1.8 & 1.4 & 31 & 1 & 177 & 11 & 5 & 0.8 & 0.7 & 28 & \text { SF9 }\end{array}$

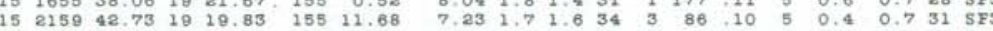

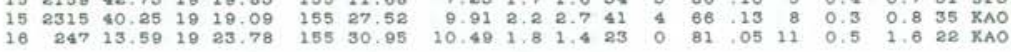

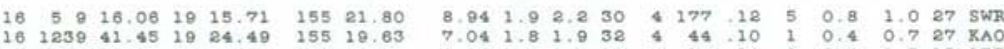

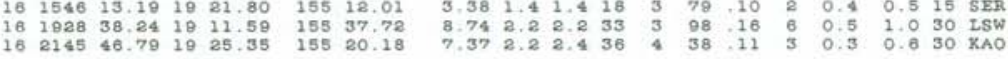

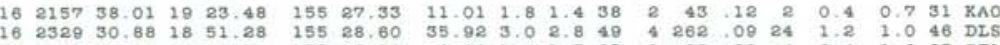

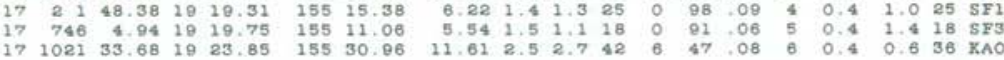

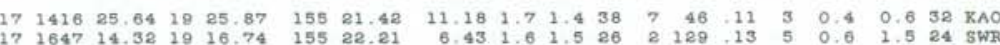

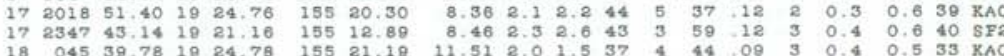

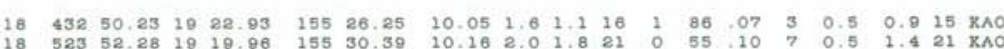

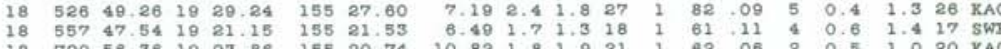

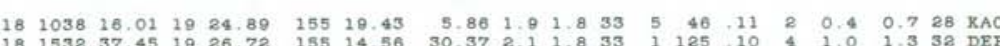

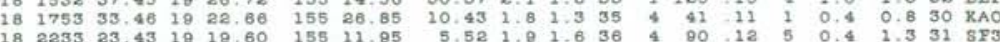

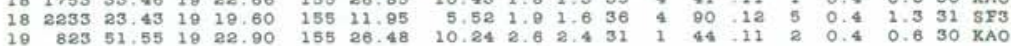


1984 HVO EARTHQUAKE SUMMARY LIST

PAGE 5

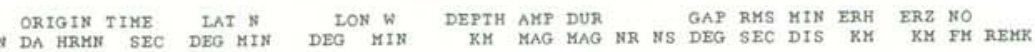

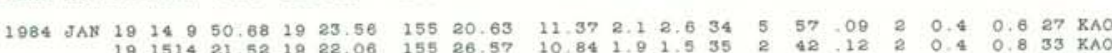

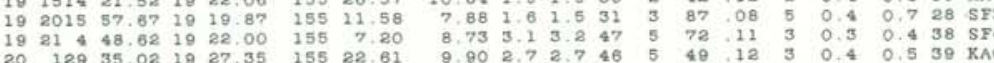

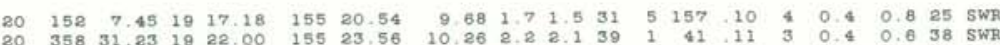

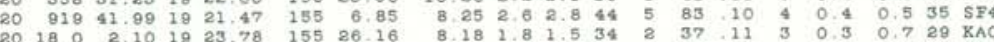

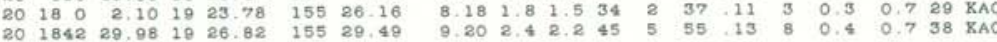

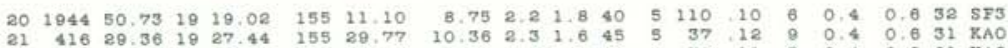

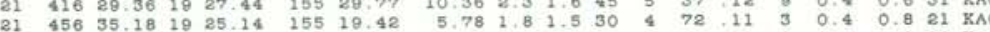

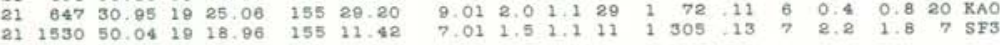

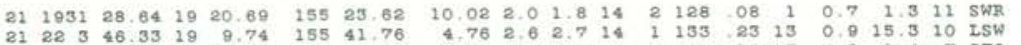

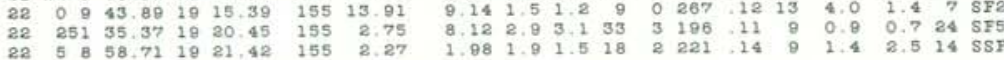

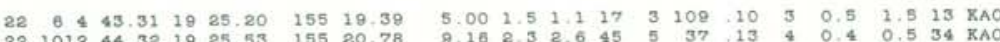

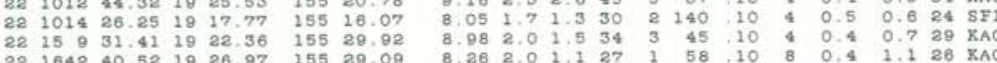

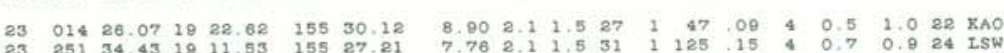

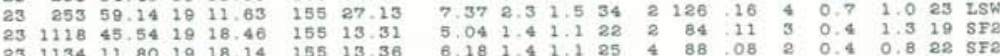

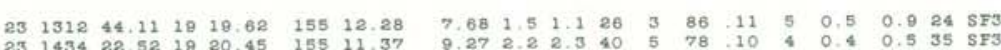

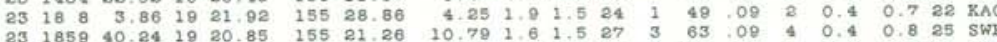

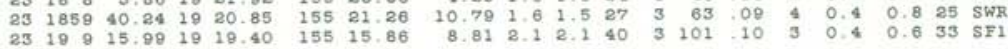
$\begin{array}{rrrrrrrrrrrrrrrrrrr}23 & 2121 & 29.20 & 19 & 18.68 & 155 & 12.97 & 6.74 & 1.8 & 1.8 & 39 & 3 & 91 & 13 & 3 & 0.4 & 0.9 & 33 & \text { SF2 } \\ 23 & 2150 & 35.10 & 18 & 25.51 & 155 & 21.09 & 10.05 & 2.0 & 1.8 & 42 & 6 & 37 & 12 & 4 & 0.3 & 0.6 & 35 & \text { KAO }\end{array}$

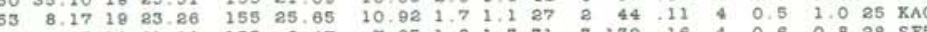

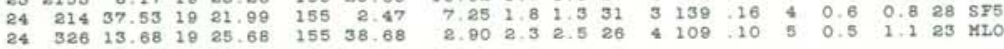

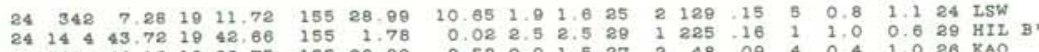

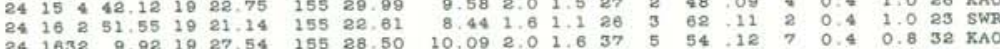

1984 HVO EARTHQUAKE SUMMARY LIST

PAGE 6 ORIGIR TIME
DA HRT N

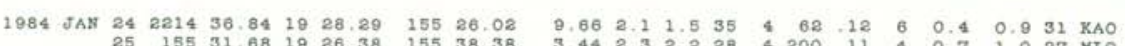

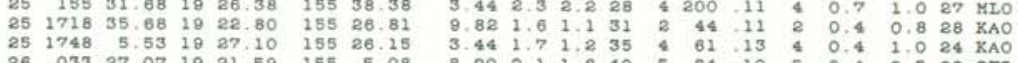

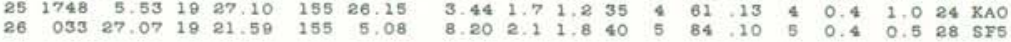

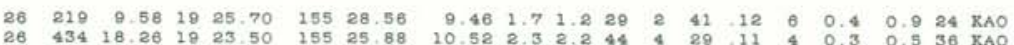

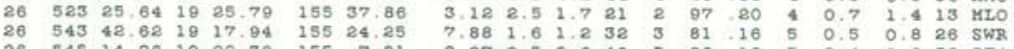

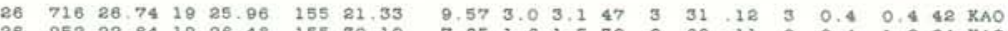

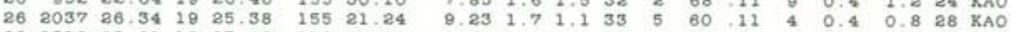

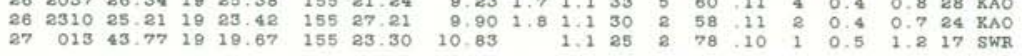
$\begin{array}{rrrrrrrrrrrrrrrrrrr}27 & 443 & 39.44 & 19 & 11.58 & 155 & 27.22 & 8.15 & 1.8 & 1.2 & 21 & 1 & 147 & 10 & 4 & 0.7 & 0.7 & 10 & \text { ISW } \\ 27 & 659 & 18.02 & 19 & 27.58 & 155 & 28.57 & 10.58 & 2.3 & 1.9 & 43 & 3 & 53 & 11 & 8 & 0.3 & 0.6 & 31 & \text { KAO }\end{array}$

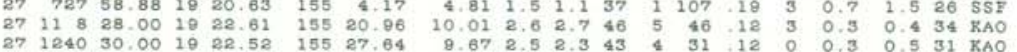

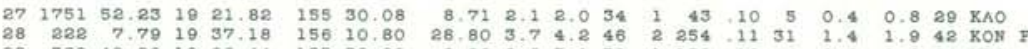

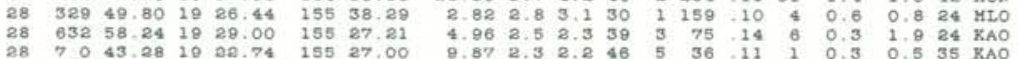

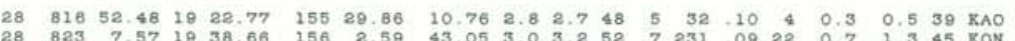

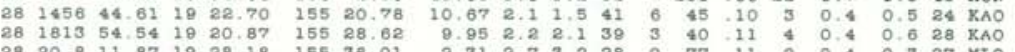
$\begin{array}{llllllllllllllllll}28 & 2143 & 22.58 & 19 & 24.02 & 155 & 31.30 & 14.33 & 2.2 & 2.3 & 29 & 1 & 81 & 08 & 3 & 0.5 & 0.6 & 22 \\ 2 M L\end{array}$

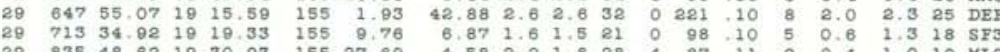

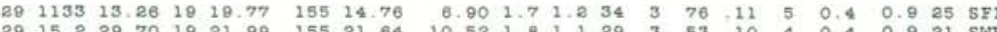

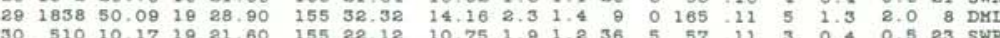

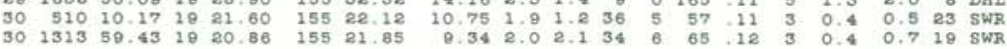
$\begin{array}{llllllllllllllllllllll}30 & 14 & 1 & 8.46 & 10 & 20.64 & 155 & 21.76 & 0.35 & 1.9 & 1.5 & 32 & 4 & 68 & 12 & 3 & 0.4 & 0.8 & 25 & \text { SWR } \\ 30 & 1931 & 1.81 & 19 & 29.67 & 155 & 29.60 & 2.71 & 2.5 & 1.5 & 43 & 5 & 89 & 12 & 0 & 0.3 & 1.1 & 31 & \text { KAO }\end{array}$

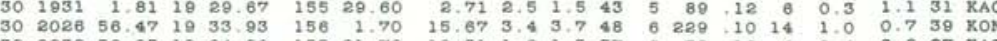

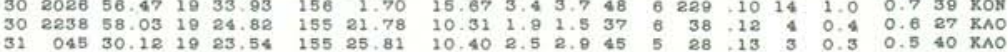




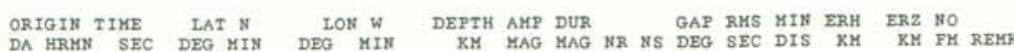

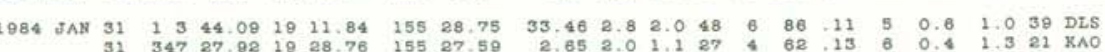

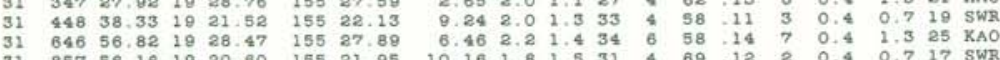

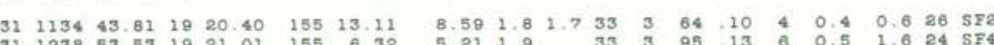

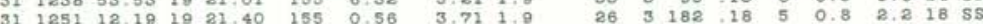

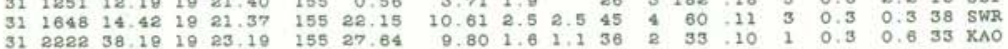

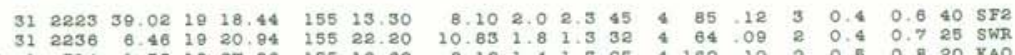

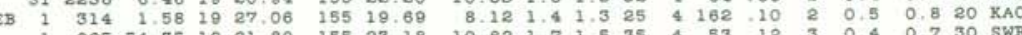

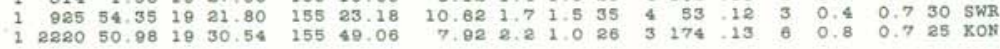

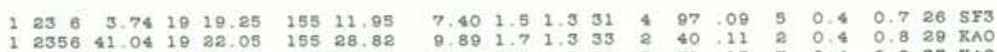

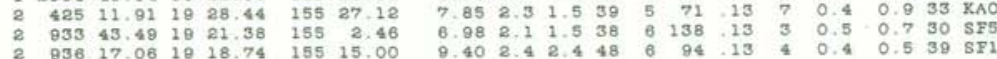

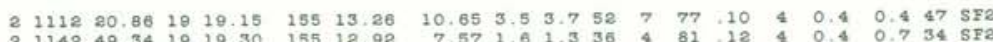

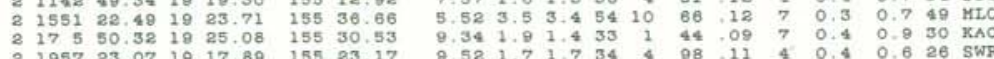

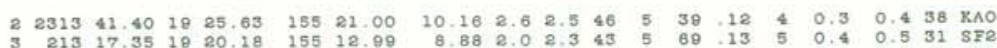

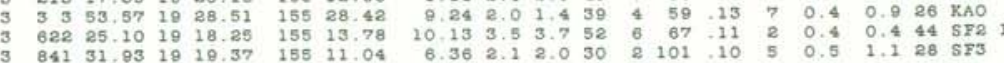

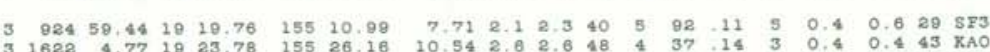

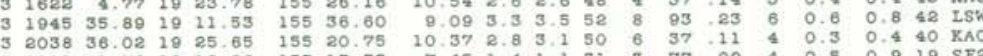

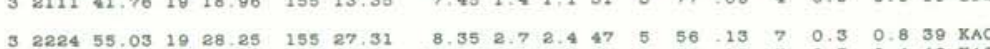

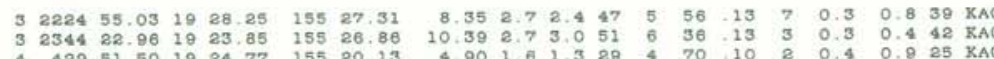

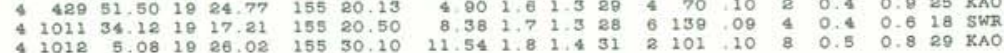

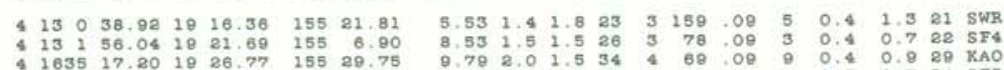

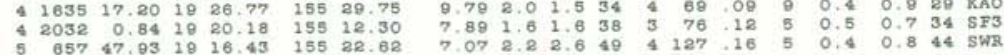

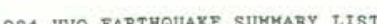

PAGE \&

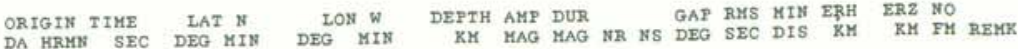

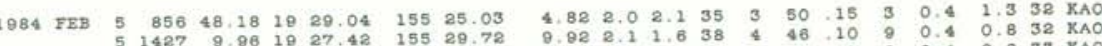

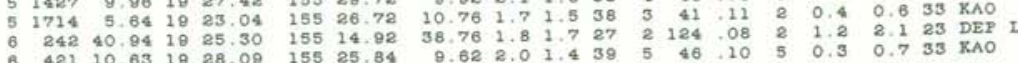

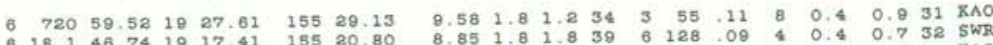

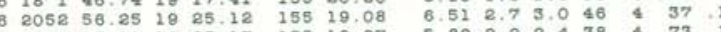

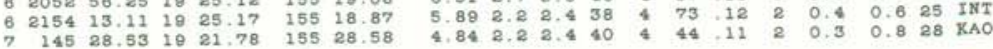
$\begin{array}{lllllllllllllllllll}7 & 340 & 59.84 & 19 & 24.14 & 155 & 25.88 & 10.46 & 2.5 & 2.5 & 49 & 5 & 28 & 13 & 2 & 0.3 & 0.4 & 41 & \text { KAO } \\ 7 & 342 & 33.54 & 19 & 22.51 & 155 & 20.80 & 9.87 & 1.9 & 1.5 & 38 & 7 & 40 & 12 & 3 & 0.4 & 0.6 & 27 & \text { KAO }\end{array}$

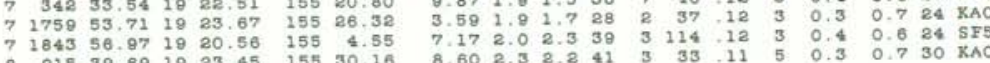

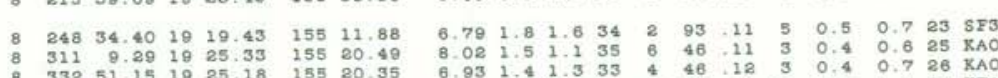

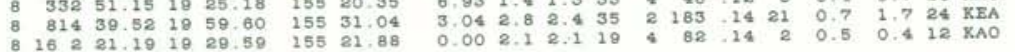

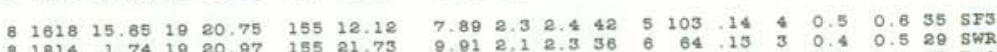

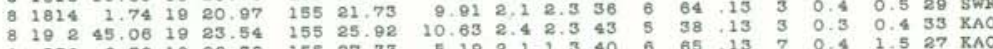

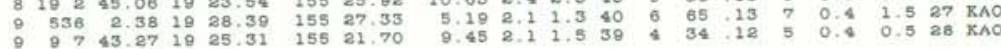

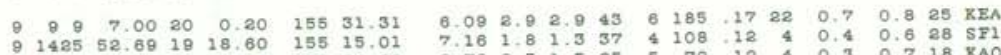

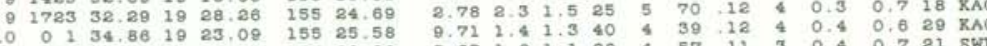

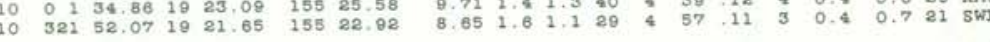
$\begin{array}{rrrrrrrrrrrrrrrrrrr}10 & 954 & 5.48 & 19 & 23.91 & 155 & 26.97 & 9.80 & 1.7 & 1.2 & 34 & 2 & 48 & 11 & 3 & 0.4 & 0.7 & 27 & \text { KAO } \\ 10 & 1935 & 13.47 & 19 & 26.85 & 155 & 23.59 & 11.19 & 1.8 & 1.2 & 24 & 3 & 69 & 00 & 4 & 0.5 & 1.0 & 10 & \text { KAO }\end{array}$

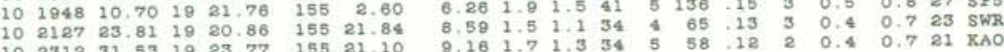

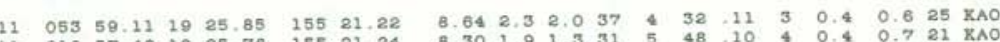

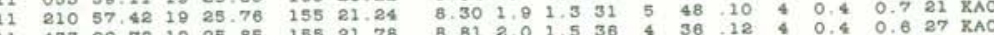

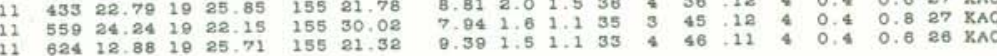

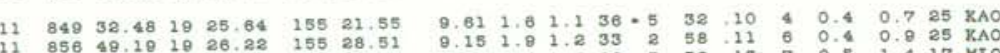

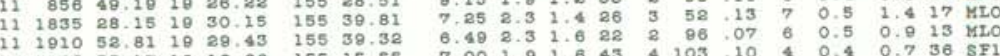




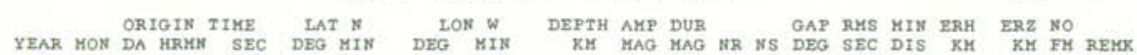

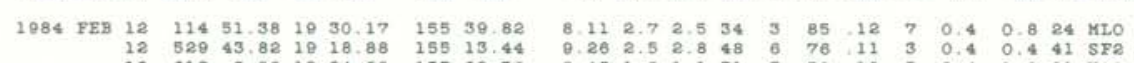

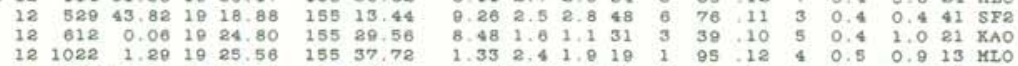

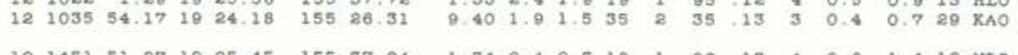

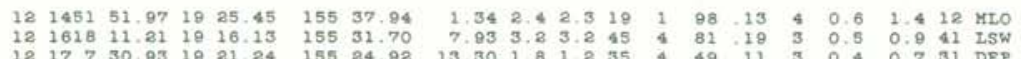

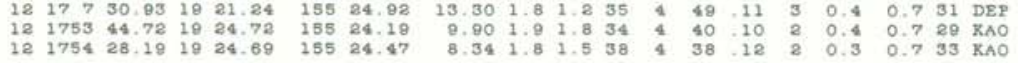

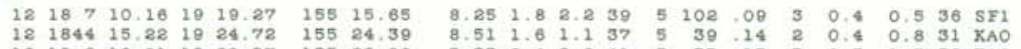

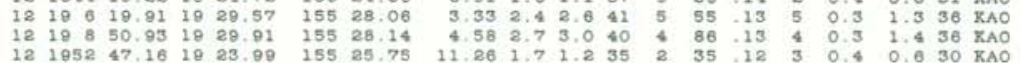

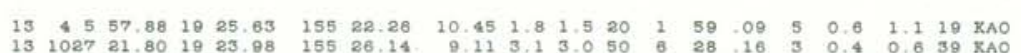

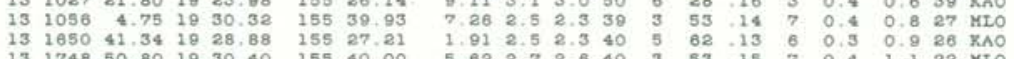

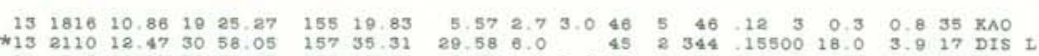

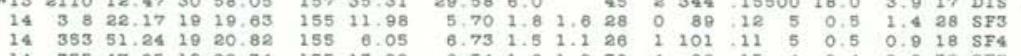

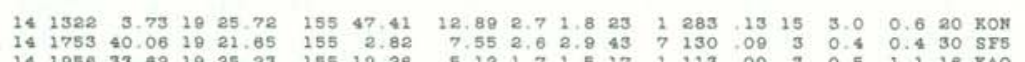

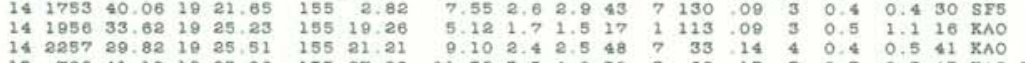
$\begin{array}{lllllllllllllllllll}15 & 1124 & 47.34 & 19 & 19.47 & 155 & 29.64 & 9.33 & 1.5 & 1.2 & 18 & 2 & 52 & 09 & 7 & 0.4 & 1.1 & 17 & \text { KAO } \\ 15 & 16 & 43.71 & 10 & 23.88 & 155 & 16.14 & 2.69 & 1.4 & 1.2 & 15 & 2 & 103 & 10 & 1 & 0.3 & 0.3 & 11 & \text { SEC }\end{array}$

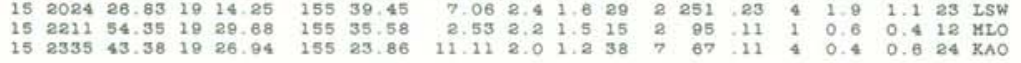
$\begin{array}{llllllllllllllllllll}16 & 652 & 33.13 & 19 & 28.85 & 155 & 26.72 & 11.97 & 3.2 & 3.1 & 54 & 7 & 41 & 13 & 6 & 0.3 & 0.4 & 46 & \text { KAO } \\ 16 & 741 & 52.67 & 19 & 19.00 & 155 & 15.16 & 7.08 & 1.4 & 1.3 & 33 & 2 & 101 & 00 & 4 & 0.4 & 0.8 & 30 & \text { SF1 }\end{array}$

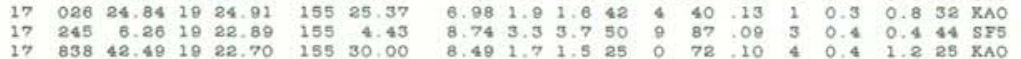

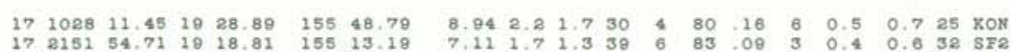

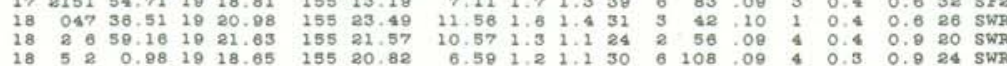

*NEIS determination of 4.8 magnitude based on World Wide Seismograph Network Stations.

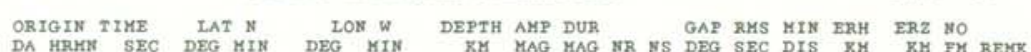

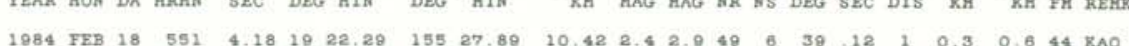

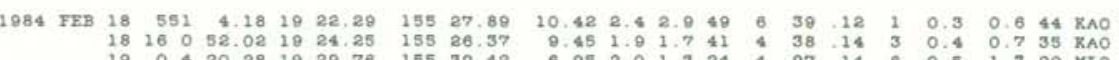

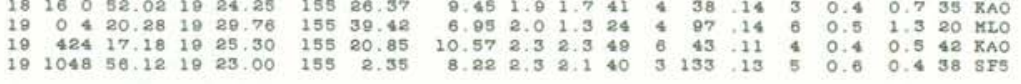

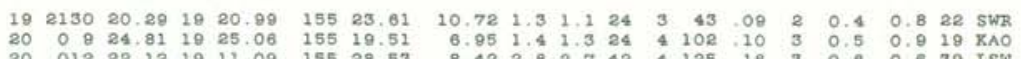

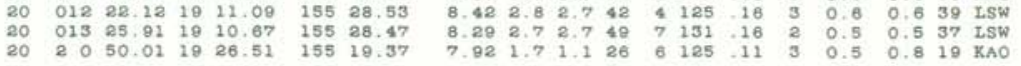
$\begin{array}{lllllllllllllllllll}20 & 344 & 29.55 & 19 & 22.09 & 155 & 27.20 & 10.86 & 1.6 & 1.4 & 37 & 3 & 42 & 11 & 1 & 0.4 & 0.6 & 30 & \text { KAO } \\ 20 & 540 & 31.87 & 19 & 27.00 & 155 & 29.24 & 10.54 & 2.2 & 1.4 & 39 & 5 & 46 & 11 & 8 & 0.3 & 0.7 & 20 & \text { KAO }\end{array}$

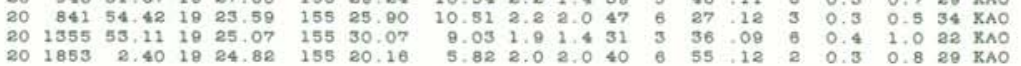

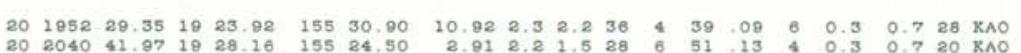

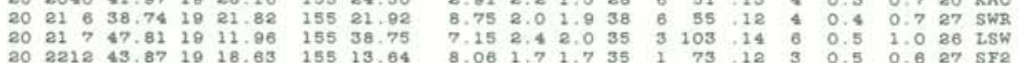

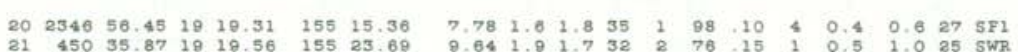

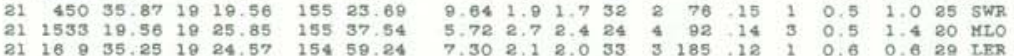

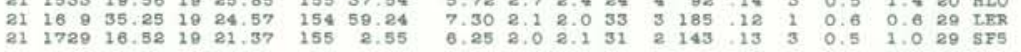

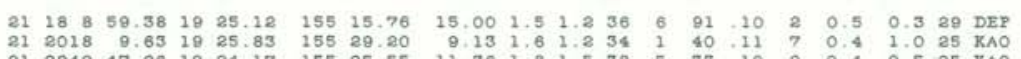

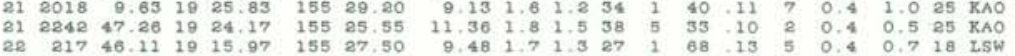

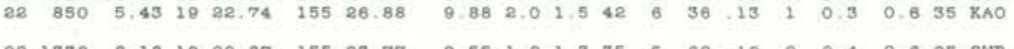

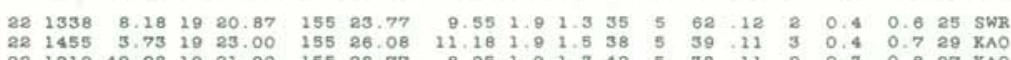

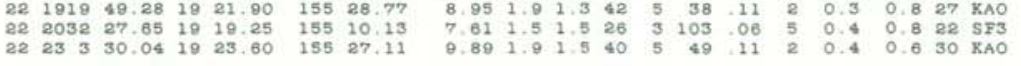

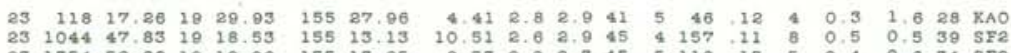

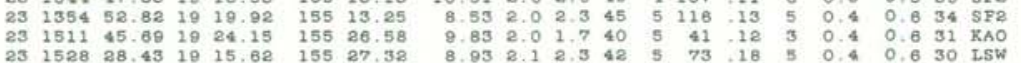

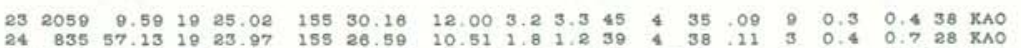

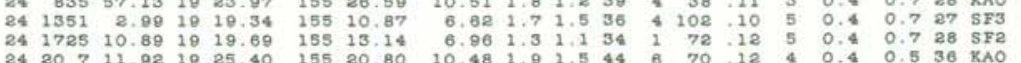




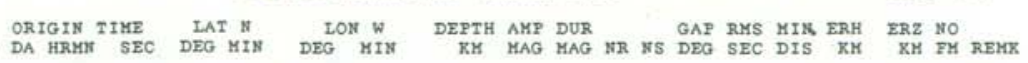

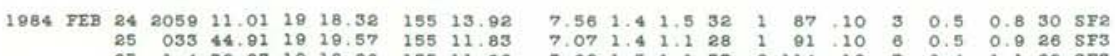

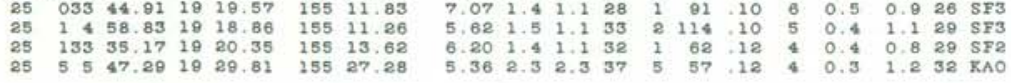

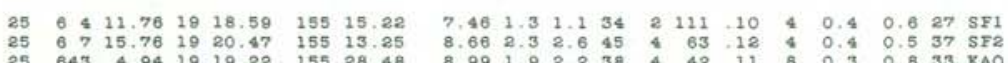

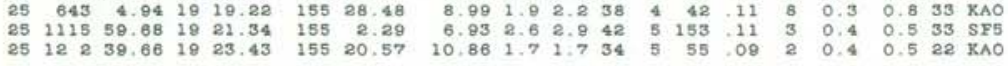
$\begin{array}{llllllllllllllllllll}25 & 1844 & 19.09 & 19 & 25.21 & 155 & 19.11 & 6.98 & 1.9 & 1.5 & 28 & 5 & 75 & 13 & 3 & 0.4 & 0.8 & 19 & \text { KAO } \\ 25 & 190 & 22.65 & 19 & 17.62 & 155 & 12.98 & 5.85 & 1.6 & 1.6 & 32 & 1 & 125 & 10 & 1 & 0.6 & 1.0 & 23 & \text { SF2 }\end{array}$

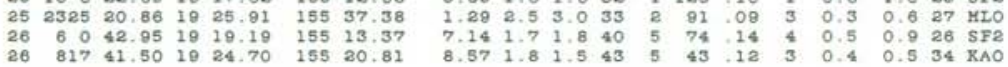

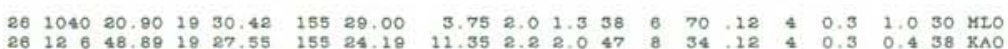

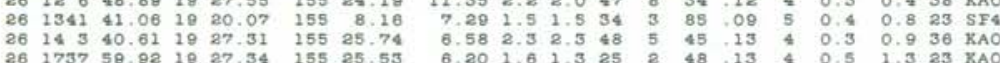
$\begin{array}{llllllllllllllllllll}26 & 2012 & 25.58 & 19 & 15.47 & 155 & 15.28 & 6.33 & 1.5 & 1.5 & 30 & 2 & 189 & 10 & 4 & 0.7 & 1.0 & 28 & 5 F 1\end{array}$

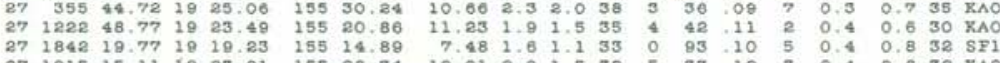

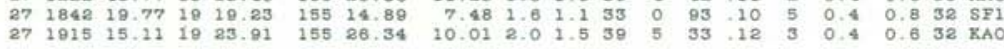

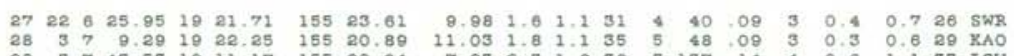

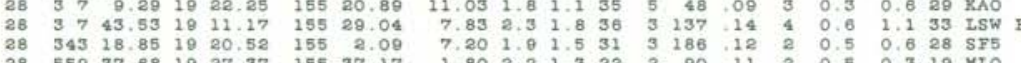

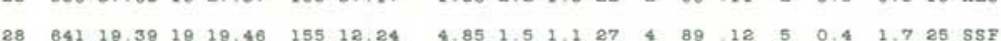

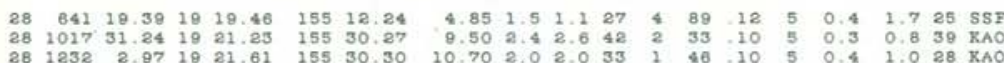

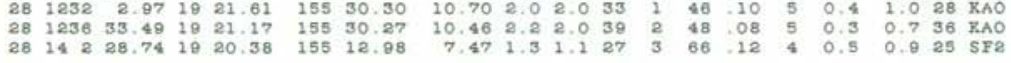

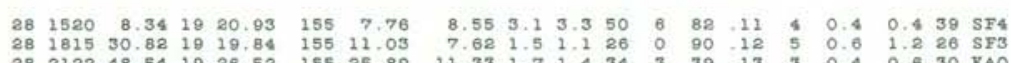

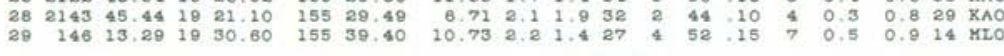

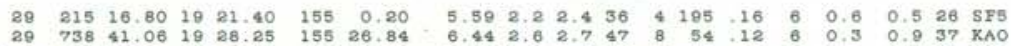

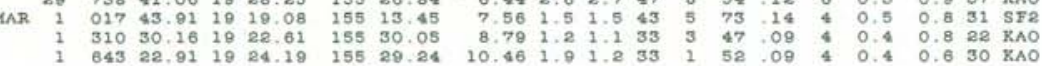

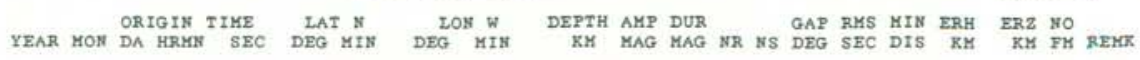

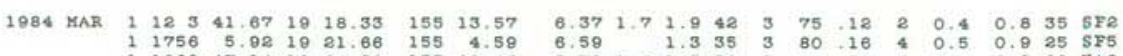

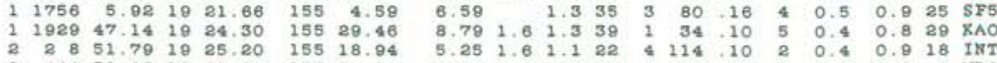

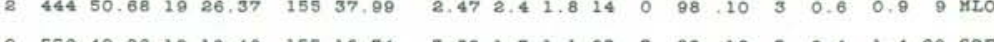

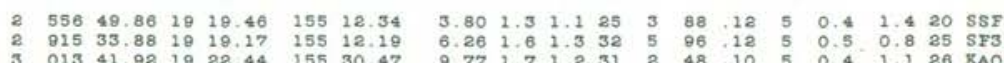

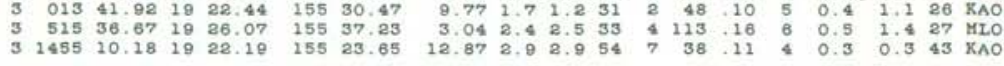
\begin{tabular}{lllllllllllllllllllll}
3 & 1629 & 34.94 & 19 & 23.88 & 155 & 26.07 & 5.76 & 1.7 & 1.3 & 30 & 3 & 36 & 12 & 3 & 0.4 & 1.0 & 20 & $\mathrm{KAO}$ \\
3 & 1720 & 23.38 & 19 & 20.15 & 155 & 12.02 & 5.24 & 2.1 & 2.1 & 41 & 7 & 78 & 15 & 5 & 0.4 & 1.0 & 29 & SF3 \\
\hline
\end{tabular}

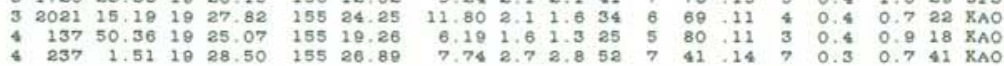

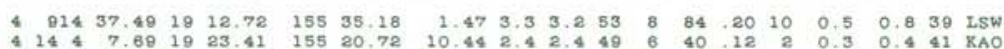

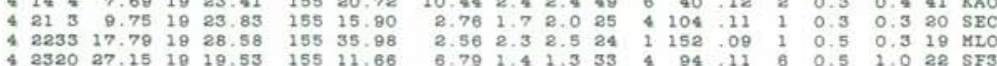
$\begin{array}{llllllllllllllllllll}5 & 258 & 42.74 & 19 & 25.76 & 155 & 21.37 & 8.56 & 1.7 & 1.3 & 35 & 6 & 80 & 13 & 4 & 0.4 & 0.7 & 24 & \text { KAO } \\ 5 & 410 & 2.98 & 10 & 22.18 & 155 & 2.23 & 7.91 & 2.78 & 1.4 & 36 & 3 & 134 & 14 & 4 & 0.7 & 0.8 & 29 & \text { SFF }\end{array}$

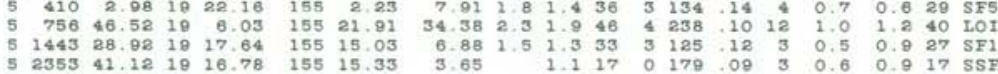

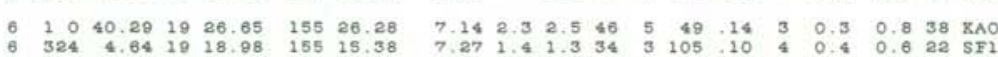

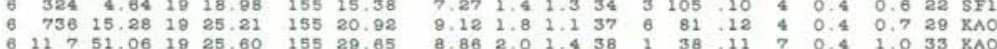

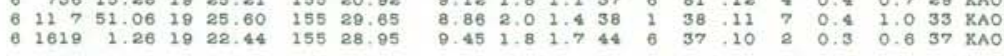

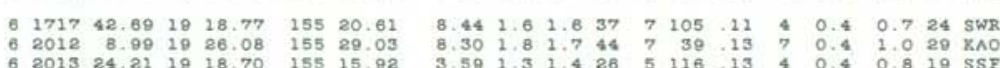

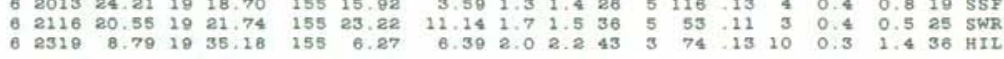
$\begin{array}{llllllllllllllllllll}7 & 1 & 0 & 29.63 & 19 & 25.58 & 155 & 38.14 & 0.03 & 1.8 & 2.4 & 24 & 3 & 101 & 30 & 5 & 0.7 & 0.9 & 11 & \text { MLO } \\ 7 & 623 & 8.65 & 10 & 11.51 & 155 & 28.52 & 7.06 & 2.1 & 2.4 & 44 & 7 & 120 & 14 & 4 & 0.5 & 0.7 & 32 & \text { LSW }\end{array}$

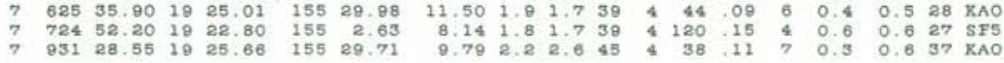

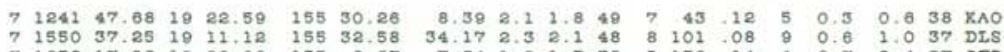

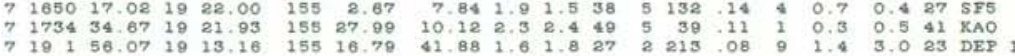




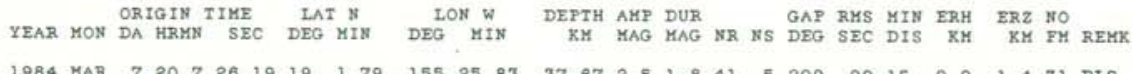

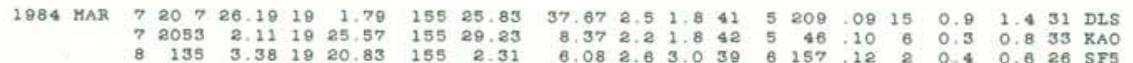

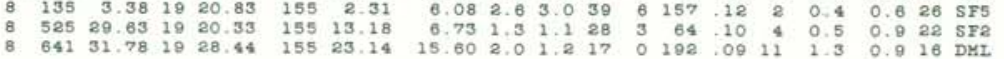

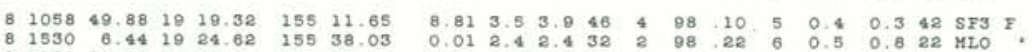

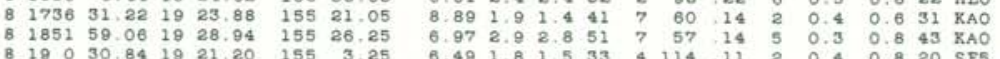

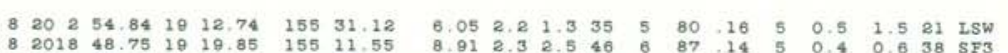

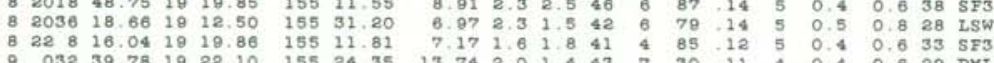

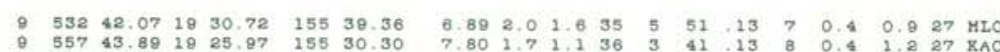

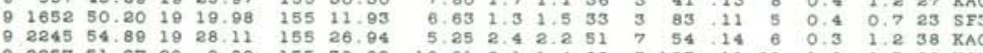

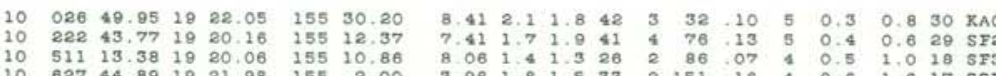

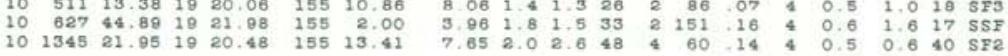

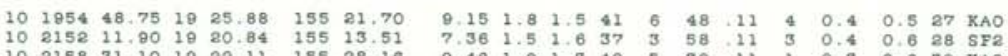

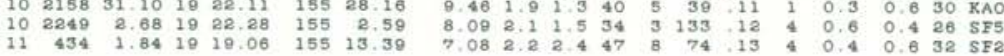

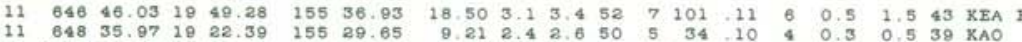

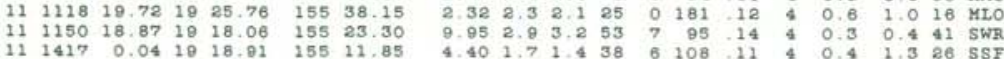

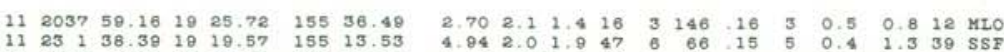

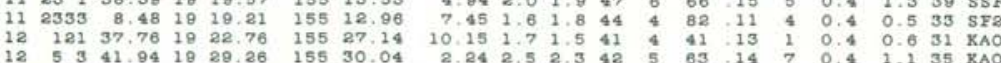

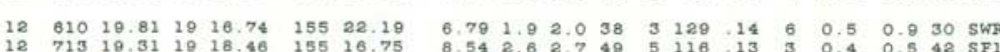

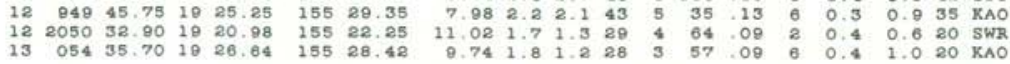

1084 HVO EARTHQUAKE SUMYARY LIST

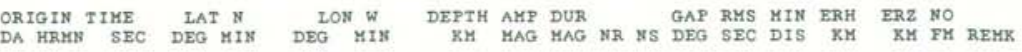
YEAR MON DA HRMS SRC DEO

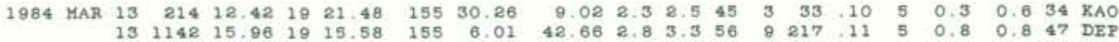

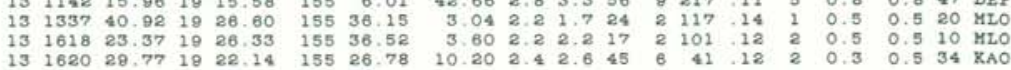

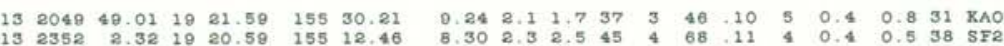

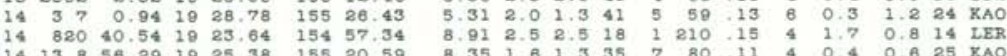

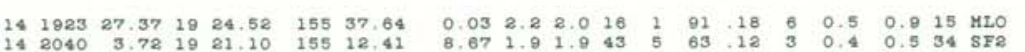

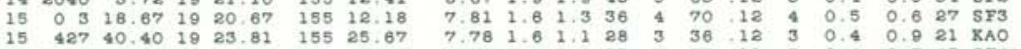

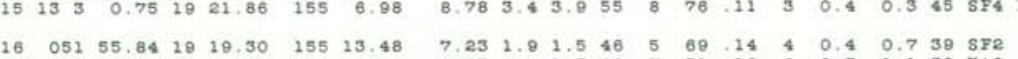

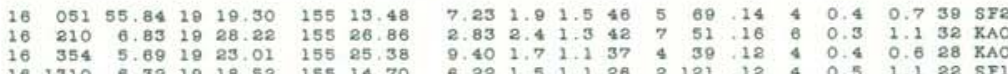

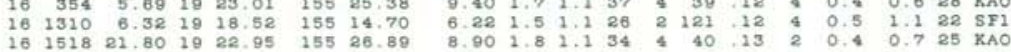

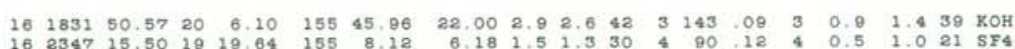

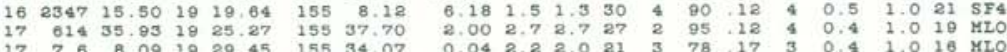

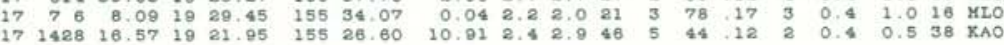

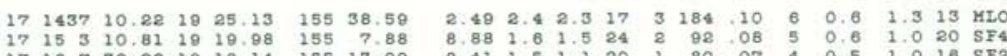

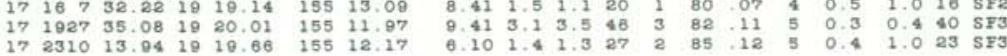

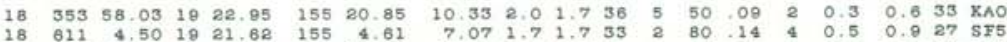

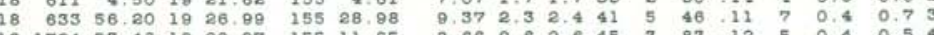

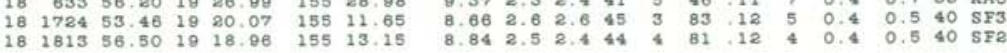

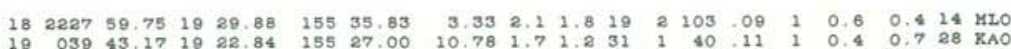

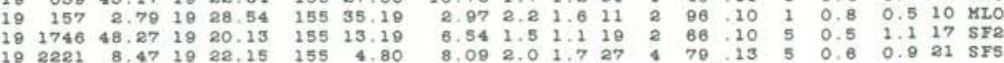

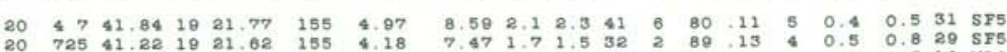

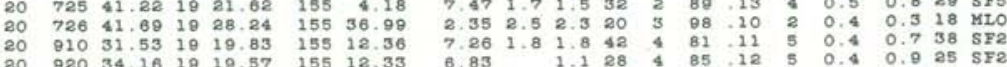




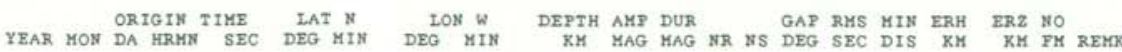

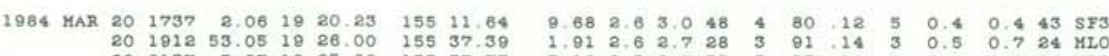

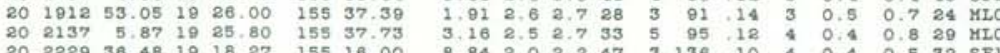

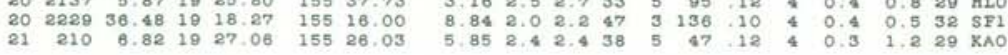

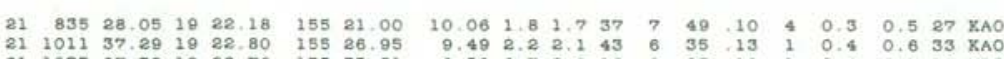

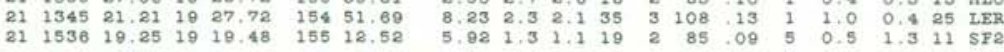

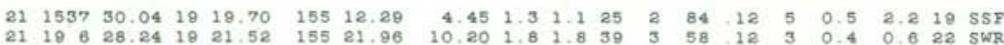

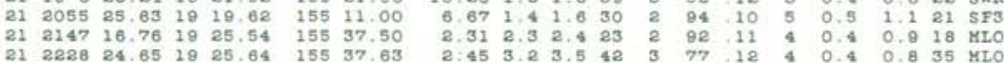

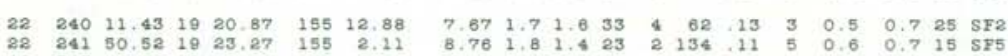

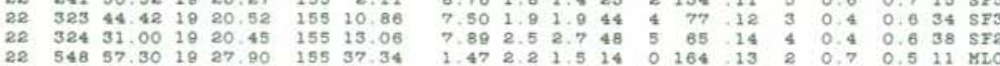

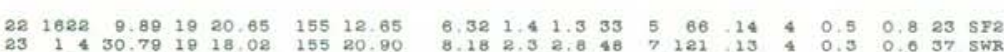

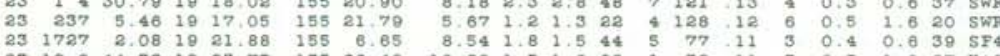

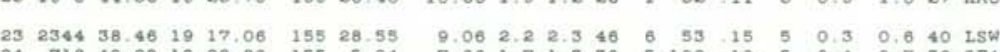

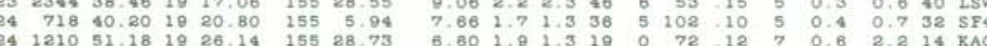

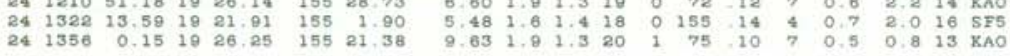

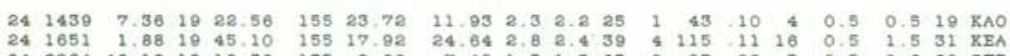

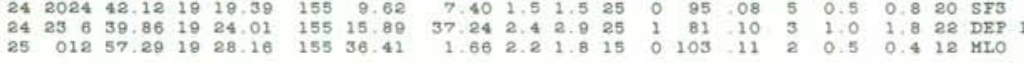

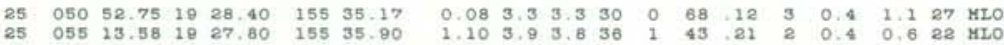

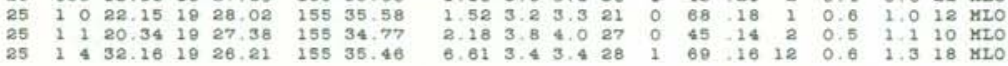

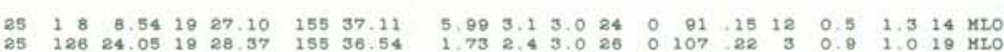

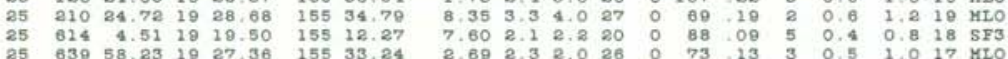

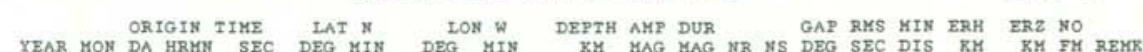
$\begin{array}{rlllllllllllllllllllllll}1984 \text { MAR } 25 & 847 & 22.71 & 19 & 19.86 & 155 & 12.92 & 7.30 & 1.8 & 1.5 & 19 & 0 & 73 & 05 & 5 & 0.4 & 0.6 & 17 & \text { SF2 } \\ 25 & 953 & 58.94 & 19 & 23.81 & 155 & 20.51 & 9.38 & 2.0 & 1.1 & 10 & 0 & 78 & 11 & 6 & 0.5 & 1.1 & 13 & \text { KAO }\end{array}$

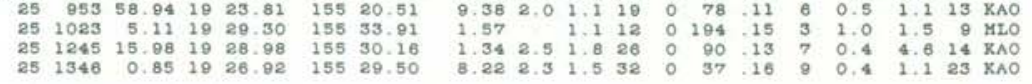

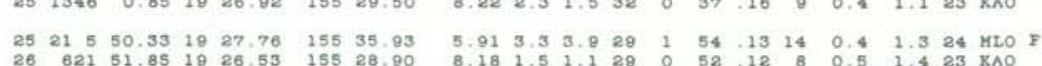

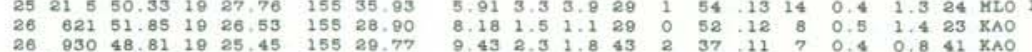

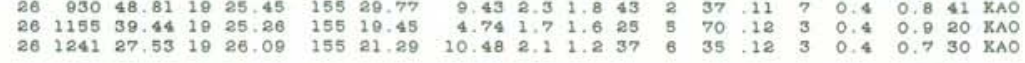

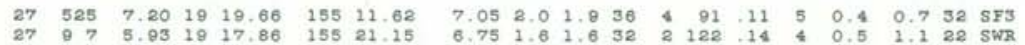

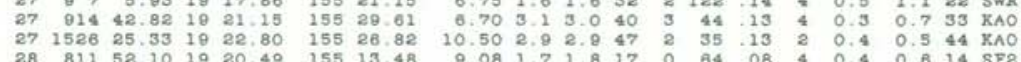

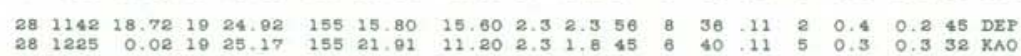

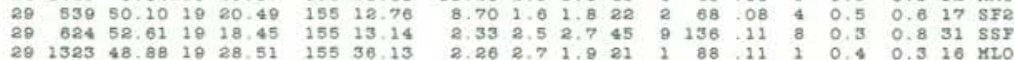

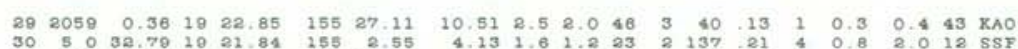

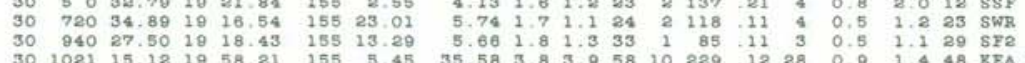

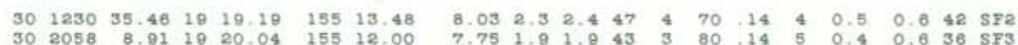

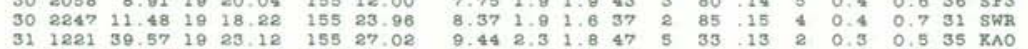

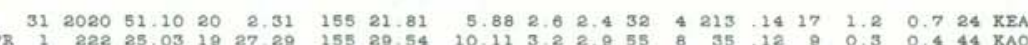

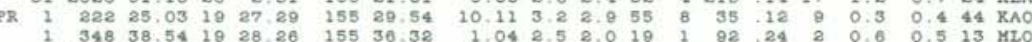

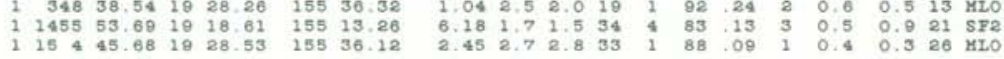

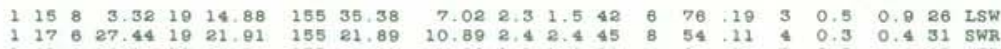

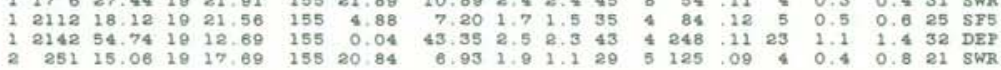
$\begin{array}{lllllllllllllllllll}2 & 517 & 42.68 & 19 & 17.61 & 155 & 12,85 & 5.31 & 1.3 & 1.1 & 18 & 2 & 132 & 07 & 2 & 0.5 & 1.1 & 14 & \text { SF2 } \\ 2 & 1819 & 8.58 & 19 & 25.68 & 154 & 55.90 & 8.18 & 2.0 & 1.4 & 27 & 2 & 162 & 13 & 4 & 0.8 & 1.0 & 20 & \text { LER }\end{array}$

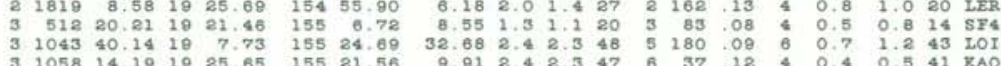




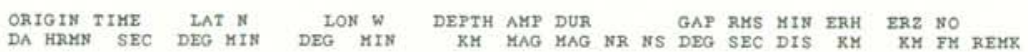

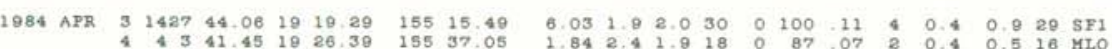

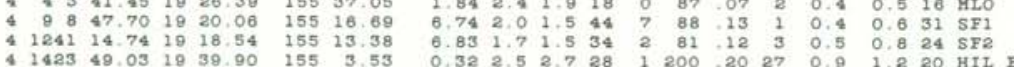

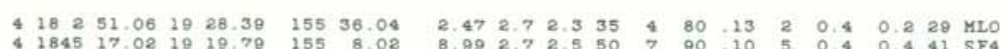

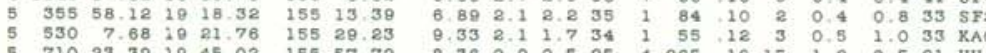
$\begin{array}{lllllllllllllllllll}5 & 1539 & 47.90 & 19 & 21.43 & 155 & 2.20 & 6.55 & 2.1 & 2.4 & 42 & 5 & 145 & 15 & 3 & 0.4 & 0.6 & 38 & 5 F\end{array}$ $\begin{array}{lll} & \\ 5 & & \end{array}$

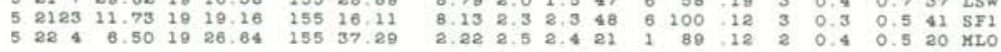

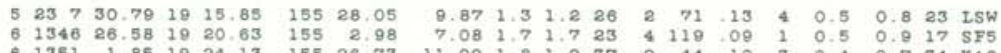

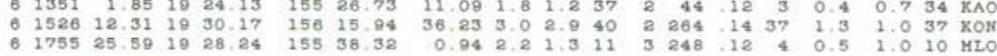

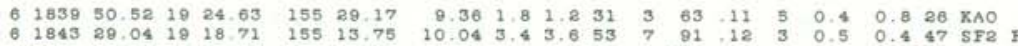

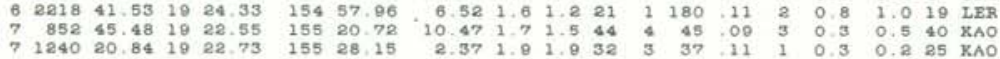

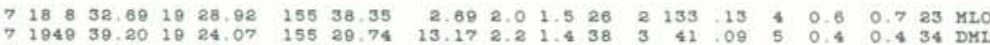

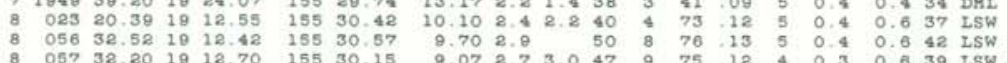

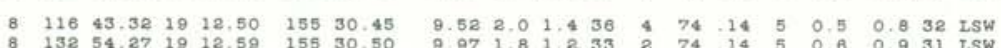

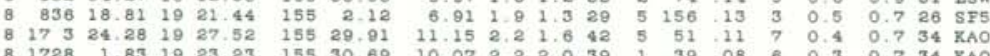

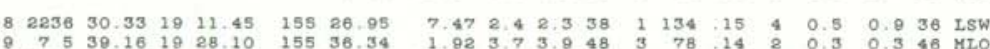

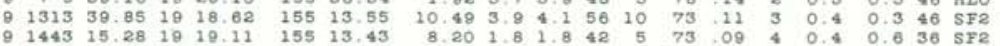

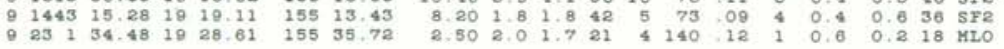

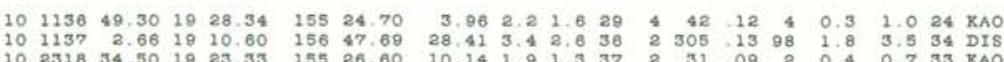

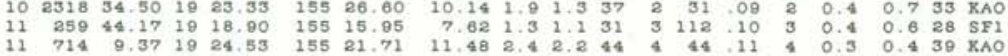

1984 HVO EARTHQUAKE SUMHARY LIST

PAGE 18

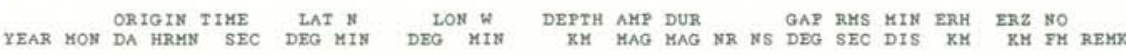
1984 APR

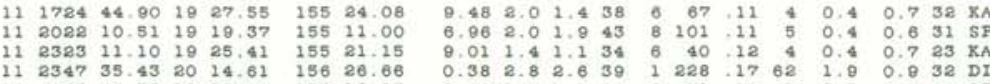

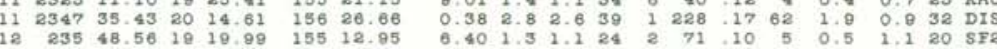

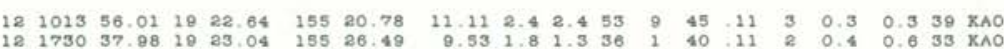

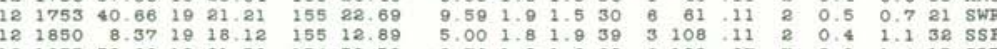

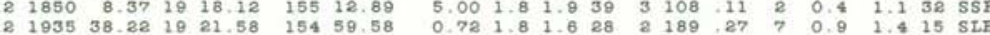
$\begin{array}{rrrrrrrrrrrrrrrrrrr}13 & 316 & 39.24 & 19 & 23.19 & 155 & 1.05 & 8.08 & 1.8 & 1.5 & 35 & 2 & 144 & 17 & 5 & 0.8 & 0.6 & 27 & \text { SP5 } \\ 13 & 2350 & 46.72 & 10 & 19.13 & 155 & 11.31 & 8.24 & 1.9 & 2.2 & 40 & 6 & 106 & 11 & 5 & 0.4 & 0.7 & 34 & \text { SF3 }\end{array}$

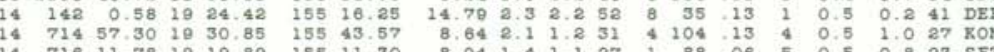

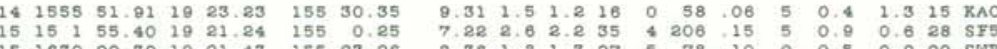

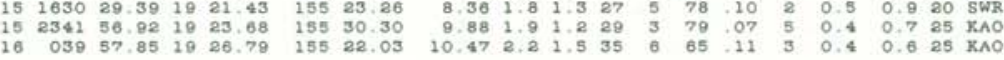

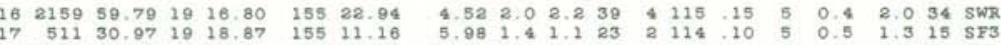

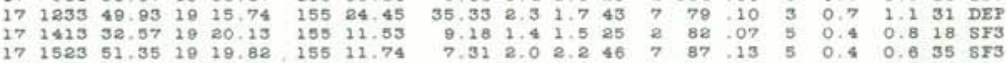

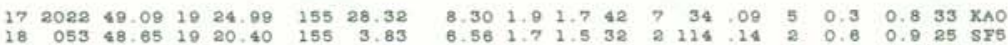

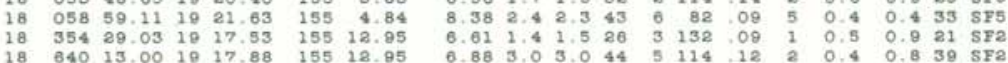

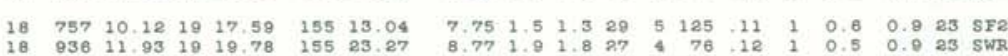

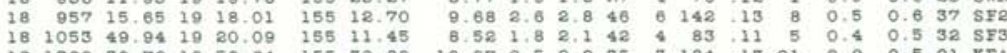

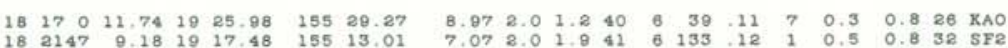

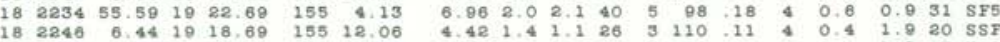

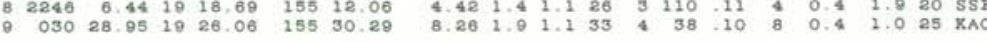

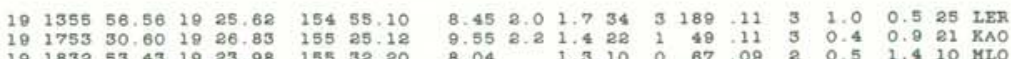

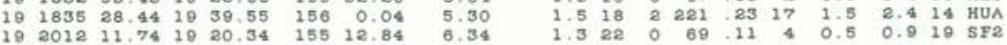




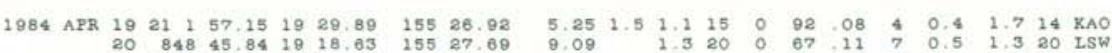

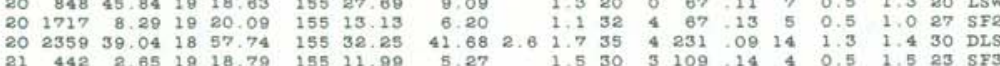

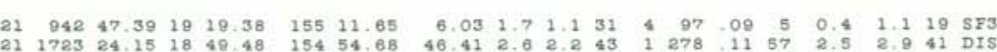

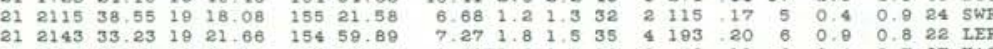

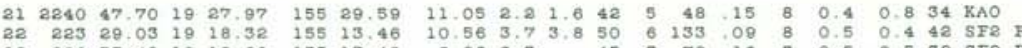

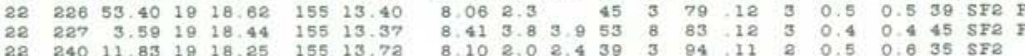

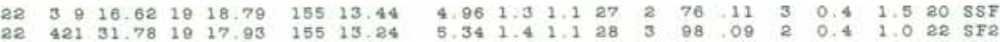

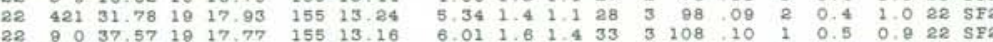

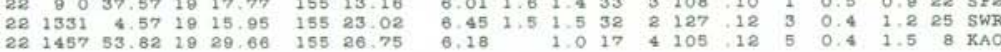

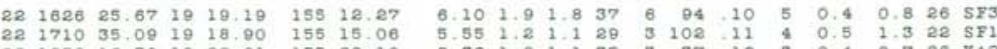

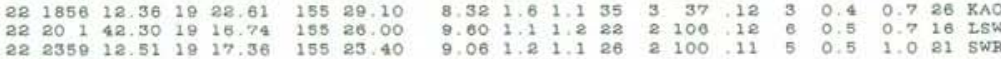

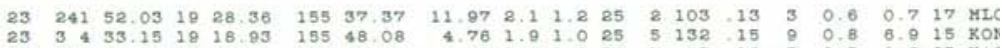

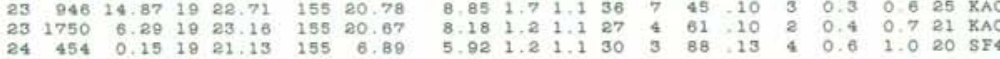

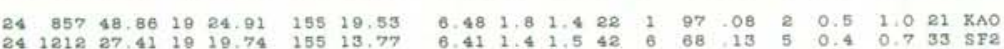

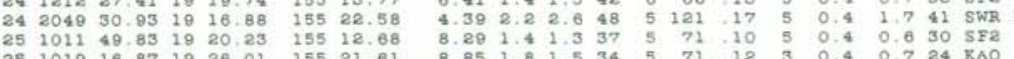

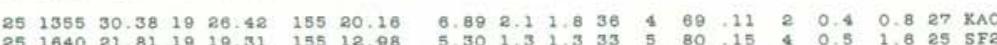

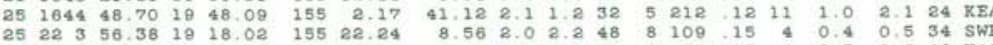

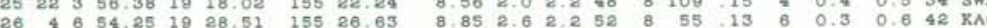

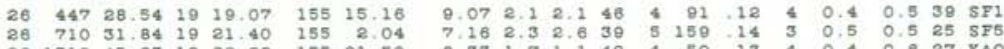

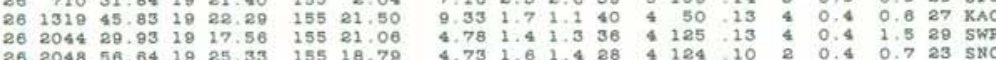

1984 HVO EARTHQUARE SUMMARY LIST

PAGE 20

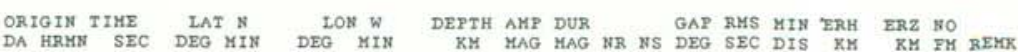

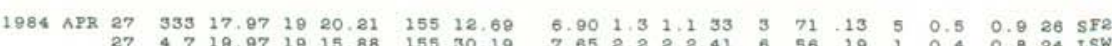

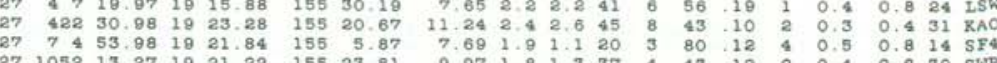

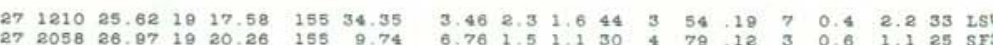

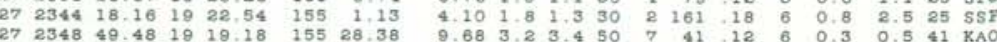

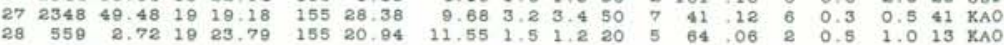

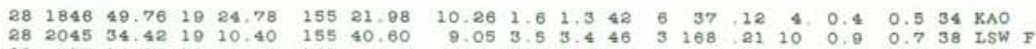

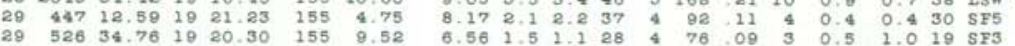

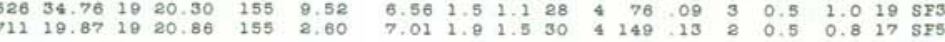

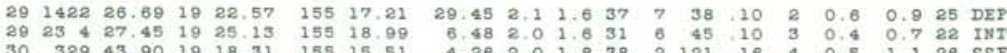

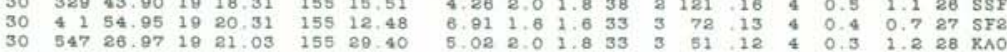
$\begin{array}{rrrrrrrrrrrrrrrrrrr}30 & 619 & 16.25 & 19 & 21.72 & 155 & 22.03 & 10.01 & 2.2 & 2.2 & 44 & 6 & 56 & 12 & 4 & 0.4 & 0.5 & 32 & \text { SWR } \\ 30 & 1349 & 34.24 & 19 & 43.42 & 154 & 52.78 & 40.40 & 3.5 & 3.0 & 48 & 5 & 234 & 12 & 22 & 0.9 & 1.4 & 42 & \text { HII }\end{array}$

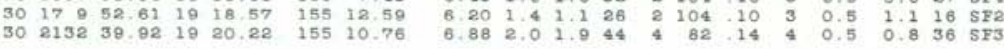

$\begin{array}{llllllllllllllllllll}\text { MAY } & 2 & 041 & 45.24 & 18 & 19.97 & 155 & 12.25 & 6.96 & 1.3 & 1.1 & 31 & 3 & 80 & 10 & 5 & 0.5 & 0.9 & 22 & 5 F 3\end{array}$

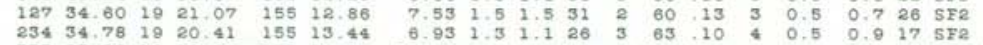

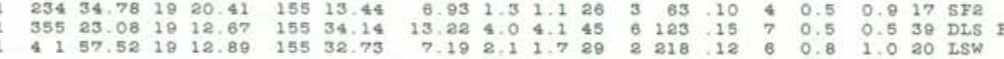

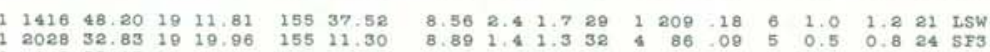

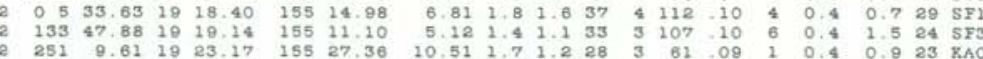

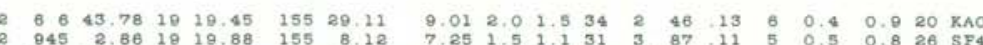

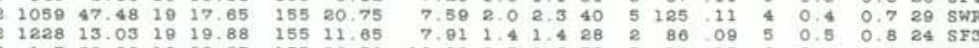

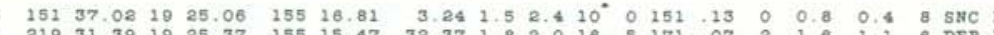

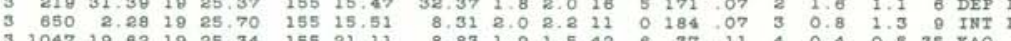

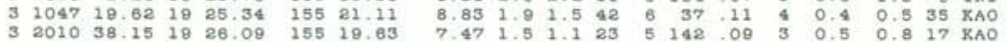




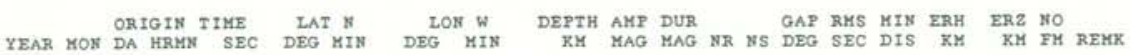

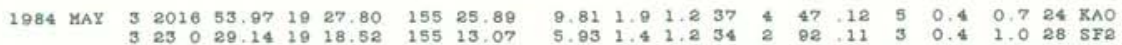

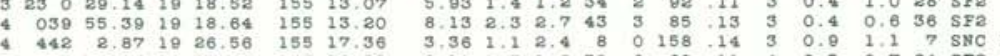

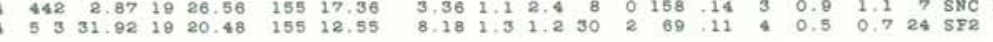

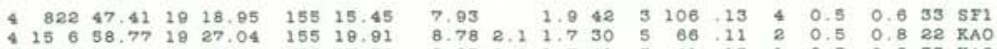

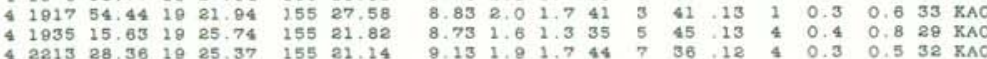

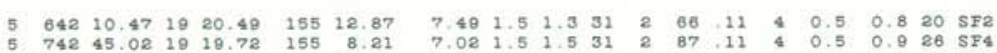

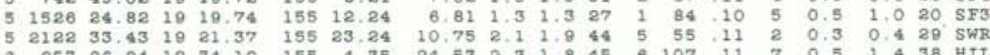

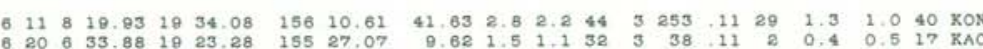

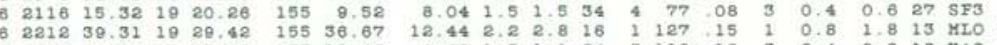

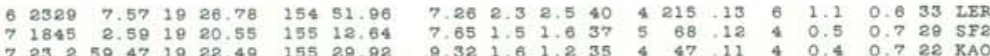

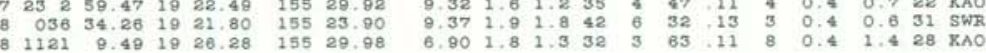
$\begin{array}{lllllllllllllllllll}8 & 1335 & 11.46 & 19 & 17.96 & 155 & 24.17 & 8.91 & 1.6 & 1.3 & 30 & 3 & 82 & 13 & 4 & 0.4 & 0.7 & 21 & \text { swR } \\ 8 & 1426 & 57.11 & 19 & 30.59 & 155 & 24.14 & 3.26 & 1.6 & 1.5 & 24 & 6 & 161 & 15 & 2 & 0.7 & 0.5 & 17 & \text { MIO }\end{array}$

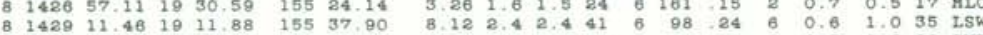

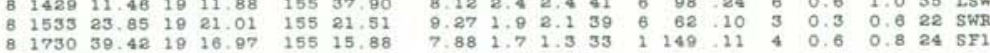

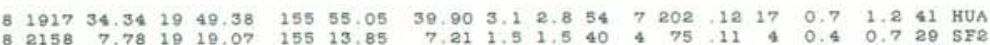

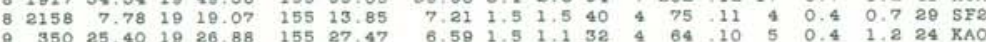

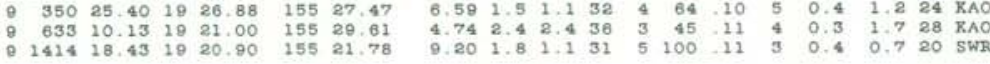

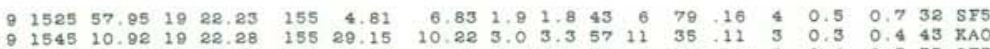

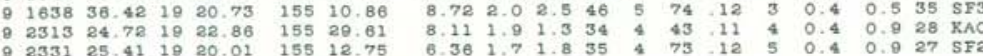

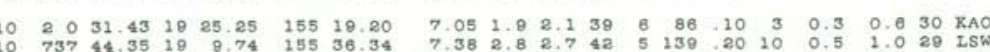

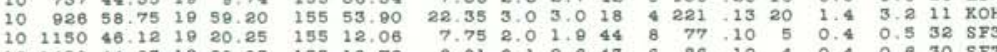

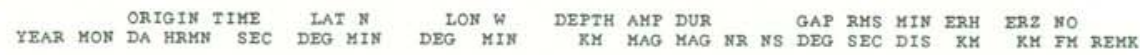

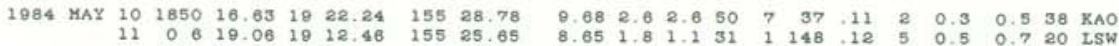

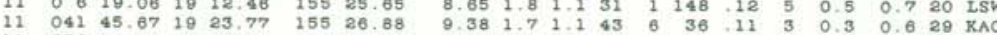

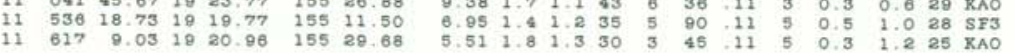

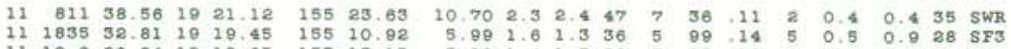

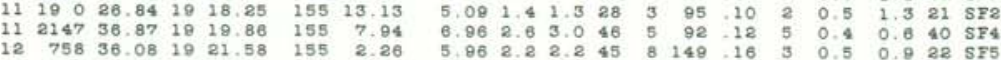

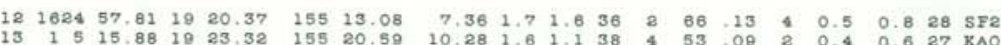

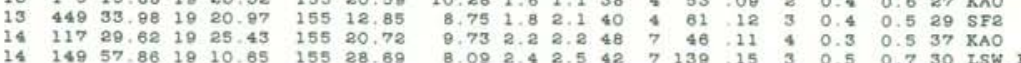

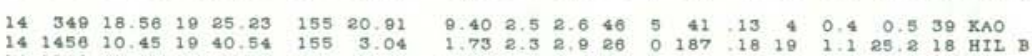

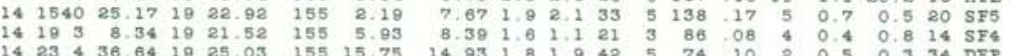

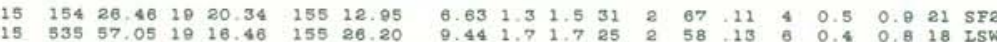

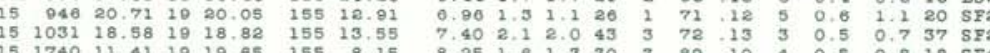

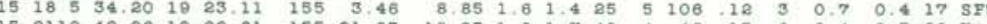

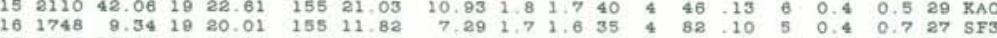

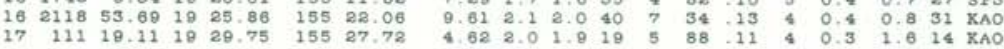

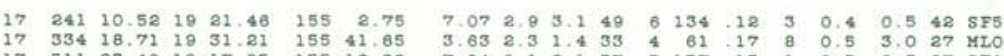

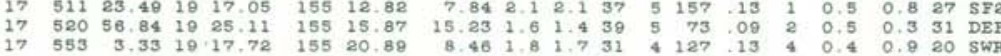

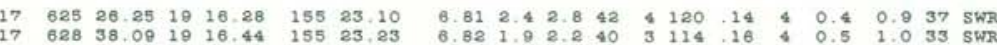

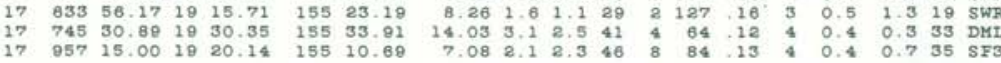

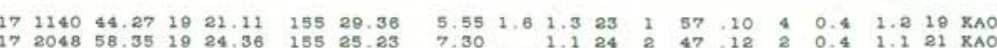

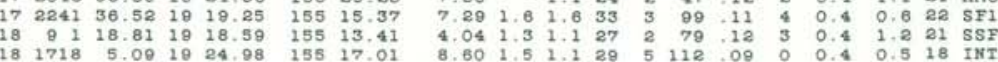




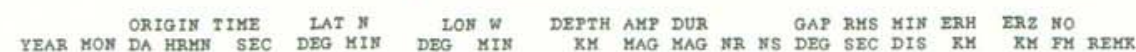

$\begin{array}{rllllllllllllllllllll}1984 \mathrm{BAY} & 18 & 1021 & 22.94 & 19 & 24.01 & 155 & 26.74 & 9.40 & 1.8 & 1.5 & 39 & 4 & 35 & 12 & 3 & 0.4 & 0.6 & 28 & \mathrm{KAO} \\ 18 & 21 & 43.10 & 10 & 17.49 & 155 & 25.75 & 9.51 & 2.3 & 2.7 & 51 & 6 & 60 & 10 & 7 & 0.5 & 0.6 & 40 & \text { ISW }\end{array}$

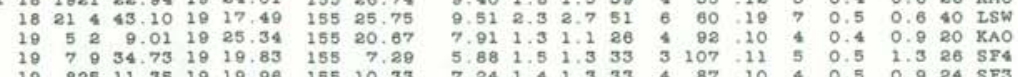

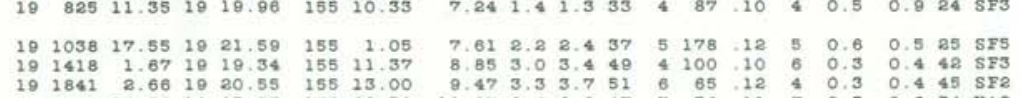

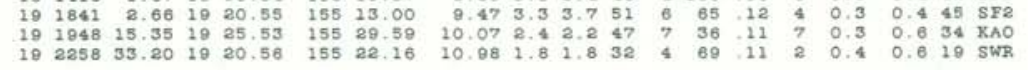

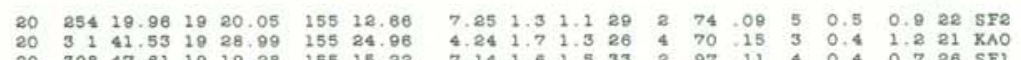

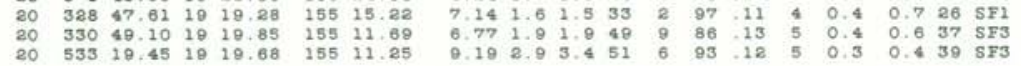

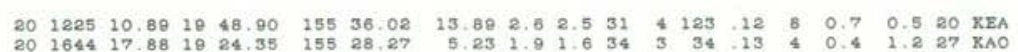

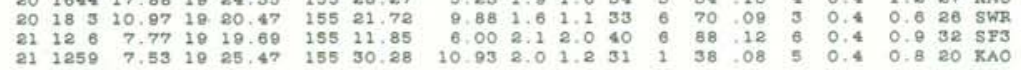

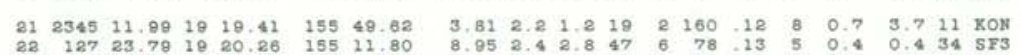

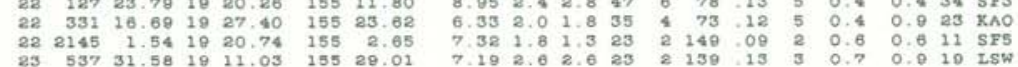

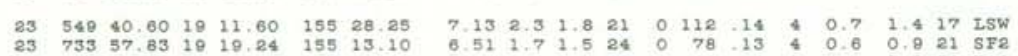

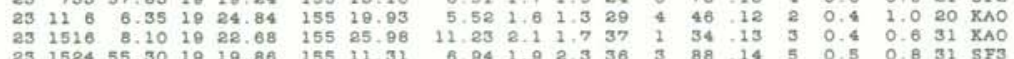

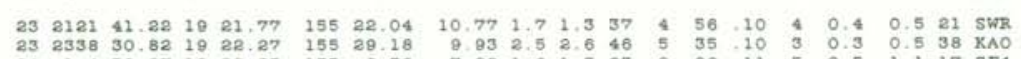

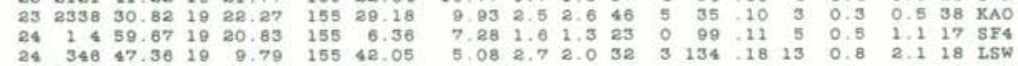

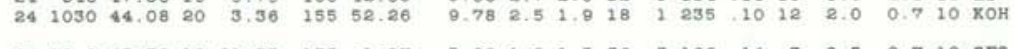

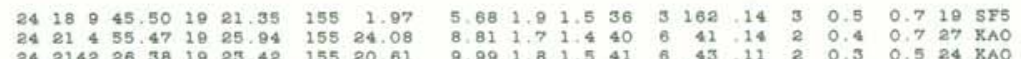

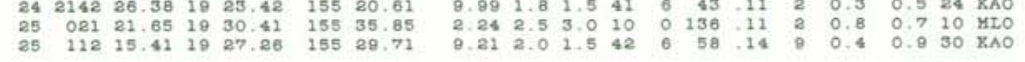

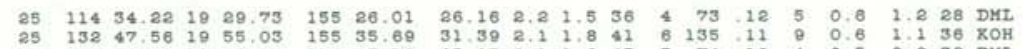

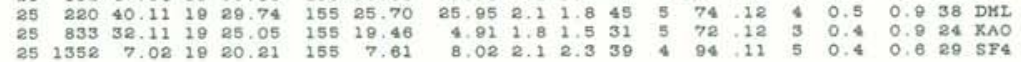

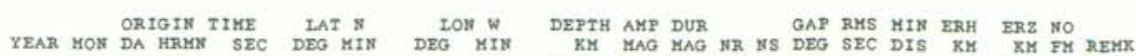

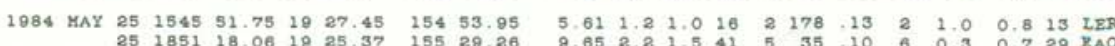

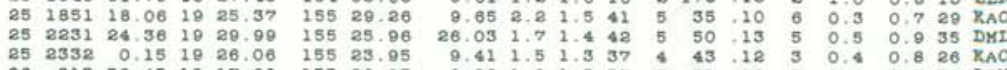
(20.7.

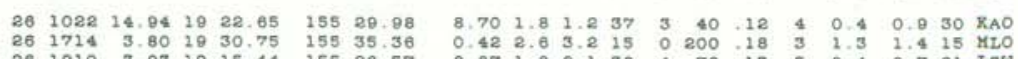

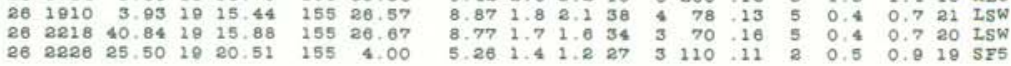

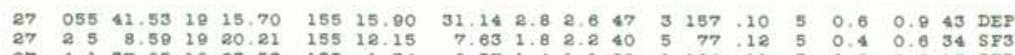

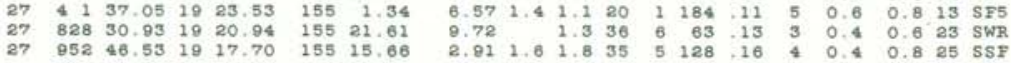

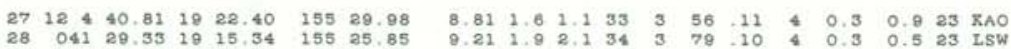

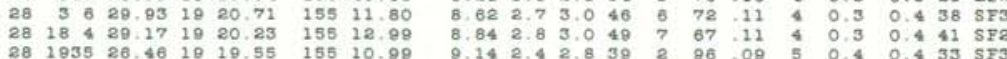

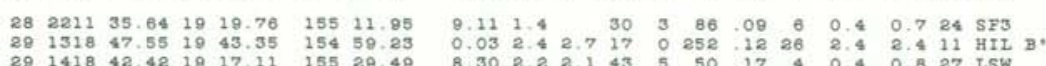

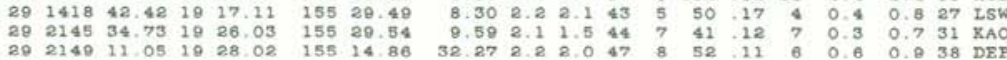

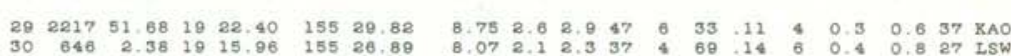

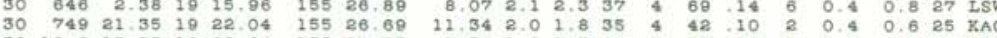

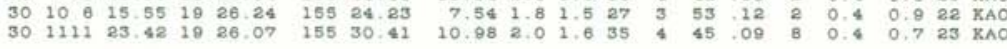

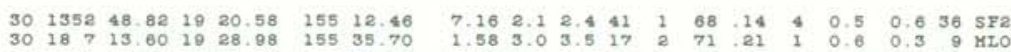

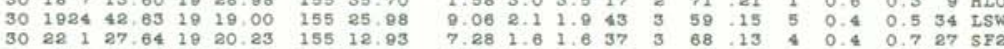

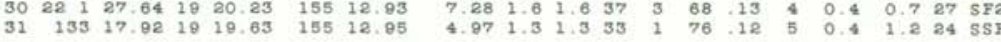

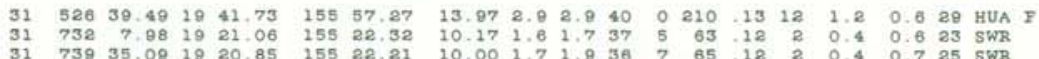

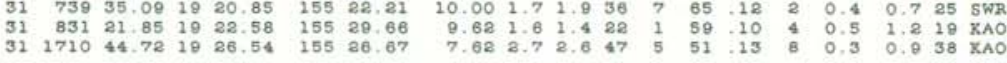

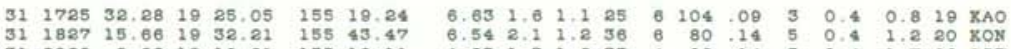

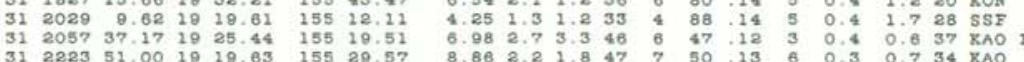




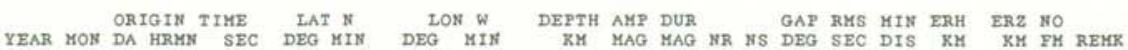

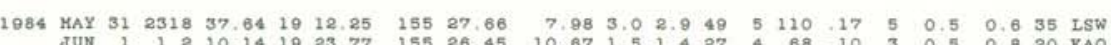

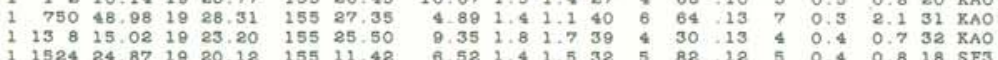

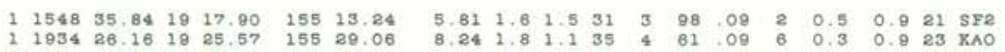

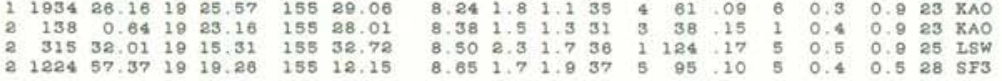

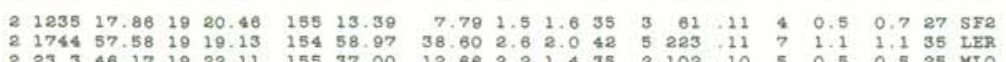

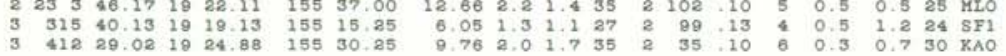

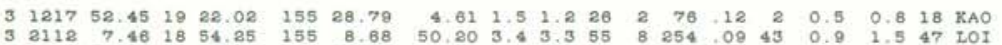

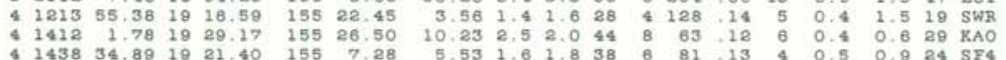

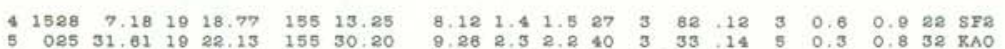

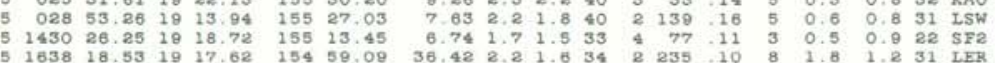

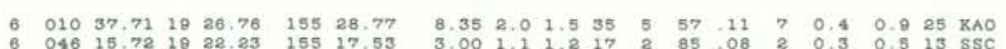

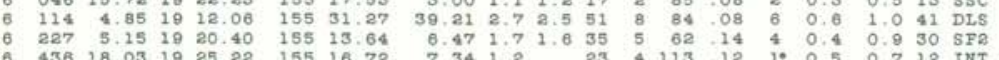

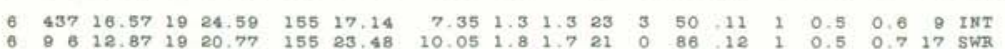

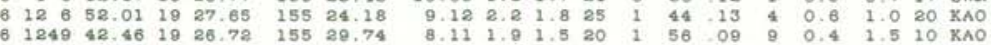

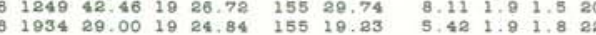

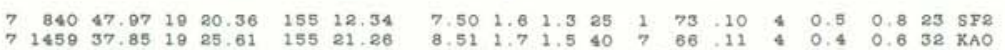

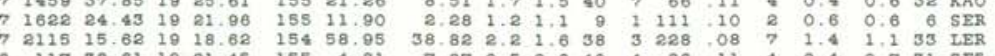

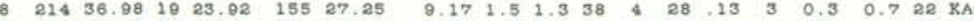

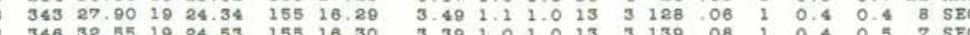

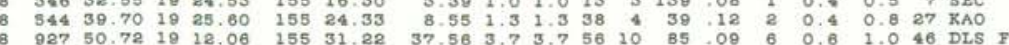

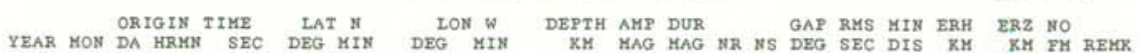
1984 JUS $8 \begin{array}{rlllllllllllllllllll}933 & 12.30 & 19 & 12.10 & 155 & 31.20 & 37.45 & 3.1 & 3.3 & 57 & 10 & 83 & .10 & 6 & 0.6 & 0.9 & 47 & \text { DLS }\end{array}$

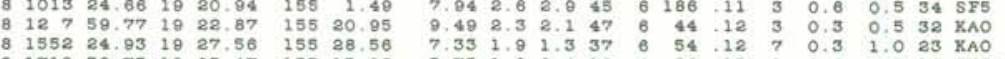

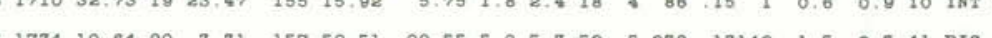

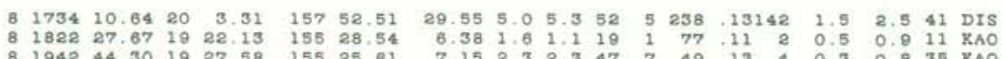

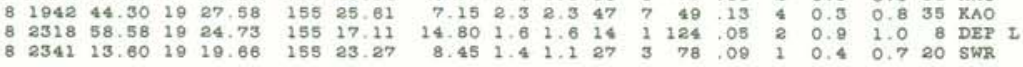

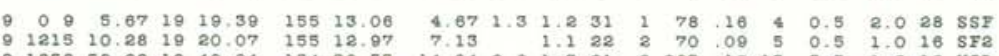

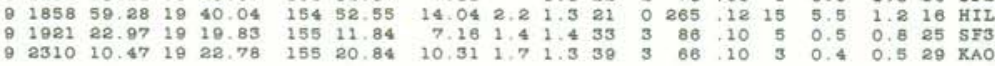
$\begin{array}{rrrrrrrrrrrrrrrrrrr}10 & 223 & 50.52 & 19 & 18.23 & 154 & 58.69 & 39.42 & 2.2 & 1.6 & 35 & 2 & 232 & 08 & 8 & 1.6 & 1.5 & 32 & \text { LER } \\ 10 & 323 & 33.38 & 19 & 18.21 & 155 & 13.59 & 7.84 & 2.1 & 2.3 & 41 & 5 & 74 & 11 & 2 & 0.4 & 0.6 & 28 & \text { SFE }\end{array}$

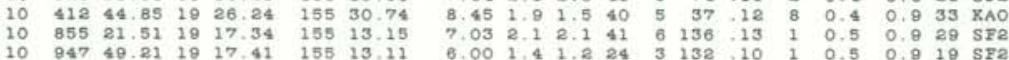

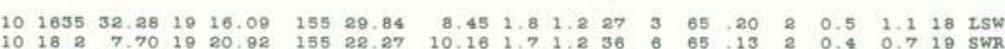

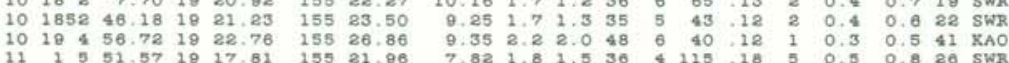

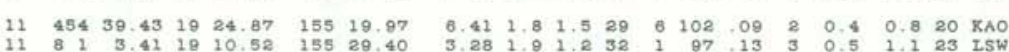

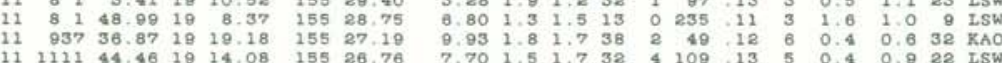

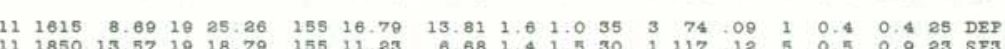

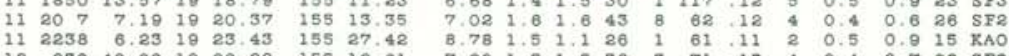

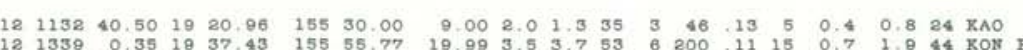

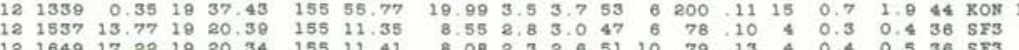

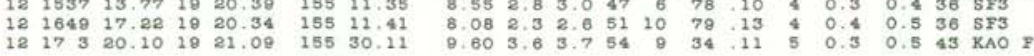

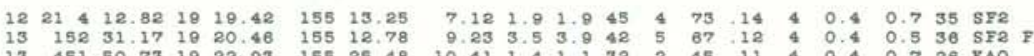

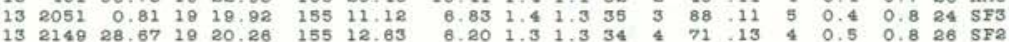


1984 HVO EARTHQUAKE SUMMARY LIST

PAGE 27

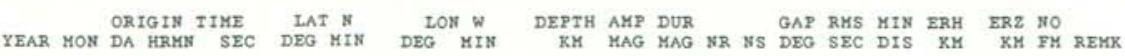

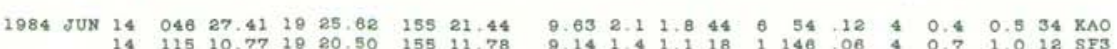

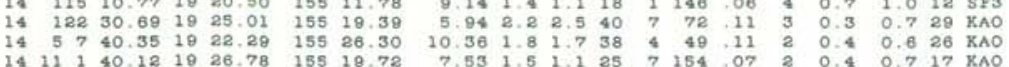

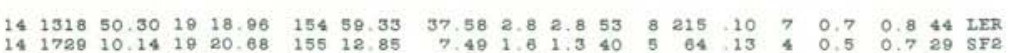

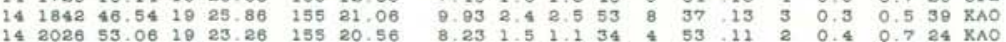

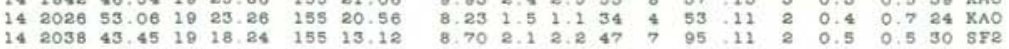
$\begin{array}{rrrrrrrrrrrrrrrrrrrr}14 & 2328 & 40.04 & 19 & 17.17 & 155 & 22.02 & 6.30 & 1.5 & 1.1 & 24 & 1 & 124 & 10 & 6 & 0.4 & 1.1 & 18 & \text { SWR } \\ 15 & 310 & 31 & 36 & 19 & 18.70 & 155 & 17.68 & 12.43 & 1.9 & 1.6 & 50 & 10 & 115 & 11 & 2 & 0.4 & 0.4 & 38 & \text { SWR }\end{array}$

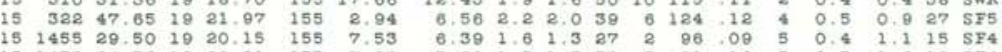

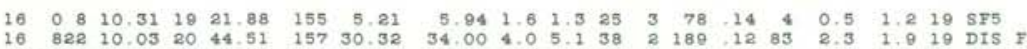

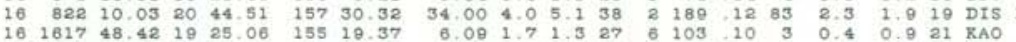

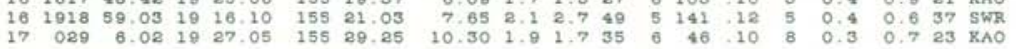

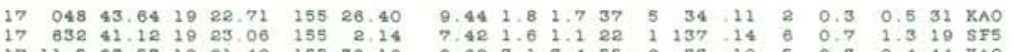

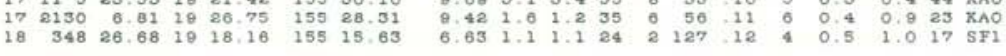
$\begin{array}{lllllllllllllllllllll}18 & 6 & 3 & 33.02 & 18 & 20.40 & 155 & 30.91 & 8.93 & 2.0 & 1.5 & 32 & 4 & 55 & 11 & 7 & 0.4 & 1.1 & 23 & \text { KAO } \\ 18 & 1341 & 18.14 & 19 & 20.94 & 155 & 12.77 & 8.42 & 2.0 & 2.3 & 42 & 6 & 62 & 13 & 3 & 0.4 & 0.5 & 29 & \text { SF2 }\end{array}$

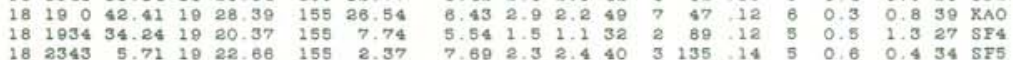

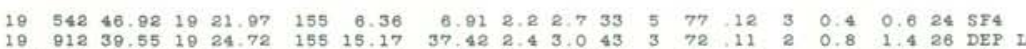

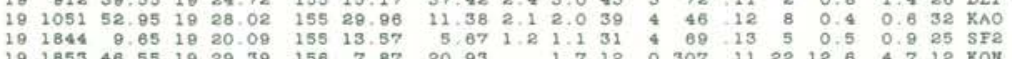

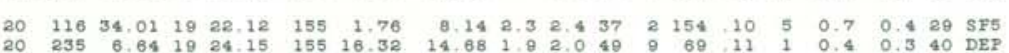

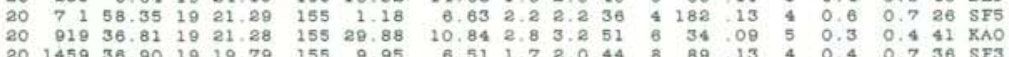

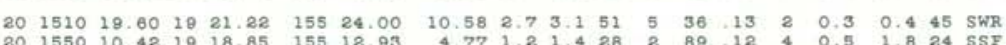

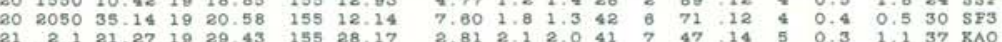

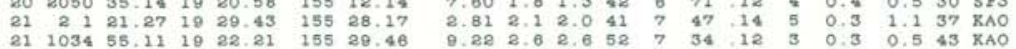

1984 HVO EARTHQUARE SUMMARY LIST

PAGE 28

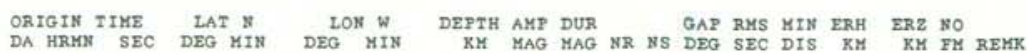

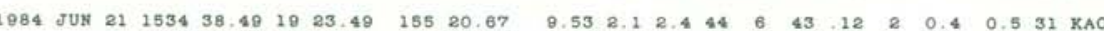

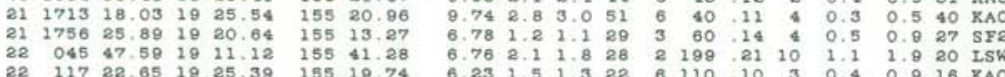

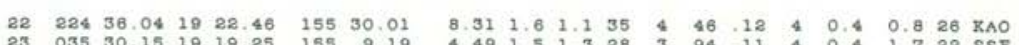

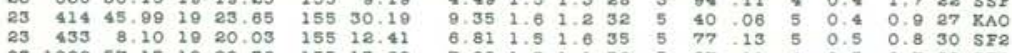

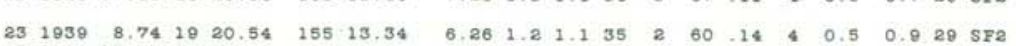

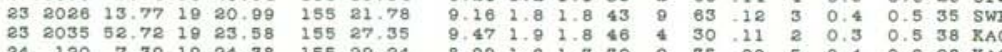

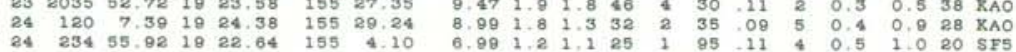

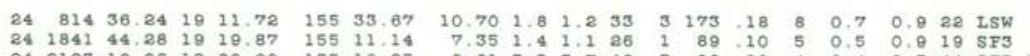

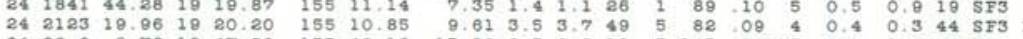

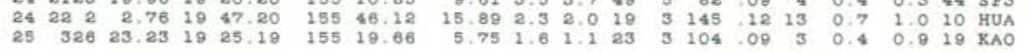

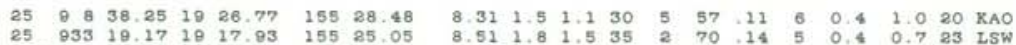

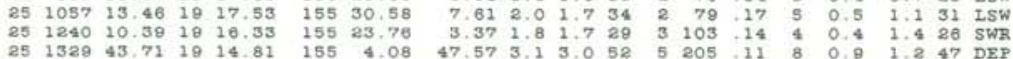

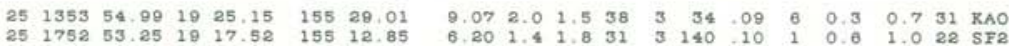

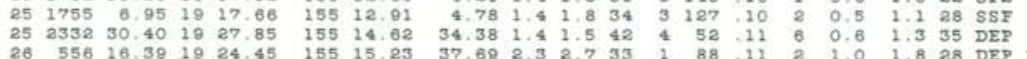

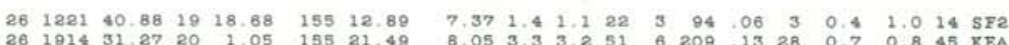

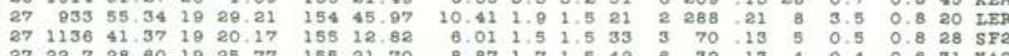

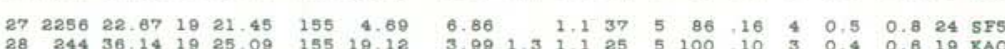

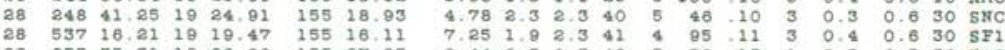

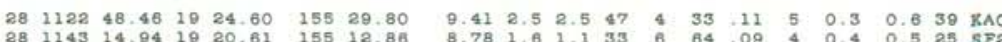

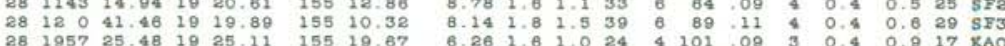

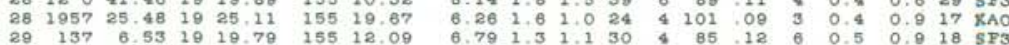




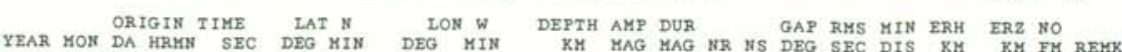

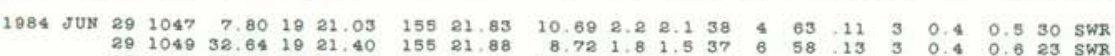

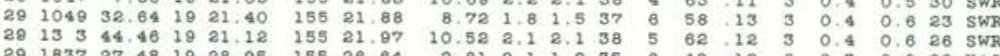

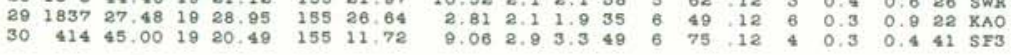

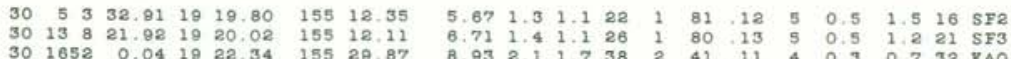

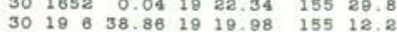

JUL 1

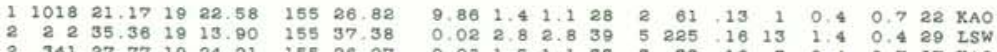

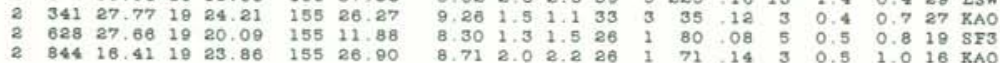

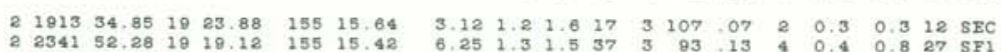

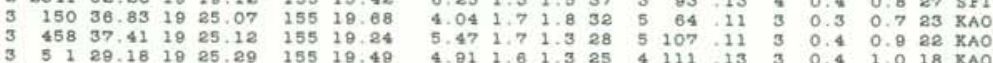

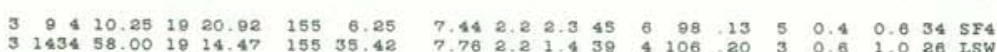

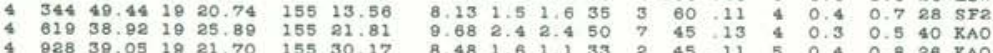

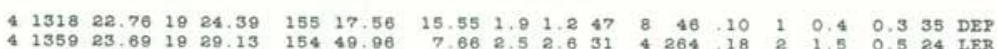

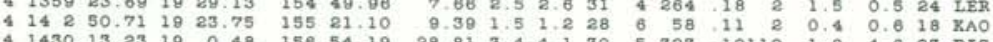

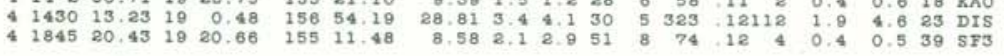

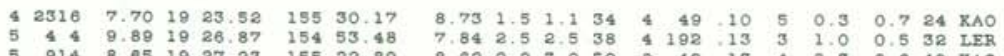

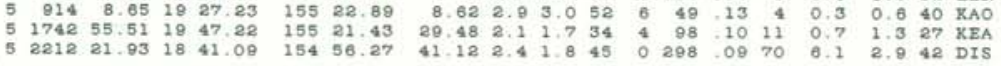

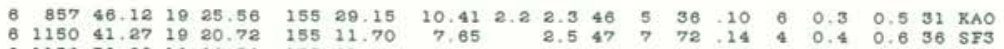

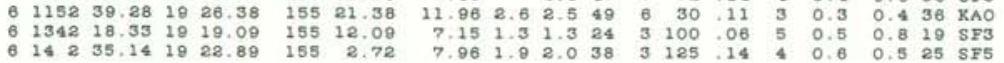

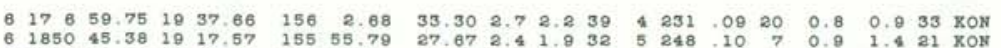

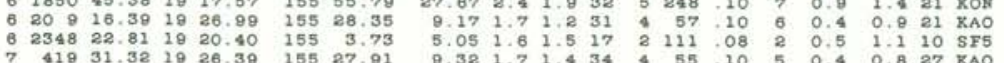

1984 HVO EARTHQUAKE SUMHARY LIST

PAGE 30

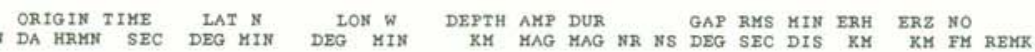

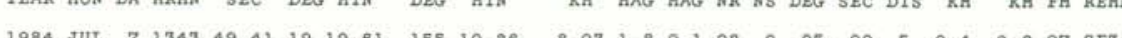

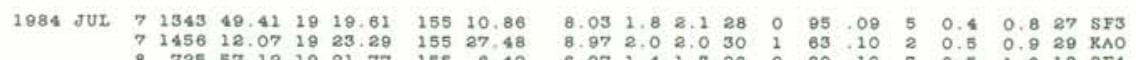

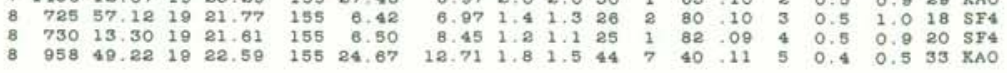

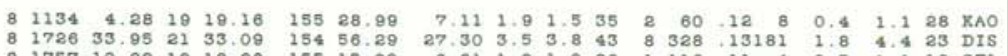

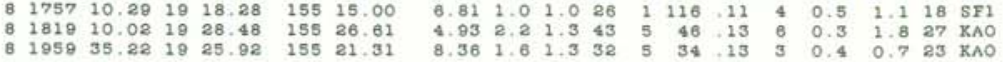

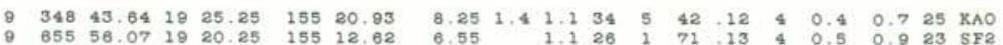

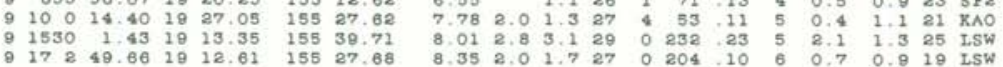

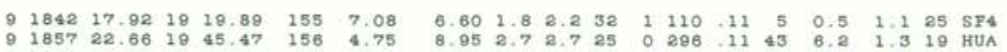

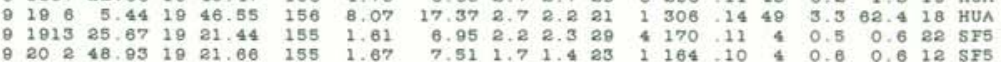

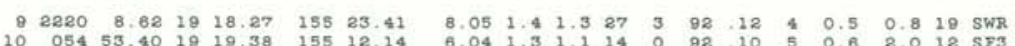

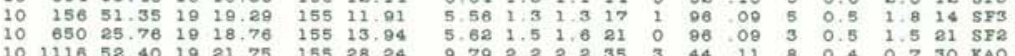

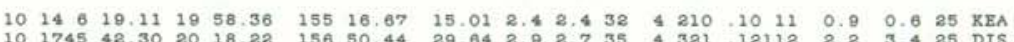

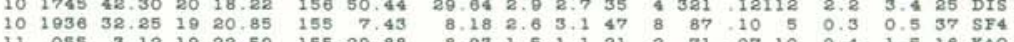

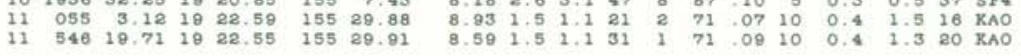

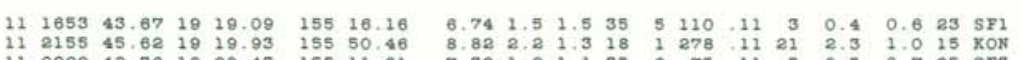

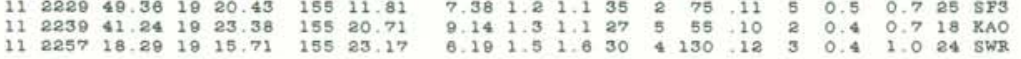
$\begin{array}{lllllllllllllllllll}12 & 254 & 58.45 & 19 & 20.62 & 155 & 6.15 & 5.51 & 1.4 & 1.1 & 19 & 2 & 106 & .12 & 5 & 0.5 & 1.3 & 8 & 5 F 4 \\ 12 & 342 & 51.22 & 10 & 19.03 & 155 & 13.28 & 4.40 & 1.7 & 1.9 & 32 & 5 & 77 & 11 & 4 & 0.4 & 1.5 & 23 & 58 Y\end{array}$

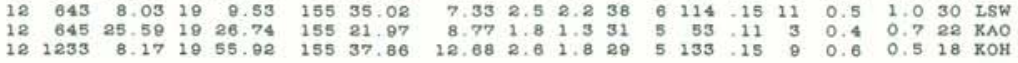
$\begin{array}{llllllllllllllllllll}12 & 1245 & 2.13 & 19 & 17.80 & 155 & 20.62 & 7.14 & 1.8 & 1.2 & 40 & 9 & 124 & 12 & 4 & 0.3 & 0.6 & 26 & \text { SWR } \\ 12 & 1315 & 40.27 & 19 & 19.47 & 155 & 11.98 & 7.56 & 1.3 & 1.3 & 32 & 3 & 91 & 10 & 5 & 0.5 & 0.8 & 23 & \text { SF3 }\end{array}$

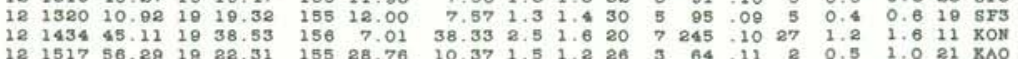




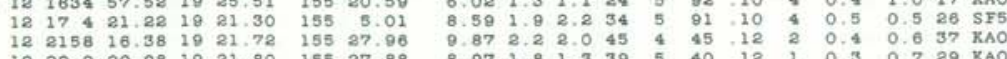

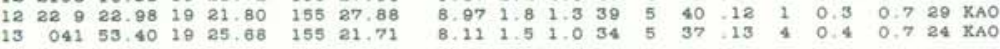
$\begin{array}{lllllllllllllllllll}13 & 045 & 1.28 & 19 & 20.26 & 155 & 20.91 & 20.37 & 1.9 & 1.7 & 43 & 6 & 71 & 13 & 4 & 0.6 & 0.9 & 31 & \text { DEP } \\ 13 & 337 & 48.48 & 10 & 24.38 & 155 & 29.23 & 0.21 & 1.9 & 1.7 & 40 & 3 & 33 & 11 & 5 & 0.3 & 0.8 & 30 & \mathrm{KAO}\end{array}$

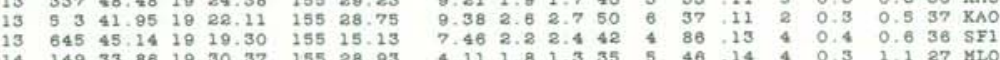

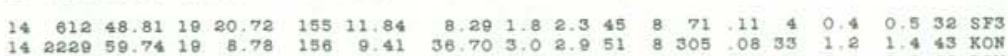

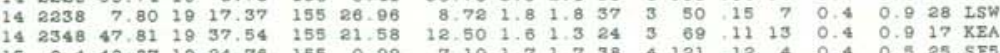

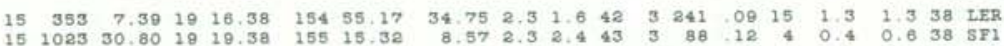

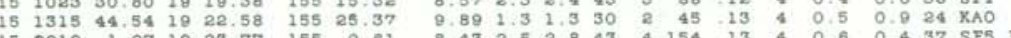

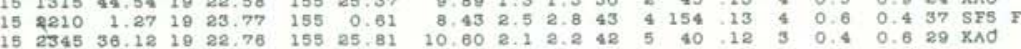

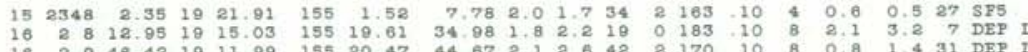

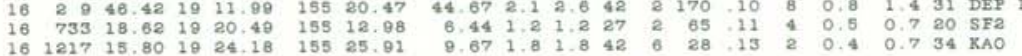

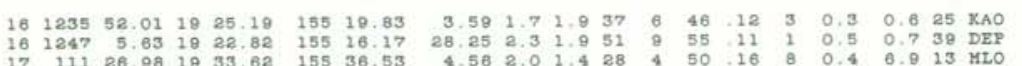

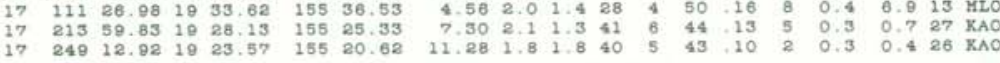

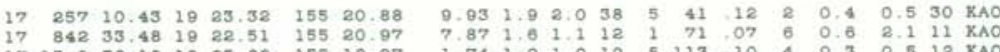

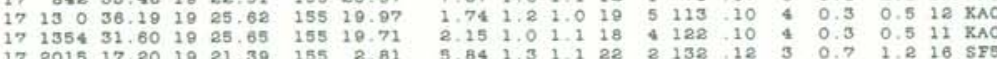

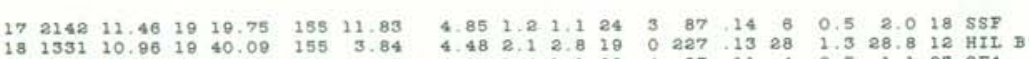

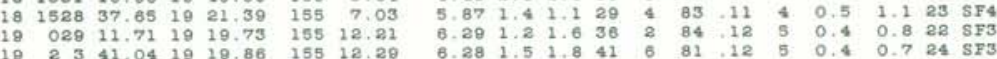

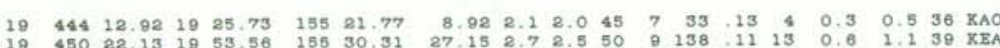

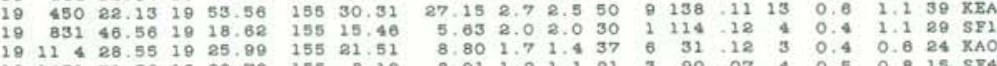

YEAR MOS ORIGIN TIME

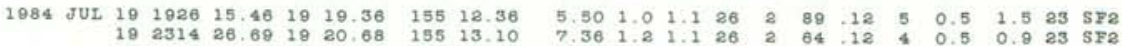

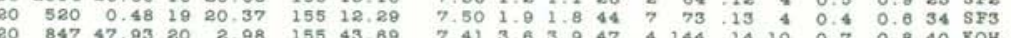

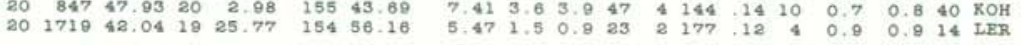

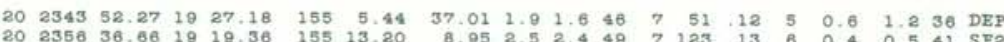

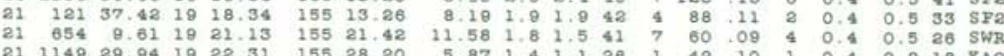

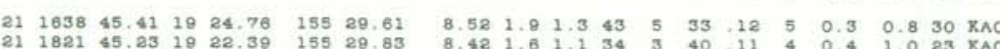

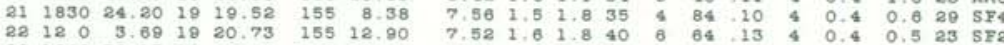

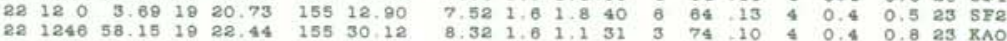

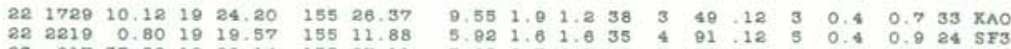

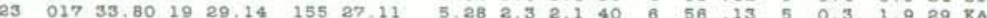

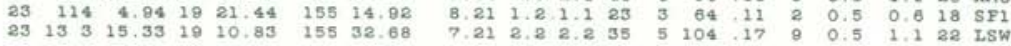

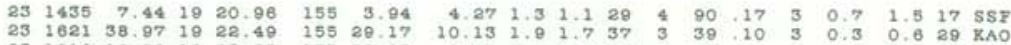

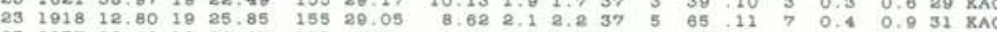

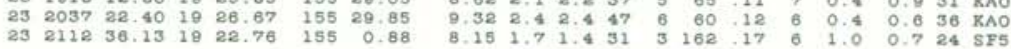

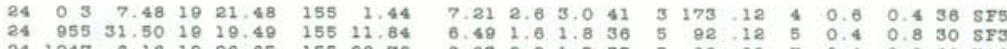

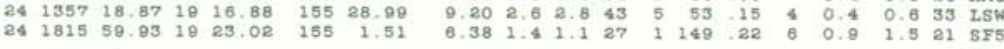

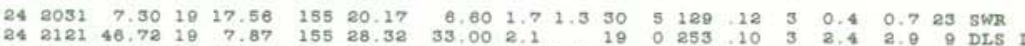

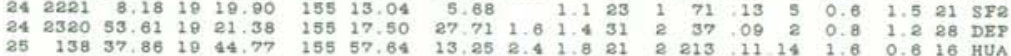

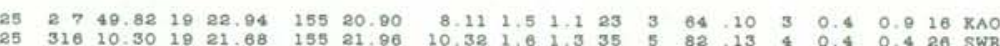

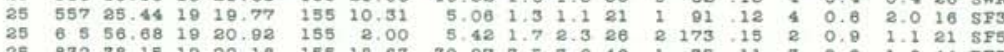

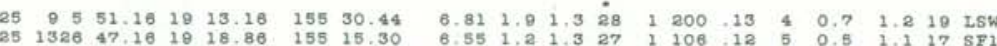

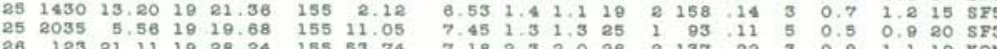




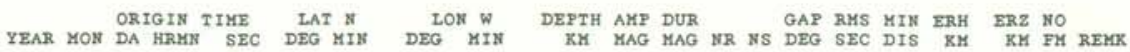

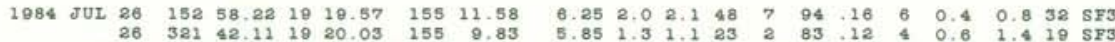

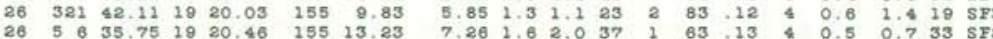

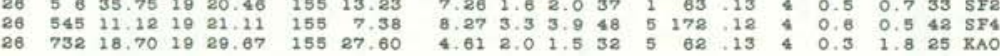

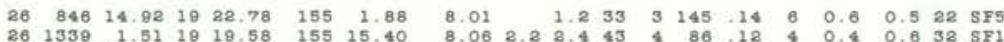

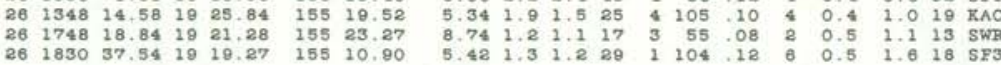

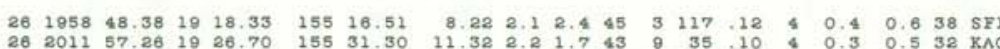

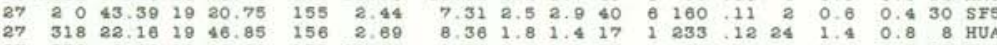

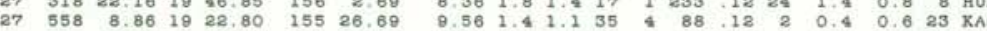

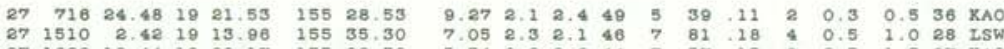

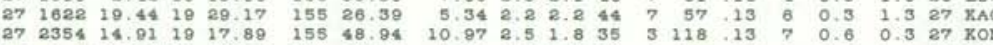

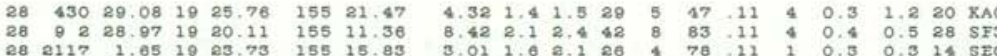

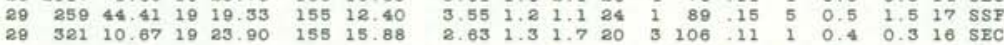

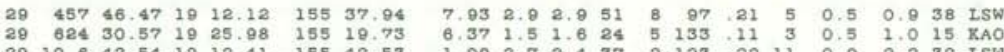

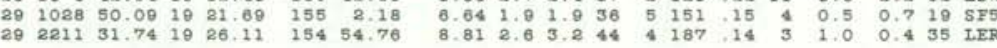
$\begin{array}{rrrrrrrrrrrrrrrrrrrr}30 & 310 & 40.13 & 19 & 21.59 & 155 & 3.03 & 7.16 & 1.6 & 1.3 & 34 & 3 & 122 & .14 & 3 & 0.5 & 0.8 & 28 & \text { SF5 } \\ 30 & 1040 & 59.78 & 19 & 19.53 & 155 & 11.45 & 7.20 & 1.3 & 26 & 0 & 95 & .08 & 5 & 0.5 & 1.1 & 20 & \text { SFF }\end{array}$

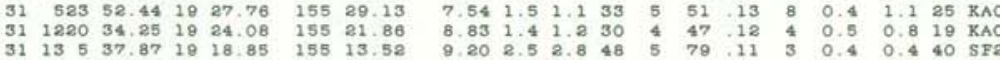

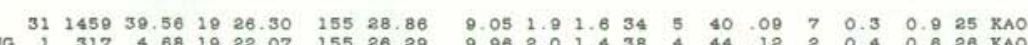

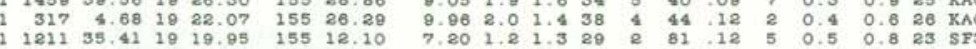

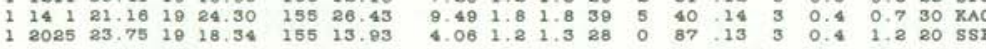

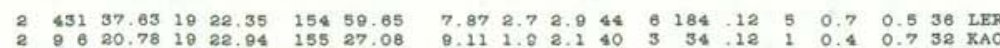

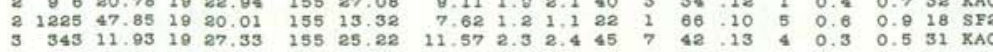

$\operatorname{sing} 34$

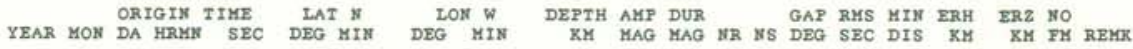
1984 AUG

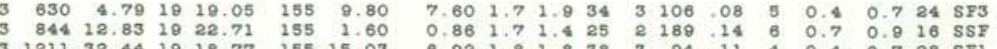

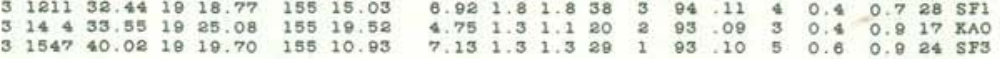

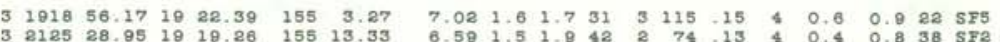

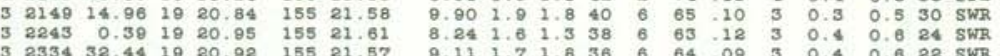

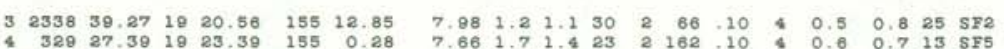

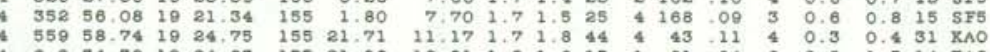

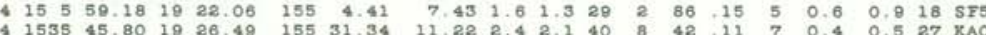

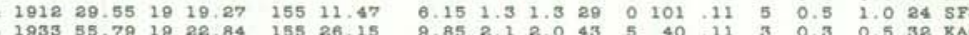

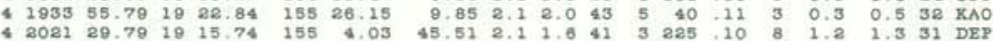

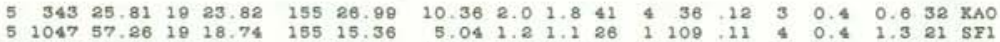

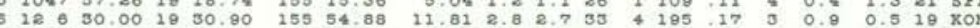
$\begin{array}{rrrrrrrrrrrrrrrrrrrr}5 & 1533 & 12.14 & 19 & 18.43 & 155 & 13.07 & 6.76 & 1.6 & 1.6 & 8 & 0 & 142 & 08 & 3 & 1.0 & 2.5 & 3 & 5 \% 2 \\ 6 & 036 & 15.78 & 19 & 20.86 & 155 & 12.47 & 7.46 & 2.0 & 2.5 & 42 & 2 & 65 & 14 & 3 & 0.5 & 0.6 & 36 & \text { SF2 }\end{array}$

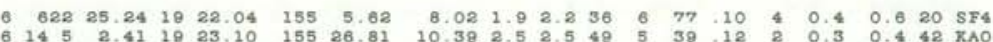

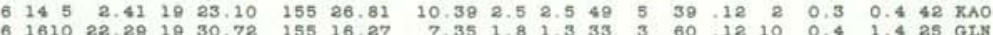

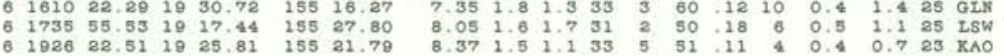

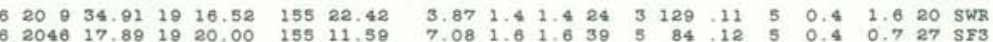

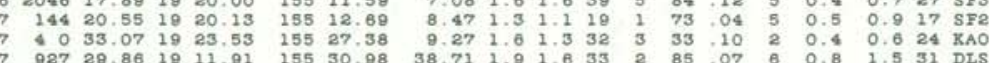
$\begin{array}{lrrrrrrrrrrrrrrrrrr}7 & 941 & 46.55 & 19 & 19.29 & 155 & 15.32 & 8.77 & 2.3 & 2.8 & 44 & 3 & 128 & 12 & 4 & 0.5 & 0.5 & 35 & \text { SF1 } \\ 7 & 1437 & 4.33 & 19 & 42.40 & 155 & 1.48 & 0.05 & 2.4 & 3.1 & 27 & 0 & 203 & 15 & 1 & 1.3 & 0.8 & 14 & \text { HIL B }\end{array}$

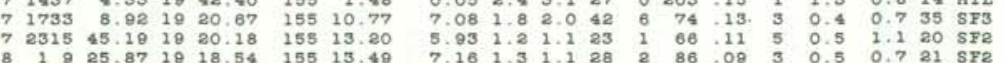
$\begin{array}{llllllllllllllllllll}8 & 332 & 17.13 & 19 & 20.23 & 155 & 11.67 & 7.86 & 1.9 & 2.0 & 44 & 7 & 79 & 13 & 5 & 0.4 & 0.6 & 31 & \text { SF3 } \\ 8 & 711 & 37.32 & 10 & 23.20 & 155 & 1.82 & 8.16 & 1.0 & 2.0 & 39 & 4 & 141 & 15 & 5 & 0.6 & 0.5 & 26 & \text { SF }\end{array}$

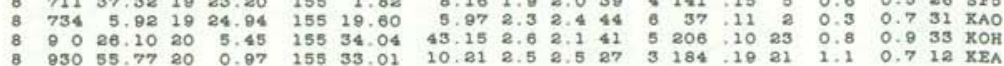




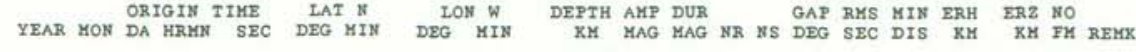

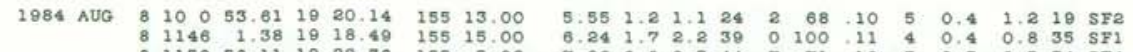

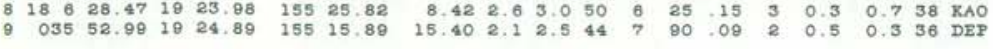

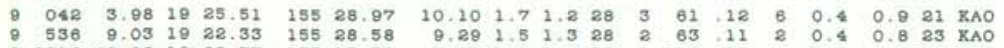

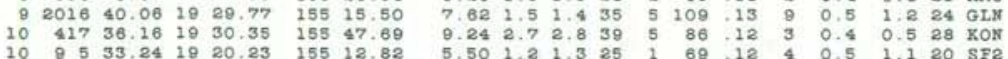

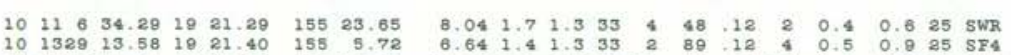

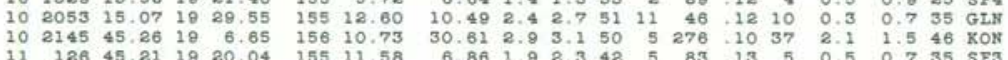

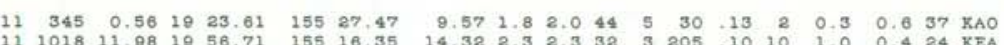

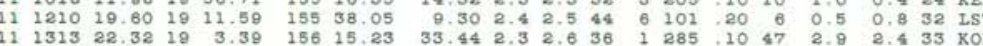

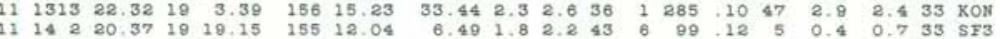

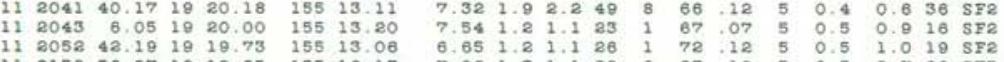

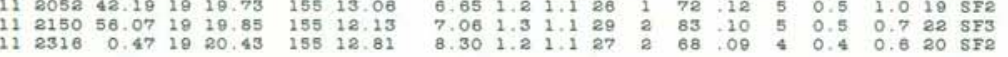

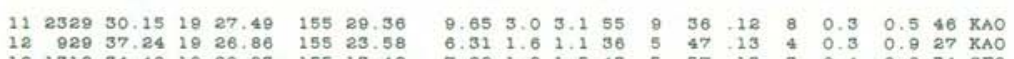

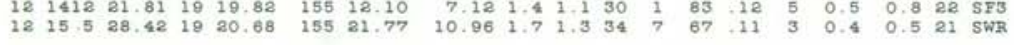
$\begin{array}{llllllllllllllllllll}12 & 1513 & 49.98 & 19 & 15.28 & 155 & 22.47 & 6.35 & 1.2 & 1.5 & 23 & 2 & 175 & 10 & 3 & 0.6 & 1.0 & 15 & \text { SWR } \\ 12 & 1611 & 7.48 & 18 & 19.12 & 155 & 10.98 & 6.22 & 1.3 & 1.2 & 34 & 3 & 108 & 14 & 6 & 0.5 & 0.8 & 28 & \text { SF3 }\end{array}$

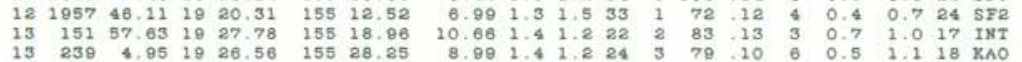

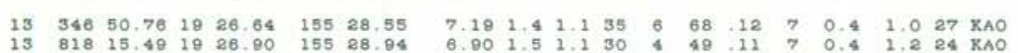

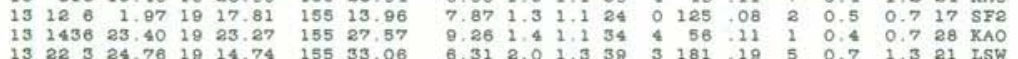

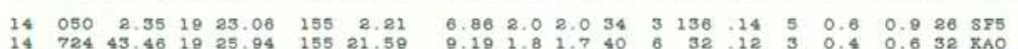

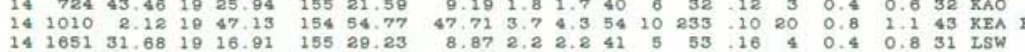

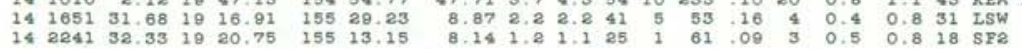

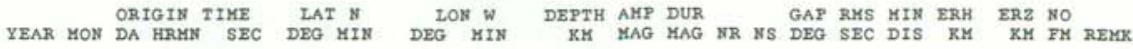

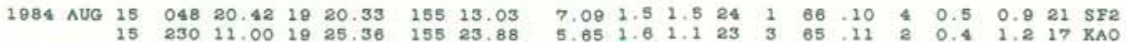

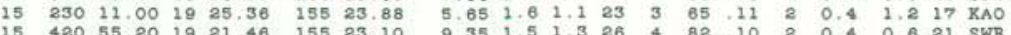

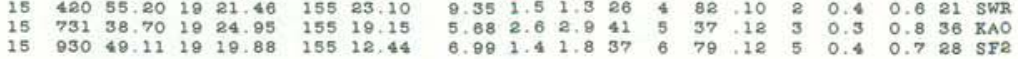

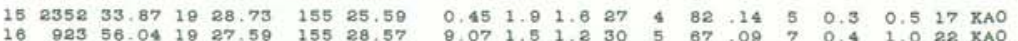

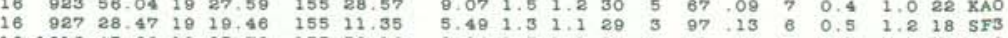

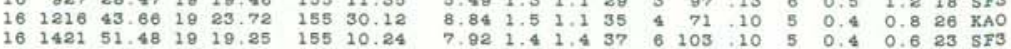

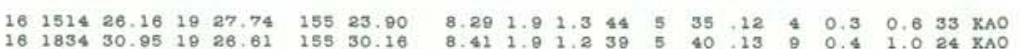

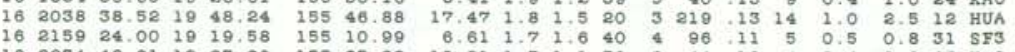

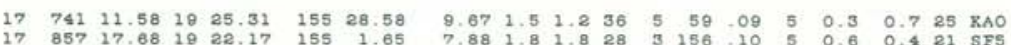

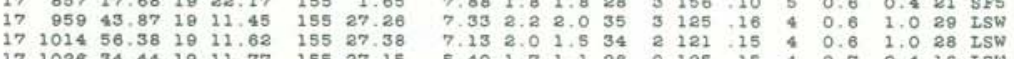

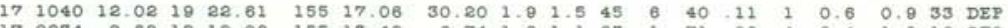

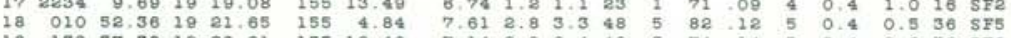

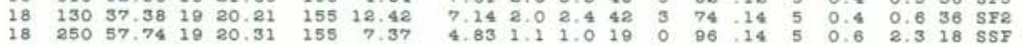
$\begin{array}{llllllllllllllllllll}18 & 4 & 49.02 & 19 & 5.40 & 156 & 11.65 & 34.18 & 2.6 & 2.3 & 42 & 3 & 278 & .09 & 39 & 2.0 & 1.4 & 36 & \text { XON } \\ 18 & 648 & 42.46 & 19 & 20.98 & 156 & 14.96 & 33.26 & 2.1 & 1.7 & 20 & 1 & 286 & 11 & 38 & 3.8 & 2.4 & 18 & \text { Kos }\end{array}$

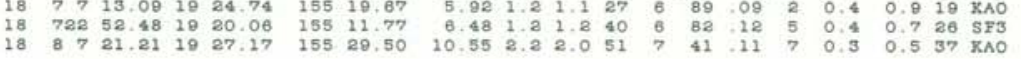
$\begin{array}{lllllllllllllllllll}18 & 855 & 13.64 & 19 & 21.68 & 155 & 26.45 & 10.26 & 2.3 & 2.5 & 46 & 5 & 46 & 12 & 3 & 0.4 & 0.5 & 37 & \text { XAO } \\ 18 & 952 & 18.20 & 10 & 19.49 & 155 & 30.86 & 7.69 & 1.6 & 1.2 & 33 & 3 & 61 & 12 & 8 & 0.3 & 1.0 & 23 & \text { XAO }\end{array}$

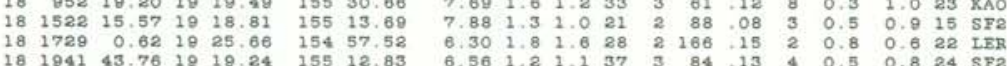

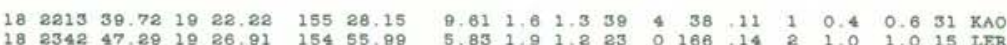

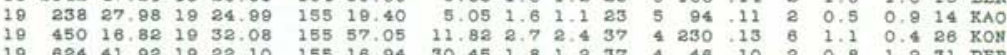
$\begin{array}{lllllllllllllllllll}19 & 7 & 75.91 & 19 & 16.88 & 155 & 23.49 & 42.98 & 2.0 & 1.6 & 48 & 8 & 104 & .12 & 5 & 0.7 & 1.1 & 38 & \text { DEP }\end{array}$

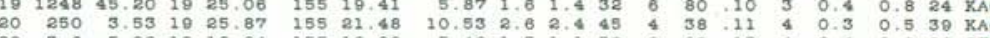

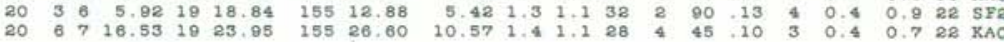


1984 HVO EARTHQUAXE SUMHARY LIST

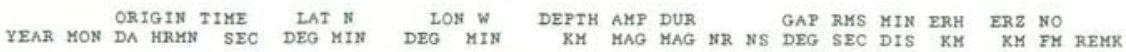

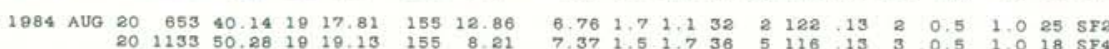

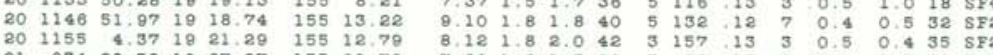
$\begin{array}{rrrrrrrrrrrrrrrrrrrrr}21 & 253 & 32.59 & 19 & 19.28 & 155 & 15.56 & 5.07 & 1.1 & 1.3 & 23 & 2 & 101 & 12 & 6 & 0.4 & 1.8 & 21 & \text { SF } \\ 21 & 5 & 3 & 40.80 & 19 & 12.64 & 155 & 5.89 & 49.54 & 2.3 & 2.1 & 34 & 1 & 237 & 09 & 9 & 2.0 & 2.3 & 32 & \text { DEF }\end{array}$

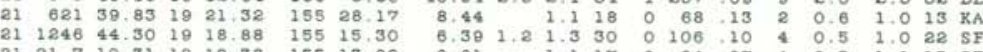

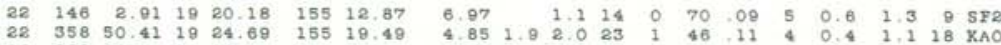

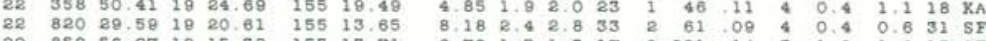

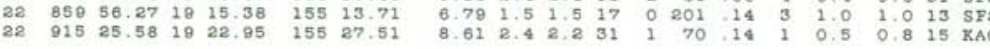

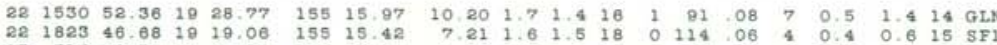

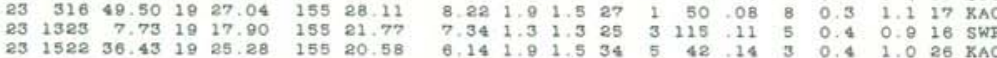

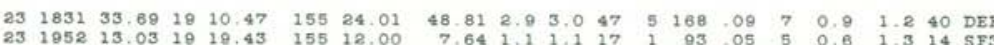

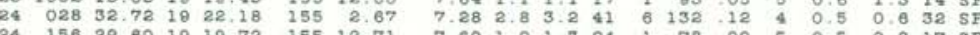

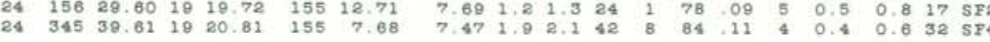
$\begin{array}{rrrrrrrrrrrrrrrrrrr}24 & 634 & 41.83 & 19 & 17.69 & 155 & 12.89 & 5.26 & 1.3 & 1.3 & 20 & 3 & 122 & 07 & 1 & 0.5 & 0.9 & 19 & \mathrm{sF2} \\ 24 & 1019 & 19.00 & 19 & 20.18 & 155 & 24.42 & 13.25 & 1.9 & 1.8 & 36 & 4 & 65 & 09 & 2 & 0.4 & 0.3 & 27 & \text { DEF }\end{array}$

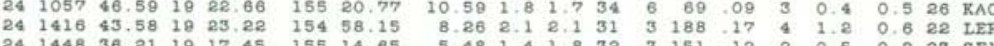

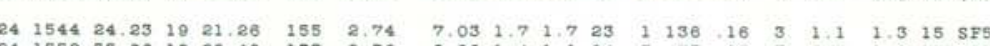

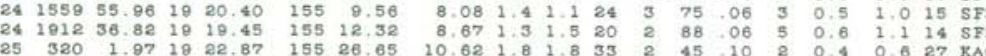

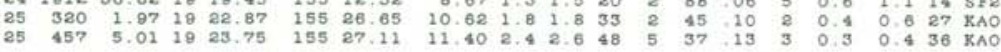
$\begin{array}{rrrrrrrrrrrrrrrrrrrrr}25 & 946 & 37.37 & 19 & 5.27 & 155 & 19.75 & 23.59 & 2.7 & 2.7 & 39 & 4 & 243 & 09 & 18 & 1.2 & 1.7 & 35 & \text { LOI } \\ 25 & 1120 & 1.92 & 19 & 20.11 & 155 & 24.55 & 13.00 & 2.7 & 2.8 & 49 & 8 & 65 & 11 & 2 & 0.4 & 0.3 & 40 & \text { SWR }\end{array}$

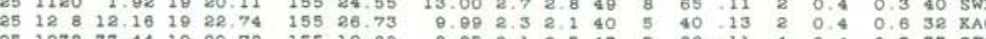

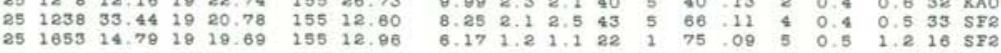

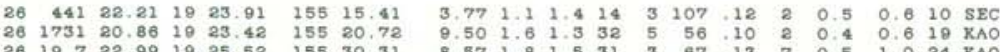

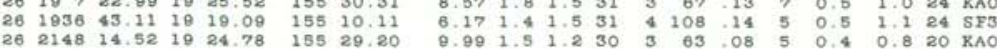

1984 HVO EARTHQUARE SUMMARY LIST

PAGE 38

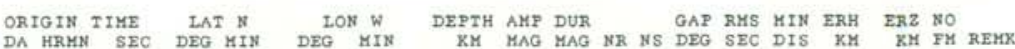
1984 AUG $27 \quad \begin{array}{rlllllllllllllllllllll}558 & 37.89 & 19 & 17.86 & 155 & 21.19 & 5.61 & 1.3 & 1.1 & 12 & 1 & 122 & 10 & 5 & 0.6 & 1.6 & 7 & \text { SWR }\end{array}$

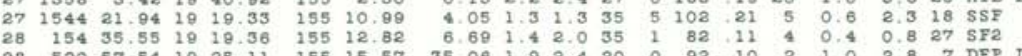

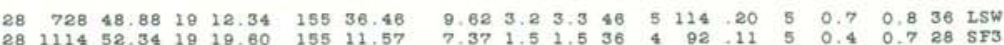

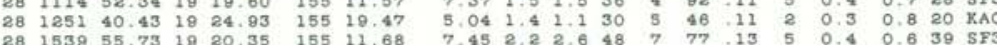

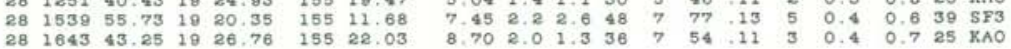
$\begin{array}{llllllllllllllllllll}28 & 2115 & 0.35 & 19 & 23.90 & 155 & 30.90 & 8.80 & 1.8 & 1.2 & 34 & 2 & 49 & 10 & 6 & 0.4 & 0.8 & 27 & \text { KAO } \\ 28 & 2230 & 14.33 & 19 & 23.41 & 155 & 20.91 & 9.72 & 1.6 & 2.0 & 38 & 5 & 55 & 12 & 2 & 0.4 & 0.5 & 27 & \text { KAO }\end{array}$

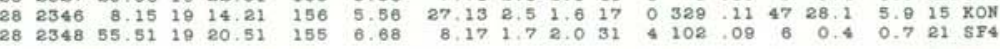

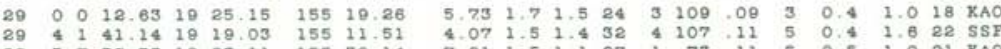

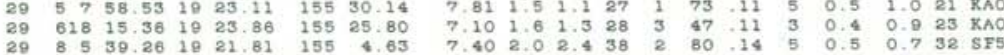

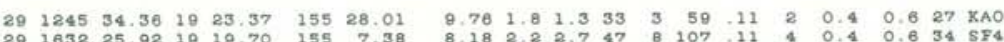

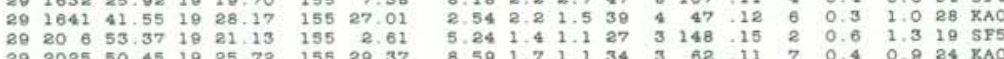
$\begin{array}{lrlllllllllllllllll}29 & 2324 & 22.35 & 19 & 20.50 & 155 & 12.91 & 7.19 & 1.4 & 1.5 & 34 & 3 & 66 & 15 & 4 & 0.5 & 0.8 & 23 & \mathbf{5 F 2} \\ 30 & 142 & 46.78 & 19 & 23.75 & 155 & 27.19 & 9.73 & 1.8 & 1.8 & 40 & 4 & 62 & 12 & 2 & 0.4 & 0.6 & 32 & \text { KAO }\end{array}$

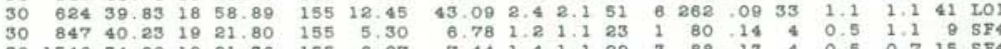

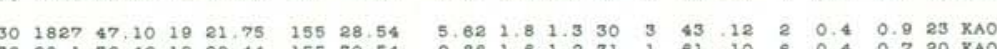

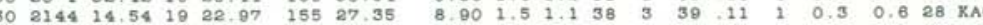

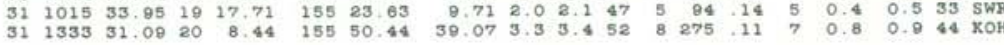

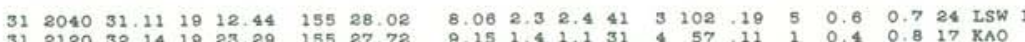

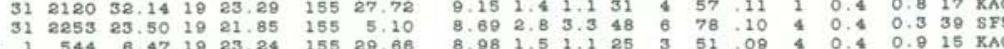

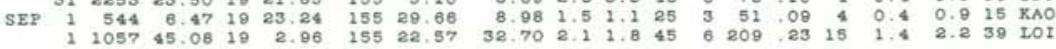

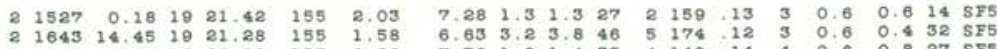

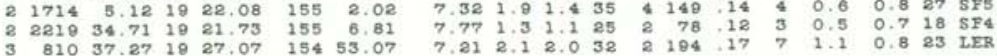




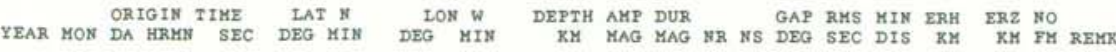

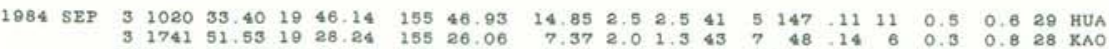

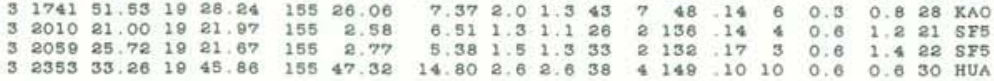

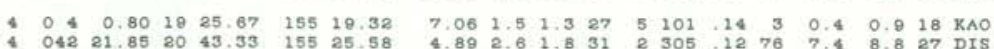

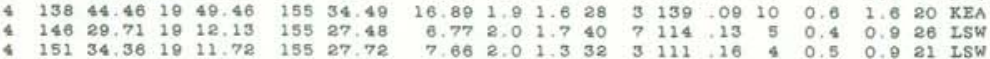

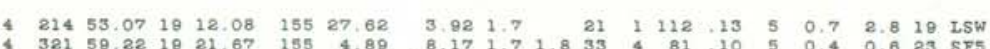

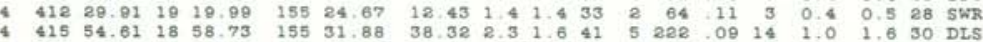

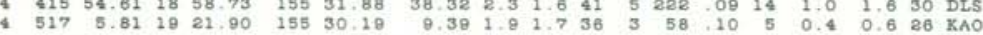

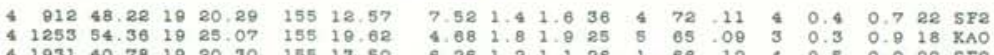

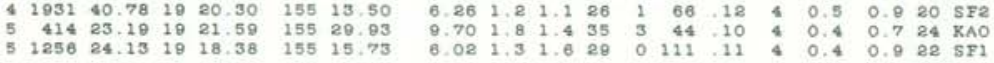

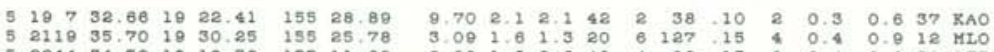

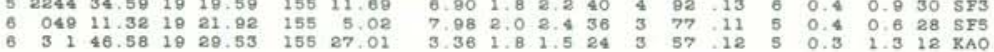

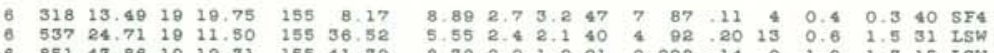

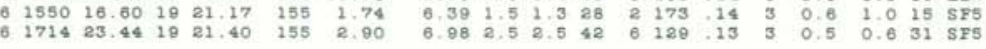

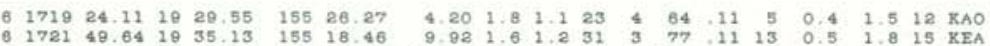

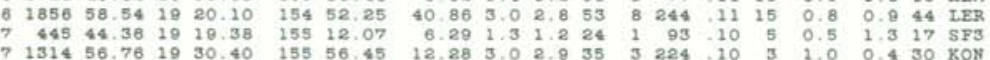

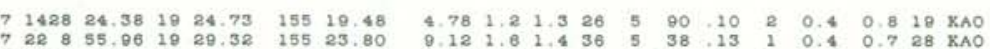

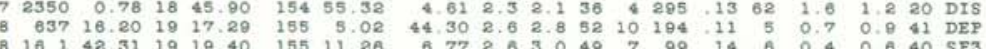

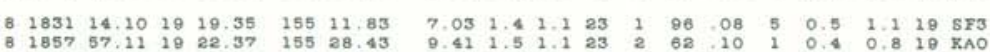

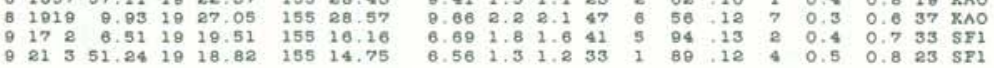

1984 HVO EARTHQUARE SUMMARY LIST

PAGE 40

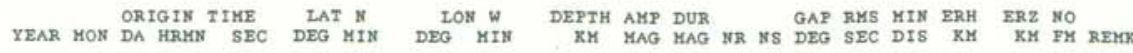

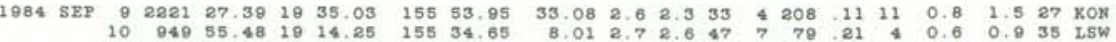

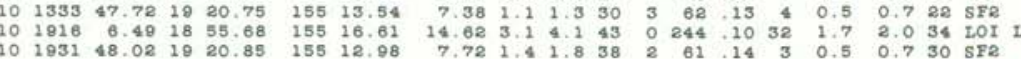

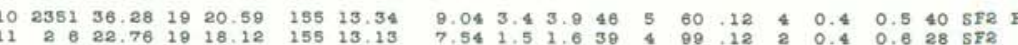

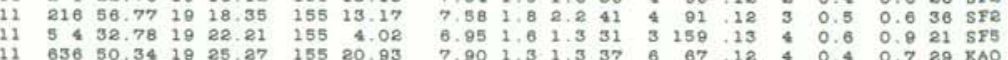

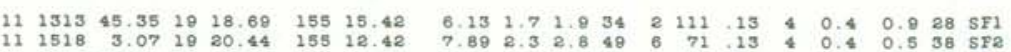

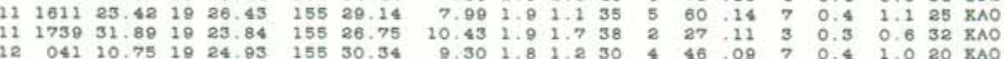

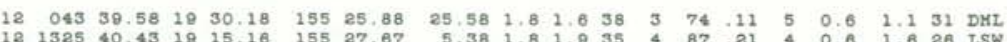

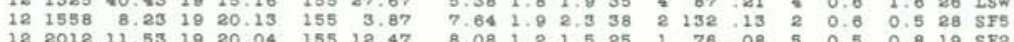

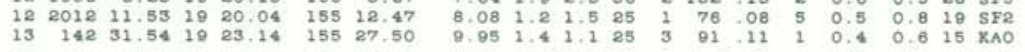

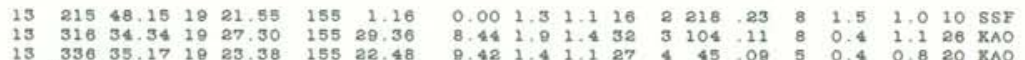

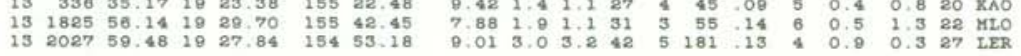

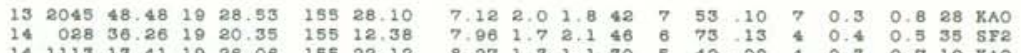

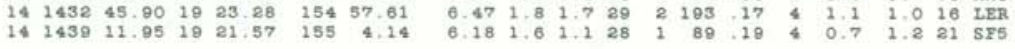

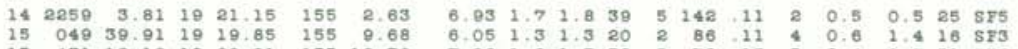

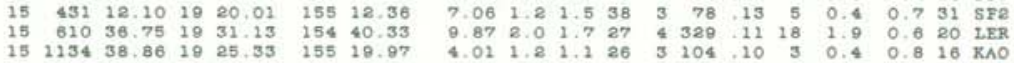

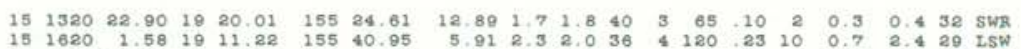

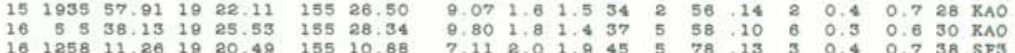

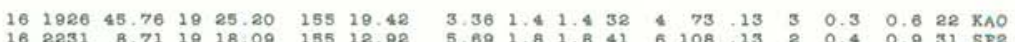

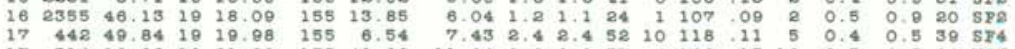




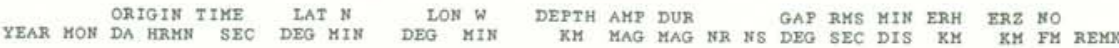

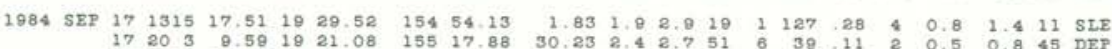

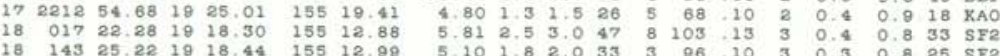

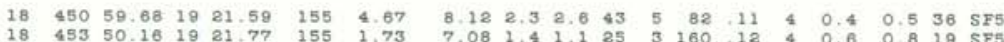

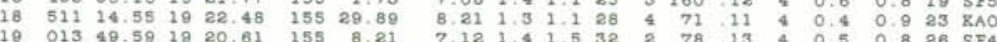

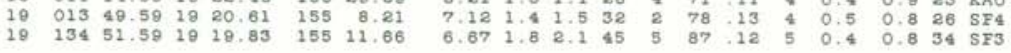
$\begin{array}{lllllllllllllllllll}19 & 630 & 15.24 & 18 & 26.65 & 155 & 30.21 & 9.02 & 1.9 & 1.5 & 40 & 5 & 44 & 12 & 9 & 0.4 & 0.8 & 29 & \text { KAO } \\ 19 & 715 & 18.14 & 19 & 20.06 & 155 & 12.51 & 7.55 & 2.00 & 2.5 & 45 & 4 & 63 & 13 & 3 & 0.4 & 0.6 & 37 & \text { SF2 }\end{array}$

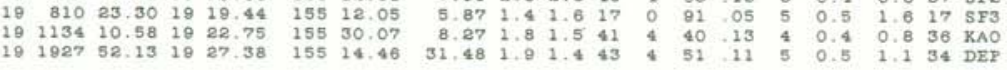

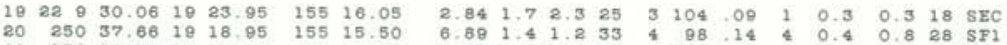

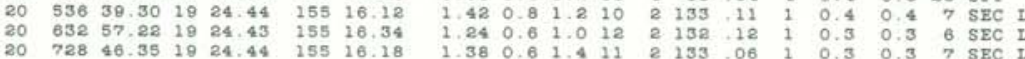

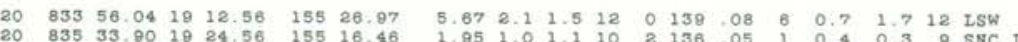

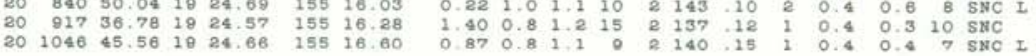

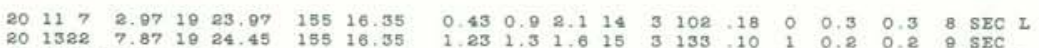

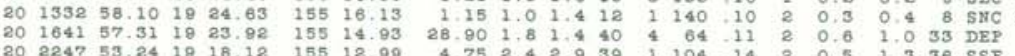

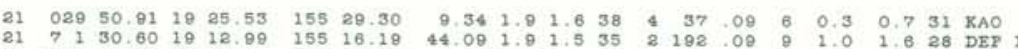

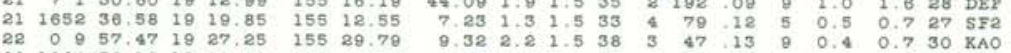

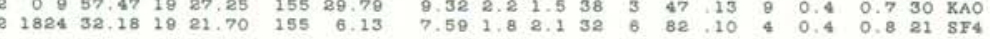

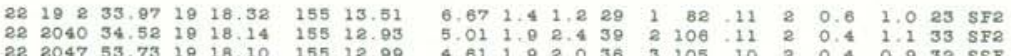

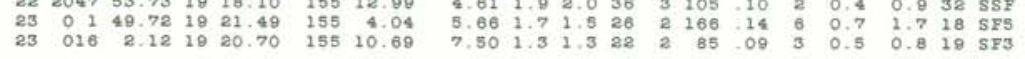

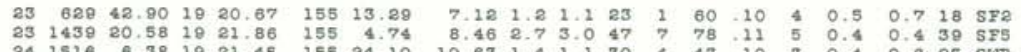

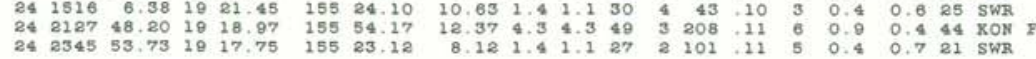

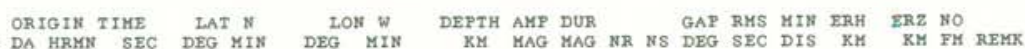

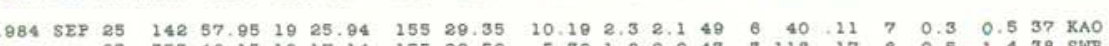

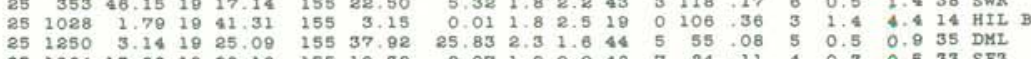

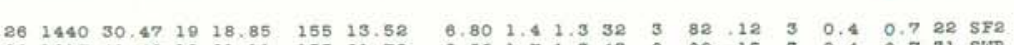

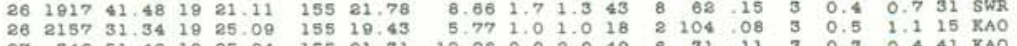

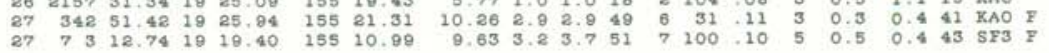

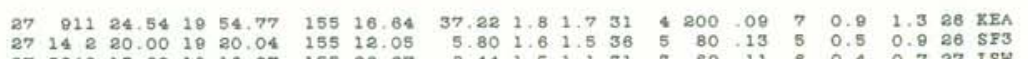

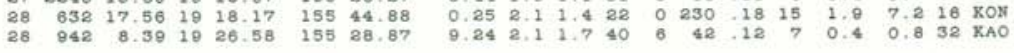

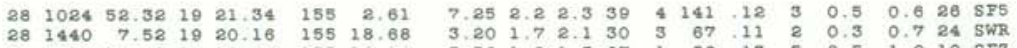

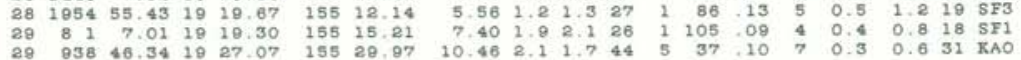

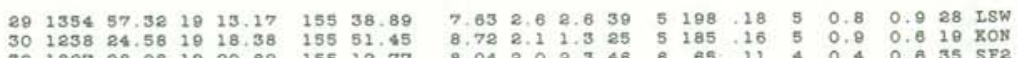

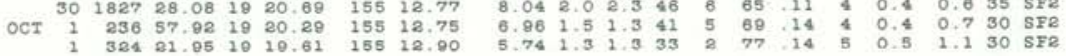

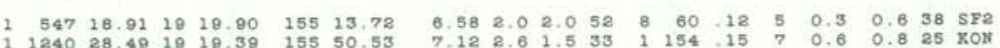

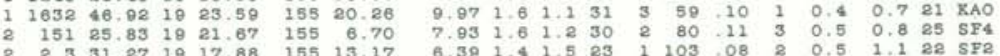

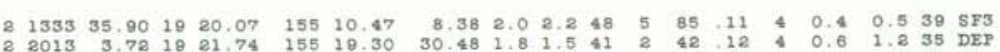

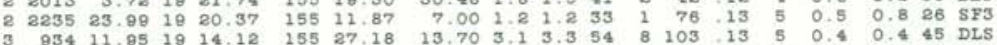

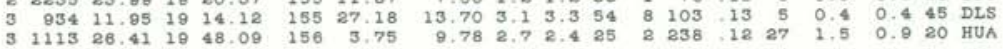

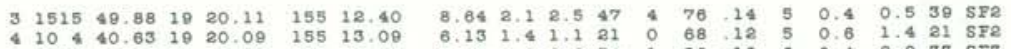

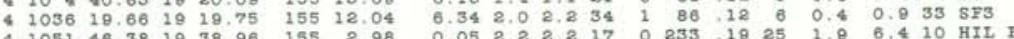

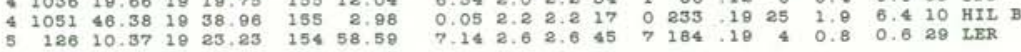

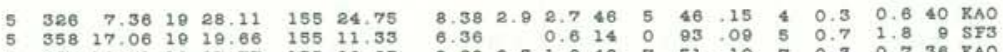

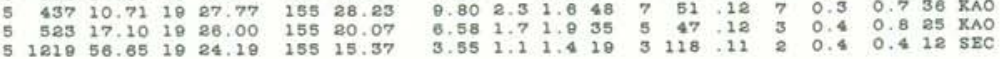




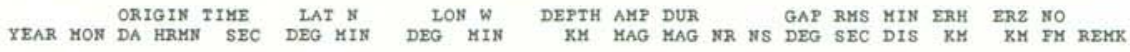

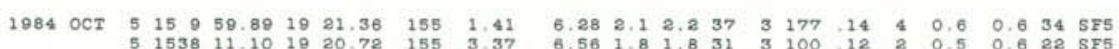

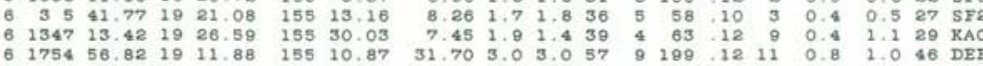

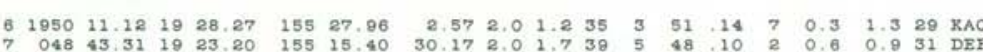

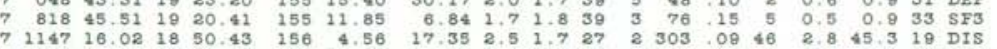

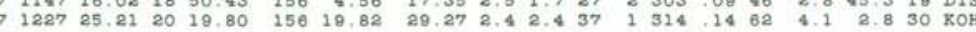
$\begin{array}{llllllllllllllllllll}7 & 2112 & 55.32 & 19 & 25.35 & 155 & 19.65 & 6.32 & 1.4 & 1.2 & 30 & 5 & 89 & 12 & 3 & 0.4 & 0.9 & 24 & \text { XAO } \\ 7 & 2114 & 53.72 & 19 & 25.28 & 155 & 10.70 & 6.32 & 1.3 & 1.1 & 24 & 4 & 87 & 10 & 3 & 0.4 & 0.9 & 17 & \text { XAO }\end{array}$

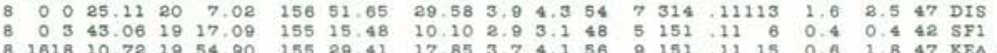

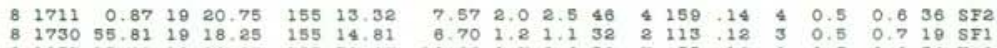

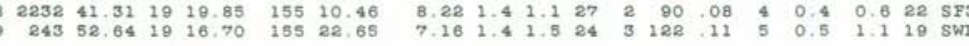

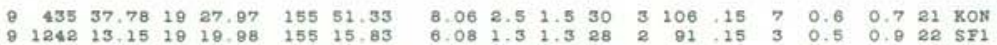

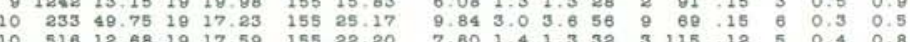

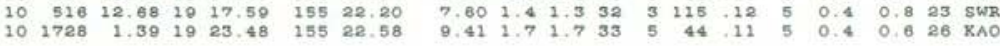

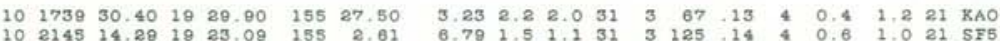

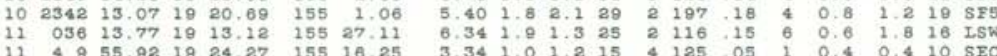

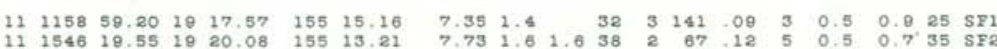

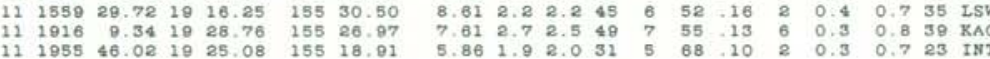

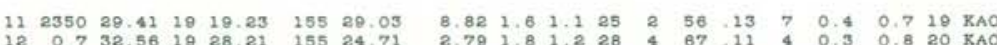

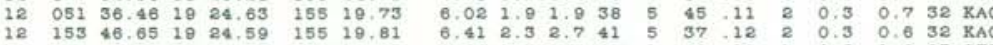

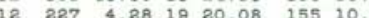

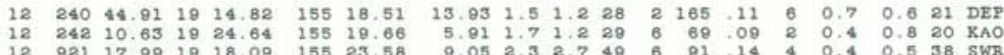

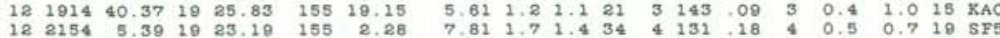

1984 HVO EARTHQUAKE SUMMARY LIST

PAGE 44

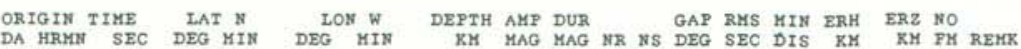

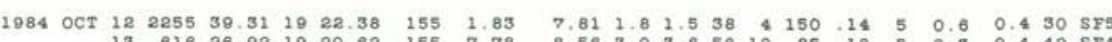

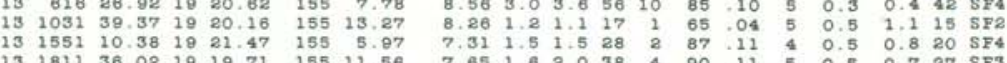

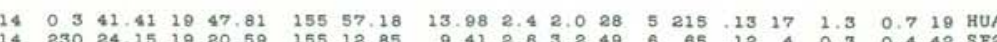

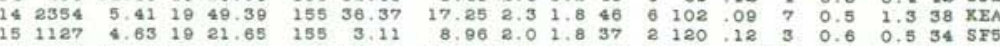

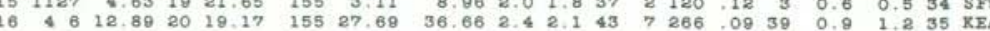

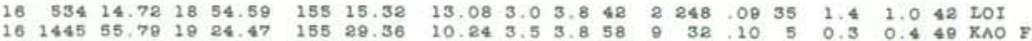

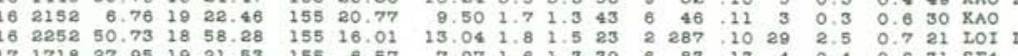

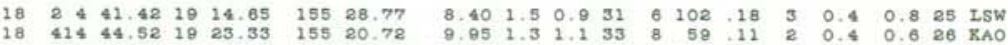

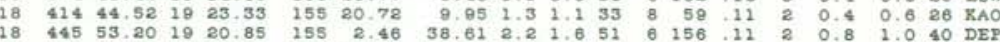

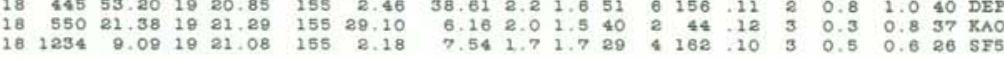

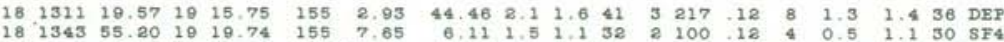

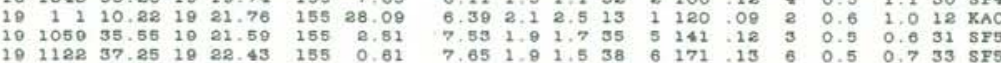

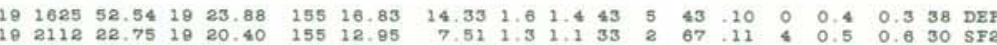
19813647.43

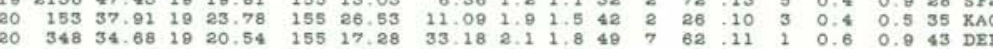

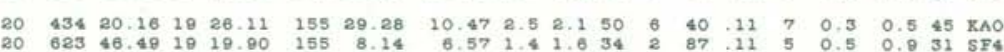

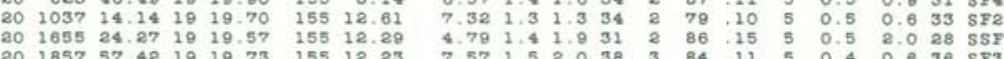

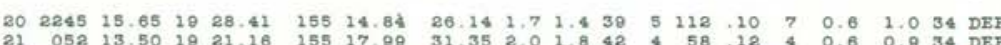

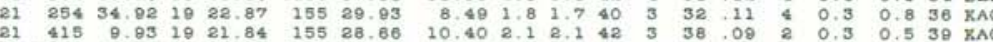

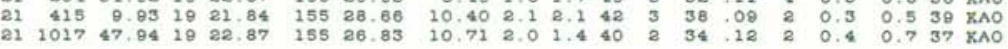

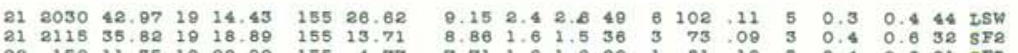

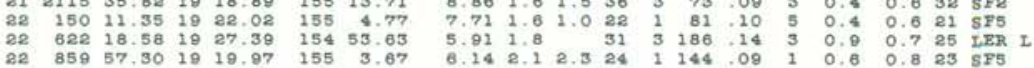




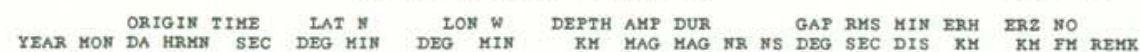

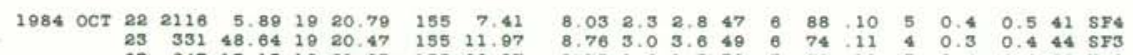

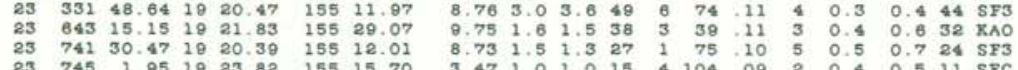

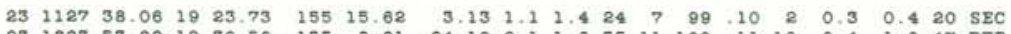

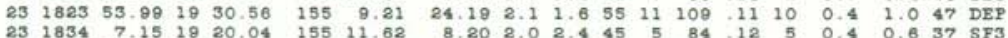

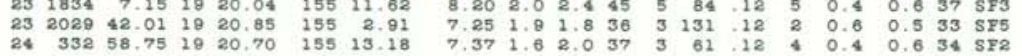

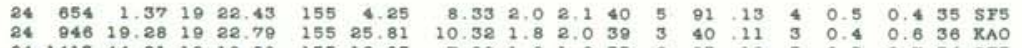

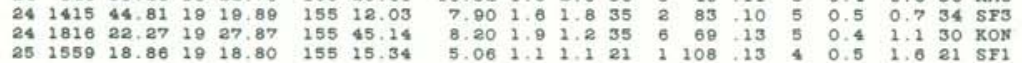

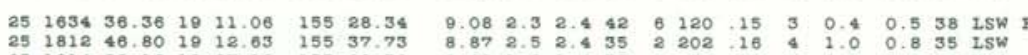

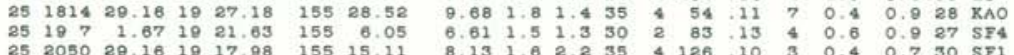

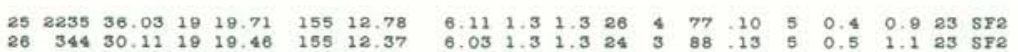

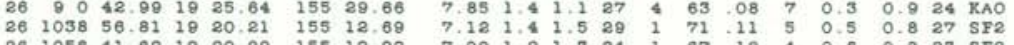

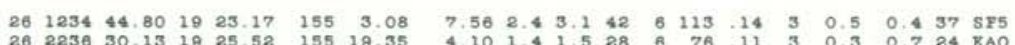

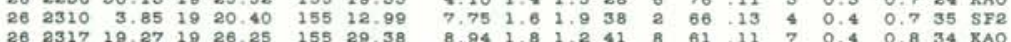

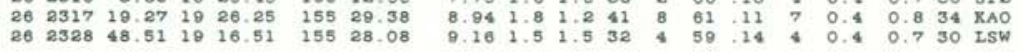
$\begin{array}{llllllllllllllllllll}26 & 2330 & 54.58 & 19 & 25.39 & 155 & 19.42 & 5.35 & 1.3 & 1.3 & 26 & 7 & 117 & 10 & 3 & 0.4 & 0.8 & 21 & \text { XAO } \\ 27 & 012 & 28.21 & 10 & 25.24 & 155 & 19.34 & 5.93 & 2.0 & 1.9 & 28 & 5 & 46 & 11 & 3 & 0.4 & 0.7 & 24 & \text { KAO }\end{array}$

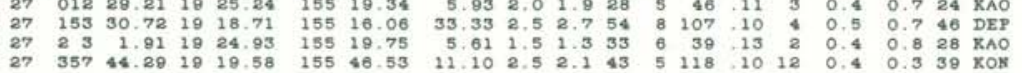

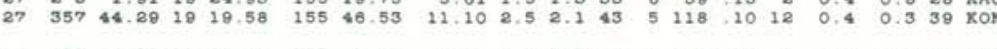

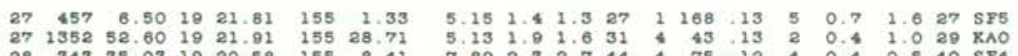

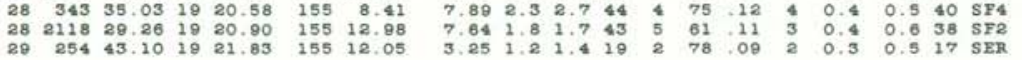

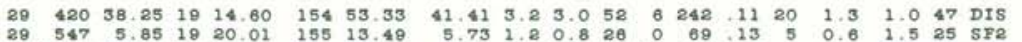

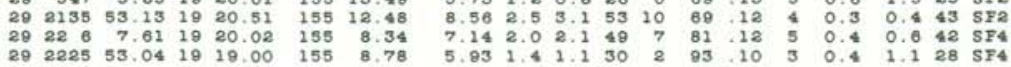

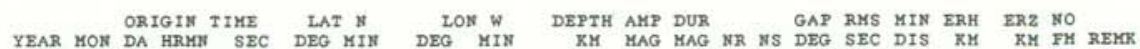
$\begin{array}{rlrlllllllllllllllll}1984 & \text { OCT } 29 & 2311 & 9.99 & 19 & 20.62 & 155 & 12.00 & 9.27 & 2.7 & 3.2 & 51 & 6 & 72 & 12 & 4 & 0.3 & 0.4 & 44 & \text { SF3 } \\ 30 & 213 & 42.84 & 10 & 21.43 & 155 & 30.19 & 9.06 & 2.2 & 2.2 & 41 & 3 & 47 & 10 & 5 & 0.3 & 0.7 & 38 & \text { XAO }\end{array}$

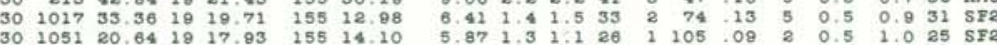

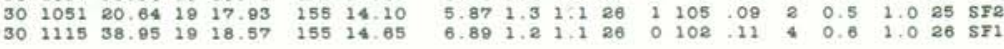

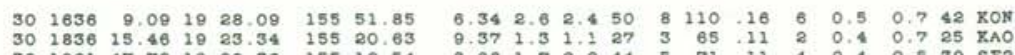

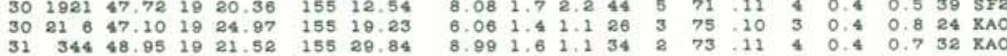

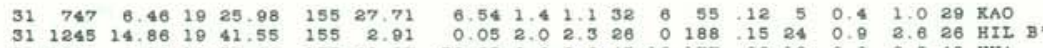

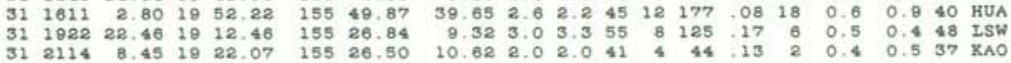

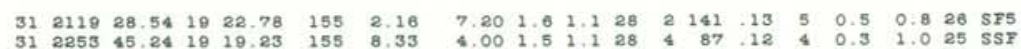
sov

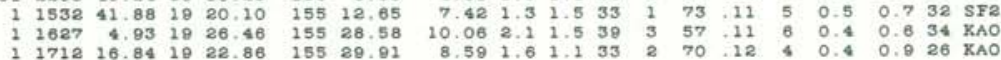

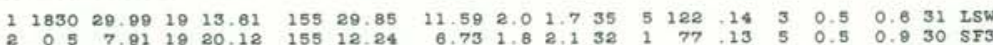

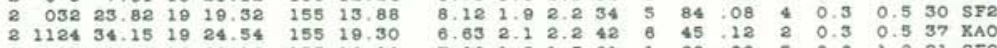

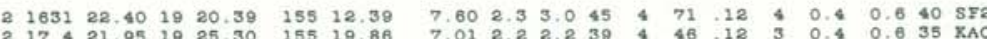

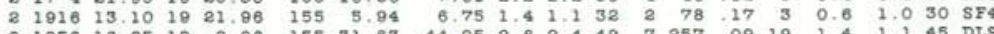

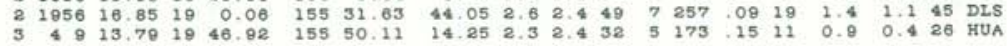
$\begin{array}{lllllllllllllllllllll}3 & 545 & 13.54 & 19 & 25.40 & 155 & 27.91 & 9.67 & 1.9 & 1.5 & 38 & 5 & 56 & 12 & 5 & 0.4 & 0.8 & 31 & \text { KAO } \\ 3 & 620 & 14.68 & 19 & 19.82 & 155 & 10.04 & 6.32 & 1.3 & 1.1 & 20 & 1 & 89 & 10 & 4 & 0.8 & 1.3 & 19 & \text { SF3 } \\ 3\end{array}$

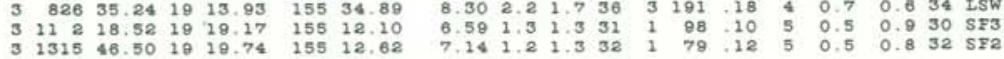

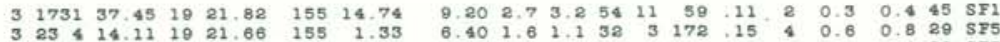

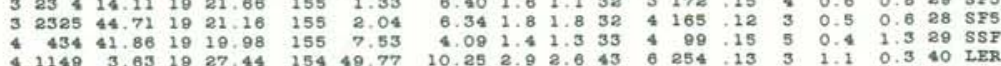

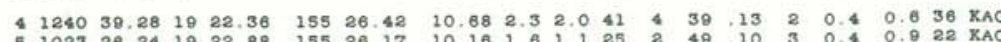

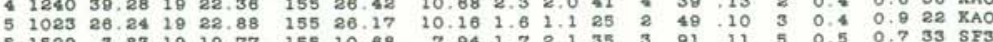

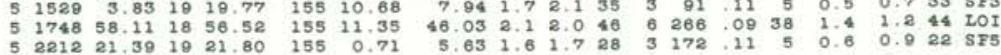


1984 HVO EARTHQUAKE SUMKARY LIST

PAGE 47

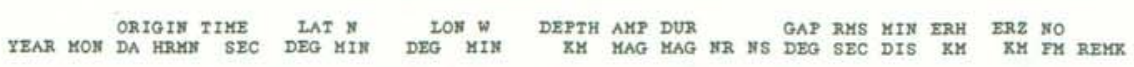

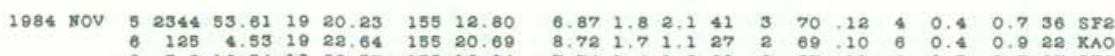

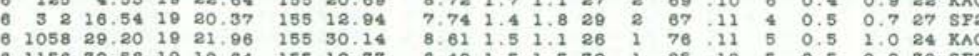

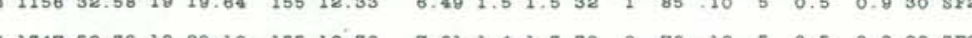

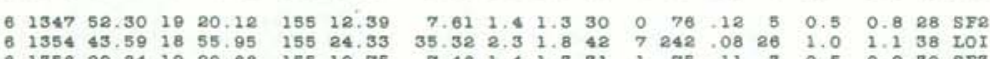

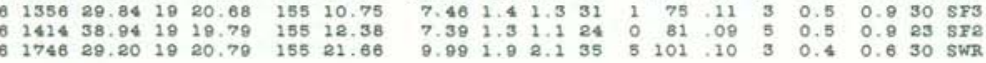
$\begin{array}{rrrrrrrrrrrrrrrrrrr}7 & 021 & 7.78 & 19 & 14.53 & 195 & 34.83 & 7.64 & 2.2 & 1.5 & 32 & 6 & 78 & 18 & 4 & 0.5 & 0.8 & 28 & \text { LSW } \\ 7 & 149 & 37.51 & 19 & 19.49 & 155 & 10.52 & 6.71 & 1.3 & 1.3 & 19 & 1 & 218 & 11 & 5 & 1.4 & 1.5 & 18 & \text { SF3 }\end{array}$

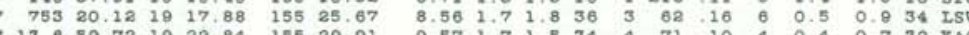

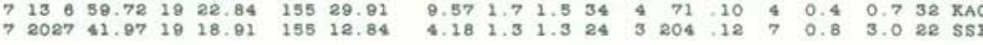

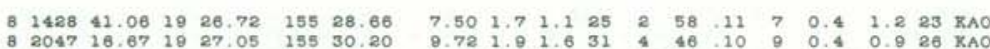

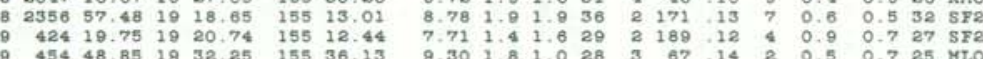

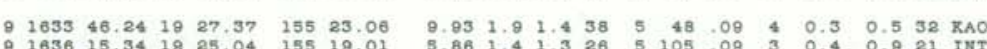

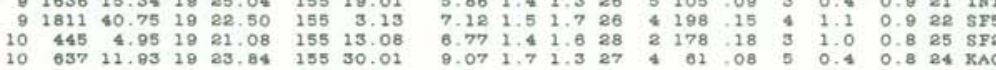

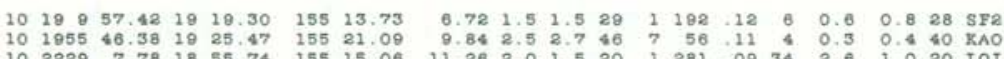

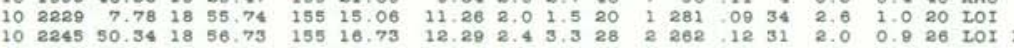

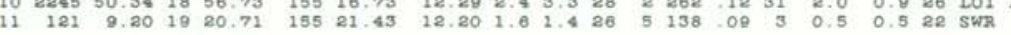

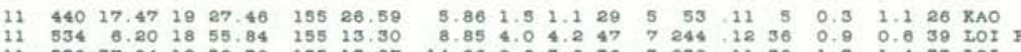

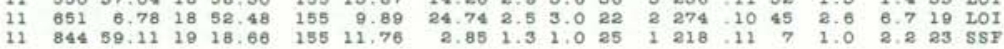

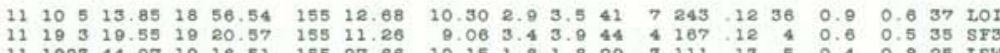

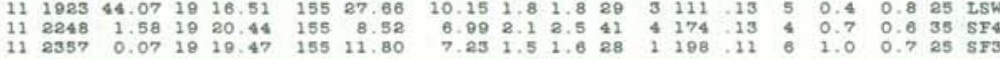

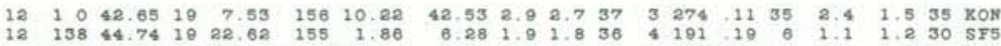

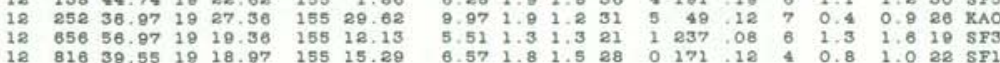

1984 HVO EARTHQUARE SUMMARY LIST

PAGE 48 ORTGIN TIME LAT

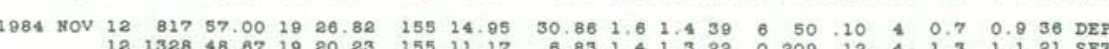

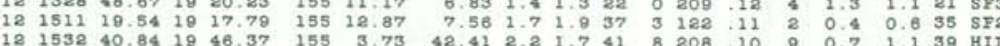

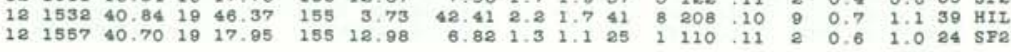

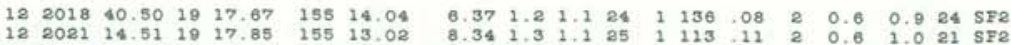

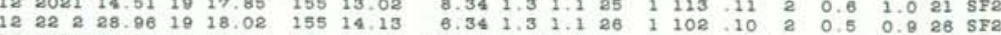

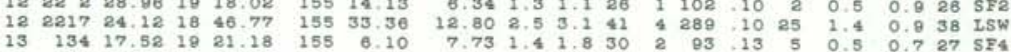

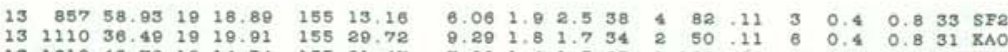

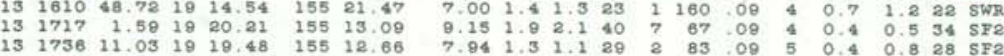

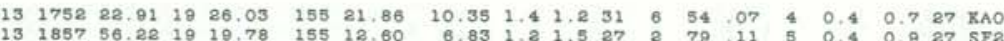

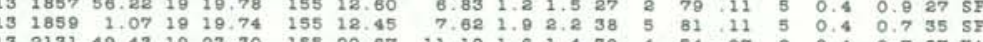

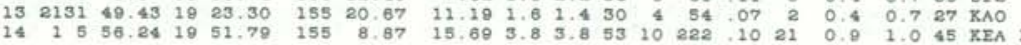

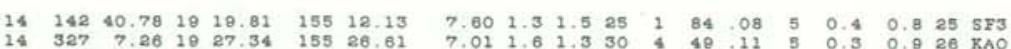

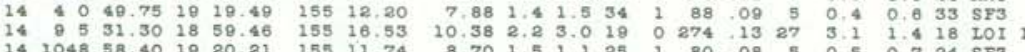

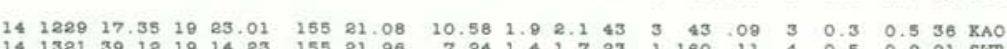

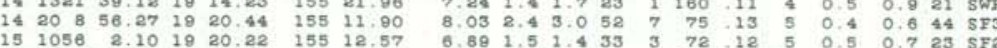

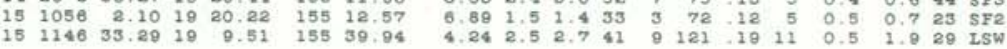

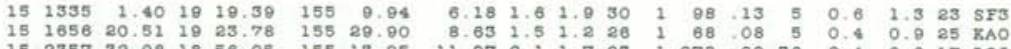
$\begin{array}{lllllllllllllllllll}15 & 2357 & 32.08 & 18 & 56.05 & 155 & 13.05 & 11.97 & 2.1 & 1.7 & 23 & 1 & 278 & 00 & 36 & 2.4 & 0.8 & 17 & 107\end{array}$

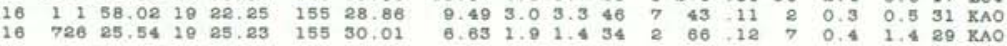

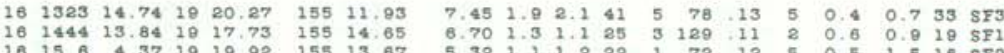

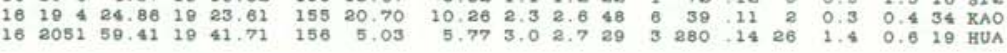

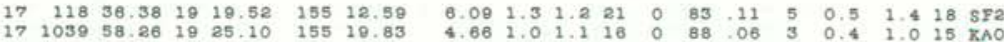

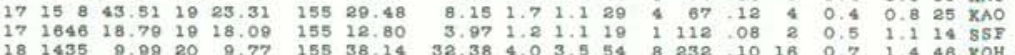




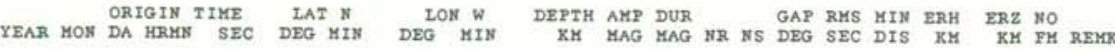

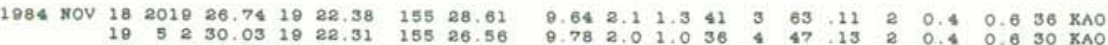

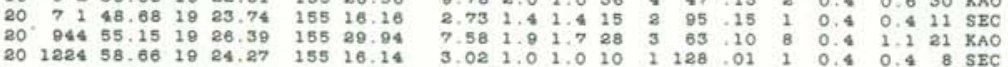

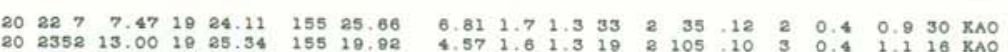

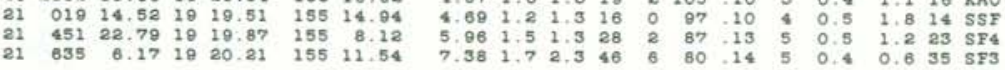

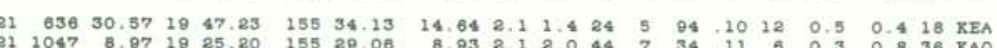

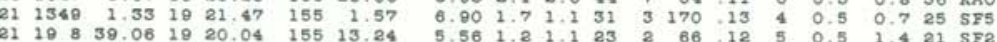

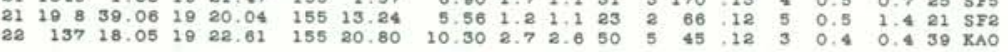
$\begin{array}{lllllllllllllllllll}22 & 533 & 57.73 & 19 & 19.89 & 155 & 7.79 & 5.29 & 1.4 & 1.1 & 28 & 1 & 95 & 13 & 5 & 0.5 & 1.5 & 21 & 574 \\ 22 & 748 & 52.37 & 10 & 20.39 & 159 & 11.52 & 7.33 & 1.9 & 2.6 & 24 & 1 & 77 & 10 & 4 & 0.5 & 1.1 & 14 & 5 F 3\end{array}$

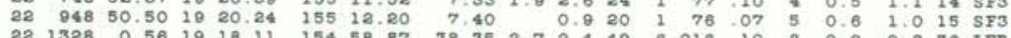

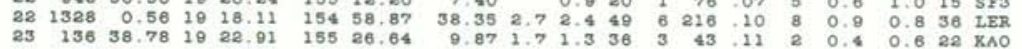

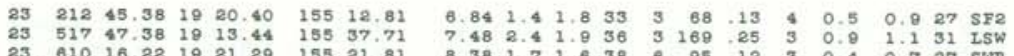

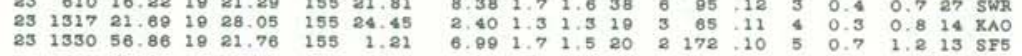
$\begin{array}{lllllllllllllllllllll}23 & 1426 & 46.43 & 19 & 20.80 & 155 & 12.41 & 7.09 & 1.6 & 1.5 & 31 & 2 & 69 & 12 & 4 & 0.5 & 0.7 & 23 & 572 \\ 23 & 2059 & 15 & 18 & 19 & 25.73 & 155 & 29.12 & 10.24 & 1.6 & 1.4 & 33 & 4 & 37 & 12 & 7 & 0.4 & 0.7 & 21 & \text { KAO }\end{array}$

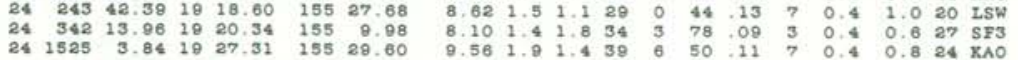

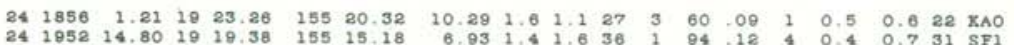

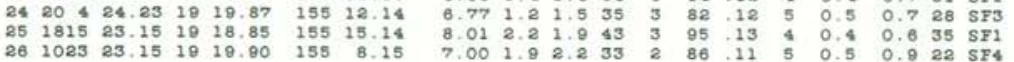

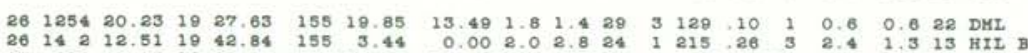

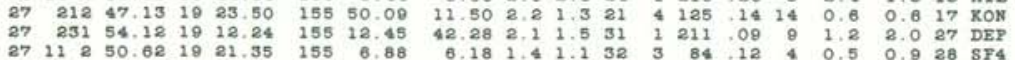

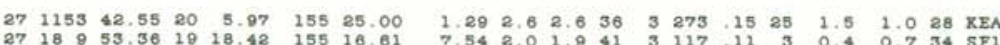

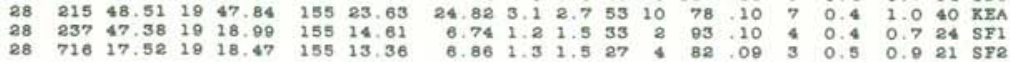

1984 HVO EARTHQUAKE SUMHARY LIST

PAGE 50

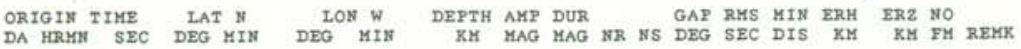

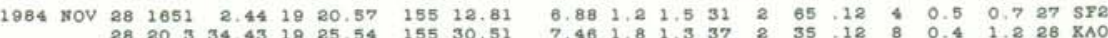

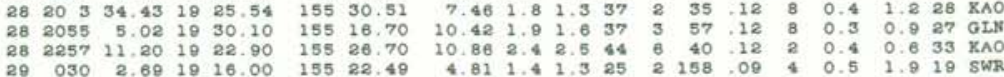

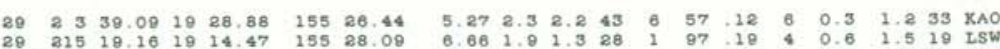

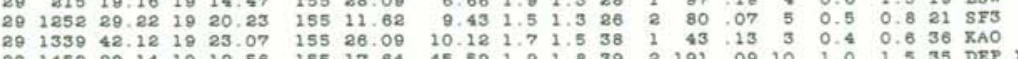

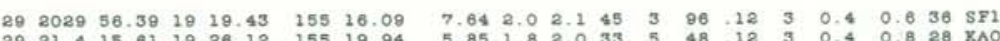

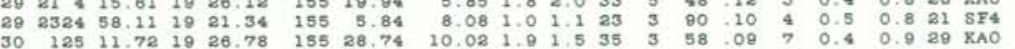

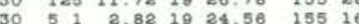

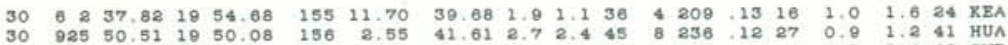

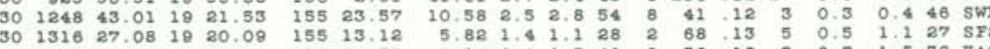

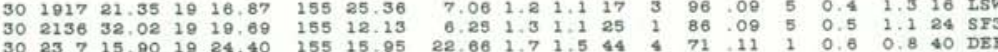

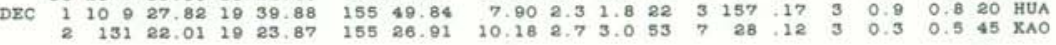
$\begin{array}{llllllllllllllllllll}2 & 651 & 23.04 & 19 & 19.25 & 155 & 11.49 & 7.38 & 1.4 & 1.5 & 27 & 1 & 102 & 08 & 5 & 0.5 & 1.0 & 25 & \text { SF3 } \\ 2 & 652 & 45.55 & 19 & 25.86 & 155 & 28.85 & 9.34 & 1.8 & 1.4 & 31 & 3 & 60 & 10 & 6 & 0.4 & 1.0 & 23 & \text { KAO }\end{array}$

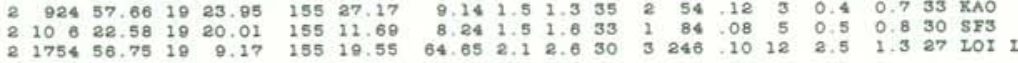

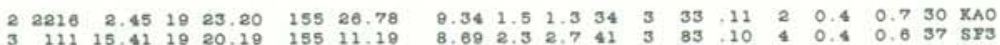

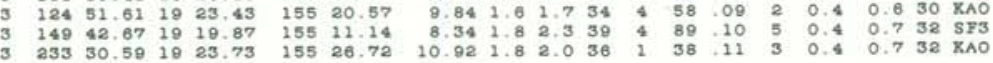

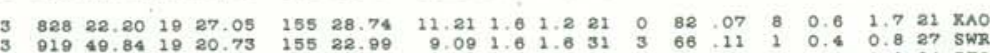

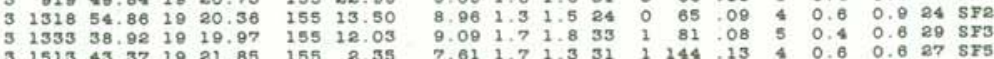

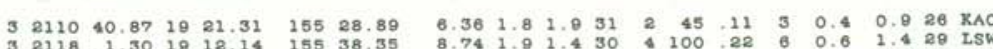

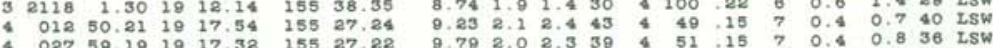

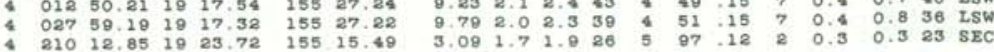




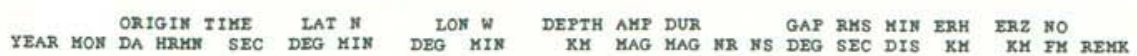

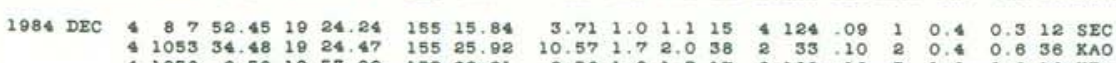

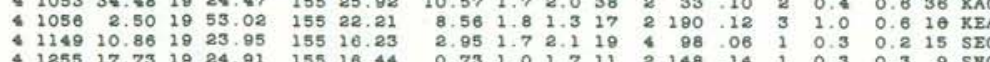

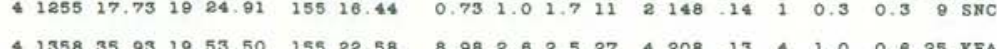

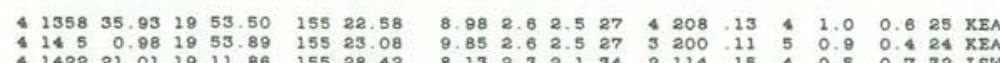

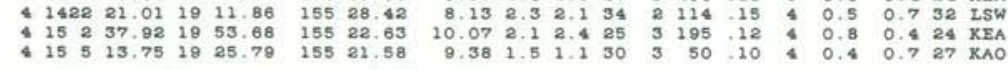

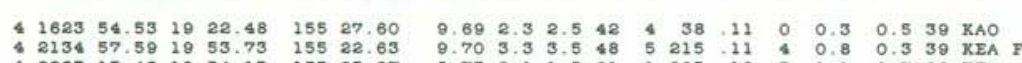

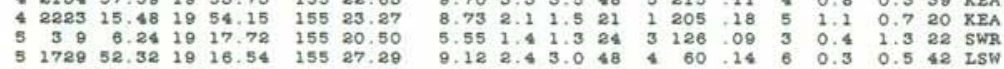

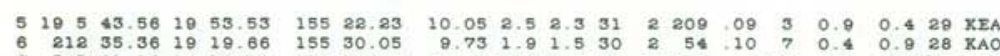

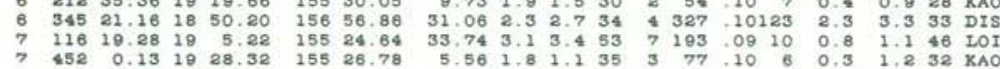

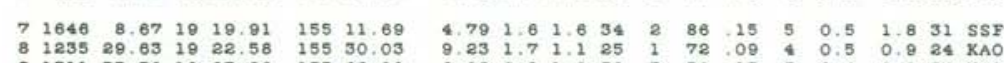

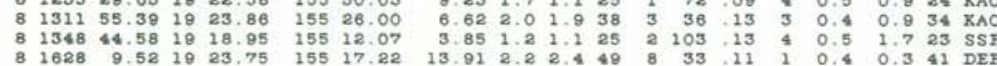

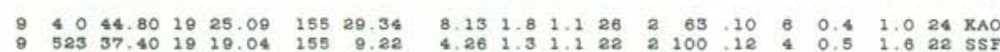

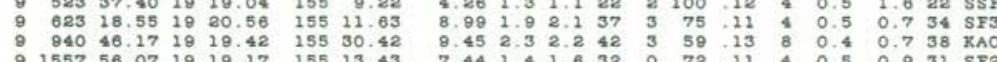

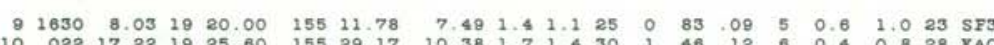

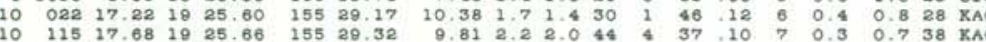

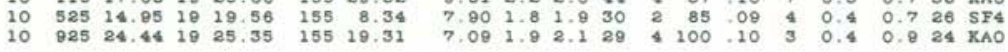
$\begin{array}{rrrrrrrrrrrrrrrrrrrrr}10 & 1448 & 34.58 & 10 & 22.51 & 155 & 28.65 & 8.78 & 1.6 & 1.7 & 34 & 1 & 70 & 12 & 4 & 0.4 & 0.8 & 33 & \text { KAO } \\ 10 & 2056 & 17.92 & 10 & 26.63 & 155 & 31.41 & 12.30 & 2.2 & 2.0 & 39 & 4 & 35 & 11 & 7 & 0.3 & 0.5 & 36 & \text { XAO }\end{array}$

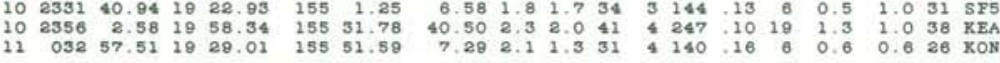

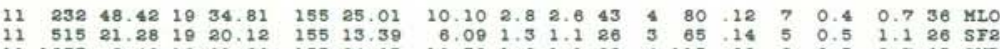

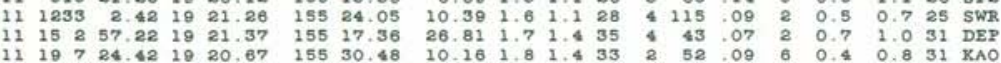

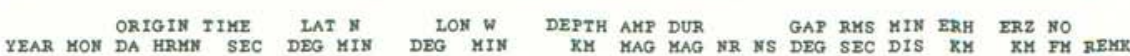

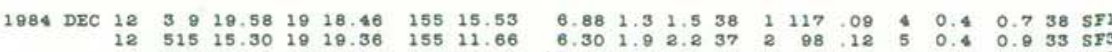

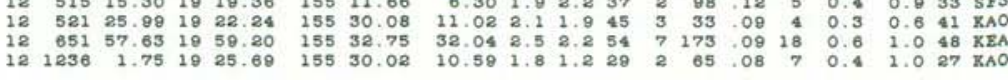

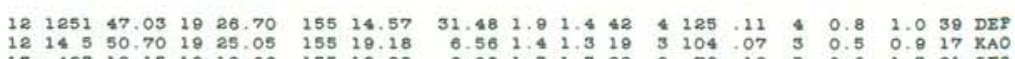

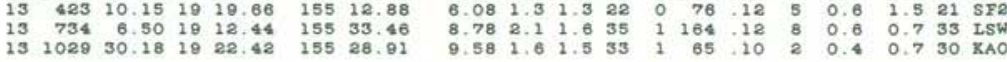

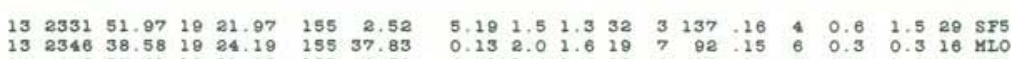

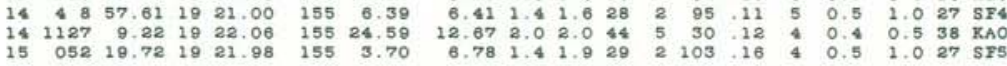

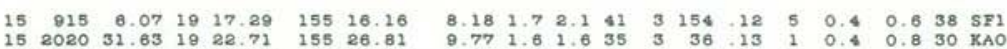

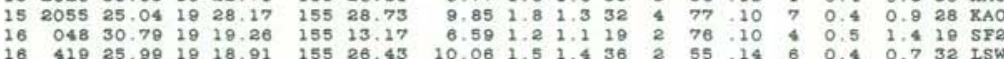

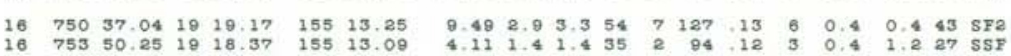

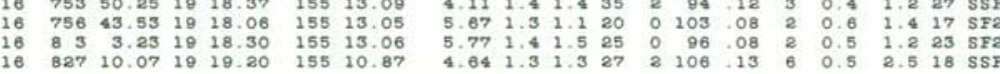

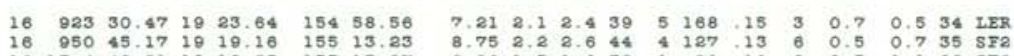

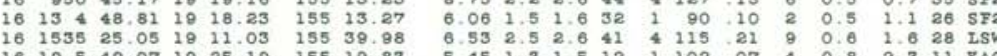

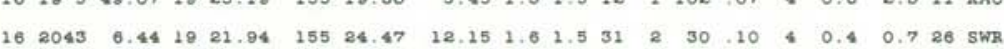

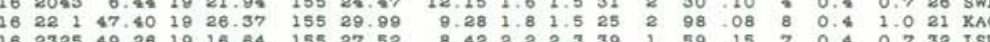

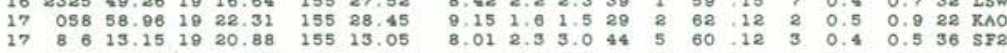
$\begin{array}{lllllllllllllllllll}17 & 1052 & 39.33 & 19 & 20.16 & 155 & 13.01 & 4.80 & 1.0 & 1.1 & 21 & 0 & 68 & 13 & 5 & 0.6 & 2.1 & 19 & \text { SSF } \\ 17 & 1117 & 14.48 & 19 & 17.59 & 155 & 30.34 & 3.77 & 2.3 & 2.6 & 43 & 3 & 45 & 21 & 5 & 0.4 & 1.9 & 38 & \text { LSW }\end{array}$

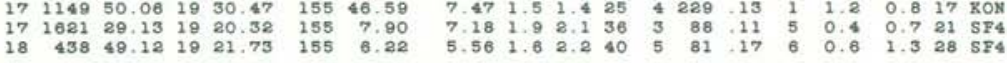

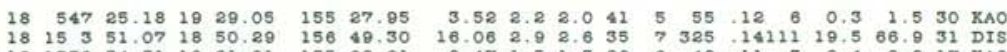

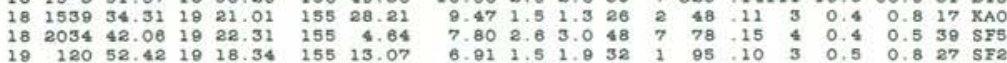




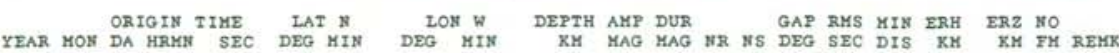

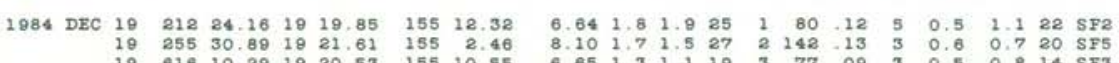

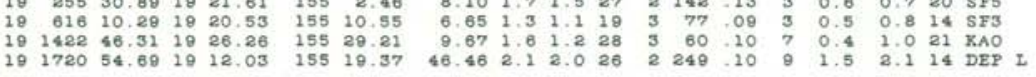

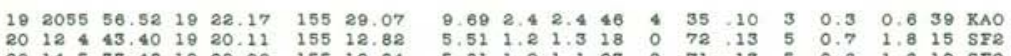

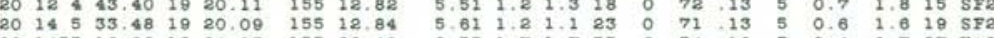

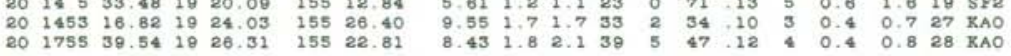

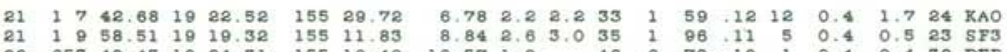

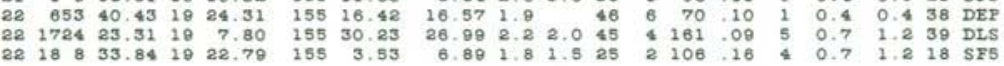
$\begin{array}{llllllllllllllllllll}22 & 2028 & 12.62 & 19 & 21.82 & 155 & 4.02 & 5.68 & 1.7 & 1.1 & 29 & 3 & 94 & 19 & 4 & 0.7 & 1.4 & 18 & \text { SF5 } \\ 22 & 2341 & 40.80 & 19 & 20.71 & 155 & 11.44 & 7.46 & 1.6 & 1.8 & 38 & 5 & 73 & 12 & 4 & 0.5 & 0.6 & 28 & \text { SF3 }\end{array}$

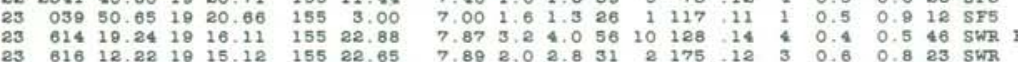

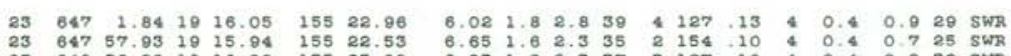

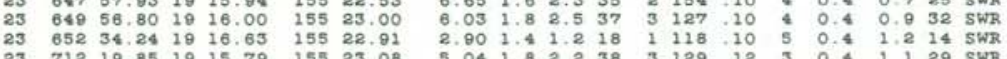

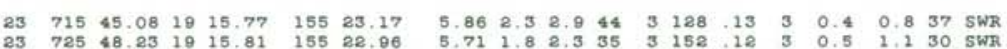

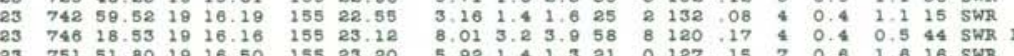

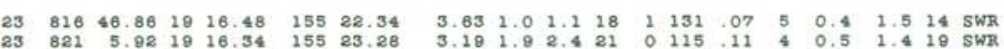

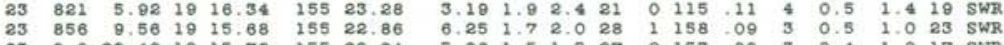

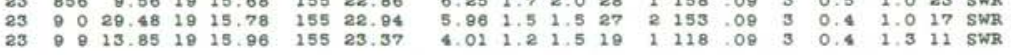

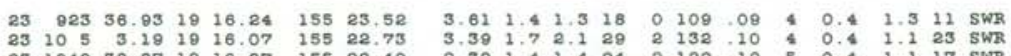

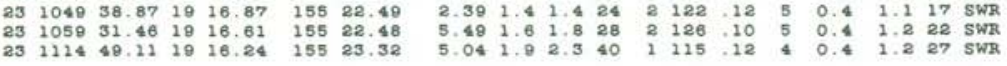
$\begin{array}{lllllllllllllllllll}23 & 1226 & 31.44 & 19 & 16.58 & 155 & 22.88 & 2.56 & 1.4 & 1.3 & 22 & 1 & 119 & 11 & 5 & 0.4 & 1.2 & 15 & \text { sWR } \\ 23 & 1337 & 53.49 & 19 & 16.01 & 155 & 22.87 & 3.30 & 1.4 & 1.2 & 16 & 1 & 150 & 07 & 4 & 0.5 & 1.2 & 15 & \text { SWR }\end{array}$

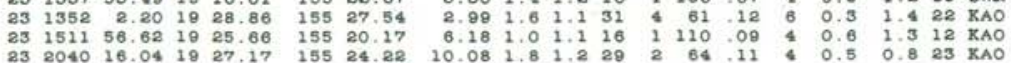

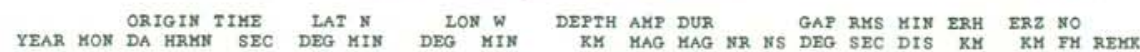
$\begin{array}{lllllllllllllllllll}1984 \text { DEC } 23 & 2117 & 45.16 & 19 & 19.59 & 155 & 28.31 & 9.25 & 1.5 & 1.3 & 22 & 2 & 60 & 12 & 6 & 0.5 & 0.7 & 13 & \text { KAO }\end{array}$

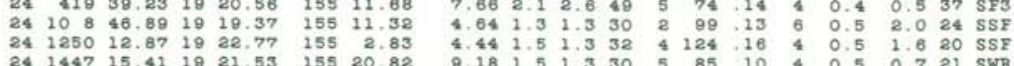
24.

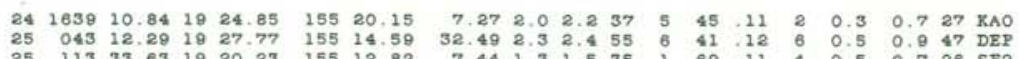

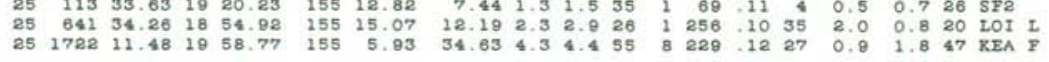

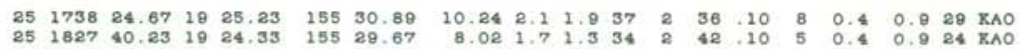

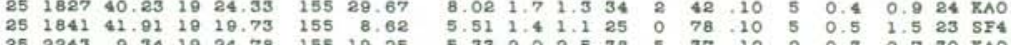

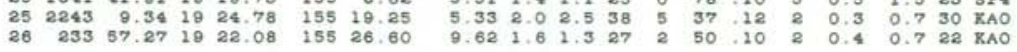

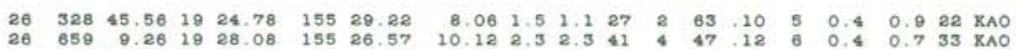

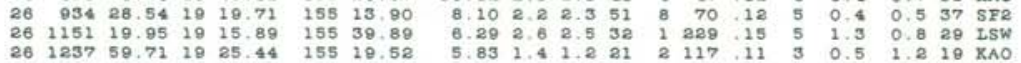

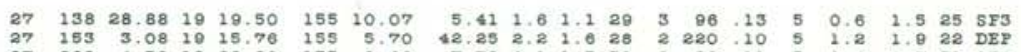

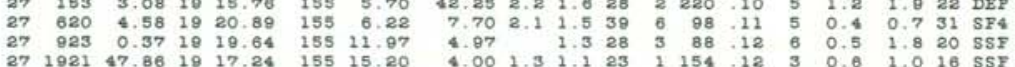

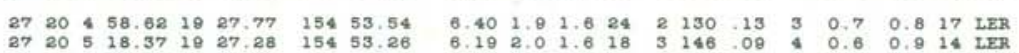

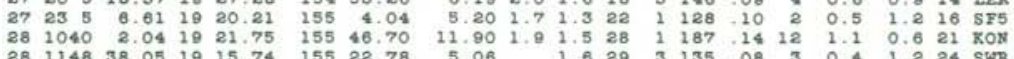

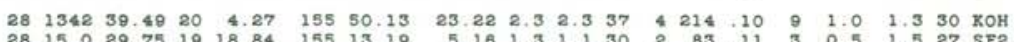

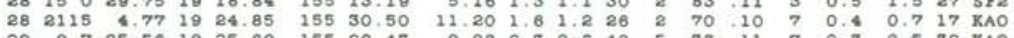

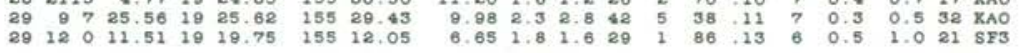

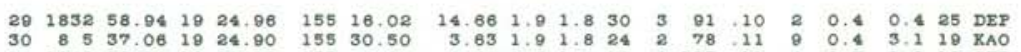

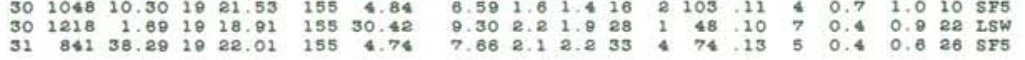

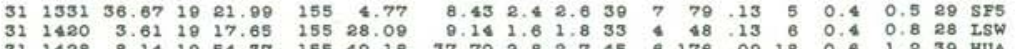


ORIGIN TIME LAT N LON $W$ YEAR MON DA HRMN SEC DEG MIN DEG MIN

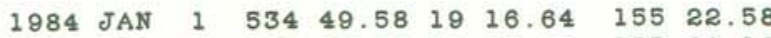

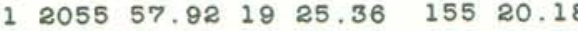
$\begin{array}{lllllll}2 & 1410 & 20.10 & 20 & 35.30 & 154 & 54.93\end{array}$ $\begin{array}{llllllll}12 & 1032 & 27.79 & 19 & 18.54 & 155 & 14.20\end{array}$ $\begin{array}{llllllll}1748 & 15 & 72 & 19 & 18 & 34 & 155 & 14.10\end{array}$

$\begin{array}{lllllll}13 & 1222 & 36.18 & 19 & 27.78 & 155 & 23.40\end{array}$ 10 (1) $48.1801027 .00 \quad 155 \quad 7.20$ $19214.48 .621922 .00 \quad 1554.20$ 2225135.371920 .451552 .75 2871620.741925 .9815010 .80 $\begin{array}{llllll}28 & 823 & 7.57 & 19 & 38.66\end{array}$ $\begin{array}{llllll}30 & 2026 & 56.47 & 19 & 33.93\end{array}$

. 1960.15 $\begin{array}{llllll}2 & 1551 & 22.49 & 19 & 23.71\end{array}$ $\begin{array}{lllll}3 & 622 & 25.10 & 19 & 18.25\end{array}$

$\begin{array}{llllll}3 & 1945 & 35.89 & 19 & 11.53\end{array}$ $\begin{array}{lllll}12 & 1618 & 11.21 & 19 & 16.13\end{array}$ $\begin{array}{llllll}13 & 1027 & 21.80 & 19 & 23.98\end{array}$ $\begin{array}{llllll}13 & 2110 & 12.47 & 30 & 58.05\end{array}$ $\begin{array}{llllll}15 & 722 & 41 & 10 & 19 & 23.96\end{array}$

$\begin{array}{llllll}16 & 652 & 33.13 & 19 & 28.85\end{array}$ $17 \quad 245 \quad 6.28 \quad 1928.85$ $3350-50-1025.08$ 2815200.341000 .03

$\begin{array}{lllll}914 & 37.49 & 19 & 12.72\end{array}$

$\begin{array}{lllllll}8 & 1058 & 49.88 & 19 & 19.32\end{array}$ $1104848.0310 \quad 40.28$ 1) $154 \quad 46.0319 \quad 49.28$ $13 \quad 1142 \quad 15.96 \quad 19 \quad 15.58$

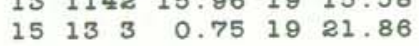

$\begin{array}{lllll}17 & 1927 & 35.08 & 19 & 20.01\end{array}$ $\begin{array}{lllll}21 & 2228 & 24.65 & 19 & 25.64\end{array}$ $25 \quad 050 \quad 52.75 \quad 19 \quad 28.40$ $\begin{array}{llllll}25 & 055 & 13.58 & 19 & 27.80\end{array}$ $\begin{array}{llllll}25 & 1 & 0 & 22.15 & 19 & 28.02\end{array}$

$\begin{array}{lllllll}25 & 1 & 1 & 20.34 & 19 & 27.38\end{array}$

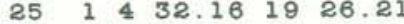
$\begin{array}{llllll}25 & 1 & 8 & 8.54 & 19 & 27.10\end{array}$ $\begin{array}{llllll}25 & 210 & 24.72 & 19 & 28.68\end{array}$ $\begin{array}{lllllll}25 & 21 & 5 & 50.33 & 19 & 27.76\end{array}$

$\begin{array}{lllll}27 & 914 & 42.82 & 19 & 21.15\end{array}$ $\begin{array}{llllll}30 & 1021 & 15.12 & 19 & 58.21\end{array}$

$\begin{array}{llllll}A P R & 1 & 222 & 25.03 & 19 & 27.28\end{array}$

$\begin{array}{llllll}6 & 1843 & 29.04 & 19 & 18.71\end{array}$

$7539.161928,10$
DEPTH AMP DUR

GAP RMS MIN ERH ERZ NO RY MAG MAG NR NS DEG SEC DIS KM KM FM REMR

$\begin{array}{lllllllllllll}8.03 & 2.7 & 3.4 & 49 & 1 & 124 & .17 & 5 & 0.5 & 0.7 & 48 & \text { SWR }\end{array}$ $\begin{array}{llllllllllll}7.76 & 3.0 & 3.3 & 51 & 6 & 38 & .12 & 3 & 0.3 & 0.6 & 45 & \text { KAO }\end{array}$

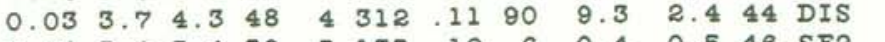
$\begin{array}{llllllllllll}9.48 & 3.2 & 3.4 & 50 & 5 & 135 & .12 & 6 & 0.4 & 0.5 & 46 & \text { SF2 }\end{array}$ $\begin{array}{llllllllllll}10.01 & 3.4 & 3.7 & 48 & 4 & 137 & .13 & 7 & 0.5 & 0.5 & 43 & \text { SF2 }\end{array}$

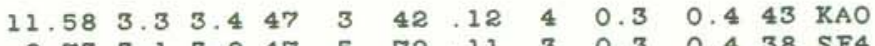
$\begin{array}{lllllllllllll}8.73 & 3.1 & 3.2 & 47 & 5 & 72 & .11 & 3 & 0.3 & 0.4 & 38 & \text { SF4 }\end{array}$ $\begin{array}{rrrrrrrrrrrr}8.12 & 2.9 & 3.1 & 33 & 3 & 196 & .11 & 9 & 0.9 & 0.7 & 24 & \text { SF5 }\end{array}$

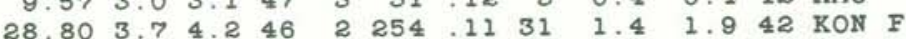
$\begin{array}{lllllllllllll}43.05 & 3.0 & 3.2 & 52 & 7 & 231 & .09 & 22 & 0.7 & 1.3 & 45 & \mathrm{KON}\end{array}$ $\begin{array}{llllllllllll}15.67 & 3.4 & 3.7 & 48 & 6 & 229 & 10 & 14 & 1.0 & 0.7 & 39 & \mathrm{KON}\end{array}$

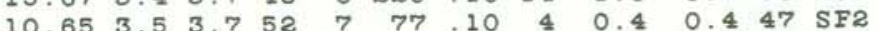
$\begin{array}{lllllllllllll}10.65 & 3.5 & 3.7 & 52 & 7 & 77 & 10 & 4 & 0.4 & 0.7 & 49 & \text { MLO }\end{array}$ $\begin{array}{llllllllllll}10.13 & 3.5 & 3.7 & 52 & 6 & 67 & .11 & 2 & 0.4 & 0.4 & 44 & \text { SF2 F }\end{array}$

$\begin{array}{lllllllllllll}9.09 & 3.3 & 3.5 & 52 & 8 & 93 & .23 & 6 & 0.6 & 0.8 & 42 & \text { LSW }\end{array}$ $\begin{array}{llllllllllll}7.93 & 3.2 & 3.2 & 45 & 4 & 81 & 19 & 3 & 0.5 & 0.9 & 41 & \text { LSW }\end{array}$ $\begin{array}{lllllllllllll}9.11 & 3.1 & 3.0 & 50 & 6 & 28 & .16 & 3 & 0.4 & 0.6 & 39 & \mathrm{KAO}\end{array}$ $\begin{array}{llllllllll}29.58 & 6.0 & 45 & 2 & 344 & 15500 & 18.0 & 3.9 & 17 & \text { DIS I }\end{array}$

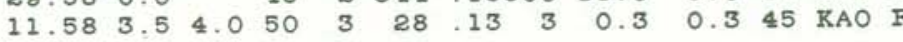

$\begin{array}{llllllllllll}11.97 & 3.2 & 3.1 & 54 & 7 & 41 & .13 & 6 & 0.3 & 0.4 & 46 & \text { KAO }\end{array}$ $\begin{array}{lllllllllll}8.74 & 3.3 & 3.7 & 50 & 9 & 87 & .09 & 3 & 0.4 & 0.4 & 44 \\ 8 F 5\end{array}$

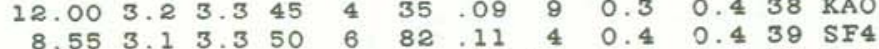
$\begin{array}{llllllllllll}1.47 & 3.3 & 3.2 & 53 & 8 & 84 & .20 & 10 & 0.5 & 0.8 & 39 & \text { LSW }\end{array}$

$\begin{array}{lllllllllllll}8.81 & 3.5 & 3.9 & 46 & 4 & 98 & .10 & 5 & 0.4 & 0.3 & 42 & \text { SF3 } & F\end{array}$ \begin{tabular}{llllllllllll}
18.50 & 3.1 & 3.4 & 52 & 7 & 101 & 11 & 6 & 0.5 & 1.5 & 43 & $\mathrm{KEA}$ \\
\hline
\end{tabular}

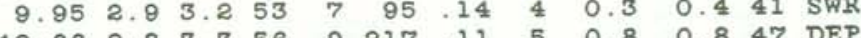

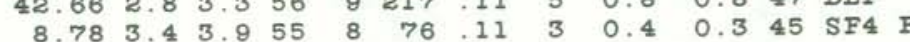

$\begin{array}{llllllllllll}9.41 & 3.1 & 3.5 & 46 & 3 & 82 & .11 & 5 & 0.3 & 0.4 & 40 & \text { SF3 }\end{array}$ $\begin{array}{llllllllllll}2.45 & 3.2 & 3.5 & 42 & 3 & 77 & 12 & 4 & 0.4 & 0.8 & 35 & \text { MLO }\end{array}$

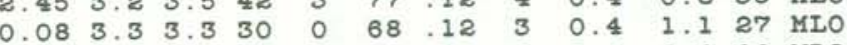

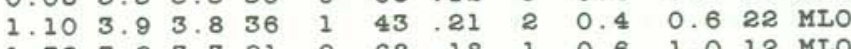
$\begin{array}{llllllllllll}1.52 & 3.2 & 3.3 & 21 & 0 & 68 & 18 & 1 & 0.6 & 1.0 & 12 & \text { MLO }\end{array}$

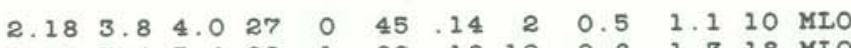

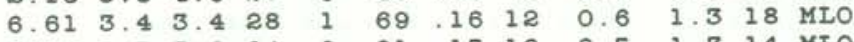

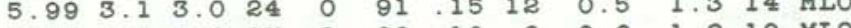

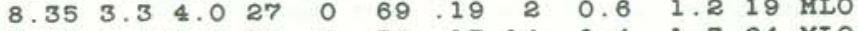
$\begin{array}{llllllllllll}5.91 & 3.3 & 3.9 & 29 & 1 & 54 & .13 & 14 & 0.4 & 1.3 & 24 & \text { MLO }\end{array}$

$\begin{array}{lllllllllll}6.70 & 3.1 & 3.0 & 40 & 3 & 44 & .13 & 4 & 0.3 & 0.7 & 33 \\ \mathrm{KAO}\end{array}$ $\begin{array}{llllllllllll}35.58 & 3.8 & 3.9 & 58 & 10 & 229 & .12 & 28 & 0.9 & 1.4 & 48 & \mathrm{KEA}\end{array}$ $\begin{array}{lllllllllll}10.11 & 3.2 & 2.9 & 55 & 8 & 35 & .12 & 9 & 0.3 & 0.4 & 44 \\ \mathrm{KAO}\end{array}$ $\begin{array}{llllllllllll}10.04 & 3.4 & 3.6 & 53 & 7 & 91 & .12 & 3 & 0.5 & 0.4 & 47 & \text { SF2 }\end{array}$ $\begin{array}{llllllllllll}1.92 & 3.7 & 3.9 & 48 & 3 & 78 & .14 & 2 & 0.3 & 0.3 & 46 & \text { MLO }\end{array}$ 

YEAR MON DA HRMN SEC DEG MIN

1984 APR $\begin{array}{rrrrr}10 & 1137 & 2.66 & 19 & 10.60 \\ 18 & 640 & 13.00 & 19 & 17.88 \\ 22 & 223 & 29.03 & 19 & 18.32\end{array}$ $\begin{array}{lllll}22 & 223 & 29.03 & 19 & 18.32\end{array}$ $\begin{array}{lllll}22 & 227 & 3.59 & 19 & 18.44\end{array}$ $27 \begin{array}{lllll}2348 & 49.48 & 19 & 19.18\end{array}$ $\begin{array}{lllll}28 & 2045 & 34.42 & 19 & 10.40\end{array}$ $\begin{array}{llllll}30 & 1349 & 34.24 & 19 & 43.42\end{array}$

$\begin{array}{lllllll}\text { MAY } & 1 & 355 & 23.08 & 19 & 12.67\end{array}$ $\begin{array}{llllll}9 & 1545 & 10.92 & 19 & 22.28\end{array}$

$\begin{array}{lllll}10 & 926 & 58.75 & 19 & 59.20\end{array}$ $\begin{array}{lllll}17 & 241 & 10.52 & 19 & 21.46\end{array}$ $\begin{array}{lllll}19 & 1418 & 1.67 & 19 & 19.34\end{array}$ $\begin{array}{llllll}19 & 1841 & 2.66 & 19 & 20.55\end{array}$ $20 \quad 533 \quad 19.45 \quad 19 \quad 19.68$

$\begin{array}{lllllll}30 & 18 & 73.60 & 19 & 28.98\end{array}$ $\begin{array}{llllll}31 & 2057 & 37.17 & 19 & 25.44\end{array}$ JUN $\begin{array}{llllll}3 & 2112 & 7.46 & 18 & 54.25\end{array}$ $\begin{array}{lllllll}8 & 927 & 50.72 & 19 & 12.06\end{array}$ $\begin{array}{llllll}8 & 933 & 12.30 & 19 & 12.10\end{array}$

$\begin{array}{llllll}8 & 1734 & 10.64 & 20 & 3.31\end{array}$ $\begin{array}{lllll}12 & 1339 & 0.35 & 19 & 37.43\end{array}$ $\begin{array}{llllll}12 & 17 & 3 & 20.35 & 19 & 37.43 \\ 19 & 19 & 21.09\end{array}$

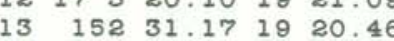
$16 \quad 822 \quad 10.03$ 20 44.51 $\begin{array}{llllll}17 & 11 & 5 & 23.53 & 19 & 21.42\end{array}$ $\begin{array}{llllll}20 & 919 & 36.81 & 19 & 21.28\end{array}$ $\begin{array}{lllll}24 & 2123 & 19.96 & 19 & 20.20\end{array}$ $\begin{array}{lllll}25 & 1329 & 43.71 & 19 & 14.81\end{array}$ $26 \quad 1914 \quad 31.2720 \quad 1.05$

$\begin{array}{llllll}30 & 414 & 45.00 & 19 & 20.49\end{array}$

$\begin{array}{llllll}\text { JUI } & 4 & 1430 & 13.23 & 19 & 0.48\end{array}$ $\begin{array}{llllll}8 & 1726 & 33.95 & 21 & 33.09\end{array}$ $\begin{array}{lllllll}20 & 847 & 47.93 & 20 & 2.98\end{array}$ $\begin{array}{lllllll}25 & 832 & 38 & 15 & 19 & 22.16\end{array}$ $\begin{array}{lllllll}26 & 545 & 11.12 & 19 & 21.11\end{array}$

AUG $3 \quad 5 \quad 8 \quad 39.32 \quad 19 \quad 19.56$ $\begin{array}{llllll}10 & 2145 & 45.26 & 19 & 6.65\end{array}$ $\begin{array}{lllll}11 & 2329 & 30.15 & 19 & 27.4\end{array}$ $141010 \quad 2.1219 \quad 47.1$

$\begin{array}{llllll}18 & 010 & 52.36 & 19 & 21.65\end{array}$ $\begin{array}{llllll}24 & 028 & 32.72 & 19 & 22.18\end{array}$ $\begin{array}{llllll}28 & 728 & 48.88 & 19 & 12.34\end{array}$ $\begin{array}{lllll}31 & 1333 & 31.09 & 20 & 8.44\end{array}$ $\begin{array}{lllll}31 & 2253 & 23.50 & 19 & 21.85\end{array}$
LON W

DEG MIN

$155 \quad 13.55$ $156 \quad 47.69$ $\begin{array}{ll}155 & 12.95\end{array}$ $155 \quad 13.46$ 15528.38 $\begin{array}{ll}55 & 40.60\end{array}$ $154 \quad 52.79$ $155 \quad 34.14$ 15529.15

$155 \quad 53.90$ $\begin{array}{ll}155 & 2.75\end{array}$ $\begin{array}{ll}155 & 11.37\end{array}$ $\begin{array}{ll}55 & 13.00\end{array}$ 15511.25

$155 \quad 35.70$ $\begin{array}{ll}155 & 19.51\end{array}$ $155 \quad 8.68$ $\begin{array}{lll}55 & 31.22\end{array}$ 15531.20

15752.51 15555.77 15530.11 $\begin{array}{ll}155 & 12.78\end{array}$ 15730.32

\section{$155 \quad 30.10$} $\begin{array}{ll}155 & 29.88\end{array}$ 15510.85 1554.08 $\begin{array}{ll}155 & 21.49\end{array}$

15511.72 $156 \quad 54.19$ 15456.29 $\begin{array}{lll}55 & 43.69\end{array}$ 15518,63

$155 \quad 7.38$ $\begin{array}{lll}155 & 15.46\end{array}$ $\begin{array}{ll}56 & 10.73\end{array}$ 5529.36 $154 \quad 54.77$

\section{$155 \quad 4.84$} $\begin{array}{ll}155 & 2.67\end{array}$ $155 \quad 36.46$ 15550.44 1555.10
DEPTH AMP DUR

GAP RMS MIN ERH

KM MAG MAG NR NS DEG SEC DIS KM KM FM REMK

$\begin{array}{lllllllllllll}10.49 & 3.9 & 4.1 & 56 & 10 & 73 & .11 & 3 & 0.4 & 0.3 & 46 & \text { SF2 } & F\end{array}$ $\begin{array}{llllllllllll}28.41 & 3.4 & 2.6 & 36 & 2 & 305 & .13 & 98 & 1.8 & 3.5 & 34 & \text { DIS }\end{array}$ $\begin{array}{llllllllllll}6.88 & 3.0 & 3.0 & 44 & 5 & 114 & .12 & 2 & 0.4 & 0.8 & 39 & \text { SF2 }\end{array}$

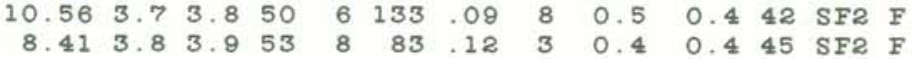

$\begin{array}{llllllllllll}9.68 & 3.2 & 3.4 & 50 & 7 & 41 & .12 & 6 & 0.3 & 0.5 & 41 & \mathrm{KAO}\end{array}$

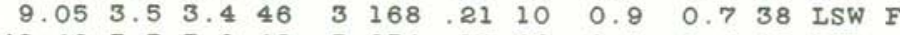

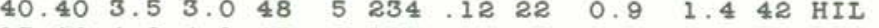
$\begin{array}{rrrrrrrrrrrr}13.22 & 4.0 & 4.1 & 45 & 6 & 123 & .15 & 7 & 0.5 & 0.5 & 39 & \text { DIS F } \\ 10.22 & 3.0 & 3.3 & 57 & 11 & 35 & 11 & 3 & 0.3 & 0.4 & 43 & \text { XAO }\end{array}$

$\begin{array}{llllllllllll}22.35 & 3.0 & 3.0 & 18 & 4 & 221 & .13 & 20 & 1.4 & 3.2 & 11 & \mathrm{KOH}\end{array}$ $\begin{array}{llllllllllll}7.07 & 2.9 & 3.1 & 49 & 6 & 134 & .12 & 3 & 0.4 & 0.5 & 42 & \text { SF5 }\end{array}$ $\begin{array}{llllllllllll}8.85 & 3.0 & 3.4 & 49 & 4 & 100 & .10 & 6 & 0.3 & 0.4 & 42 & \text { SF3 }\end{array}$

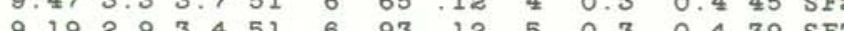

$\begin{array}{llllllllllll}1.58 & 3.0 & 3.5 & 17 & 2 & 71 & .21 & 1 & 0.6 & 0.3 & 9 & \text { MLO }\end{array}$

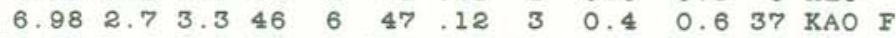
$\begin{array}{llllllllllll}50.20 & 3.4 & 3.3 & 55 & 8 & 254 & 09 & 43 & 0.9 & 1.5 & 47 & \text { LOI }\end{array}$ $\begin{array}{llllllllllll}37.56 & 3.7 & 3.7 & 56 & 10 & 85 & .09 & 6 & 0.6 & 1.0 & 46 & \text { DLS }\end{array}$ $\begin{array}{llllllllllll}37.45 & 3.1 & 3.3 & 57 & 10 & 83 & .10 & 6 & 0.6 & 0.9 & 47 & \text { DLS }\end{array}$

$\begin{array}{lllllllllll}29.55 & 5.0 & 5.3 & 52 & 5 & 238 & .13142 & 1.5 & 2.5 & 41 & \text { DIS }\end{array}$

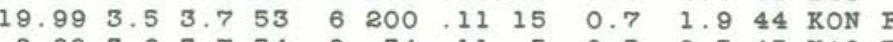

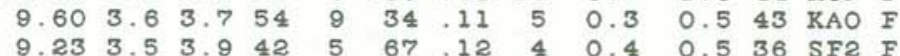

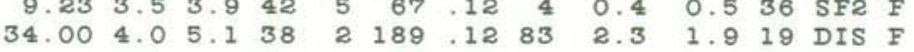

$\begin{array}{llllllllllll}9.69 & 3.1 & 3.4 & 55 & 8 & 33 & .10 & 5 & 0.3 & 0.4 & 44 & \text { KAO }\end{array}$ $\begin{array}{lllllllllll}10.84 & 2.8 & 3.2 & 51 & 6 & 34 & .09 & 5 & 0.3 & 0.4 & 41 \\ \text { KAO }\end{array}$ $\begin{array}{rrrrrrrrrrrrr}9.61 & 3.5 & 3.7 & 49 & 5 & 82 & .09 & 4 & 0.4 & 0.3 & 44 & \text { SF3 } & \text { F }\end{array}$

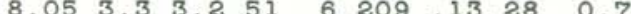

$\begin{array}{llllllllllll}9.06 & 2.9 & 3.3 & 49 & 6 & 75 & .12 & 4 & 0.3 & 0.4 & 41 & \text { SF3 }\end{array}$ $\begin{array}{lllllllllll}28.81 & 3.4 & 4.1 & 30 & 5 & 323 & .12112 & 1.9 & 4.6 & 23 & \text { DIS }\end{array}$ $\begin{array}{lllllllllll}7.30 & 3.5 & 3.8 & 43 & 8 & 328 & 13181 & 1.8 & 4.4 & 23 & \text { DIS }\end{array}$ $\begin{array}{lllllllllllllll}7.41 & 3.6 & 3.9 & 47 & 4 & 144 & .14 & 10 & 0.7 & 0.8 & 40 & \mathrm{KOH} & F\end{array}$ $\begin{array}{lllllllllllll}30.97 & 3.5 & 3.9 & 46 & 1 & 35 & 11 & 3 & 0.6 & 1.0 & 44 & \text { DEP }\end{array}$

$\begin{array}{llllllllllll}8.27 & 3.3 & 3.9 & 48 & 5 & 172 & .12 & 4 & 0.6 & 0.5 & 42 & \mathrm{SF} 4\end{array}$

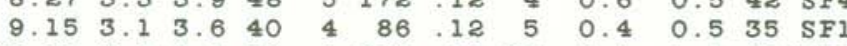

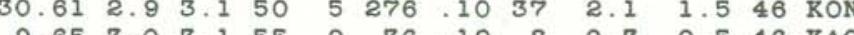

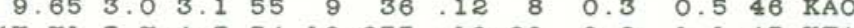
$\begin{array}{llllllllllllllllll}47.71 & 3.7 & 4.3 & 54 & 10 & 233 & 10 & 20 & 0.8 & 1.1 & 43 & \mathrm{KEA} & \end{array}$

$\begin{array}{llllllllllll}7.61 & 2.8 & 3.3 & 48 & 5 & 82 & .12 & 5 & 0.4 & 0.5 & 36 & \text { SF5 }\end{array}$ $\begin{array}{llllllllllll}7.28 & 2.8 & 3.2 & 41 & 6 & 132 & .12 & 4 & 0.5 & 0.6 & 32 & \text { SF5 }\end{array}$

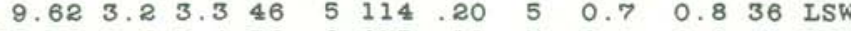
$\begin{array}{llllllllllll}39.07 & 3.3 & 3.4 & 52 & 8 & 275 & .11 & 7 & 0.8 & 0.9 & 44 & \mathrm{KOH}\end{array}$ $\begin{array}{lllllllllllll}8.69 & 2.8 & 3.3 & 48 & 6 & 78 & .10 & 4 & 0.4 & 0.3 & 39 & \text { SF5 }\end{array}$ 
ORIGIN TIME LAT N YEAR MON DA HRMN SEC DEG MIN

$\begin{array}{llllllll}1984 & \text { SEP } & 2 & 1643 & 14.45 & 19 & 21.28\end{array}$ $\begin{array}{llllll}10 & 1916 & 6.49 & 18 & 55.68\end{array}$ $\begin{array}{llllll}10 & 2351 & 36.28 & 19 & 20.59\end{array}$ $\begin{array}{lllll}13 & 2027 & 59.48 & 19 & 27.84\end{array}$ $242127 \quad 48.201918 .97$

$\begin{array}{llllllll}7 & 3 & 12.74 & 19 & 19.40\end{array}$ 6175450.821914 .12 $800025.1120 \quad 7.02$ $\begin{array}{lllllll}8 & 0 & 3 & 43.06 & 19 & 17.09\end{array}$

$8 \quad 1618 \quad 10.72 \quad 10 \quad 54.90$ $\begin{array}{lllll}10 & 233 & 49.75 & 19 & 17.23\end{array}$ $\begin{array}{lllll}13 & 233 & 49.75 & 19 & 17.23 \\ 13 & 616 & 26.92 & 19 & 20.62\end{array}$ $16 \quad 53414.72 \quad 18 \quad 54.59$ $\begin{array}{lllll}16 & 1445 & 55.79 & 19 & 24.47\end{array}$ $\begin{array}{llllll}23 & 331 & 48.64 & 19 & 20.47\end{array}$ $\begin{array}{llllll}29 & 420 & 38.25 & 19 & 14.60\end{array}$ $\begin{array}{lllll}31 & 1922 & 22.46 & 19 & 12.46\end{array}$ NOV $11 \quad 534 \quad 6.20 \quad 18 \quad 55.84$ $\begin{array}{llllll}11 & 550 & 37.04 & 18 & 58.30\end{array}$

$\begin{array}{lllllll}11 & 10 & 5 & 13.85 & 18 & 56.54\end{array}$ $\begin{array}{lllllll}11 & 19 & 3 & 19.55 & 18 & 20.57\end{array}$ $\begin{array}{llllll}4 & 1 & 5 & 56.24 & 19 & 51.79\end{array}$ $\begin{array}{llllll}16 & 1 & 1 & 58.02 & 19 & 22.2\end{array}$

4.233457 .592953 .73 $\begin{array}{lllll}7 & 116 & 19.28 & 19 & 5.22\end{array}$ 1675037.041919 .17 $23 \quad 614 \quad 19.24 \quad 19 \quad 16.1$ $\begin{array}{llllll}23 & 746 & 18.53 & 19 & 16.16\end{array}$

$25172211.48 \quad 1958.77$
ION W

$155 \quad 1.58$ 15516.61 5513.34 $55 \quad 54.17$

15510.99 $\begin{array}{ll}155 & 27.18\end{array}$ 15510.87 $155 \quad 15.48$

$155 \quad 29.41$ 15525.17 1557.78 $155 \quad 15.32$ $155 \quad 29.36$

$\begin{array}{lll}155 & 11.97\end{array}$ 5453.33 $\begin{array}{ll}55 & 26.84 \\ 55 & 13.30\end{array}$ $\begin{array}{lll}155 & 13.87\end{array}$

$\begin{array}{lll}155 & 12.68\end{array}$ 15511.26 $\begin{array}{ll}55 & 8.87\end{array}$ $55 \quad 28.86$
-158 $155 \quad 38,14$

$\begin{array}{ll}55 & 22.63 \\ 2 & -63\end{array}$ $\begin{array}{ll}55 & 13.25\end{array}$ 15522.88 $155 \quad 23.12$

$155 \quad 5.93$
DEPTH AMP DUR

GAP RMS MIN ERH KM MAG MAG NR NS DEG SEC DIS KM $\begin{array}{llll}0.6 & 0.4 & 32 & \text { SF5 }\end{array}$ $\begin{array}{llllllllllll}14.62 & 3.1 & 4.1 & 43 & 0 & 244 & 10 & 32 & 1.7 & 2.0 & 34 & \text { LOI I } \\ 9.04 & 3.4 & 3.6 & 46 & 5 & 60 & 12 & 4 & 0.4 & 0.5 & 40 & \text { SF2 }\end{array}$

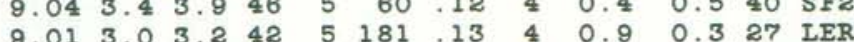

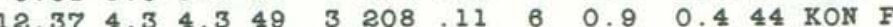

$\begin{array}{llllllllllllll}9.63 & 3.2 & 3.7 & 51 & 7 & 100 & .10 & 5 & 0.5 & 0.4 & 43 & \text { SF3 } F\end{array}$ $\begin{array}{llllllllllll}13.70 & 3.1 & 3.3 & 54 & 8 & 103 & .13 & 5 & 0.4 & 0.4 & 45 & \text { DLS }\end{array}$ $\begin{array}{llllllllllll}31.70 & 3.0 & 3.0 & 57 & 9 & 199 & 12 & 11 & 0.8 & 1.0 & 46 & \text { DEP }\end{array}$

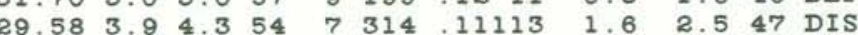
$\begin{array}{llllllllllll}10.10 & 2.9 & 3.1 & 48 & 5 & 151 & 11 & 6 & 0.4 & 0.4 & 42 & \text { SF1 }\end{array}$

$\begin{array}{llllllllllllll}17.85 & 3.7 & 4.1 & 56 & 9 & 151 & .11 & 15 & 0.6 & 1.8 & 47 & \text { KEA } & F\end{array}$

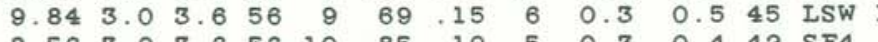

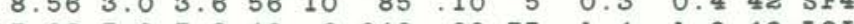

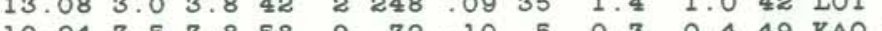

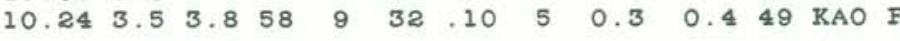
$\begin{array}{llllllllllll}8.76 & 3.0 & 3.6 & 49 & 6 & 74 & .11 & 4 & 0.3 & 0.4 & 44 & \text { SF3 }\end{array}$

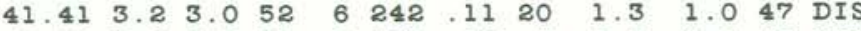
$\begin{array}{llllllllllll}9.32 & 3.0 & 3.3 & 55 & 8 & 125 & .17 & 6 & 0.5 & 0.4 & 48 & \text { LSW }\end{array}$ $\begin{array}{lllllllllllll}8.85 & 4.0 & 4.2 & 47 & 7 & 244 & .12 & 36 & 0.9 & 0.6 & 39 & \text { LOI } F\end{array}$ $\begin{array}{lllllllllllll}14.26 & 2.8 & 3.6 & 36 & 3 & 236 & .11 & 32 & 1.3 & 1.4 & 33 & \text { LOI }\end{array}$

$\begin{array}{llllllllllll}10.30 & 2.9 & 3.5 & 41 & 7 & 243 & .12 & 36 & 0.9 & 0.6 & 37 & \text { LOI }\end{array}$ $\begin{array}{llllllllllll}9.06 & 3.4 & 3.9 & 44 & 4 & 167 & .12 & 4 & 0.6 & 0.5 & 35 & \text { SF3 }\end{array}$ $15.693 .8 \quad 3.8 \quad 53 \quad 10 \quad 222.10 \quad 21 \quad 0.9 \quad 1.0 \quad 45 \quad \mathrm{KEA} F$

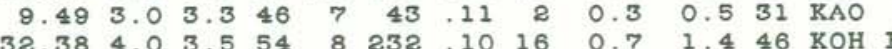
$\begin{array}{lllllllllllll}9.70 & 3.3 & 3.5 & 48 & 5 & 215 & .11 & 4 & 0.8 & 0.3 & 39 & \text { KEA } F\end{array}$ $\begin{array}{lllllllllllll}33.74 & 3.1 & 3.4 & 53 & 7 & 193 & .09 & 10 & 0.8 & 1.1 & 46 & \text { LOI }\end{array}$ $\begin{array}{llllllllllllll}9.49 & 2.9 & 3.3 & 54 & 7 & 127 & 13 & 6 & 0.4 & 0.4 & 43 & \text { SF2 }\end{array}$

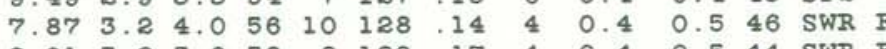
$\begin{array}{lllllllllllll}8.01 & 3.2 & 3.9 & 58 & 8 & 120 & .17 & 4 & 0.4 & 0.5 & 44 & \text { SWR } F\end{array}$

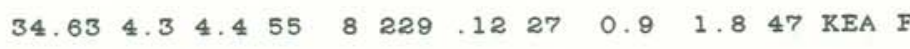


Publication Summary: Hawailan Volcano Observa tory personnel, 1984

Billingsley, G. H., Barnes, C. W., and Ulrich, G. E., (In press), Geologic map of the Coconino Point and Grandview Point quadrangles, Coconino County, Arizona: U.S. Geological Survey Miscellaneous Investigations Map I-___, scale 1:62,500.

Camp, V.A., Hooper, P.R., Swanson, D.A., and Wright, T.L., 1984, Columbia River Basalt in Idaho: Physical and Chemical Characteristics, Flow Distribution, and Tectonic Implications: In Cenezoic Geology of Idaho, Bill Bonnersen and Roy M. Breckinridge (eds.), Bulletin 26, Idaho Department of Lands, Bureau of Mines and Geology, p. 55-75.

Decker, R.W., Koyanag1, R.Y., Dvorak, J.J., Lockwood, J.P., Okamura, A.T., Yamashita, K.M., and Tanigawa, W.R., 1984, Seismicity and ground deformation of Mauna Loa volcano, Hawa11 (abs.): 27 th International Geology Congress (Moscow) Abstracts, v. 4, Sect. 08, 09, p. 59.

Dieterich, J.H., and Okamura, A.T., 1984, Modeling surface deformation due to coupled interactions between magma bodies and fault slip (abs.): in Programs and Abstracts, Chapman Conference on Vertical Crustal Motion: Measurement and Modeling, Harpers Ferry, WV, October 1984.

Dvorak, J., Okamura, A.T., Engl1sh, T.T., Koyanag1, R.Y., Naka ta, J.S., Sako, M.K., Tanigawa, W.R., and Yamashita, K.M., in press, Mechanical response of the south flank of Kilauea Volcano, Hawail to intrusive events along the rift zones: Tectonophysics, $52 \mathrm{~ms}$ p.

Dvorak, J.J., Okamura, A.T., Johnston, M.J.S., Mortensen, C., Mueller, R.J., and Furukawa, B., 1984, T11tmeter and magnetometer measurements at Mount St. Helens, Washington: 1980-1981: U.S. Geol. Survey OpenFile Report 80-164, 46 p.

Dvorak, J.J. and Okamura, A.T., 1984, Subsidence mechanics of the summit regions of shield volcanoes, Hawall (abs.): Eos, Trans., Am. Geophys. Union, v. 65, p. 1138 .

Dvorak, J.J., and Okamura, A.T., 1984, Vertical ground movement associated with volcanism in Hawa11 (abs.): in Programs and Abstracts, Chapman Conference on Vertical Crustal Motion: Measurement and Modeling, Harpers Ferry, WV, October 1984.

Dzurisin, D., Koyanagi, R.Y., English, T.T., 1984, Magma supply and storage at Kilauea volcano, Hawa11, 1956-1983. Journal of Volcanology and Geothermal Research, v. 21, p. 177-206.

Edwards, Kathleen, Sanchez, E. M., and Ulrich, G. E., 1984, Landsat vertical image yields a stereo oblique: Geotimes, v. 29, n. 4, p. 10-11; reprinted in Earth Science, v. 37, n. 4, p. 18-19.

Garcia, M.O., Byers, C.D., Wolfe, E.W., and Rhodes, J.M., 1984, Chemistry and mineralogy of lavas from the continuing eruption of Kilauea volcano, Hawa11 (abs.): Eos, Transactions, American Geophysical Union, v. 65 , p. 1130 . 
Greenland, L.P., 1984, Gas composition of the January 1983 eruption of Kilauea volcano, Hawa11: Geochim. Cosmochima Acta, v. 48, p.193-195.

Greenland, L.P., 1984, Eruptive gases from Mauna Loa and Kilauea volcanoes, Hawa11 (abs.): 1984 Int. Chem. Congr. Pac. Bas. Soc., Honolulu.

Greenland, L.P., and Aruscavage, P., 1984, Volcanic emission of Se, Te, and As: Dir. App., subm. J. Volcan. Geoth. Res.

Greenland, L.P., Casadeval1, T.J., and Stokes, J.B., 1984, Emission rate of $\mathrm{CO}_{2}$ and $\mathrm{SO}_{2}$ from Kilauea volcano, Hawa11: Dir. App., subm. Na ture.

Greenland, L.P., Okamura, A.T., and Stokes, J.B., in press, Contraints on the mechanics of eruption of Pu'u O'o: U.S. Geological Survey Professional Paper

Heliker, C.C., Johnson, Arthur, and Hodges, S.M., 1984, The Nisqually Glacier, Mount Rainier, Washington, 1857-1979: A summary of the long-term observations and a comprehensive bibliography: U.S. Geological Survey Open-File Report 83-541, 14 p.

Heliker, C. Christina, 1984, Inclusions in the 1980-83 dacite of Mount St. Helens, Washington: Western Washington University, unpublished M.S. thesis, $185 \mathrm{p}$.

Helz, R.T., Banks, N.G., Casadevall, T.J., Fiske, R.S., and Moore, R.B., 1984, A catalogue of drill core recovered from Kilauea Iki lava lake from 1967 to 1979: U.S. Geol. Survey Open-File Report 84-0484.

Hoffmann, J.P., Ulrich, G.E., and Chadwick, W.W., 1984, Geodetic Measurements on the east rift zone, Kilauea volcano (abs.): EOS, Transactions of American Geophysical Union, v. 65, p. 1131.

Jackson, D.B., Kauahikaua, J., Zablock1, C.J., in press, Resistivity monitoring of an active volcano using the controlled-source electromagnetic technique: Kilauea Hawail: Journal of Geophysical Research.

Kauahikaua, J., Jackson, D.B., Zablocki, C.J. in press, The subsurface resistivity structure of Kilauea volcano, Hawa11: JHournal of Geophysical Research.

Koyanag1, R.Y., Endo, E.Y., Tanigawa, W.R., Nakata, J.S., Tomori, A.H., Tamura, P.N., 1984. Kaoik1, Hawa1i Earthquake of November 16, 1983: A preliminary compilation of seismographic data at the Hawailan Volcano Observatory. In review of U.S.G.S. Open-f1le Report 84-798, 35 pages.

Koyanag1, R.Y., and Kojima, G., Evaluation of the radio-telemetered seismic network at Merap1 Volcano Observatory, August, 1984. (Administrative report sent to office of International Geology, 1984).

Lipman, P.W., and Banks, N.G. (In press), A'a flow dynamics, Mauna Loa 1984: U.S. Geological Survey Professional Paper 1350. 
Lockwood, J.P., 1984, Review of "Cooke-Ravian volume of volcanological papers": American Mineralogist, v. 69 , n. 4, p. 819.

Lockwood, J.P., 1984, Geologic map of Mauna Loa volcano, Hawa11 -- Kulan1 Quadrangle: U.S. Geological Survey Open-File Report 84-12, scale $1: 24,000$.

Lockwood, J.P., Decker, R.W., Jackson, D.B., Koyanag1, R.Y., Okamura, A.T., and Johnson, D.J., 1984, The 1984 eruption of Mauna Loa volcano, Hawa11 (abs.): American Geophysical Union Transactions, v. , n. , p.

Lockwood, J.P., Decker, R.W., Jackson, D.B., Koyanagi, R.Y., Okamura, A.T., and Johnson, D.J., 1984, The 1984 Eruption of Mauna Loa, Hawa11 (abs.): Eos, Trans., Am. Geophys. Union, v. 65, p. 1137.

Mangan, M., and Wright, T.L., 1984. Regional correlation of Grande Ronde Basalt Flows, Columbia River Basalt Group (abs.): Eos, v. 65, p. 1153.

McClelland, L., and Wolfe, E.W., 198-, The eruption of K1lauea Volcano, Hawa11, January-July 1983: McGraw-H111 Encyclopedia of Sclence 1985 Yearbook. (approved fall 1983).

Nakata, J.S., Tomori, A.H., Koyanag1, R.Y., Tanigawa, R.W., 1984. Hawa11an Volcano Observatory Summary 83, Seismic Data January to December 1983.

Okamura, A.T., Dvorak, J.J., Sako, M.K., Hanatan1, R.Y., Johnson, D.J., and Yamashita, K.M., 1984, Surface deformation associated with the March 25-April 15, 1984, Mauna Loa eruption (abs.): Eos, Trans., Am. Geophys. Union, v. 64, p. 1137.

Sako, M. and Jackson, D.B., 1984, Deformation report on Pagan Volcano: Report submitted to Pedro Tenorio, Governor, Commonwealth of the Northern Marianas.

Tanaka, K.L., Shoemaker, E.M., Ulrich, G.E., and Wolfe, E.W., Migration of volcanism in the San Francsico volcanic field, Arizona: Geological Society of America Bulletin, in press, $44 \mathrm{~ms}$ pg. Branch Chief app. (As trogeology) August 27, 1984.

Ulrich, G.E., Approved for the Director $12 / 6 / 84$, SP Mountain cinder cone Crater and lava flow, northern Arizona: Geol. Soc. of Am. Guide to 100 Localities in the Rocky Mountain Area. $6 \mathrm{~ms}$ pages.

Ulrich, G.E., and Bailey, N.G., in press, Geologic map of the SP Mountain part of the San Francisco volcanic field, Arizona: U.S. Geological Survey Miscellaneous Investigations Map I, scale 1:50,000.

Ulrich, G. E., and Bielski, A. M., 1984, Wet Beaver Roadless Area, Arizona in Marsh, S. P., Kropschot, S. J., and Dickinson, R. G., eds., Wilderness Mineral Potential: assessment of mineral-resource potential in U.S. Forest Service Lands studied 1964-1984: U.S. Geological Survey Professional Paper 1300, p. 123-125. 
Ulrich, G. E., and Bielski, A. M., 1984, West Clear Creek Roadless Area, Arizona in Marsh, S. P., Kropschot, S. J., and Dickinson, R. G., eds., Wilderness Mineral Potential: assessment of mineral-resource potential in U.S. Forest Service Lands studied 1964-1984: U.S. Geological Survey Professional Paper 1300, p. 120-122.

Ulrich, G.E., Billingsley, G.H., Hereford, R., Wolfe, E.W., Nealey, L.D. and Sutton, R.L. 1984, Geology, structure and uranium deposits of the Flagstaff $1^{\circ} \times 2^{\circ}$ quadrangle, Arizona: U.S. Geological Survey Miscellaneous Investigations Map I-1446. (Brief descriptive text.)

Ulrich, G.E., Wolfe, E.W., Hoffmann, J.P., and Neal, C.A., 1984, Continuing eruption of east rift zone, Kilauea volcano, Hawail (abs.): EOS, Transactions of the American Geophysical Union, v. 65, p. 1130.

Wolfe, E.W., 1984, The volcanic landscape of the San Francisco volcanic field: in Smiley, T.L., Nations, J.D., Pewe, T.L., and Schafer, J.P. (eds.), Landscapes of Arizona -- The Geological Story, University Press of America, Washington, D.C., p. 111-136.

Wolfe, E.W., and Light, T.D., 1984, Strawberry Crater Roadless Areas, Arizona: in Marsh, S.P., Kropschot, S.F., and Dickinson, R.G., editors, Wilderness Mineral Potential - assessment of mineral-resource potential in U.S. Forest Service lands studied 1964-1984: U.S. Geological Survey Professional Paper 1300, vol. 1, p. 110-112.

Wolfe, E.W., and McColly, R.A., 1984, Arnold Mesa Roadless Area, Arizona: 1n Marsh, S.P., Kropschot, S.J., and Dickinson, R.G., editors, Wilderness Mineral Potential - assessment of mineral-resource potential in U.S. Forest Service lands studied 1964-1984: U.S. Geological Survey Professional Paper 1300, vo1. 1, p. 48-51.

Wolfe, E.W., Ulrich, G.E., Holm, R.F., Moore, R.B., and Newhall, C.G., in press, Geologic map of the central part of the San Francisco volcanic field, Arizona: U.S. Geological Survey Miscellaneous Investigations Map I- , 1:50,000. Branch Chief app. (Central Regional Geology) October 5, 1984. (Comprehensive descriptive text, $69 \mathrm{~ms}$ pages plus tables.)

Worner, G., and Wright, T.L., 1984, Evidence for magma mixing within the Leacher See magma chamber (East Eifel, Germany): Journal of Volcanology and Geothermal Research, v. 22, p. 301-328.

Wright, T.L., 1984, Origin of Hawalian thole1ite: a metasomatic model: Journal of Geophysical Research, v. 89, p.3233-3252. 